\title{
ADAPTIVE COMPONENT USAGE FOR THE THERMAL MANAGEMENT OF PICOSATELLITES
}

\author{
A Thesis \\ presented to \\ the Faculty of California Polytechnic State University, \\ San Luis Obispo
}

In Partial Fulfillment

of the Requirements for the Degree

Master of Science in Aerospace Engineering

by

William Dudley Whalen

June 2011 
(C) 2011

William Dudley Whalen

ALL RIGHTS RESERVED 
COMMITTEE MEMBERSHIP

TITLE:

Adaptive Component Usage for the Thermal MANAGEMENT OF PICOSATELLITES

AUTHOR:

William Dudley Whalen

DATE SUBMITTED:

June 2011

COMMITTEE CHAIR: Dr. Jordi Puig-Suari, PhD

COMMITTEE MEMBER: Dr. Kira Abercromby, PhD

COMMITTEE MEMBER: Dr. Kristina Jameson, PhD

COMMITTEE MEMBER: Dr. Robert Crockett, PhD 


\section{ABSTRACT \\ ADAPTIVE COMPONENT USAGE FOR THE THERMAL MANAGEMENT OF PICOSATELLITES}

William Dudley Whalen

The CubeSat standard originated in 1999. It was a joint development led by Dr. Jordi Puig-Suari of California State Polytechnic University San Luis Obispo and Professor Robert Twiggs of Stanford University. The engineering challenges that have come from this picosatellite class have created incredible educational opportunities for engineering students throughout the world. Since the challenges of engineering a CubeSat abound the designers are always looking at novel and even revolutionary solutions to each one. One of those opportunities is in thermal subsystem design, implementation and characterization. A potential solution for CubeSats is adaptive component usage.

This thesis is the written catalogue of my study of adaptive component utilization to solve the thermal management problem inherent in picosatellites. Inside the limited design space of a picosatellite's electrical, mechanical and software subsystems active spacecraft thermal control often is a necessary forfeiture. This does not preclude CubeSat teams from addressing the thermal aspect of spacecraft design. To the contrary it forces them down a different route to ensuring the spacecraft is verified to meet appropriate environmental constraints. Most CubeSat teams, Cal Poly included, use punishing qualification testing, robust system design and a restricted spacecraft operational lifespan ensure their system will operate through all of the environments it will encounter during launch, separation, spacecraft activation and on until the end of operations.

The testing, engineering and modeling I performed were to answer the hypothesis, can a standard ${ }^{\star} 1-U$ CubeSat utilize existing hardware and software to improve its thermal condition and operational lifetime?

This hypothesis assumes thermal control or situational improvement would have to be gained without the addition of thermal control surfaces, active heaters, heat pipes or louvers and no additional flight software. Ground control software and operation alterations were explicitly not included in these assumptions.

The thesis began with defining the many unknowns that existed in the material properties. This required: research into the methods required, specialized measurement hardware to be obtained and set-up, controlled measurements to be taken and thorough testing procedures to be developed. Once the unknowns were better defined the thesis required a detailed satellite thermal analysis by 
multiple methods along with both thermal vacuum chamber simulation trials and finally on-orbit testing.

Based on the research, modeling and testing performed and results obtained through this study, yes, a standard* $1-\cup$ CubeSat utilizing existing hardware and software can improve its thermal condition and operational lifetime. As is shown in Section 3.0 and discussed in detail in Section 4.0, utilizing only the onboard electronics and existing flight software the orbital temperature delta that components are experiencing can be reduced by up to $35.8 \%$. Further analyses in section 4.0 use the temperature data to show that by lowering the temperature deltas the satellite does in fact have the capability to both improve its lifetime and certain key subsystem performance parameters.

Keywords: Thermal, CubeSat, Picosatellite, Heat, Radiation. 


\section{ACKNOWLEDGMENTS}

To my parents, your love and constant support have made me who I am today. Thanks for always supporting me no matter what.

For my family, Emily, thanks for putting up with my timeline, changing diapers while I typed and taking long walks with $O$ to the park and around town to help me get this done, I never could have finished this were it not for your sacrifice and effort. Owen, thanks for understanding when dad wasn't much fun and had to sit at the computer for days on end. I know being two years old and having to be quiet in the house so Dad could study was probably harder than writing this thesis but I appreciate it and love you more than the world itself.

To Dr. P, thank you for the inspiration in the lab and keeping the whole CubeSat and PolySat team on the cutting edge of CubeSats. Thank you for believing in me as my advisor and for believing in the value of rigorous testing (and for buying me enough dewars of liquid nitrogen to put this hypothesis to the test)! Lastly, thanks for your vision and work with Prof. Twiggs, without it I never would have had the incredible opportunity to test and command satellites while still in school.

For Roland, you are a mentor, a friend and a constant source of optimism. CubeSat wouldn't be what it is without you and I never would have learned how to operate the T-VAC were it not for your time and patience.

Dr. A, I know you didn't need any extra work during the weeks before finals but you were willing to sign on anyway. Thank you for making the time to be a part of my committee and helping me make this thesis a good piece of engineering!

To all of my friends in the lab - Justin, Hobbes, Bryan, Parin, Austin, Keith, Lori, Jon, David and Ryan. You have all helped me in one way or another and I thank you for it. I cherish all the memories we made in the ATL over those four years. Anyone who said working on satellites is not fun never worked with our team and obviously did not work on small enough satellites.

Dr. J, thank you for being willing to take time from your own research to help me. Your expertise brought a lot to my committee and without your willingness to jump in I wouldn't have been able to get this finished!

The "Foundation" - Kyle, Spencer, Chris, Jacob, Noe, Derek, Kalia, Armen, Simon and Roland. Thank you for going through all the challenges that came before, for never giving up, for the time and hard work you spent. Thank you for listening to my questions, for helping me with data, for always being there. Nanos gigantium humeris insidentes my friends.

Dr. Crockett, your systems engineering classes were amazing and your passion for the subject helped me become the engineer I am today. Thank you again for agreeing to sign up to my committee. 


\section{TABLE OF CONTENTS}

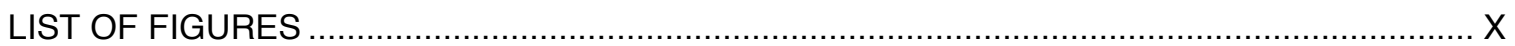

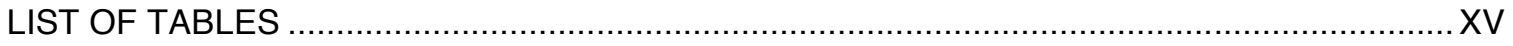

LIST OF COMMON ACRONYMS \& ABBREVIATIONS …................................................II

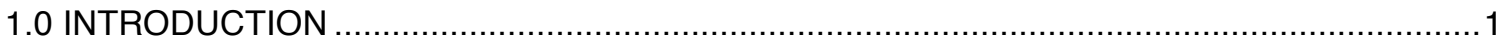

1.1 The History and Background of CubeSats …....................................................

1.2 CubeSat Engineering Challenges and Design Evolutions; Divergence from

Traditional Satellite Analysis and Design ..............................................................

2.0 THESIS MOTIVATION, DRIVERS, GENERAL REASONING AND HYPOTHESIS

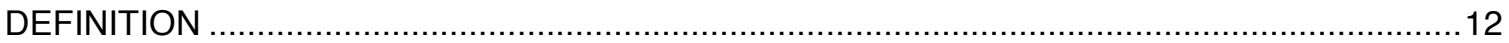

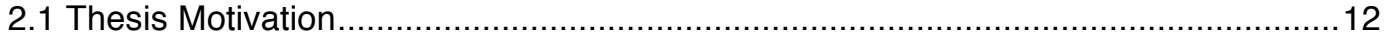

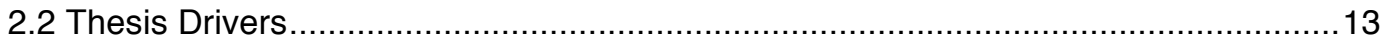

2.3 General Reasoning, Hypothesis Definition, Assumptions and Verification Criteria ....15

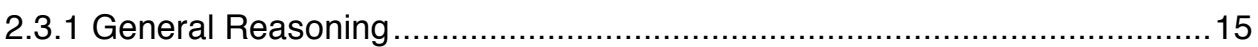

2.3.2 Hypothesis Definition ................................................................. 16

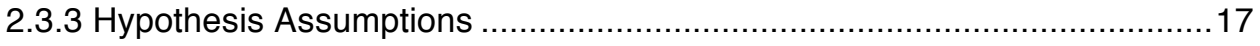

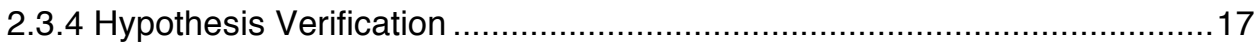

3.0 THESIS BODY; ANALYSES, TESTING \& MODELING REVIEW .....................................2

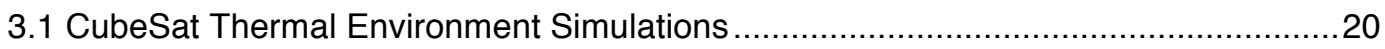

3.2 Defining the Unknowns, Material Properties and Variations ..................................24

3.2.1 6-Layer Flame Retardant \#4 Printed Circuit Boards (PCBs) Radiative

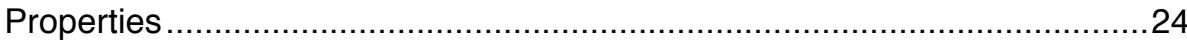

3.2.2 TAIYO PSR-4000BN Liquid Photoimageable Solder Mask Radiative

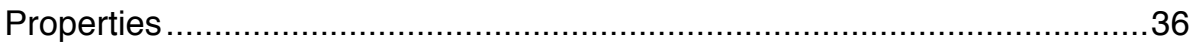

3.2.2.1 A Thermal Vacuum Test Method for Obtaining Emissivity Values ..........37 
3.2.2.2 An Improved Emissivity Test Method Via the RAYTEK MI40

3.2.3 Conformal Coat (IPC A-610 Compliant) Radiative Properties

3.2.4. Improved Triple Junction (ITJ) and Ultra Triple Junction (UTJ) Solar

Cells 55

3.3 Thermal Analyses and Models 57

3.3.1 P's Office 57

3.3.2 Satellite Thermal Analysis Overview 58

3.3.3 General Thermal Equilibrium Calculation (Analytical Baseline) .70

3.3.4 Heat Generation by Mode. .77

3.3.5 Heat Flux Path Mapping via Resistor Model. .87

3.3.6 Finite Element Model Generation and Solutions . .92

3.3.7 Finite Difference Models TRASYS/TSS-SINDA 104

3.4 Active Thermal Vacuum Testing ............................................................... 106

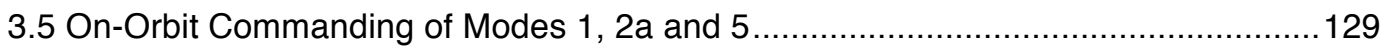

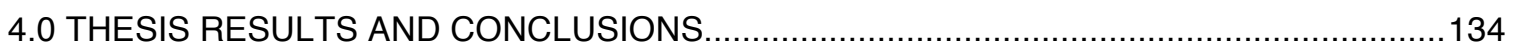

4.1 Review of Results Against Verification Criteria............................................. 134

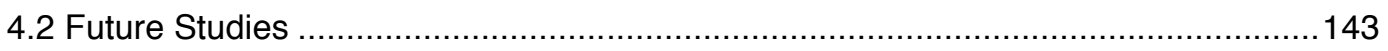

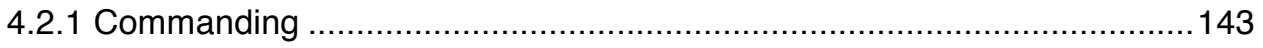

4.2.2 Passive Thermal Control Improvements.............................................145

4.2.3 Improved Capabilities with Increased Power Budgets...........................145

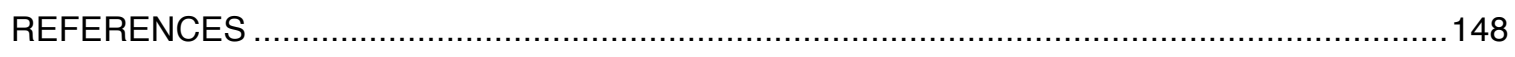

APPENDIX A. CP3 POWER STORAGE THERMAL \& LIFE PROPERTIES ${ }^{28} \ldots \ldots \ldots \ldots \ldots \ldots \ldots \ldots . . . . . . . . . . . . .151$

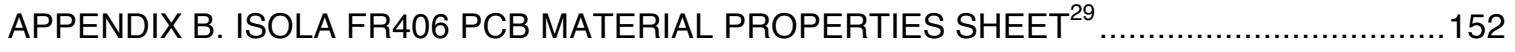

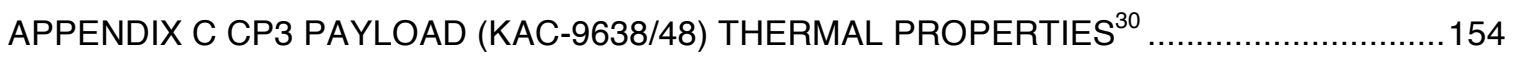

APPENDIX D - POPULATED PCB CALCULATIONS \& ADJUSTMENTS ..............................156 
APPENDIX E. SIERRA PROTO EXPRESS SOLDER MASK PROPERTIES

APPENDIX F. INITIAL CALCULATIONS (HISTORICAL REFERENCE)... 161

APPENDIX G. PROJECTED AREA CALCULATIONS 162

APPENDIX H. THERMAL EQUILIBRIUM WORST-CASE HOT/COLD. 164 APPENDIX I. THERMAL RESISTANCE CALCULATIONS 167 APPENDIX J. TSS AND SINDA MODELING 168 APPENDIX K. SMC THERMAL GRADIENT FAILURE RATES 176

APPENDIX L. RSSI - TEST PROCEDURE \& RESULTS 179 APPENDIX M. COMPONENT TEMPERATURE SENSITIVITIES 190 APPENDIX N. NASA THERMAL CYCLE GUIDELINES PT-TE-140230 196 APPENDIX O. TEMPERATURE MEASUREMENT ON THE CP BUS ${ }^{37}$ 198 APPENDIX P. GENESAT-1 TELEMETRY AND TEMPERATURE RANGES. 202 


\section{LIST OF FIGURES}

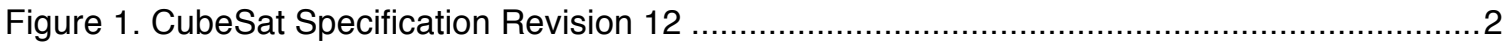

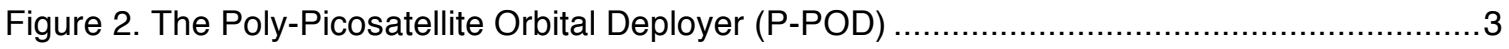

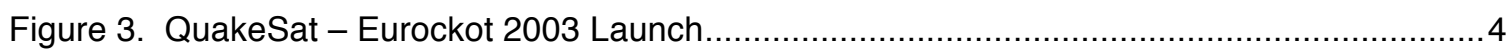

Figure 4. Colony Triple Cube Spacecraft - Falcon 9 Launch ................................................ 4

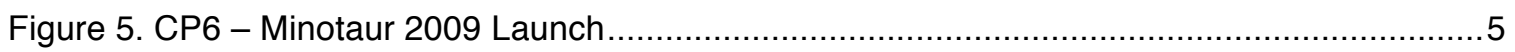

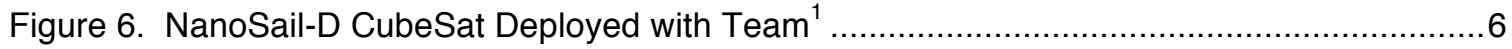

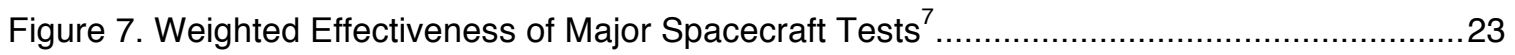

Figure 8. PC104 Standard Board Layout and Mechanical Requirements ${ }^{8}$...............................25

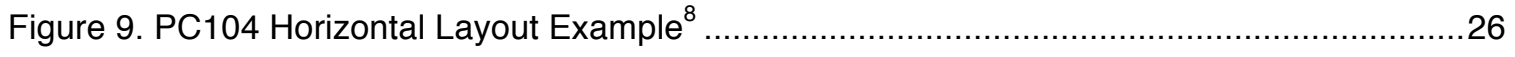

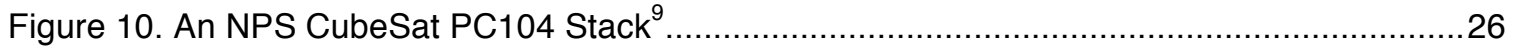

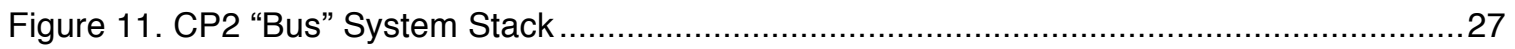

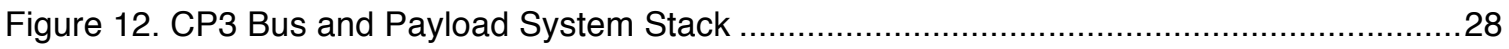

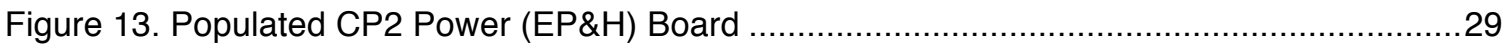

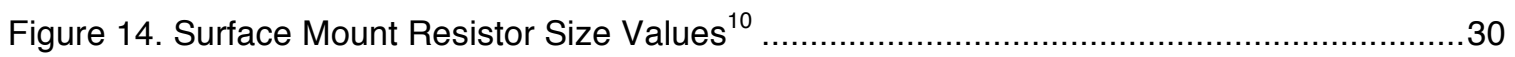

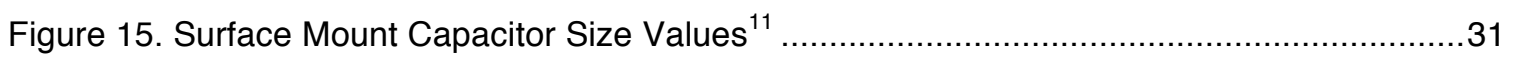

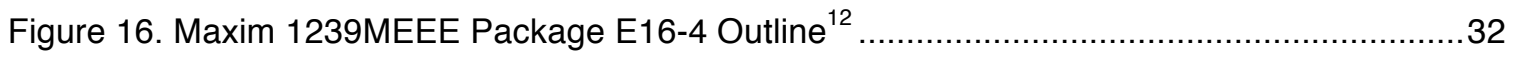

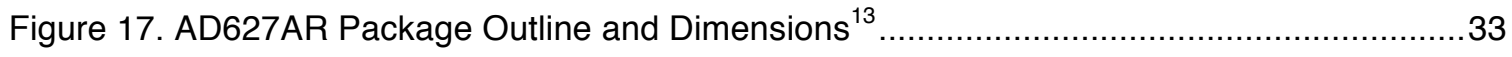

Figure 18. Emissivity/Absorptivity TVAC Test with Thermistor Locations ....................................38

Figure 19. Teflon Thermal Standoff Illustration Pre-Shroud \& with Shroud ................................41

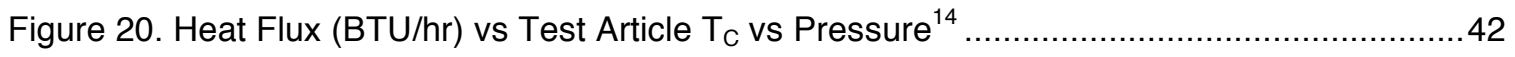

Figure 21. Emissivity Reference Panel from MI40 Operator's Guide …...................................47

Figure 22. Raytek Thermalert Ml40 w/ Breakouts Wires Installed ........................................47

Figure 23. Raytek Thermalert MI40 Post Emissivity Adjustment............................................ 48

Figure 24. IR Sensor Protective Cover and Cover Removed for Testing ................................. 49

Figure 25. Payload Face Board used for Testing (pre-3M Taping) .........................................50

Figure 26. Payload Face Board used for Testing (post-3M Taping) .........................................50 
Figure 27. Sensor "Spot Diameter" versus Distance from Raytek Operators Guide.

Figure 28. Solder Mask Emissivity Test Comparison ....

Figure 29. Spectrolab ITJ and UTJ Cell Radiative Values ${ }^{16}$ .55

Figure 30. Incident Radiation on a Surface ${ }^{2}$ .59

Figure 31. Corrected Satellite Surface Property Averages (Satellite Whole). 62

Figure 32. Solar Albedo Calculation Chart ${ }^{2}$ 65

Figure 33. Earthshine IR Calculation Chart ${ }^{2}$...... 67

Figure 34. Earthshine IR for a LEO Spacecraft (Sample) $)^{2}$ 68

Figure 35. Combined On-Orbit Heat Loads ${ }^{2}$. .68

Figure 36. Worst Case Cold Condition ..... .69

Figure 37. Worst Case Hot Condition 69

Figure 38. Satellite Area Projection Calculator - Surface Area vs. Attitude 71

Figure 39. External On-Orbit Temperature Maximums (Externals) …..................................... 72

Figure 40. External On-Orbit Temperature Maximums (Externals) …...................................... 74

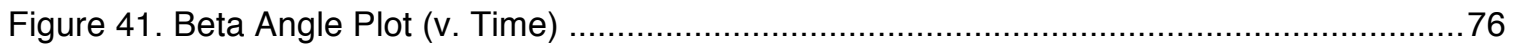

Figure 42. CP3 Lighting Conditions (12 Hour Plot) ...................................................... 77

Figure 43. Baseline Three Hour Post-Launch Run (Detail View) ............................................ 82

Figure 44. CP3 with 1.062W "Standard Operations" Internal Heat Dissipation ...........................83

Figure 45. Mode 2b. Increased 60s Beacon Rate with 1.78W Internal Heat Dissipation .............84

Figure 46. Mode 5 - Increased Data Drop Simulation with 3.38 W Internal Heat Dissipation.......84

Figure 47. Mode 4a. ADC System "ON" with 5.09 W Internal Heat Dissipation ........................... 85

Figure 48. Mode 4c. ADC System "ON" and 60s Beacon Rate with 5.74 W Internal Heat Dissipation. .85

Figure 49. Heating Case with 9 Watt Internal Heat Dissipation............................................. 86

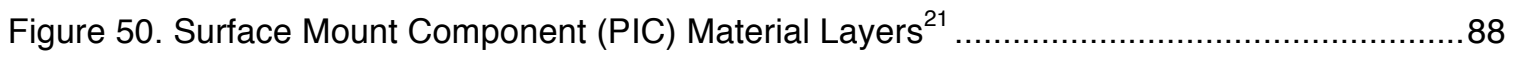

Figure 51. Surface Mount Component Resistance Network \& Heat Flow (Example) ${ }^{21} \ldots \ldots \ldots \ldots \ldots . . . . .88$

Figure 52. Resistance Model for CubeSat Side-Panel with Example Layout ..............................89 


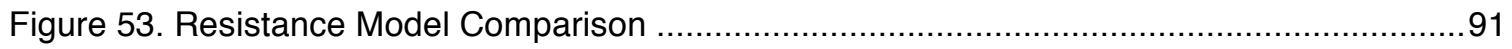

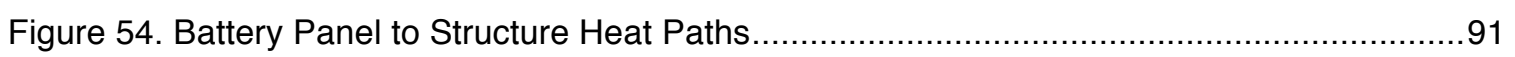

Figure 55. CP3 Internals Exposed - SolidWorks Model w/o C\&DH Board and Side-panels ........95

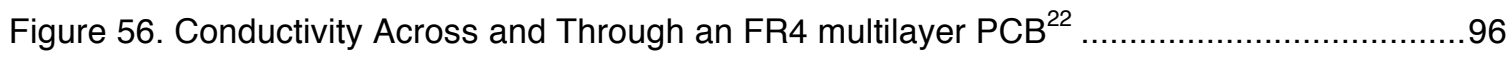

Figure 57. 6-Layer Printed Circuit Board Design \& Ground Plane Illustration ${ }^{23}$...........................97



Figure 59. NPS CubeSat Payload PCB Board Showing Layers and Traces ${ }^{9}$.............................99

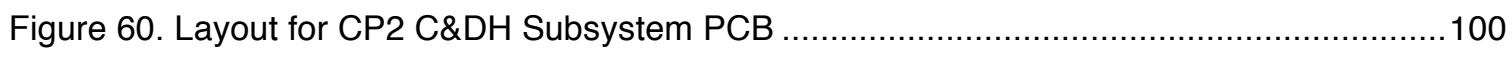

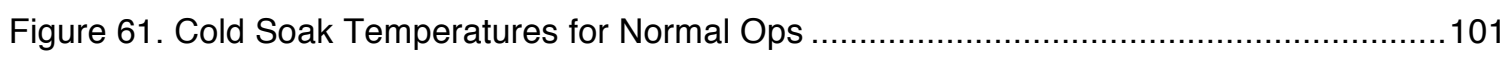

Figure 62. Cold Soak Temperatures Operating in Mode 2a .............................................. 102

Figure 63. Solar Panel Mesh Pre-Analysis ................................................................. 103

Figure 64. De-Integrated Satellite Prior to Thermistor Installation .......................................... 107

Figure 65. Thermistor Bundle Integrated Prior to Testing................................................. 108

Figure 66. T-VAC PC with Labview, Test Plan \& Radio .................................................... 109

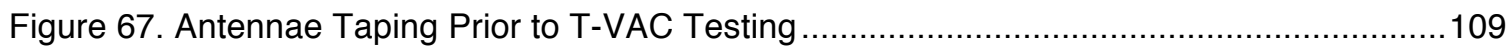

Figure 68. Preparing the Satellite for Testing; Thermistor Bundle \& Antennae Release .............110

Figure 69. Marconi and Hertz, PolySat Groundstation Computers, Antennae Controllers and

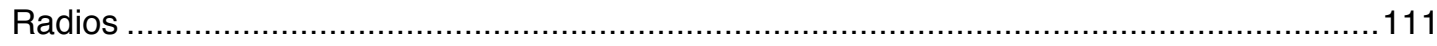

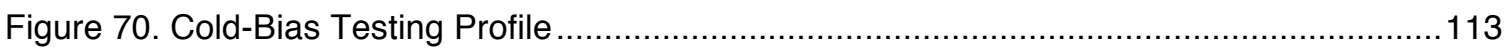

Figure 71. Expected Environment (Thermal Shroud) Temperature with Inertia Effects ..............115

Figure 72. Standard Operations "Mode 1" Cycle Temperatures...........................................117

Figure 73. Omega Controller with National Instruments DAQ ............................................118

Figure 74 Solenoid Valve \& Liquid Nitrogen Dewar during Testing ....................................118

Figure 75. Post Test Inspection \& Antennae Tape-Down .................................................. 120

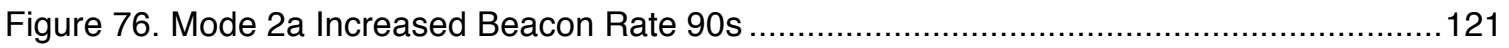

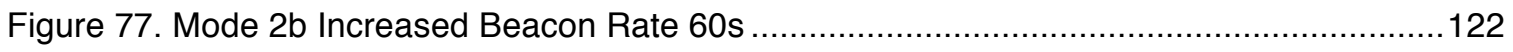

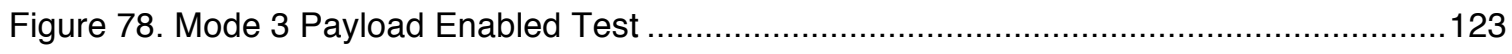


Figure 79. Combined Mode 4a, 4b and Mode 1 Insert Test

Figure 80. Mode 5 Increased Data Drop (Contact) Test.

Figure 81. External On-Orbit Temperatures 1, CP3 130

Figure 82. External On-Orbit Temperatures 2, CP-3 130

Figure 83. Initial On-Orbit Temperatures External 1. 131

Figure 84. Initial On-Orbit Temperatures External 2...... 131

Figure 85. CP2 vs CP3 On-Orbit Temperature Comparison 133

Figure 86. Payload Current Draw Across Temperature Spectrum ${ }^{25}$. 141

Figure 87. Spacecraft ops availability before and after GENSO ${ }^{26}$ 143

Figure 88. GENSO Network coverage for LEO Satellites ${ }^{27}$ 144

Figure 89. Kapton Enclosed Foil Strip Heater 146

Figure 90. Panasonic Prismatic Lithium Ion Operating Characteristics. 151

Figure 91. FR406 Material Properties Sheet 152

Figure 92. FR406 Material Properties Sheet (cont'd) ..... 153

Figure 93. KAC-9638 Monochromatic CMOS Operating Temperature Limits. 154

Figure 94. KAC-9648 Color CMOS Operating Temperature Limits. 155

Figure 95. Sierra Proto Express Tech Specialist Reference Letter 160

Figure 96. Initial Proof-of-Concept Calculation with Assumptions 161

Figure 97 Parallel Flat Plates View Factor (Configuration Factor) ${ }^{31}$ 167

Figure 98. TSS Orbital Input Window and Heating Propagation 169

Figure 99. TSS Variables Input. 169

Figure 100. TSS Variables Input Cont'd 170

Figure 101. SINDA Output - 24 Hour Nominal (Standard Operations) 170

Figure 102. SINDA Orbital Heating for One Orbit.... 171

Figure 103. SINDA Output Mode 1 Standard Operations 171

Figure 104. SINDA Output Mode 2a Increased Beacon Rate 172

Figure 105. SINDA Output w/ High Beacon Rate \& High Sensor Snap Rate (Schema 2b) 172 
Figure 106. SINDA Output w/ Payload Enabled (Schema 3)

Figure 107. SINDA Output w/ ADC On (Schema 4a)

Figure 108. SINDA Output w/ ADC On \& 90s (Schema 4b) 174

Figure 109. SINDA Output w/ ADC On \& 60s (Schema 4c) 174

Figure 110. SINDA Output w/ Increased Contact/ Data Drop (Schema 5) ..... 175

Figure 111. PCB Article for Surface Mount Component Failure Rate Test ${ }^{32}$ 176

Figure 112. Solder Joint Cracking due to Thermal Cycling \& Alteration in Resistance ${ }^{36}$ 177

Figure 113. 1000 Cycle Thermal Test on 3216 Surface Mount Resistor...... 177

Figure 114. Failure by Resistor Type and Cycle Number for Different Ramp Gradients ${ }^{32}$ 178

Figure 115. RSSI Receive Sensitivity vs. Power Inputs (Use for Comparison) 187

Figure 116. C\&DH Temperature During Cycle \#1 188

Figure 117. MAG Data Readings During Cycle \#1 ... 188

Figure 118. RSSI Values For Cycle \#1 189

Figure 119. Citizen Crystal Oscillator Variances ${ }^{33}$...... 190

Figure 120. CC1000 and C\&DH Reference Oscillator - Citizen HCM49 ${ }^{33}$..... 191

Figure 121 XO (Uncompensated) Crystal Oscillator Temperature Shift 192

Figure 122. Supply Current vs. Temperature for the DS323 $1^{34}$ 192

Figure 123. Frequency Deviation vs. Temperature and Aging Value for the DS3231 ${ }^{34}$ 193

Figure 124. Delta Time and Frequency vs. Temperature for the DS3231 ${ }^{34}$......... 194

Figure 125. Shutdown Supply Current vs. Temperature for MAXIM 1239 ADCs ${ }^{12}$ 194

Figure 126. Lithium Ion Electrolyte Conductivity Over Temperature ${ }^{35}$ 194

Figure 127. Specific Energy versus Temperature by Battery Type ${ }^{35}$ 195

Figure 128. Lithium Ion Anode Degradation ${ }^{36}$ 195

Figure 129. Resistance to Temperature for a NHQM Series Thermistor by Thermometrics. ${ }^{9} \ldots 199$

Figure 130. ADC Code vs. Thermistor Temperature. 199

Figure 131. GeneSat-1 Temperature History (External) ${ }^{38}$..... 202

Figure 132. GeneSat-1 Beacon Data Including Thermal Ranges ${ }^{38}$ 203 


\section{LIST OF TABLES}

Table 1. Size Differences (By Mass); CubeSats vs Industry Satellites.......................................

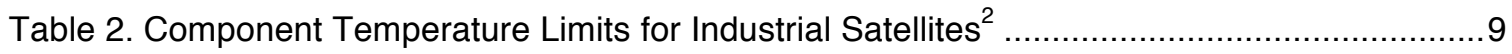

Table 3. Component Temp. Limits from CubeSat Parts vs. Industry.........................................10

Table 4. Populated PCB Area and E/Alpha Adjustments - Side Panel Interior............................34

Table 5. Populated PCB Area and E/Alpha Adjustments - Side Panel Exterior (Solar Panels)....34

Table 6. Populated PCB Area and E/Alpha Adjustments -C\&DH Board (Satellite Interior) ...........35

Table 7. Emissivity Obtained Through Thermal Vacuum Testing Method ................................43

Table 8. Solder Mask Emissivity Test Results................................................................ 52

Table 9. Emissivity Values for Conformal Coated Parts .................................................................54

Table 10. Standard Spacecraft Emissivity, Absorptivity and Alpha/Epsilon Values ${ }^{19}{ }^{1 . . . . . . . . . . . . . . . . .61 ~}$



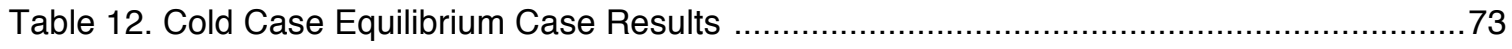

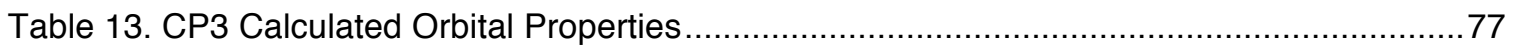

Table 14. Heat Generation by Component/System (CP3) .................................................... 78

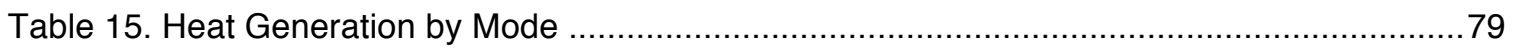

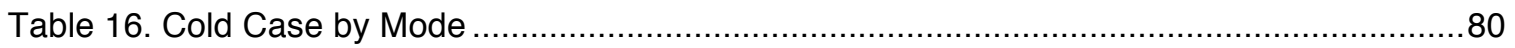

Table 17. Criteria 1.1 Verification Evidence; Percent Reduction Maximum to Minimum ..............81

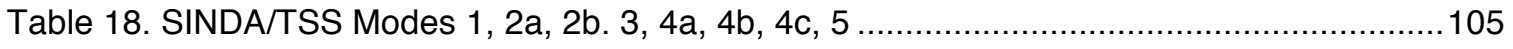

Table 19. Thermal Vacuum Chamber Test Results .........................................................128

Table 20. Verification Criteria Compilation …….......................................................... 134

Table 21. Criteria 1.1 Verification Evidence; Percent Reduction Max to Min ............................135

Table 22. SINDA Output Results Summary by Mode .........................................................135

Table 23. Criteria 1.1 Thermal Vacuum Chamber Test Results ...........................................136

Table 24. Surface Area by Representative Component \& Property Adjustment ....................... 156

Table 25. Side Panel Interior Emissivity Calculation (Correction) ...........................................157

Table 26. Side Panel Exterior Absorptivity and Emissivity Calculation (Correction) ...................157 
Table 27. C\&DH Board Emissivity Calculation (Correction)

Table 28. Standard Side Panel Corrected Emissivity and Absorptivity Values 158

Table 29. $+Z$ \& $-Z$ Side Panel Corrected Emissivity and Absorptivity Values..... 158

Table 30. Antenna Side Panel Corrected Emissivity and Absorptivity Values 159

Table 31. Corrected Satellite Surface Averages (Satellite Whole) 159

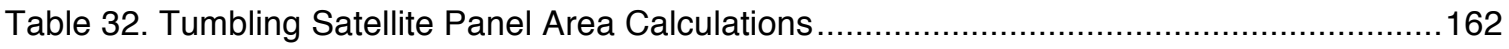

Table 33. Tumbling Satellite Panel Area Calculations (cont'd) ......................................... 163

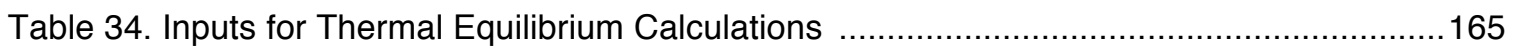

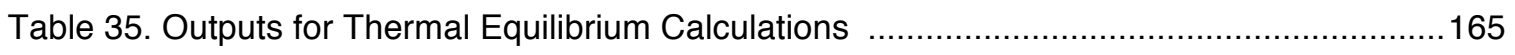

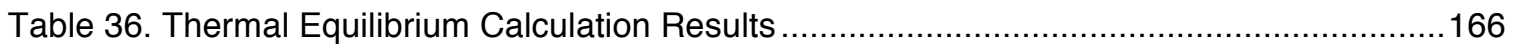

Table 37. Adjusted "Worst-Case" Hot w/ Adjusted Internal Heat Flux ................................. 166 


\section{LIST OF COMMON ACRONYMS \& ABBREVIATIONS}

$\mathrm{C \& DH}$

CONOPS

COTS

CAL POLY

$\mathrm{CP}$

EP\&H

GEVS

LEO

NASA

PCB

P-POD

TVAC
Command and Data Handling

Concept of Operations

Commercial-Off-the-Shelf

California Polytechnic State University

Cal Poly

Electrical Power and Handling

General Environmental Verification Specification

Low Earth Orbit

National Aeronautics and Space Administration

Printed Circuit Board

Poly-Picosatellite Orbital Deployer

Thermal Vacuum Chamber 


\subsection{Introduction}

\subsection{The History and Background of CubeSats}

When Dr. Jordi Puig-Suari and Dr. Robert Twiggs came up with the CubeSat standard both agreed that a small, common satellite standard could be an enabler. It could provide students and small companies worldwide the ability to design, test and launch a flight quality satellite. This experience was previously inaccessible for the vast majority of university students and the capability was originally beyond most small businesses. The true embodiment of the "learn by doing" mantra, the CubeSat project helped to fill this crucial niche. Below are some of the benefits it provided.

- Giving students the real-world satellite design, integration and launch experience.

- Providing companies the ability to test new components on-orbit with low cost and risk.

- Giving the satellite industry a development path to new concepts and innovative designs.

The CubeSat Specification Revision12 front page (figure 1) allows for a cube $100 \mathrm{~mm}$ on each side and with a mass of $1330 \mathrm{~g}$ or less. This standard is the newest revision currently published by the California Polytechnic State University CubeSat project. 


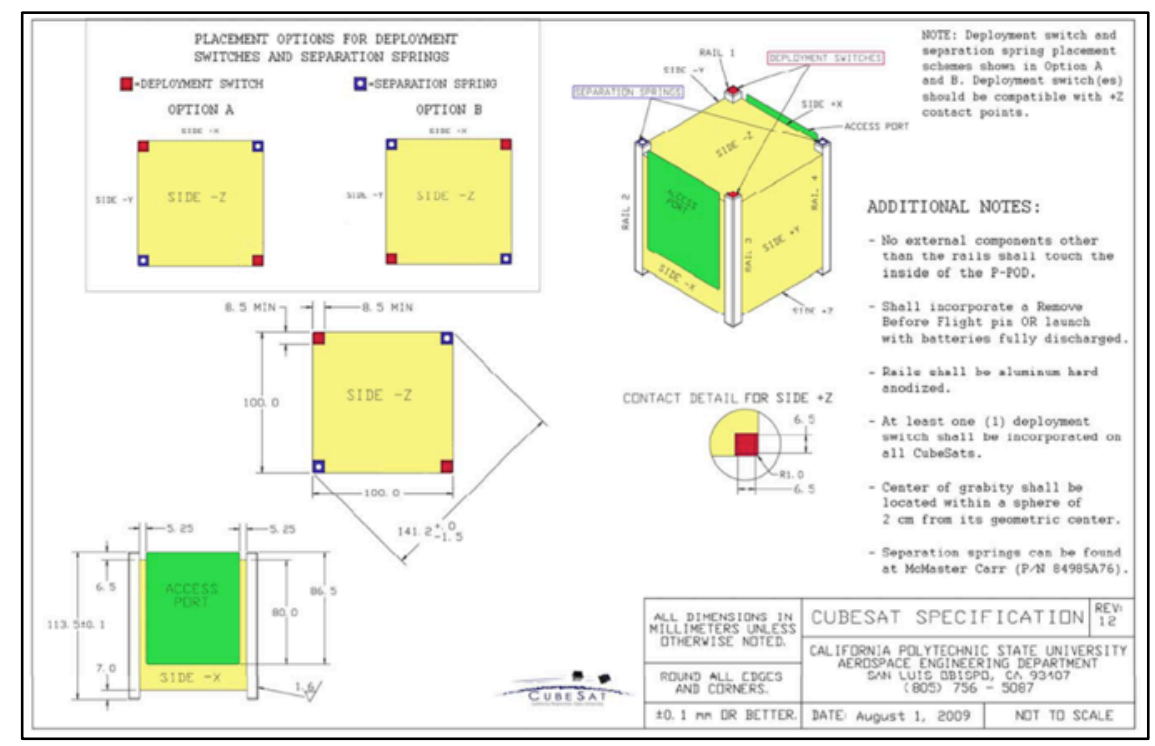

Figure 1. CubeSat Specification Revision 12

Alternative CubeSat designs, per the standard, can also utilize a $200 \mathrm{~mm}$ or $300 \mathrm{~mm}$ length and a $100 \times 100 \mathrm{~mm}$ cross-section with an approximate 2 kilogram or 4 kilogram mass limitation, respectively. Along with this satellite standard, the P-Pod or Poly-Picosatellite Orbital Deployer, shown in figure 2, has enabled CubeSats to be simply and reliably integrated onto nearly all existing space launch vehicles (LVs) at very low cost and with no impact on the primary LV payload. 


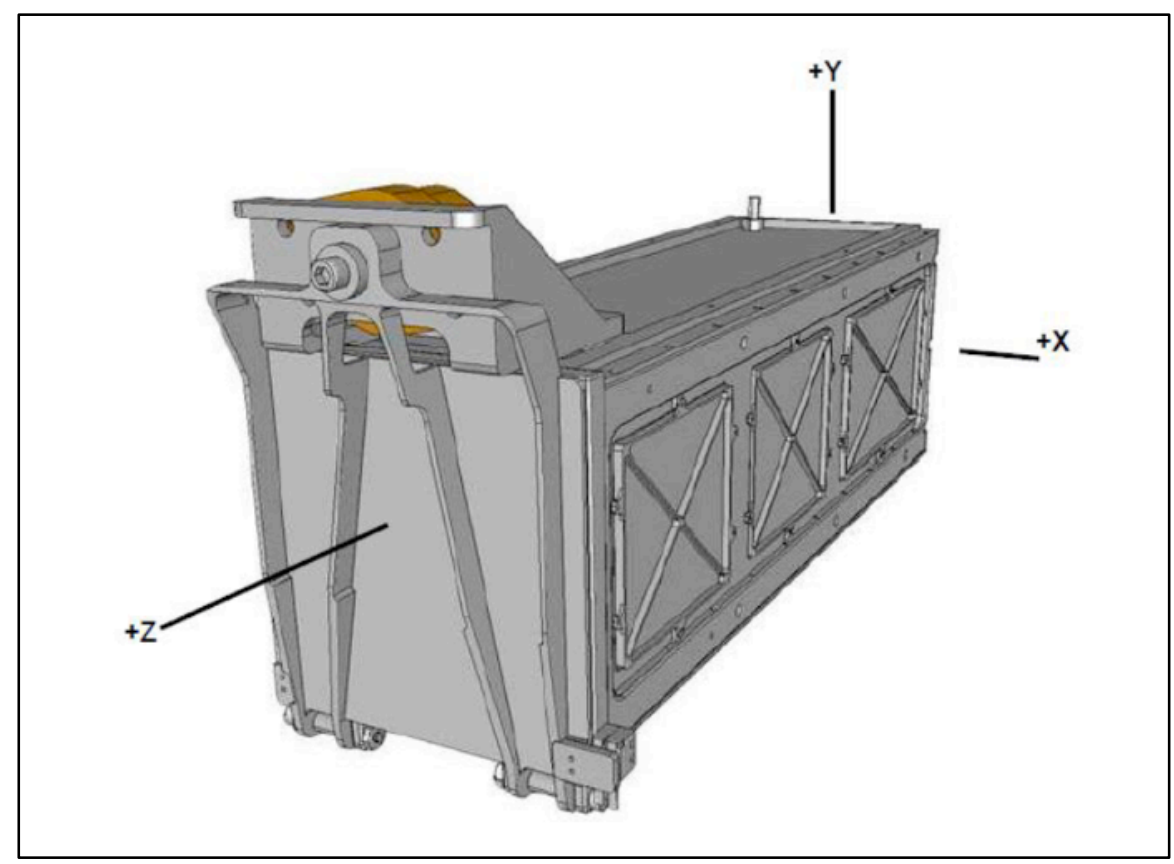

Figure 2. The Poly-Picosatellite Orbital Deployer (P-POD)

Launch providers from Russia and India to the United States have begun offering ride-sharing opportunities that utilize the P-Pod. Thanks to standardization in the satellite form factor and reliability in the integration mechanism increasing numbers of CubeSat class payloads are being launched. Launches began in 2003 with the Eurockot launch of six CubeSat class payloads including the first ever triple cube shown in figure 3, QuakeSat. 


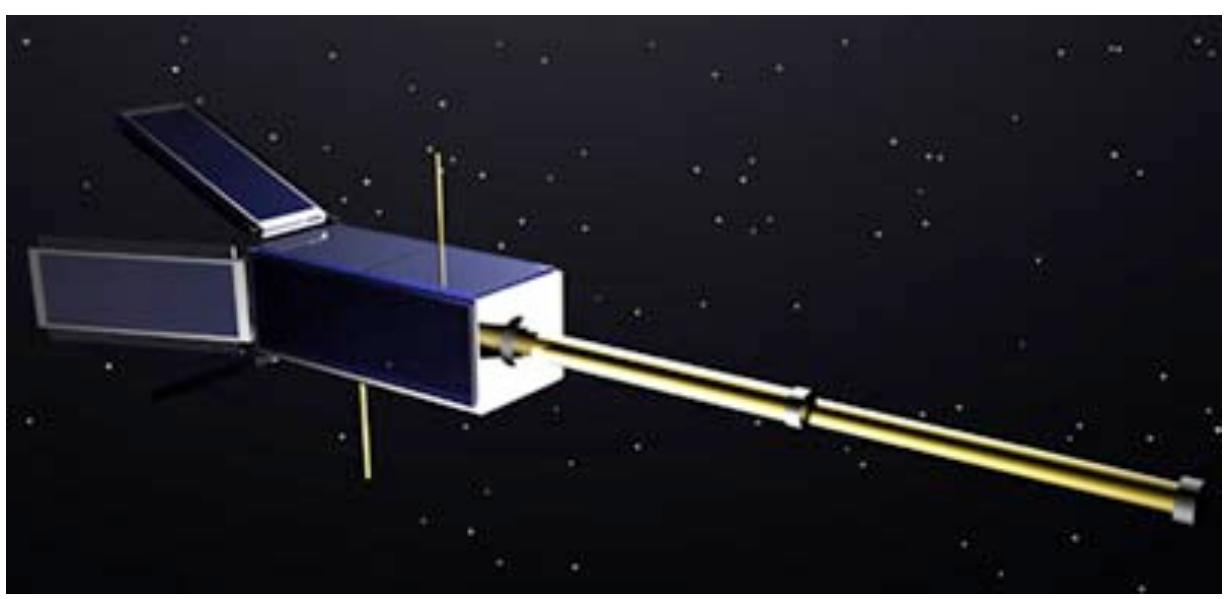

Figure 3. QuakeSat - Eurockot 2003 Launch

CubeSat launches continue today with the recent SpaceX Falcon 9 launch that lofted several triple cubes on December $8^{\text {th }}$ 2010. In figure 4 is a sample of the triple cube Colony spacecraft launched on the SpaceX Falcon 9.



Figure 4. Colony Triple Cube Spacecraft - Falcon 9 Launch

Thanks to inexpensive launch costs, community outreach and regular conferences over the past ten years the CubeSat development community itself has matured from only a handful of schools in Europe and the United States to 
several hundred developers worldwide. As an example of the evolution more recent CubeSats launched, CP6, is shown below in figure 5. This satellite was originally built as the back-up flight model for CP3 launched on the second DNEPR mission. Since it was never used, the CP3 back-up was modified, a new payload was added along with a low noise amplifier (LNA), and it was launched with two other 1-U CubeSats out of Wallops, VA.

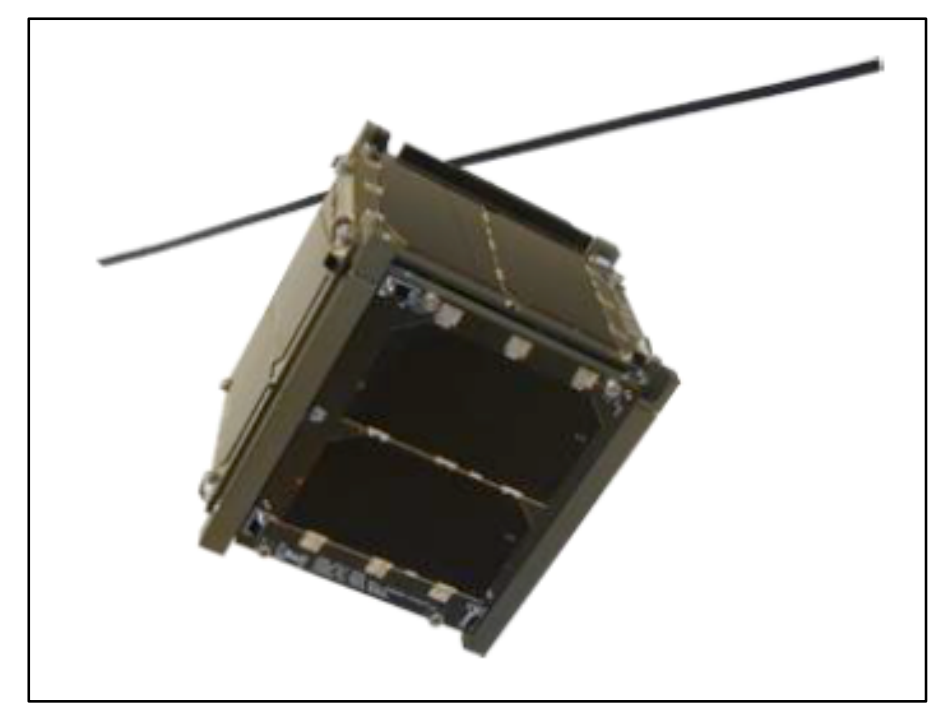

Figure 5. CP6 - Minotaur 2009 Launch

The mission and requirements driving the design of a CubeSat can vary widely. This is another challenge faced by the picosatellite thermal engineer. The specification really is flexible enough that almost any payload that can fit in the satellite can be flown. Payloads that have flown include everything from photomultiplier tubes to micro-vacuum thrusters and even the first fully deployed solar sail, NanoSail-D. This wide range of payloads between figure 5 CP6 and figure 6 NanoSail-D can create lots of challenges! 


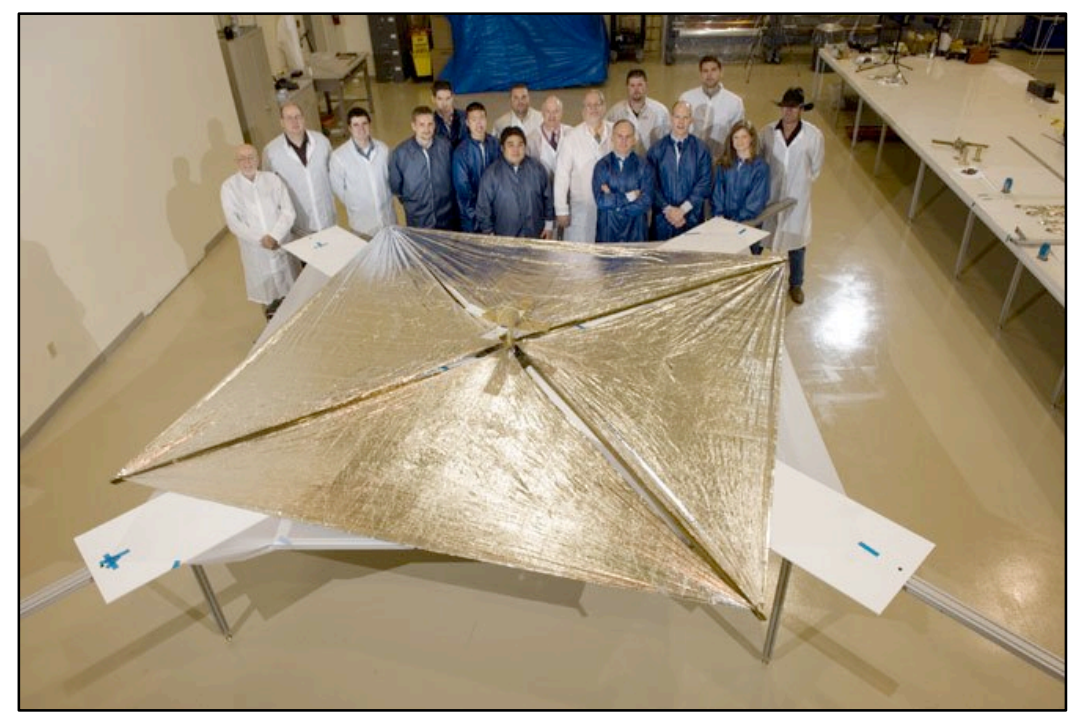

Figure 6. NanoSail-D CubeSat Deployed with Team ${ }^{1}$

CubeSats, like all satellites, perform better and last longer as a system when the components inside them remain within the temperature limits they are designed for. Satellite thermal design and control begins with establishing the temperature specifications within which the satellite will operate during its lifetime. The design of the thermal subsystem must be done such that the established specifications are not exceeded during the satellites orbit. However, to ensure robustness, the satellite must also be designed to have the ability to perform satisfactorily even if subjected to short-term out of specification temperatures. The keys to this challenge are the same as in any engineering challenge, analysis, similarity, tests and innovative design. The next section reviews some of the differences in these keys when it comes to picosatellites. 


\subsection{CubeSat Engineering Challenges and Design Evolutions; Divergence}

\section{from Traditional Satellite Analysis and Design}

With picosatellites the typical models do not always apply. To illustrate the vast technical and analytical differences that exist between the design of picosatellites and their larger cousins consider the following points. The traditional and statistical median satellite is anywhere from several hundred to several thousand kilograms, with the mean being much closer to the latter than the former. This 'average' satellite has an energy consumption of several kilowatts of electrical power along with the production, distribution and storage systems capable of generating this power while on orbit. Accompanying the several metric tons is a surface area that measures in tens of meters squared and a volume that is also exponentially greater than that of a picosatellite. Table 1 was constructed to illustrate the difference that exists between CubeSats and industrial satellites just in the system mass quantity alone.

Table 1. Size Differences (By Mass); CubeSats vs Industry Satellites

\begin{tabular}{|l|r|l|}
\hline & Mass $(\mathrm{kg})$ & Manufacturer \\
\cline { 2 - 3 } SBIRS GEO & 4500 & Lockheed Martin \\
DSP & 2400 & Northrop Grumman \\
GEO-EYE 1 & 1200 & General Dynamics \\
IKONOS & 800 & Lockheed Martin \\
IRIDIUM & 680 & Motorola \\
Pioneer 1 & 38 & TRW \\
XSS-10 & 28 & Boeing \\
CP2/3 & 1 & PolySat - Cal Poly \\
\hline
\end{tabular}


Larger satellites have advantages in the realm of thermal design and analysis. In analysis they have very high accuracy with regards to what a surface is emitting or absorbing due to their budget and the economy of scale principal. Their solar panels are tightly characterized and fitted GalnP2/GaAs/Ge cells and low absorptivity Kapton tape. Their supporting walls are bounded on the exterior by either specialty multi-layer insulation (MLI) and second surface or first surface mirrors. On the interior their electrical housings are coated in $3 \mathrm{M}$ "Velvet Black" paint to provide the maximum radiative heat dispersion. In addition they have heat pipes built into the supporting walls below along with heater elements and thermostats mounted inside or around them. Their dishes/antennae are covered in military grade thermal coatings, all of which have been exhaustively tested and whose composition and thermal properties are well vetted for their degradation due to ultraviolet radiation and charged particles from the beginning to the end of life (BOL \& EOL).

CubeSats can not make the same generalizations, use expensive military standard paint, employ an MLI design and sewing team or ensure their commercial off the shelf (COTS) subcontractors will provide every last material property detail to them. CubeSats must rely on testing, modeling and extensive categorization in order to fully know their thermal properties, especially their irradiative properties.

In the realm of thermal design larger satellites have another advantage to picosatellites. Components on a picosatellite cannot be thermally isolated from 
one another without extensive work and significant mass losses. Refer to the two tables below (tables 2, 3) for a comparison of the temperature ranges on MILSTD parts and subsystems used in large commercial or government satellites against the temperature limits that the COTS components inside a CubeSat should be held to (excerpted from manufacturer data available in Appendix A, B, C).

Table 2. Component Temperature Limits for Industrial Satellites ${ }^{2}$

\begin{tabular}{|l|c|c|}
\hline \multirow{2}{*}{\multicolumn{1}{c|}{ Component }} & \multicolumn{2}{|c|}{ Typical Temperature Ranges $\left({ }^{\circ} \mathrm{C}\right)$} \\
\cline { 2 - 3 } & Operational & Survival \\
\hline Batteries & 0 to 15 & -10 to 25 \\
Power Box Baseplates & -10 to 50 & -20 to 60 \\
Reaction Wheels & -10 to 40 & -20 to 50 \\
Gyros/IMUs & 0 to 40 & -10 to 50 \\
Star Trackers & 0 to 30 & -10 to 40 \\
C\&DH Box Baseplates & -20 to 60 & -40 to 75 \\
Hydrazine Tanks and Lines & 15 to 40 & 5 to 50 \\
Antenna Gimbals & -40 to 80 & -50 to 90 \\
Antennas & -100 to 100 & -120 to 120 \\
Solar Panels & -150 to 110 & -200 to 130 \\
\hline
\end{tabular}


Table 3. Component Temp. Limits from CubeSat Parts vs. Industry

\begin{tabular}{|c|c|c|c|}
\hline $\begin{array}{l}\text { Actual } \\
\text { CubeSat }\end{array}$ & $\begin{array}{l}\text { Comparable } \\
\text { Industry }\end{array}$ & $\begin{array}{l}\text { CubeSat } \\
\text { Component }\end{array}$ & $\begin{array}{l}\text { Industry } \\
\text { Component }\end{array}$ \\
\hline $\begin{array}{l}\text { Batteries } \\
(\mathrm{CGA})\end{array}$ & $\begin{array}{l}\text { Batteries } \\
\text { (Charging) }\end{array}$ & $\begin{array}{l}-10 \text { to } 60{ }^{\circ} \mathrm{C} \\
0 \text { to } 45{ }^{\circ} \mathrm{C}\end{array}$ & -10 to $25^{\circ} \mathrm{C}$ \\
\hline $\begin{array}{l}\text { FR4 Solar } \\
\text { Panels }\end{array}$ & Solar Panels & -20 to $130{ }^{\circ} \mathrm{C}$ & -200 to $130{ }^{\circ} \mathrm{C}$ \\
\hline $\begin{array}{l}\text { Payload } \\
\text { Cameras }\end{array}$ & Star Trackers & 10 to $50^{\circ} \mathrm{C}$ & -10 to $25^{\circ} \mathrm{C}$ \\
\hline
\end{tabular}

As can be seen from the comparison above, the temperature limits are similar. The key takeaway here is that on large satellite system the solar panels aren't thermally coupled with or re-radiating to the batteries and they are thermally isolated from the star trackers. On a CubeSat the external surfaces are both conducting and re-radiating to the interior parts. Larger satellites will often have an entire active thermal subsystem composed of heaters, radiators and heat pipes that can use special materials, designs and electrical energy to moderate or manage the thermal situation and significantly reduce any temperature instability or variance. So components like polymer-based integrated circuits (ICs), for example, that are designed to operated with full confidence in a $0^{\circ} \mathrm{C}$ to $40^{\circ} \mathrm{C}$ environment can be maintained in that environment, while other components like Gallium Arsenide solar cells which operate best anywhere from $-100^{\circ} \mathrm{C}$ to $20^{\circ} \mathrm{C}$ can be isolated from the ICs and passively 
allowed to fluctuate to the lower temperatures with only thermal coatings and MLI to protect against overheating or other thermal issues. This isolation and thermal specificity is achieved through the methods previously discussed along with conduction isolators. Using materials with low conductivity such as ceramics is one way of isolating two components or subsystems with differing thermal ranges. Another method of thermal isolation is to utilize designs that limit the total heat flux by limiting the cross-sectional area available to aforementioned flux.

Picosatellites are obviously limited by mass and volume, so physically limiting the cross-sectional area or utilizing advanced materials are not always available to the thermal designer. Again, with a CubeSat class satellite the majority of your systems have set thermal couplings that cannot be adjusted without incurring serious cost or schedule impacts. 


\subsection{Thesis Motivation, Drivers, General Reasoning and Hypothesis Definition}

\subsection{Thesis Motivation}

The thermal challenges facing picosatellites as discussed in Section 1.2 are numerous. Before beginning this thesis work a qualitative review of the major factors and their impact on the thesis was in order. The list below was developed to attempt to quantify these major factors motivating the thesis.

- Extreme on orbit temperature gradients

- Due to low thermal inertia, lack of control and LEO orbits with eclipse conditions CubeSats undergo constant thermal cycling causing thermal shock and temperature gradients across subsystems

- Unknown thermal environments

- Secondary spacecraft do not choose the orbit nor control the ascent heating or payload fairing jettison conditions. (CubeSat teams can choose a general LEO orbital regime but it limits their opportunities).

- Spacecraft Attitude or Orientation

- CubeSats often lack the ability to accurately control their attitude and more advanced CubeSats that do have attitude control typically use it solely for payload operations, not for thermal orientation or for providing a "thermal roll." 


\subsection{Thesis Drivers}

The factors listed in section 2.2 were great motivators to begin research in thermal control for picosatellite systems. Given these motivating factors the following list of key drivers, or items which the testing for the thesis itself were derived. These were done to attempt to focus the assumptions and begin the hypothesis definition process.

- Increasing orbital debris regulations

- The Federal Communication Commission (FCC), International Telecommunication Union (ITU), National Aeronautics and Space Administration (NASA), European Space Agency (ESA), International Astronautical Congress (IAC) and Committee on Space Research (COSPAR) have all increased the regulations and restrictions on orbital debris, impacting the CubeSat community immensely. The majority of the new standards read similar to the NASA standard: Limiting orbit lifetime after mission completion to 25 years or maneuvering to a disposal orbit. ${ }^{3}$ This drives CubeSats to extend their useful mission or system lifetime so they can ensure they meet these regulations. Using Commercial Off-The Shelf (COTS) products and trying to extend mission life is an engineering dichotomy. 
- Stringent picosatellite budgets

- The budget for volume, mass, power and schedule are all limited for picosatellites. These tight constraints drive a very narrow trade space and check the potential solutions available to CubeSat engineers. This driver pushed the hypothesis to look for an atypical solution. Atypical solutions however are the norm when it comes to CubeSat. Since the beginning of the standard Prof. Twiggs and Dr. Puig-Suari call for CubeSat engineering students to literally "think outside the box" ${ }^{\prime 4}$ which has driven everything from kilometer long hoy-tethers to picosatellite micro-propulsion systems and Stanley ${ }^{\mathrm{Tm}}$ tape-measure body mounted antennas.

With the motivation developed and the drivers established the next step was to find a reasonable solution that could be tested and evaluated to meet the need. In evaluating the motivators and the drivers, reviewing them with team members and the thesis advisor the hypothesis began taking shape. 


\subsection{General Reasoning, Hypothesis Definition, Assumptions and Verification Criteria}

\subsubsection{General Reasoning}

The general solutions proposed to meet the motivation and drivers in Section 2.2, from a thermal perspective, are shown in the list below.

- Modifications to flight hardware and software

- Strict enforcement of testing and qualification programs

- Adaptive component usage and command schema alterations

Since the second generation of Cal Poly satellites built were already integrated into Poly Picosatellite Orbital Deployers (P-PODs) their design and flight hardware were fixed and could not be modified for thermal concerns. Aside from already being integrated the increased cost, time, mass, power and effort required to implement this option is out of scope for most CubeSat teams (Cal Poly included).

The second option was that which the Cal Poly series of satellite actually pursued prior to launch and operations. In lieu of adding heater components, controllers, multi-layer insulation (MLI), applying thermal treatments or installing thermal mass the Cal Poly team decided to take an alternate path of aggressive testing and qualification. This testing is reviewed and discussed in greater detail later in this thesis (section 3.0). 
The last option considered was a new concept for picosatellites and as such it had the required originality suited to a masters level thesis. There was opportunity to define, model and test this option without making flight system alterations. It also would require critical and independent thinking with a substantial undertaking to provide the evidence, combine evidence types and analyze them for conclusions and verification of the hypothesis. For the aforementioned reasons, the third solution was chosen as the basis for the thesis.

\subsubsection{Hypothesis Definition}

Using the third solution discussed in section 2.3 the following hypothesis was made:

Can a standard 1-U CubeSat utilize existing hardware and software to improve its thermal condition and operational lifetime?

My stance and thesis are that based on first order calculations, material properties testing, fundamental engineering analyses, Finite Element Analyses (FEAs), Finite Difference models (FDs), thermal vacuum environment simulations and finally the on-orbit testing discussed in section 3 and section 4 , yes, a 1-U CubeSat through adaptive component usage, is able to improve its thermal condition and operational lifetime with its existing hardware and software. However before that thesis could be posed and backed with data, the hypothesis 
needed simplifying assumptions, modeling constraints and clear verification criteria to justify this stance and thesis to be truth.

\subsubsection{Hypothesis Assumptions}

To appropriately bound the study, ensure accurate results and have precision between verification methods (test, direct analysis, FEA/FD) the following assumptions were made.

- No additional mass is available to provide for either thermal control or alter the systems' thermal inertia.

- No changes are to be made to the system volume, geometry or surface areas.

- No flight software modifications can be made.

- Existing steady-state on orbit temperatures are already within the satellite's component survival levels (CSLs).

\subsubsection{Hypothesis Verification}

To verify that the hypothesis had been met and the thesis proved, clear and concise success criteria were established prior to testing and modeling. The criteria, their implementation or measure and any additional detailed examples are displayed in the format below.

1. Criteria

1.1. Implementation or Measure

1.1.1. Detailed example (if required) 
The list below covers criteria generated to provide clear success or failure during the verification process of the thesis.

1. The thermal condition of the satellite shall be more stable* with the implementation of the thesis than via passive control and standard on-orbit operations. Stability ${ }^{*}$ is defined as achieving one of the following.

1.1. *A $15 \%$ reduction in spacecraft subsystem component temperature variability across maximum and minimum orbital heat flux conditions.

1.2. *A $10 \%$ reduction of in-plane thermal gradients on the subsystem printed circuit boards (PCBs) and an associated (equivalent) reduction in heat-flux across the subsystem PCBs.

2. The satellite shall have improved operations or lifetime via one or more of the following conditions through the implementation of the thesis.

2.1. Increased on-orbit component lifespans (potential, via analysis).

2.2. Improved RF or Electrical Power and Handling (EP\&H) subsystem performance (stability) and/or reliability.

2.2.1. Via measurably higher Received Signal Strength Indicator (RSSI) for the RF performance parameter.

2.2.2. Via higher percentage of acknowledge (ACK) responses during operations for the RF reliability parameter.

2.3. Improved Command and Data Handling (C\&DH) or Inter-Integrated Circuit $I^{2} C$ reliability.

2.3.1. Via lower $\mathrm{CDH}$ Reset Value. 
2.3.2. Via improved $\mathrm{CC} 1000$ or equivalent crystal oscillator operations reliability and lowered drift (Analysis).

2.4. Enhanced payload performance (Realized or Potential).

2.4.1. Via testing performed to support K. Glassey on payload sensitivity and thermal performance.

2.4.2. Via analysis of quantifiable improvements to payload subsystems.

3. Primary or bus system operations must not be negatively impacted by the implementation of the command schema.

3.1. EP\&H subsystem values (BATTA and BATTB) must not have overcurrent or undercurrent faults.

3.2. EP\&H subsystem Depth of Discharge (DoD) must not exceed recommended manufacturer limits. 


\subsection{Thesis Body; Analyses, Testing \& Modeling Review}

\subsection{CubeSat Thermal Environment Simulations}

Before delving into the analysis, testing \& modeling done for this thesis it is critical to discuss what existing CubeSat teams have done in the past to solve the thermal environment challenge. Currently the in order to qualify designs for thermal environments on orbit the CP PolySat and CubeSat team put their satellites through a punishing series of thermal cycle, soak and shock testing that, in leui of having detailed thermal designs, subsystems or entirely MIL-STD qualified and accordingly priced parts certifies that the satellite will indeed operate through the worst scenarios on orbit. (Note some CubeSats do indeed use MIL-STD certified parts.)

By simulating conditions above and beyond the NASA General Environmental Verification Specification (GEVS) the Cal Poly team and other CubeSat developers ensure that their systems are robust enough to withstand the on-orbit environments. It must be noted that this testing is a critical part of the design and testing of any spacecraft. This testing ensures that issues and anomalies can be uncovered while the design is still capable of being altered or modified. Using a thermal vacuum chamber or "T-VAC" the teams simulate the radiative scenarios in space by first creating a vacuum of $10^{-5}$ Torr and then using cooled, typically via liquid nitrogen, or heated, typically via resistive heaters or infrared lamps, walls. This closely imitates the on-orbit environment that the satellite being tested will see. 
The NASA GEVS standard requires that:

"...thermal-vacuum testing at component, subsystem, and system levels of assembly. Eight (8) thermal cycles are required on all hardware prior to assembly on the spacecraft. Normally four (4) cycles are performed at the component level and four (4) at the subsystem or instrument level. Four (4) cycles are also performed on the spacecraft making the total number of thermal cycles twelve (12). The major changes for thermal testing, other then clarifications, are to recommend a $5^{\circ} \mathrm{C}$ margin for acceptance testing and to increase the required margins and durations if tests are approved by Goddard to be performed at ambient pressure. Many Goddard projects already impose the $5^{\circ} \mathrm{C}$ margin for acceptance testing in vacuum. Recommendations are being made to increase test margins by an additional $15^{\circ} \mathrm{C}$ and to increase the number of cycles and dwell times by $50 \%$ if testing is performed at ambient pressure. ${ }^{.5}$

The thermal vacuum testing and qualification of the $\mathrm{CP}$ satellites ensured that GEVS requirements are met both in cycles on the spacecraft along with performing the necessary thermal dwells or "soaks" at both hot and cold extremes. Since testing on the CP satellite was performed in a thermal vacuum at very low pressure $\left(10^{-5} \mathrm{Torr}\right)$ the last portion calling for a $15^{\circ} \mathrm{C}$ margin increase does not apply.

In addition to testing the operation of the spacecraft, the hot dwell or soak periods during these thermal cycles provided the necessary assurance to launch 
providers that the total mass loss (TML) and collected volatile condensable materials (CVCM) values are within spec prior to being integrated onto the launch vehicle and placed next to the primary mission. NASA's Goddard Space Flight Center lists a TML of $1 \%$ or less and a CVCM of $.1 \%$ or less is acceptable and most launch contractors levy this requirement upon their secondary payload providers (it is also a requirement per the CubeSat Design Standard, Revision $12)^{6}$. This standard for outgassing and removing materials is set to follow the ASTM standard which specifies that the vacuum and temperature for performing TML and CVCM testing should be less than $5 \times 10^{-5}$ Torr and $125^{\circ} \mathrm{C}$, respectively.

It is through this rigorous system level thermal testing that CP currently verifies their satellites will operate nominally on-orbit. To demonstrate just how critical this testing is, figure 7 shows the weighted effectiveness for environmental screens (tests) established by the Johns Hopkins APL Space Department, using data compiled from major United States satellite manufacturers. 


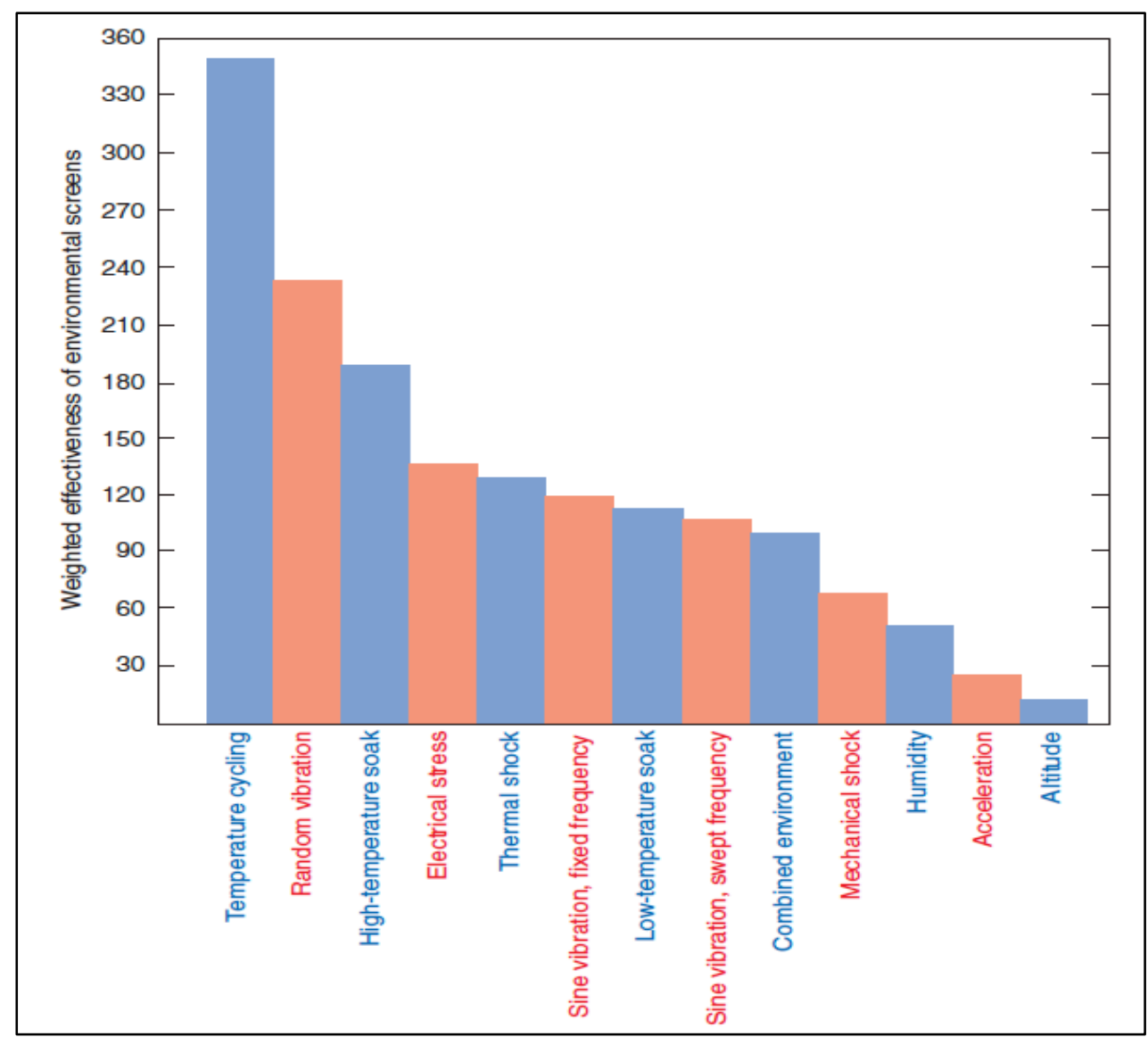

Figure 7. Weighted Effectiveness of Major Spacecraft Tests ${ }^{7}$

Thermal cycling, of the same type applied to all CP CubeSats, is the most effective satellite test method for revealing system issues by a wide margin $(52 \%)$ over random vibration. The additional point that the Johns Hopkins team noted from this chart is that of the entire set of environmental conditions a satellite will see, thermal cycling is the most effective at detecting issues. Also of note was that the hot soak of the satellite ranked third and the thermal shock test ranked fourth, both are also tests that the CP team performs on their operational satellites. 


\subsection{Defining the Unknowns, Material Properties and Variations}

Thermal analyses, even simple ones, are typically very reliable for giving confidence that your satellite design will operate in its expected environment. The unique thing about CubeSats in terms of thermal analyses is that the majority of satellite thermal analysis is based on making engineering assumptions and problem simplifications. Those simplifications and assumptions limit the accuracy of the analysis results. For this thesis to be conclusive there needed to be greater definition and the least number of simplifications made. The starting point was recognizing the unknowns that would have the greatest affect on the analyses in question and putting time and effort into reducing them.

Spacecraft thermal analysis hinges upon radiation as the sole method of heat flux into and out of the system, as such the surface properties of the satellite are critical to be able to accurately predict the systems thermal equilibrium values.

\subsubsection{6-Layer Flame Retardant \#4 Printed Circuit Boards (PCBs) Radiative Properties}

CubeSats made almost exclusively of populated FR4 boards meeting the Personal Computer 104 (PC/104 or PC104) mechanical and electrical standards. PC104 is a natural fit for CubeSats. Literally the PC104 standard calls for a $96 \mathrm{~mm} \times 90 \mathrm{~mm}$ (3.55inx3.75in) board with no backplane and it fits the CubeSat $10 \mathrm{~cm} \times 10 \mathrm{~cm}$ standard perfectly. In lieu of a backplane like your typical Advanced Technology Extended (ATX) or micro ATX (mATX) PC standard, 104 allows individual boards to be stacked together using their 104 pin standard 
interface point and also using standard corner fastener areas to create a "block" PC. This standard was initially designed for specialized embedded computing and customized rugged PC systems for military or industrial applications.

Being designed for embedded computing made PC104 commercial off the shelf (COTS) parts easily available to find and adapt for CubeSat use. Another key driver behind using PC/104 was the relaxed bus drive current of $6 \mathrm{~mA}$. This effectively lowers power consumption to under 1-2 Watts per module and minimizes component count. ${ }^{8}$ The height interface between boards is also set in the standard to be $11 \mathrm{~mm}$ but is not always adhered to, especially for boards that may be using the mechanical standard but not the electrical pin standard.

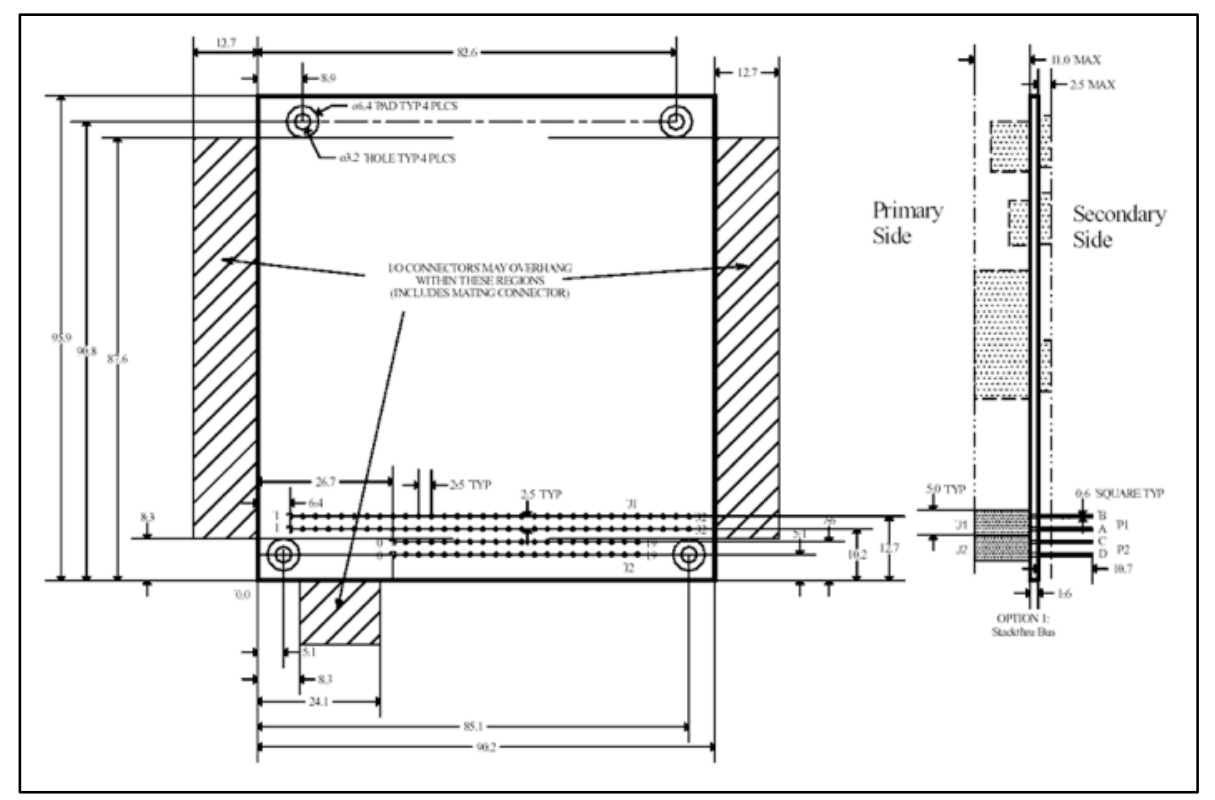

Figure 8. PC104 Standard Board Layout and Mechanical Requirements ${ }^{8}$

Another view of the PC104 standard is shown in figure 9. Here you can see that the stacking utility designed into PC104 is akin to the necessary stacking 
of electronics to efficiently meet the CubeSat Design Standard (CDS).

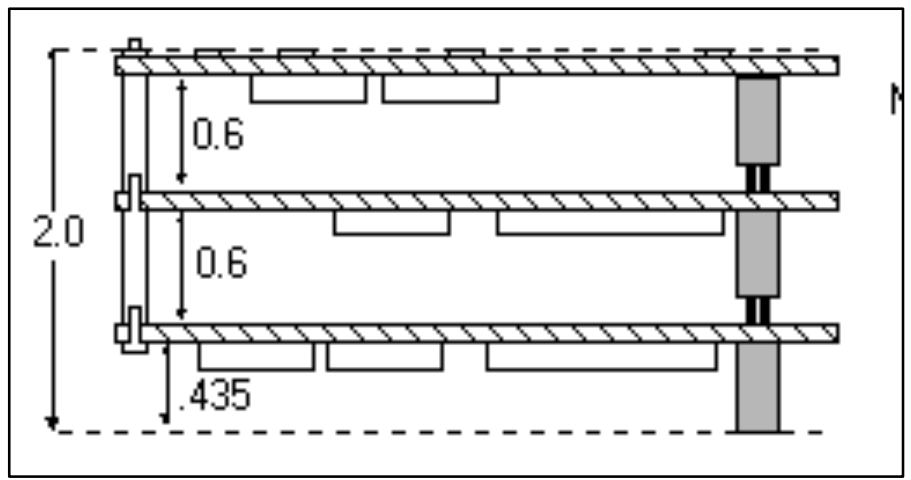

Figure 9. PC104 Horizontal Layout Example ${ }^{8}$

As an example the NPS CubeSat utilized the 104 standard fully, integrating the 104 pin design into their stack shown in figure 10.

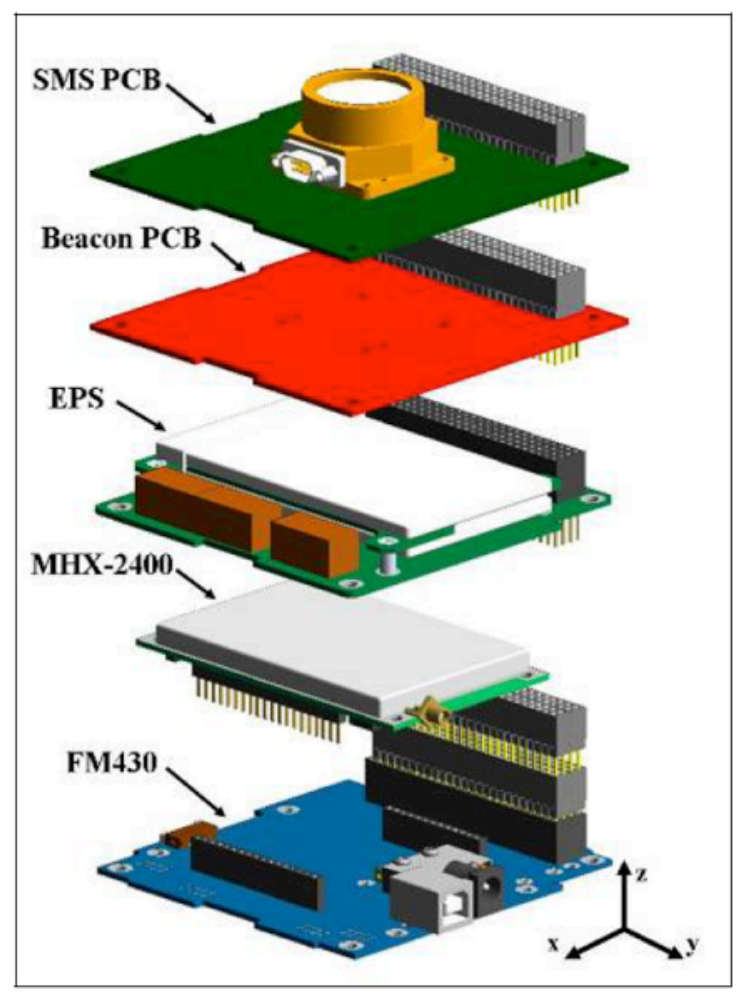

Figure 10. An NPS CubeSat PC104 Stack ${ }^{9}$ 
Comparing the PC104 Horizontal Layout Example in figure 10 to the CP2 "Bus" System Stack in figure 11, along with a clamshell open picture of the CP3 stack in Figure 12, the reason for utilizing the standard is clear. However the CP bus, unlike the NPS-Pumpkin design, doesn't follow the electrical portion of the standard, instead opting for ribbon cabling and alternate board connectors since the amount of necessary board interplay both physical and electrical was found to be more efficient in this manner (for the CP bus specifically).

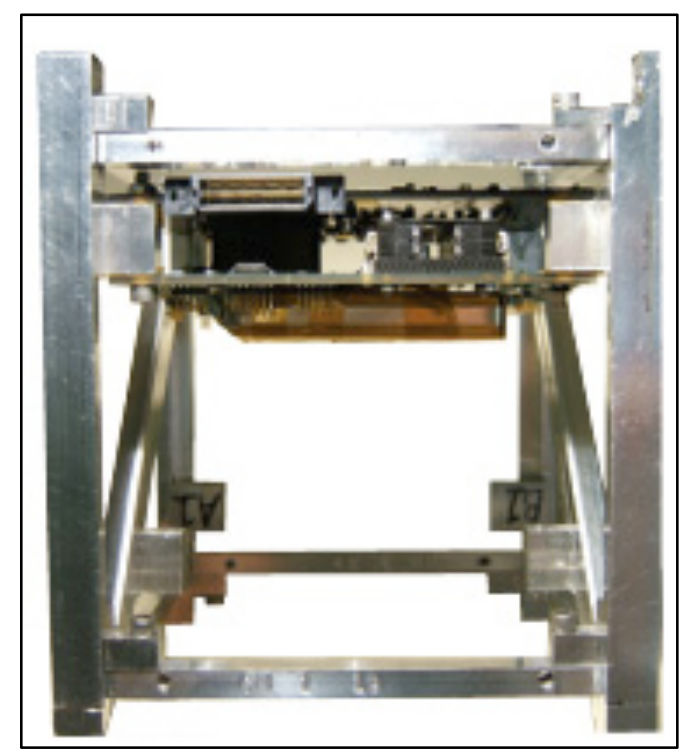

Figure 11. CP2 "Bus" System Stack 


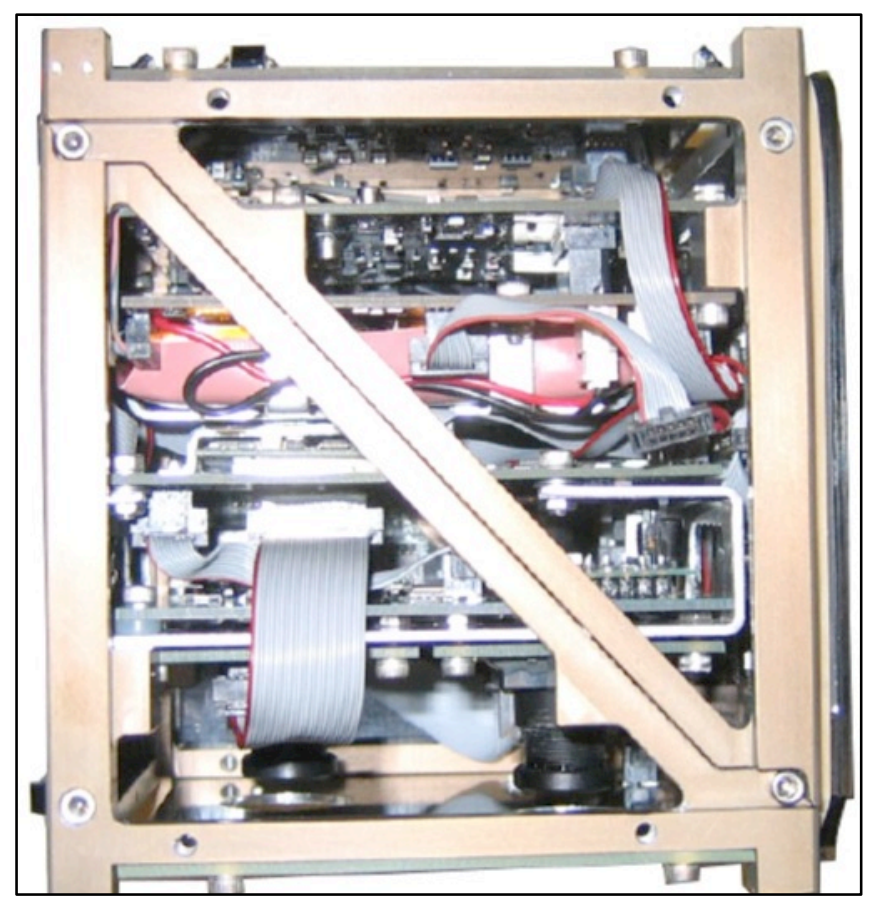

Figure 12. CP3 Bus and Payload System Stack

For reference, FR4 is electrical-grade, fire rated dielectric fiberglass laminate epoxy resin with a glass fabric substrate. The abbreviation FR4 comes from F for flame and $\mathrm{R}$ for retardancies with the 4 denoting a \#4 epoxy. FR4 offers excellent chemical resistance and electrical properties under varied conditions. The standard for FR4 is MIL-I-24768/27.

The PC104 boards used in CP2 and CP3 with no traces, vias or populated components are fairly easy to model for thermal properties and analysis, simply utilize the solder mask surface properties and the well-established FR4 material conduction properties. However that is not at all representative of how the PCBs are actually implemented on the spacecraft. On the spacecraft they are not blank boards, they are densely populated with components of varying size and surface 
properties, none of which is identical to that that of the PCB itself. All of this leads to the first major unknown property that had to be defined - the radiative properties of populated PCBs.



Figure 13. Populated CP2 Power (EP\&H) Board

By reviewing the available area, measuring the laid components and gathering the surface properties of the laid components the average values can be obtained through area calculations. Initially this review began as attempting to count, measure and surface average every component. After attempting to do this for the CP CD\&H board, shown in figure 13 , this was quickly realized to be impractical. In lieu of measuring and cataloging every unique component on the populated board individually the following generalizations were made. These generalizations allowed for a more expedient process while still providing appreciably enhanced surface property knowledge. 
Surface mount resistors are assumed to be uniformly of the metric 1005 standard classification thus establishing a standard area for resistor body and leads of $1.0 \mathrm{~mm} \times 0.5 \mathrm{~mm} \times .35 \mathrm{~mm}(\mathrm{~L} \times \mathrm{W} \times \mathrm{H})$ as shown below in figure 14. The exposed leads are assumed to take up $.25 \mathrm{~mm} \times .5 \mathrm{~mm}(\mathrm{~L} \times \mathrm{W})$ on both ends of the resistor but in the surface area calculations were given no height value since they "drop" when soldered and to a varying degree become flush with the board.

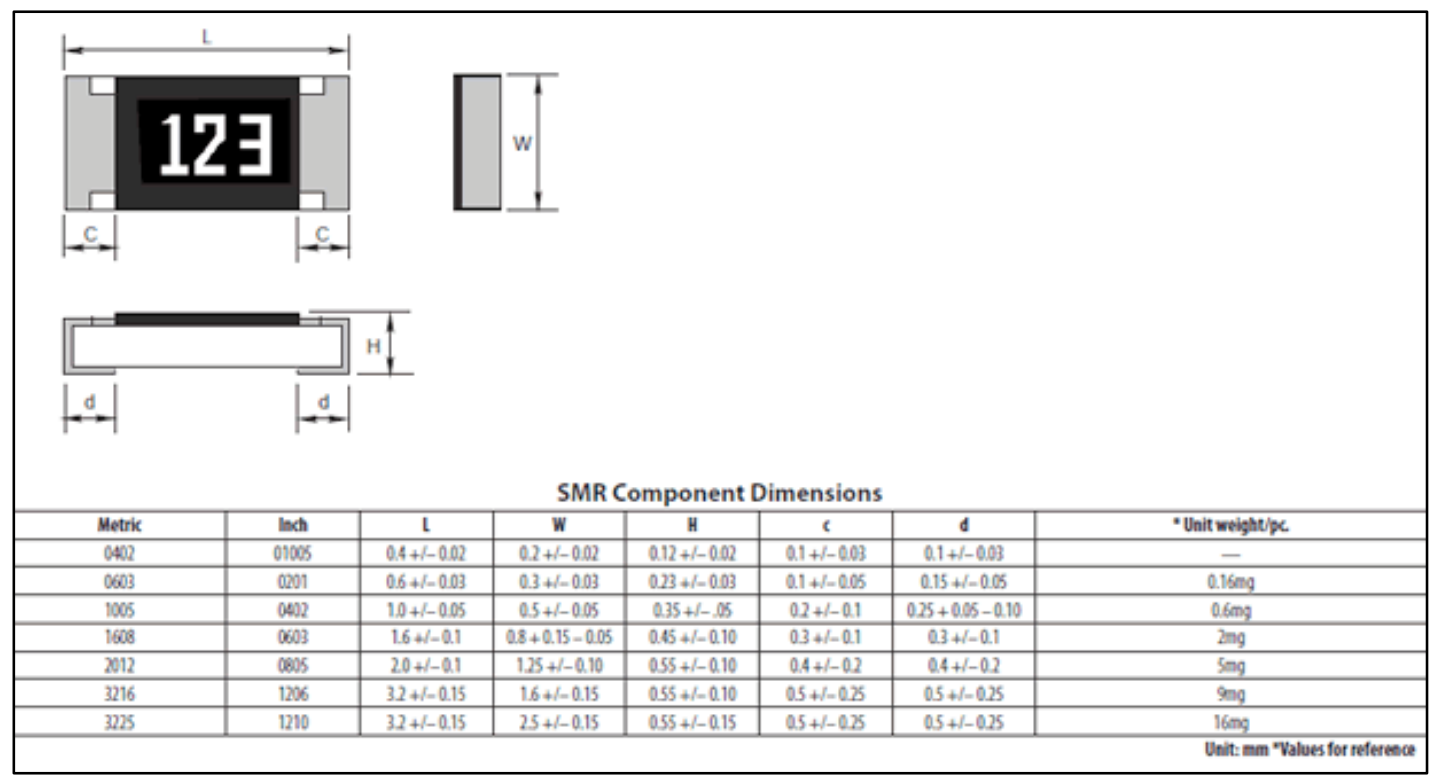

Figure 14. Surface Mount Resistor Size Values ${ }^{10}$

Surface mounted capacitors and similarly sized component are assumed to be uniformly of the metric 1005 standard classification thus establishing a standard area for capacitor body and leads of $1.0 \mathrm{~mm} \times 0.5 \mathrm{~mm} \times 0.5 \mathrm{~mm}(\mathrm{~L} \times \mathrm{W} \times \mathrm{H})$ as shown in Figure 15. The exposed leads on the capacitor are assumed to take up $.25 \mathrm{~mm} \times .5 \mathrm{~mm}(\mathrm{~L} \times \mathrm{W})$ on both ends but in the surface area calculations were given no height value since they too "drop" when soldered and to a varying degree become flush with the board. 


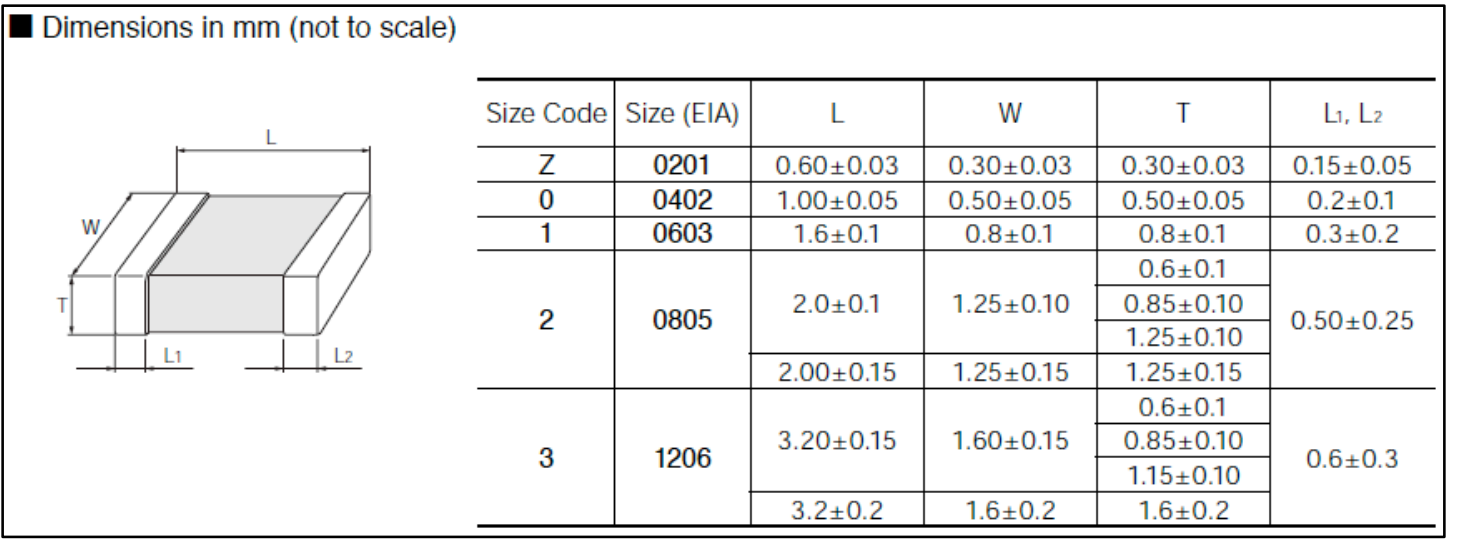

Figure 15. Surface Mount Capacitor Size Values ${ }^{11}$

Surface mounted Maxim 1239 Analog to Digital Converters (ADCs) and similarly sized components were assumed to conform to the nominal (averaged between max-min) values provided for the E16-4 package outline shown below. The averaged value was calculated to be $3.9 \mathrm{~mm} \times 4.89 \mathrm{~mm} \times 1.55 \mathrm{~mm}(\mathrm{~W} \times \mathrm{L} \times \mathrm{H})$. The exposed leads were calculated to each be $1.06 \mathrm{~mm} \times .25 \mathrm{~mm}$ with 8 leads per side (total surface area of $4.24 \mathrm{~mm}^{2}$ per chip). 


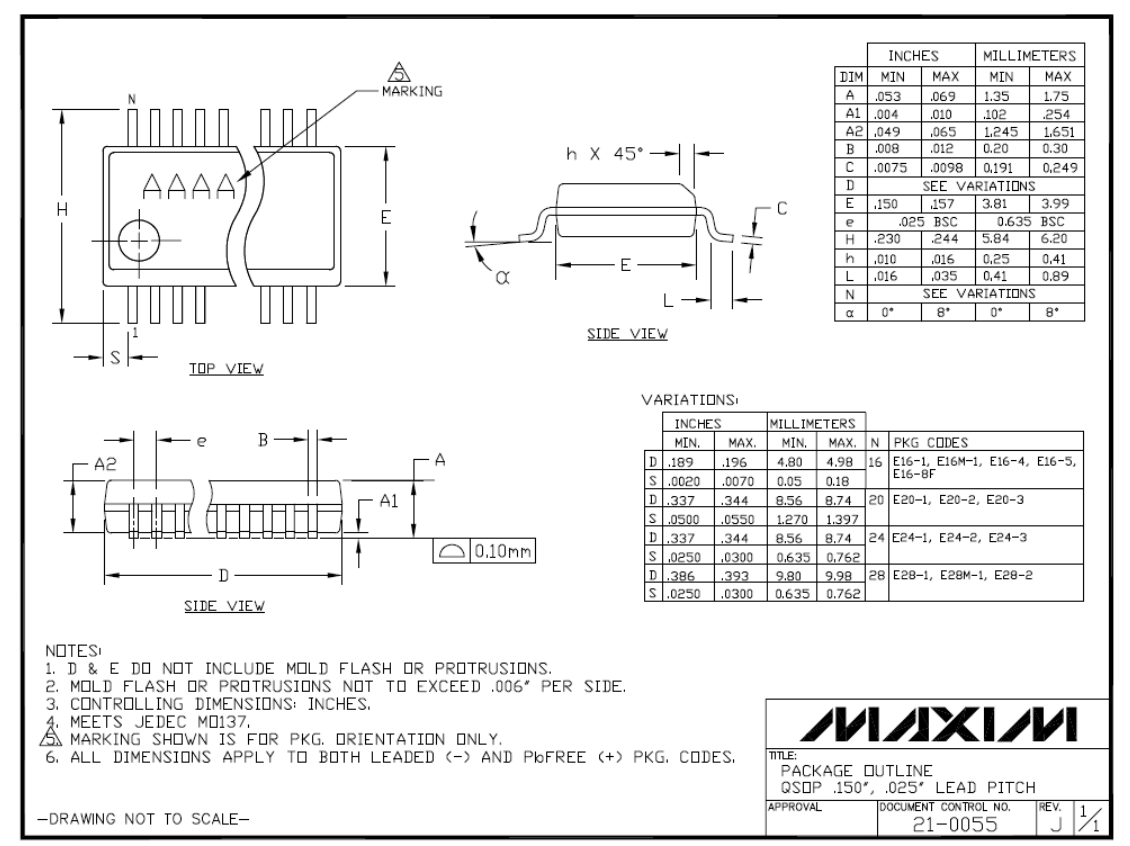

Figure 16. Maxim 1239MEEE Package E16-4 Outline ${ }^{12}$

Surface mounted AD627 instrumentation amplifiers were assumed to conform to the nominal (averaged between max-min) value given in their specification below. The averaged value was calculated to be $3.9 \mathrm{~mm} \times 4.90 \mathrm{~mm} \times 1.55 \mathrm{~mm}(\mathrm{~W} \times \mathrm{L} x$ $\mathrm{H})$. The exposed leads were calculated to each be $1.05 \mathrm{~mm} \times 0.41 \mathrm{~mm}$ with 4 leads per side (total surface area of $3.44 \mathrm{~mm}^{2}$ per chip). 


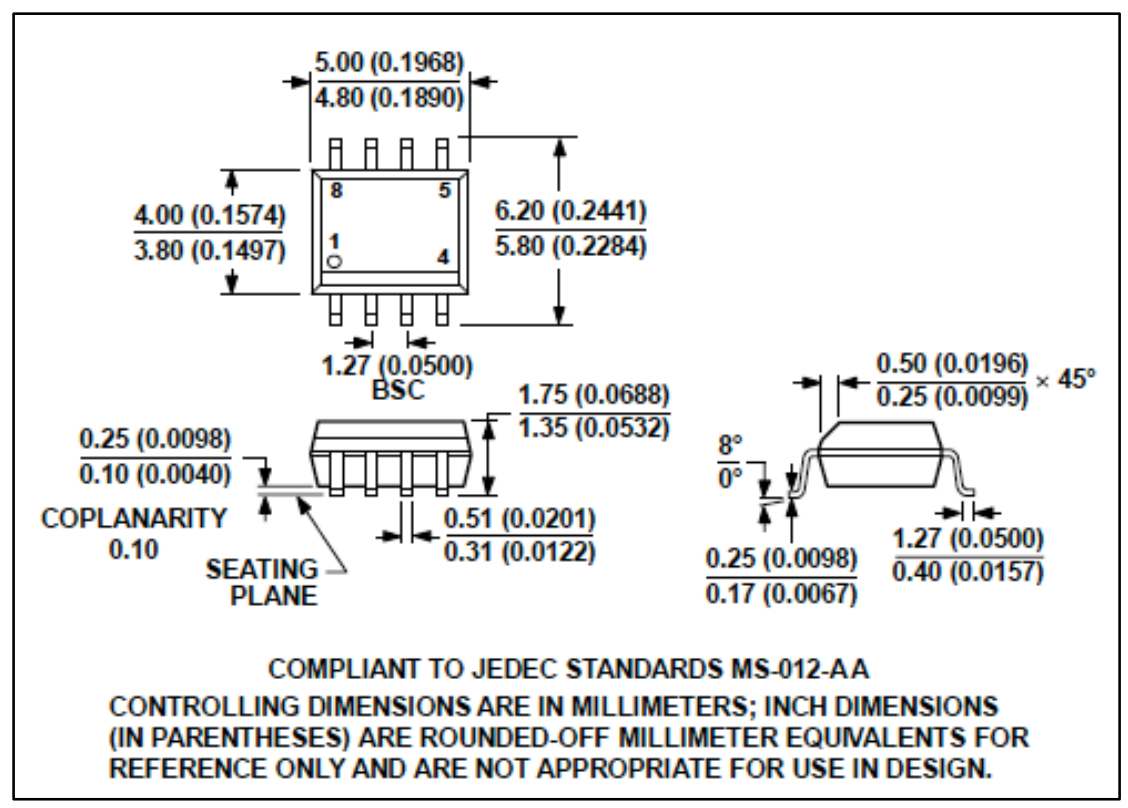

Figure 17. AD627AR Package Outline and Dimensions ${ }^{13}$

With these simplifications the majority of board components could be counted from their layout drawings and the appropriate reductions in solder mask surface area and increases in component surface areas could be accounted for (See Appendix D). With these values calculated a more accurate surface radiative value and area value was obtained. As an example of the discrepancy in both area and radiative properties between a PCB with assumed values and a PCB with adjustments for component population is shown below. 
Table 4. Populated PCB Area and E/Alpha Adjustments - Side Panel Interior

\begin{tabular}{|c|c|c|c|c|}
\hline $\begin{array}{l}\text { Solar Panels } \\
\text { Interior }\end{array}$ & "Resistors" & "Capacitors" & "Maxims" & "ADCs" \\
\hline \multirow[b]{2}{*}{ Extra Surface Area } & 48.00 & 35.00 & 4.00 & 3.00 \\
\hline & 50.40 & 52.50 & 109.00 & 81.84 \\
\hline Replaced Surface Area & 36.00 & 26.25 & 110.20 & 82.65 \\
\hline Avg. Emissivity (Extra) & 0.55 & 0.61 & 0.90 & 0.90 \\
\hline Avg. Emissivity (Replaced) & 0.61 & 0.61 & 0.63 & 0.67 \\
\hline Weighted E By Area (Extra) & 27.55 & 31.85 & 98.10 & 73.66 \\
\hline \multirow[t]{6}{*}{ Weighted E By Area (Repl) } & 21.84 & 15.93 & 69.33 & 55.13 \\
\hline & TOTAL (mm²) & TOTAL $\left(\mathrm{m}^{\wedge} 2\right)$ & \% of Panel & $\%$ of Panel \\
\hline & $\begin{array}{l}293.74 \\
255.11\end{array}$ & $\begin{array}{l}0.00029 \\
0.00026\end{array}$ & & $7 \%$ \\
\hline & E AVG (SA) & E AVG (EX + RE) & New $E$ & Previous E \\
\hline & 0.79 & 0.72 & 0.81 & 0.90 \\
\hline & 0.64 & & & $10 \%$ \\
\hline
\end{tabular}

For the side panel interiors which re-radiate heat to the interior of the spacecraft the difference between the previous emissivity value and the corrected populated PCB value was $10 \%$ and changing from .90 to .81 . The table summary for exterior side panels is below.

Table 5. Populated PCB Area and E/Alpha Adjustments - Side Panel Exterior (Solar

Panels)

\begin{tabular}{|lcccc|}
\hline Solar Panels & Cells & \multicolumn{2}{c|}{ Leads } \\
Exterior & 2 & 6.00 & & \\
\hline & 54.6 & 2.32 & Averaged & $\%$ Change \\
Surface Area & 0.85 & 0.02 & 0.82 & $4.0 \%$ \\
Avg. Emissivity & 0.92 & 0.3 & 0.89 & $2.7 \%$ \\
Avg. Absorptivity & Remainder of Panel is Soldermask @.9 & & 0.84 \\
Averaged E For Panel & \multicolumn{5}{c}{} \\
\hline
\end{tabular}

For the solar panels the change was not as large but was still numerically significant (as shown in section 3.2.4 results) going from an emissivity of .85 to 
.82 and an absorptivity of .92 to .89 showing a discrepancy of $4 \%$ and $2.7 \%$ respectively.

On the C\&DH PCB the change was even greater than on the side panel interior. In Table 6 below the difference was found to be a $16 \%$ total reduction in the emissivity value for the surface. Since this board is by far the most populated, with component surfaces making up $12 \%$ of the actual panel surface area, it was not surprising to have that large of a change, but it illustrated again that assuming the base material emissivity (.90) for the entire panel is not a conservative nor an accurate assumption that CubeSat thermal designers should make. The actual emissivity value of .76 is significantly lower than the .90 and will result in less energy exchanged to and from the side-panel interiors (which also had a lower true emissivity value).

Table 6. Populated РСВ Area and E/Alpha Adjustments -C\&DH Board (Satellite Interior)

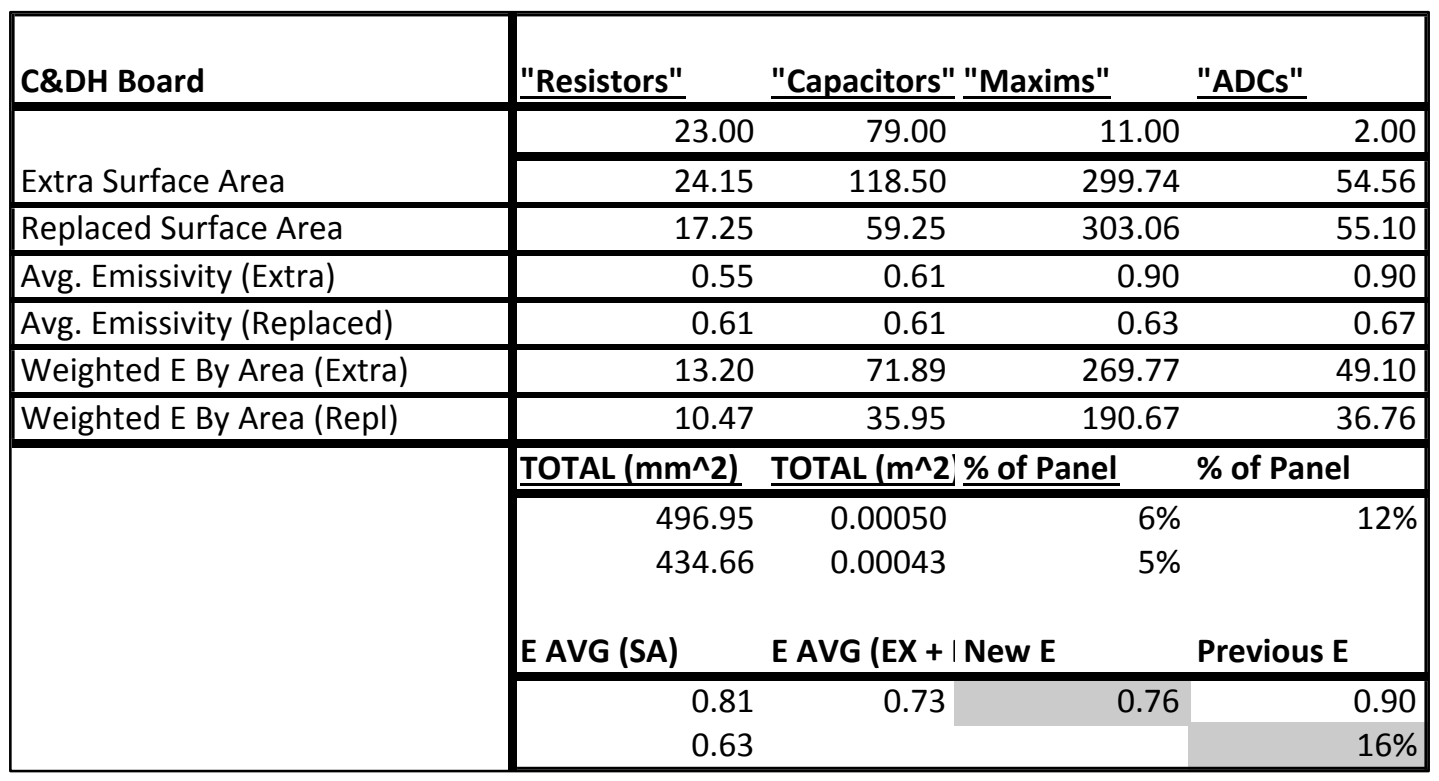




\subsubsection{TAIYO PSR-4000BN Liquid Photoimageable Solder Mask Radiative Properties}

The next set of unknowns that required testing or analysis to define was the IPC-SM-840 compliant TAIYO Solder Mask utilized to coat the PCB surfaces. Solder mask is a thin layer of deposited epoxy liquid that is silkscreened onto the finished PCB pattern. It protects the otherwise exposed top layer copper traces (CP3 uses a 6-layer PCB) from damage while also preventing solder from spanning conductors and causing a short. Other than the exterior of the solar panels this is the dominant surface material in the satellite.

As this material plays such a significant role in the heating and cooling of the satellite it was critical to have accurate knowledge of its properties. The available information was limited, the PCB supplier was unable to find or provide anything beyond the TAIYO specification sheet that showed compliance with IPC-SM-840 but provided little else in terms of applicable surface properties (Appendix E). TAIYO was contacted several times via phone and email but were not willing to provide anything beyond their specification sheet which had already been provided via Sierra Proto Express.

After research on several manufacturers websites it was discovered that while certain manufacturers quoted surface properties they varied by spectrum and also varied by manufacturer. Only properties for green solder mask were provided as it was the most typical application. CP boards used a mix of green, 
red and black solder masks with black solder mask being the most prevalent and the kind used in the later revisions of the system (such as CP3 and CP6).

\subsubsection{A Thermal Vacuum Test Method for Obtaining Emissivity Values}

Two tests were designed and performed specifically to categorize the solder masks emissivity and absorptivity with accuracy. The first test followed the method prescribed by Dr. Ross Judd from the University of Michigan in his article "Determination of the Emissivity of a Heated Copper Surface."14

Dr. Ross' method uses the principle that the rate at which heat is transferred from the surface of a heated object will become equivalent to the rate at which heat is generated within the object when steady state conditions have been attained..$^{14}$ If the associated conduction and convection heat transfer effects can be eliminated then radiation heat transfer becomes the only method available to the heat flux.

The proportion of radiant thermal energy that is transferred to and from an object by radiation can be objectively quantified using the object's radiant properties. These properties can be estimated using tables, calculated using material properties and surface roughness or obtained through experimental processes. The most accurate, though work-intensive, method is typically experimentation. In order to properly quantify the emissivity value of the solder mask on the PCB panels a thermal vacuum test was designed following Dr. Ross' method. 
By utilizing a thermal vacuum chamber one can nearly eliminate heating by conduction or by convection. While the thermal vacuum chamber is at or near $6 \times 10^{-5}$ Torr there is so little gas available that convection or even molecular conduction through it is effectively negated. The layout of the test is shown in Figure 18 below for reference where the board is placed upon four Teflon standoffs to isolate it from the solid copper baseplate and then thermistors were placed at three locations along the board in a diagonal $(1,2,3)$. Also the standard thermistors used by the Omega thermal controller are shown relative to the thermal shroud as $(4,5)$.

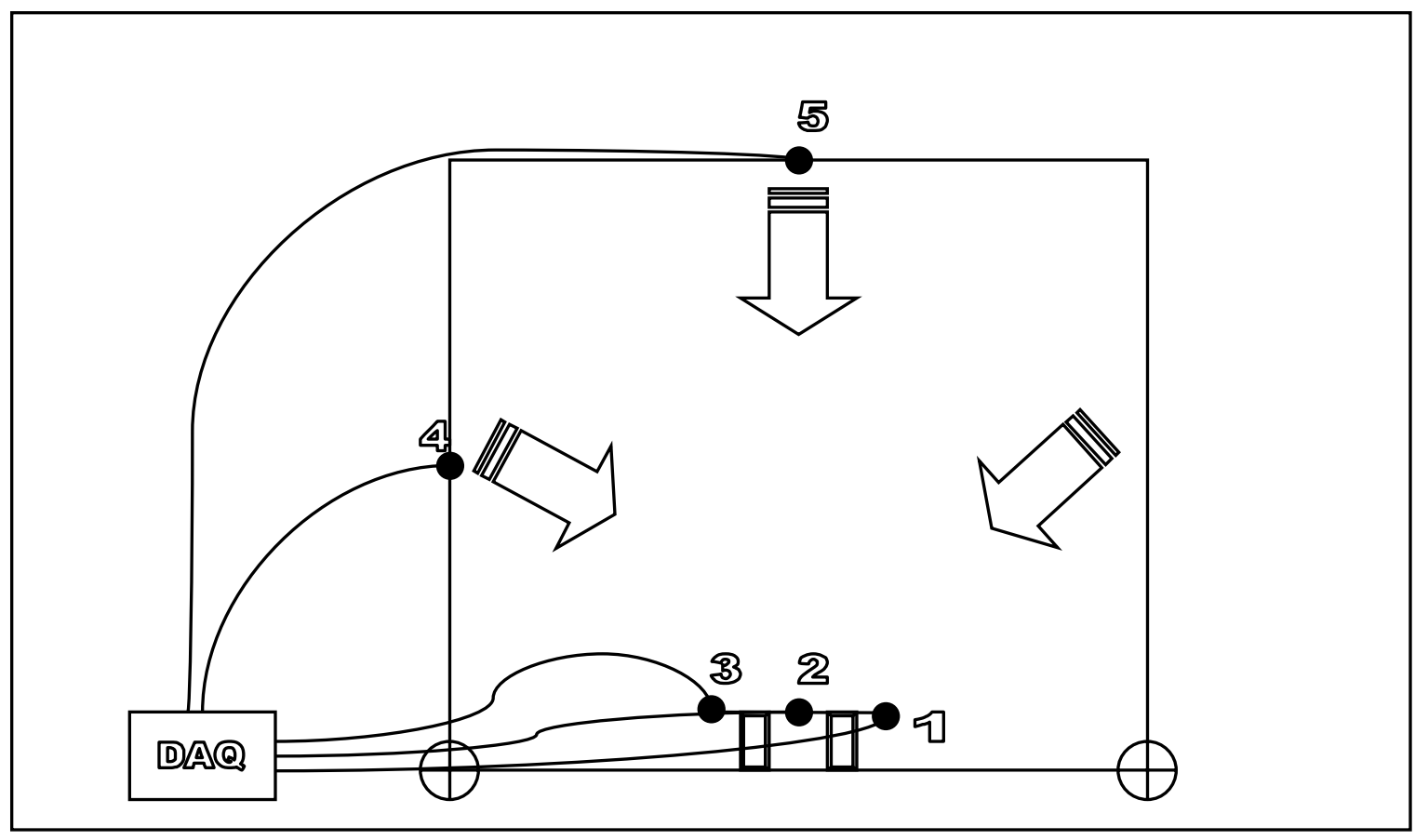

Figure 18. Emissivity/Absorptivity TVAC Test with Thermistor Locations

Figure 18 illustrates the location of the panel being tested. The panel has been isolated from the thermal enclosure using circular solid Teflon (Polytetra 
Flouroethylene) standoffs. The Teflon standoffs are extremely isolative and have very low conduction. The total heat transfer through conduction in the Teflon was calculated to ensure that the amount of heat being transferred was truly negligible.

$$
q^{k}=-k \frac{\partial T}{\partial n}
$$

\section{Equation 1. Fourier's Law of Heat Conduction}

The difference in thermal conductivity between the two temperature extremes that the Teflon was exposed to was found to be minimal and so the thermal conductivity was considered constant for the duration of the experiment.

An adjusted form of the Fourier Law of Heat Conduction with the associated material conductivity is shown in Equation 2 below where $Q$ is the total heat conducted per time:

$$
\frac{Q}{t}=\frac{k A\left(T_{H}-T_{C}\right)}{d}
$$

\section{Equation 2. Fourier Law of Heat Conduction With Conductivity Values}

Using the values for the Teflon TFE standoffs where $\mathrm{k}=.002 \mathrm{~W} / \mathrm{m}^{2} \mathrm{C}^{\circ}$, then assuming a worst-case temperature dispersion between the shroud (hot) and the panel (cold) and lastly filling in the required area and length values generates the equation below. 


$$
\frac{Q}{t}=\frac{.002 W^{*} .0113 m^{2}\left(86^{\circ} C-20^{\circ} C\right)}{m^{2} C(.0032 m)}
$$

\section{Equation 3. Solved Conduction Equation for Standoffs}

From the results of the calculation the assumption of negligible conductive heat transfer in comparison to the amount of heat flux generated by the enclosure's resistive heaters holds true.

The thermal enclosure is made of 8-gauge electrical-grade copper with a one-inch thick copper baseplate. There are three 400-Watt resistive heaters grounded to each wall of the shroud (top, left side, right side) and attached to an Omega 300A thermal controller.

The DAQ (Data Acquisition) interface is located at the lower left corner, exterior to the thermal enclosure but interior to the thermal vacuum chamber. Here the thermistors are all brought through a sealed plate to the exterior of the chamber where they are fed to the Omega controller and reported via a USB link, tracked and saved via a LabView interface to a computer exterior to the cleanroom staging area.

Three interior thermistors were $(\# 1,2,3)$ placed on different locations of the panel. Two thermistors are permanently located on the shroud itself which are utilized as controller temperatures for the Omega temperature controller system. The shroud also contains four machined Teflon offsets that isolate the thermal enclosure from conduction to the stainless steel interior mass of the thermal 
vacuum chamber itself. These are seen on the diagram above at the lower right and left corners of the thermal enclosure. One of the corner standoffs can also be seen in figure 19 below. Note the image to the left is taken from prior to the installation of the thermal shroud into the thermal vacuum chamber while the image to the right has the shroud installed.
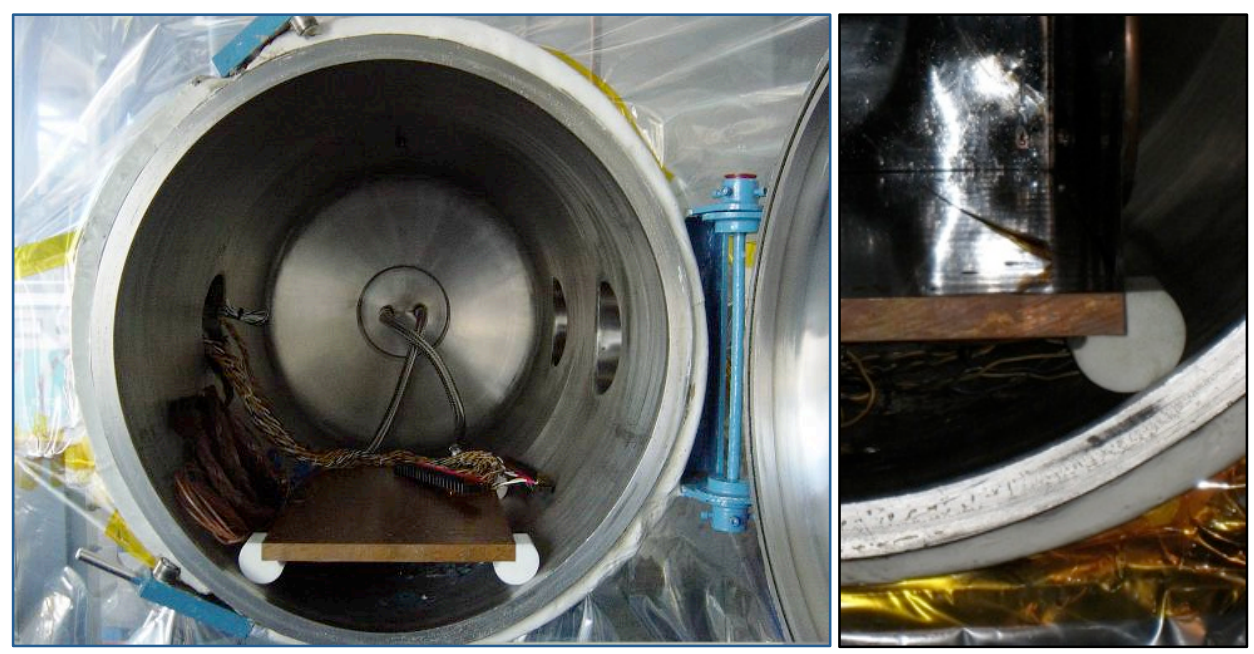

Figure 19. Teflon Thermal Standoff Illustration Pre-Shroud \& with Shroud

The experiment utilizes the fact that both convection and conduction, as shown later, have been essentially removed from the system and the only remaining method of heat transfer is radiation. The equation governing the radiative heat exchange of the surrounding heating elements and copper shroud, total $q_{e}$, is shown below (SP denotes side-panel - CS denotes copper shroud).

$$
q_{e}=2 A_{S P} \varepsilon_{S P} \sigma\left[T_{C S}^{4}-T_{S P}^{4}\right]+q_{c}
$$

\section{Equation 4. Radiative Heat Exchange for Testing}


In this equation $q_{c}$ designates any additional heat transfer occurring due to minor conduction from the standoffs or from the rarified atmosphere between the side panel and the surrounding enclosure. The theory of Kyte, Madden and Piret ${ }^{15}$ conveyed in the graph below enables the prediction of the value of $q_{c}$ for a generic copper sphere in different levels of a vacuum. It is plotted as a function of the copper sphere and the pressure of the air surrounding it.

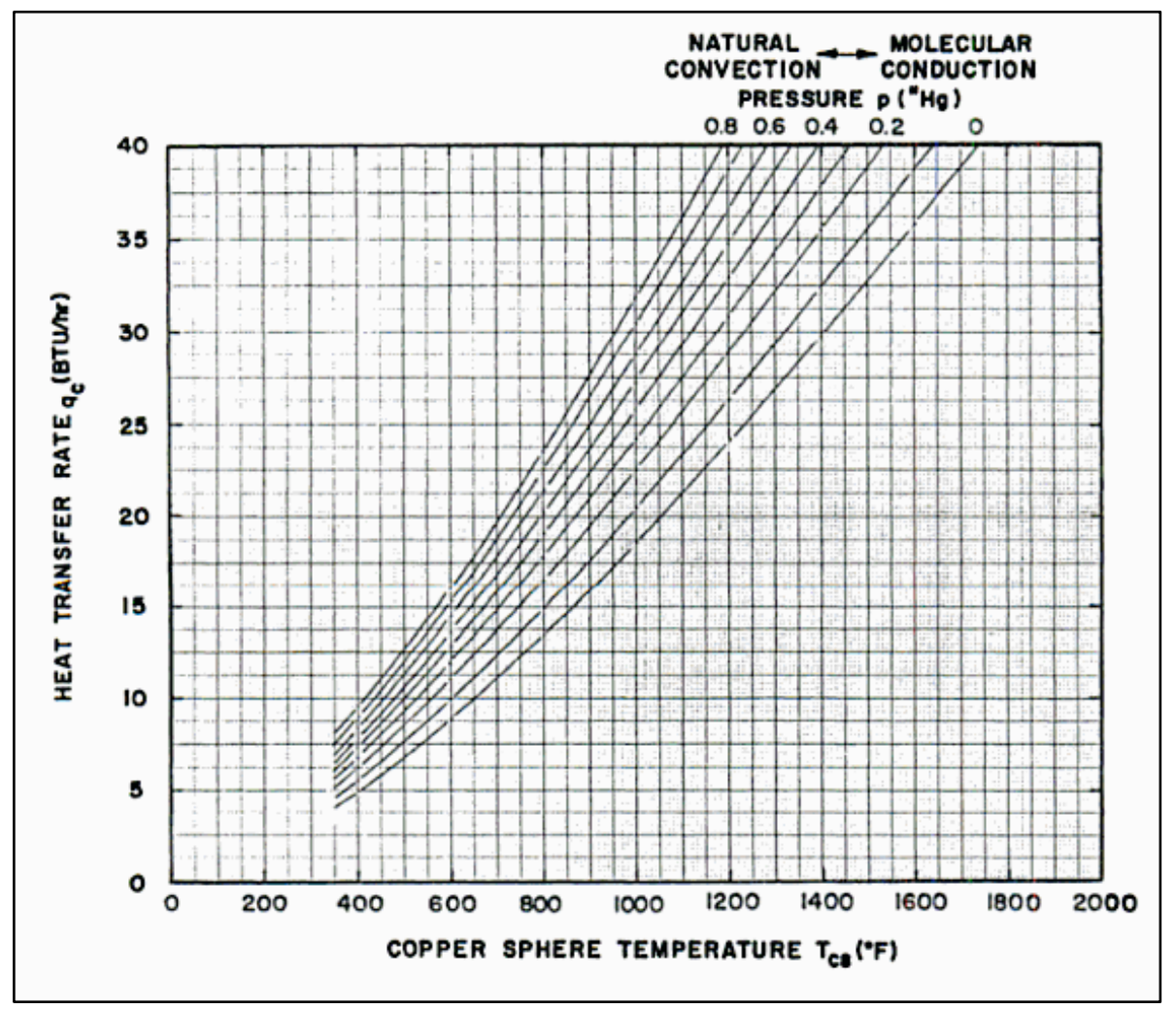

Figure 20. Heat Flux (BTU/hr) vs Test Article $\mathrm{T}_{\mathrm{C}}$ vs Pressure ${ }^{14}$

Since we have proven that the atmosphere is "high-vacuum" and the standoffs provide only negligible conduction, using Kyte, Madden and Piret's theory and data in Figure 20 above we can remove $q_{c}$ leaving the following. 


$$
q_{e}=2 A_{S P} \varepsilon_{S P} \sigma\left[T_{C S}^{4}-T_{S P}^{4}\right]
$$

\section{Equation 5. Reduction of Radiative Heat Transfer using Kyte, Madden and Piret Theory}

The $\varepsilon_{S P}$ designates the emissivity of the side panels (in question) and $\sigma$ $=0.1718 \times 10^{-8} \mathrm{BTU} / \mathrm{hr}-\mathrm{ft}^{2}-\mathrm{R}^{4}$ is the Stefan Boltzman constant. Using our measured shroud and baseplate temperature along with our known heater input we can find the only unknown, the emissivity value.

$$
\frac{q_{e}}{2 A_{S P} \sigma\left[T_{C S}^{4}-T_{S P}^{4}\right]}=\varepsilon_{S P}
$$

\section{Equation 6. Solving Radiative Heat Exchange Equation for Emissivity}

Table 7 shows the experimental values obtained and the emissivity derived for each steady state level that was used.

Table 7. Emissivity Obtained Through Thermal Vacuum Testing Method

\begin{tabular}{|c|c|c|c|}
\hline Pressure & $\mathbf{T}_{\mathrm{CS}}\left({ }^{\circ} \mathrm{K}\right)$ & $\mathrm{T}_{\mathrm{SP}}\left({ }^{\circ} \mathrm{K}\right)$ & ${ }^{*} \mathcal{E}$ SP \\
\hline $\mathrm{P}<10^{-5}$ Torr & 312.95 & 310.75 & 0.80 \\
\hline $\mathrm{P}<10^{-5}$ Torr & 318.25 & 316.35 & 0.88 \\
\hline $\mathrm{P}<10^{-5}$ Torr & 322.75 & 320.85 & 0.84 \\
\hline $\mathrm{P}<10^{-5}$ Torr & 327.95 & 326.05 & 0.81 \\
\hline $\mathrm{P}<10^{-5}$ Torr & 342.95 & 341.55 & 0.95 \\
\hline
\end{tabular}


This method of obtaining the emissivity values for the side panel was found post-testing to be highly inaccurate and from a perspective of increasing the accuracy of the side panel emissivity values it was an extremely time consuming failure. Simplifications that Dr. Ross was able to perform in keeping the flux values constant, the surface areas simple and the view factor at 1 made his experiment more accurate, varying only $9 \%$ between his maximum and minimum results.

The results for the authors experiment varied $16 \%$ from the maximum to the minimum values with the average emissivity being .86 with a standard deviation of .06 . The results were most likely affected by different plateau or step values not being held for long enough to be considered steady state. The last step used was 69.8 degrees Celsius and was held for the longest duration ( 40 minutes) and as such is probably the most accurate value amongst the data set. If a similar test were to be performed in the future increasing the requirements for steady state to be $d T_{\text {Panel }}<1^{\circ} \mathrm{C}$ per 10 minutes instead of the $d T_{\text {Panel }}<1^{0} \mathrm{C}$ per 5 minutes (as was used), would likely decrease the variability in the values obtained. However, increasing the steady state requirements would in turn drastically increase the length of the test beyond the eleven hours already required to complete the thermal vacuum test portion and force the test to be broken into two days, introducing repeatability challenges.

Despite the time invested in trying to experimentally solve for the solder mask emissivity in this manner, the author was forced to find a different way to 
more accurately solve for the emissivity. The next method used was not only faster but also far more accurate, though it required hardware that was not previously available when Dr. Ross' method was attempted.

After performing thermal-vacuum testing and calculating the results for solder mask emissivity, the author recognized that a more accurate testing method would be required. Several experts were contacted including the Raytek office in Santa Cruz, CA. Raytek is a Fluke company that specializes in noncontact infrared temperature measurements. They were able to suggest several products that could support accurately defining the emissivity values of the panels along with a general test-method for finding those values.

\subsubsection{An Improved Emissivity Test Method Via the RAYTEK MI40}

Unfortunately the cost to either purchase an non-contact imager with iterative emissivity support like a Fluke 68 (cost: $\$ 575.00$ ) or rent a non-contact thermal imager like the Fluke ti25 (cost: $\$ 320.00 /$ day + deposit) were very prohibitive to a student budget and timeline. Fortunately an alternative was found. The release of the Ratyek Thermalert MI45, made the MI40 sensor less desirable. With the help of a Raytek sales engineer, a company that had recently purchased the MI45 models to phase out their existing MI40 Thermalert models was found. The author was able to purchase an un-used spare for about one fifth of the OEM price (\$470.00) from the company. As a lesson learned to the CubeSat community, where budgets are always tight, asking companies for older or outdated but roughly equivalent versions is a one way to lower testing 
equipment costs. Some companies, like Northrop Grumman (previously TRW), will actually donate expensive but used equipment like Thermal Vacuum chambers free of charge provided it will be for educational use.

The MI40 sensor acquired is a ruggedized passive IR sensor designed to measure factory process temperatures with a high degree of accuracy $(+/-1 \%$ per Raytek Specification) when a contact sensor is not reasonable. The key to its usefulness for this thesis is that it was the lowest cost available sensor that still had high accuracy and adjustable emissivity. Since the temperature being read via infrared radiation is highly surface dependent, the sensor allows the user to manually adjust the emissivity value to get accurate and repeatable process results. In essence the test necessary to accurately predict the solder mask emissivity was just the reverse of the test procedures that the industrial users require to generate accurate temperature data.

The MI40 has a simple power interface and adjustable output options varying from $\mathrm{J}$ or $\mathrm{K}$ thermocouples to a simpler $0-5$ Volt or $4-20 \mathrm{~mA}$ output. For the purposes of this test the $0-5$ Volt option was used. The unit was connected to a power supply on the test bench in the Advanced Technology Labs and then breakout wires (shown in Figure 21. below) were connected to a lab multimeter. The following settings were adjusted on the MI40 sensor box prior to powering on the unit and beginning the test. The 0-5 V jumper and 4-20 mA jumper were installed leaving the $\mathrm{J}$ and $\mathrm{K}$ thermocouple jumpers open thus setting the unit to 0-5 V output mode. The emissivity was set to .95 as a starting point for our test- 
using figure 3 in the MI40 Operator's Guide, (figure 21) shown below as a reference. The actual setting jumpers can be seen in figure 22 .

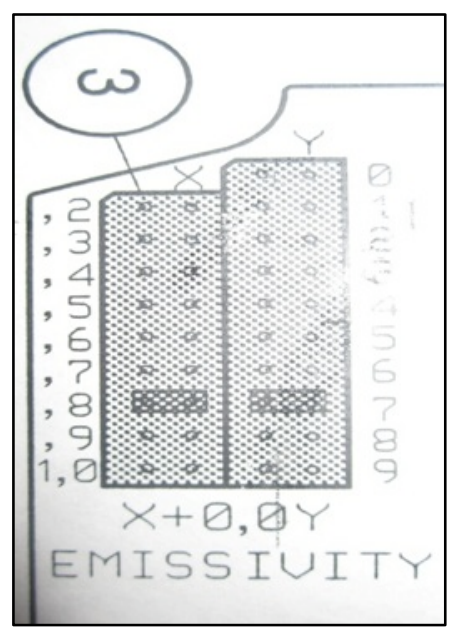

Figure 21. Emissivity Reference Panel from MI40 Operator's Guide

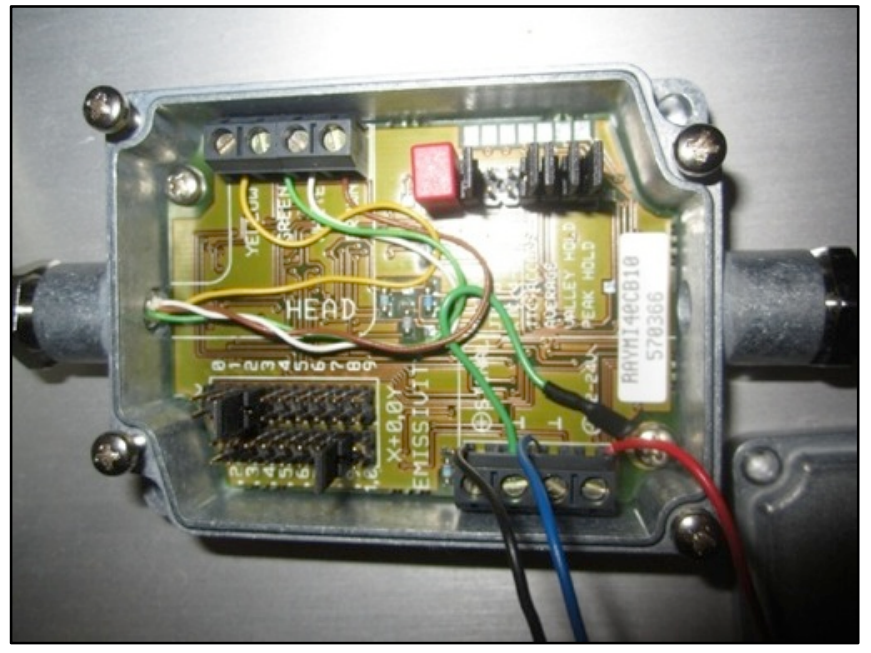

Figure 22. Raytek Thermalert MI40 w/ Breakouts Wires Installed

At this time the test article (CP3 Payload Face board - spare) was laid on the bench and a thermistor was taped with Kapton to the back of the board. The thermocouple (a type $\mathrm{J}$ ) was connected to the multimeter first and the temperature of the sample was verified to be $21.6^{\circ} \mathrm{C}$. Next the thermocouple was 
taped to between the sample (board) and the black 3M electrical tape covering one edge of the board and the temperature was again verified to be $21.6^{\circ} \mathrm{C}$. Next the thermocouple was removed from the multimeter and leads with plunger hook clips were inserted for connection to the output on the sensor.

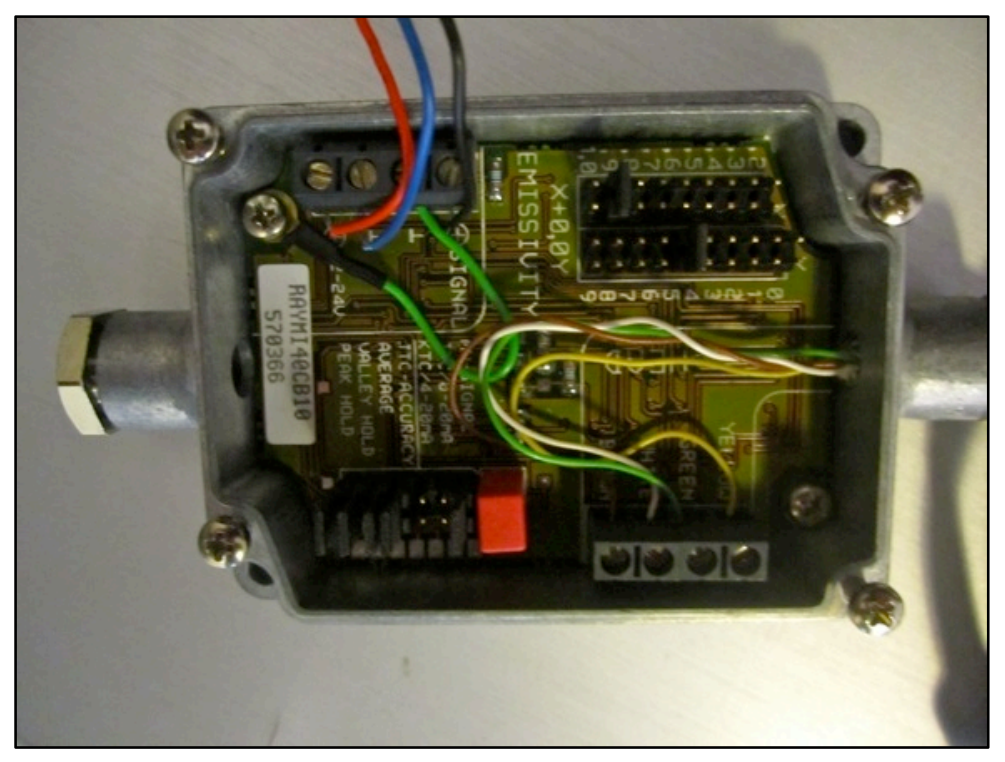

Figure 23. Raytek Thermalert MI40 Post Emissivity Adjustment

At this point the lens cap on the MI40 was removed, while wearing latex nitrile gloves to ensure no molecular deposition occurred, and the sensor head was carefully pointed at the 3M 49656 Black electrical tape portion of the side panel. 

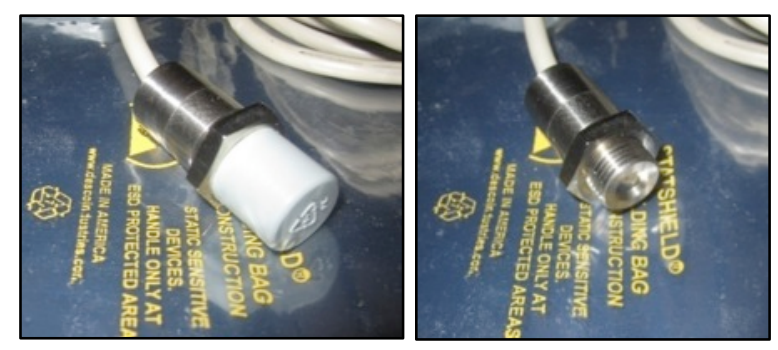

Figure 24. IR Sensor Protective Cover and Cover Removed for Testing

This tape specifically is used because it has a very well defined, tested and documented emissivity value of .95 . Having an accurate known emissivity reference provides an easy way to compare the two materials and accurately find the unknown solder mask emissivity value. In figure 25 below even the light balance has very low reflectance off the taped portion of the board, an easy sign that it is a good emitter. As a side note the adhesive on electrical tape should never be placed on a flight satellite panel as it left significant molecular residue requiring extensive IPA and Kimwipes to clean. This test was done using a nonflight board, but as a reference to other CubeSat teams, do not put this tape on your flight board to calculate its surface emissivity! 


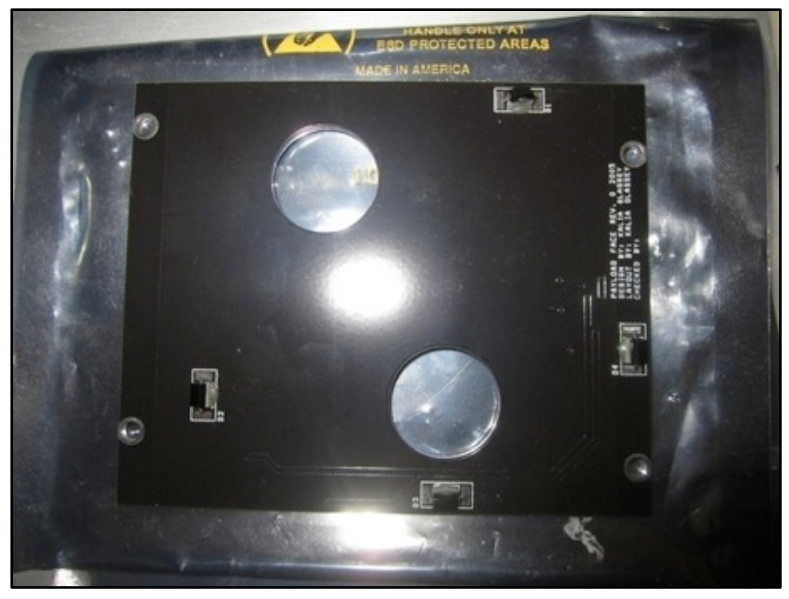

Figure 25. Payload Face Board used for Testing (pre-3M Taping)

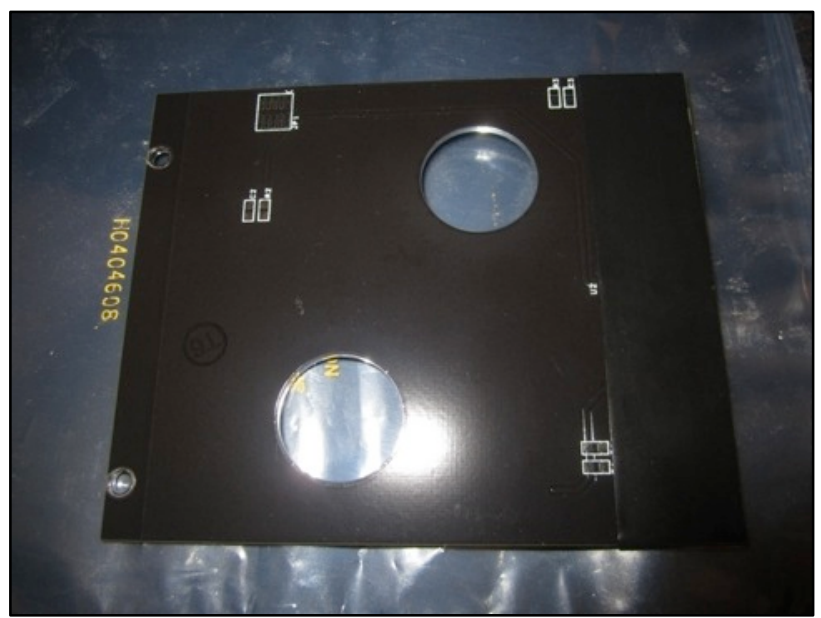

Figure 26. Payload Face Board used for Testing (post-3M Taping)

The voltage was read three times on the multimeter and recorded in a lab notebook. Next the sensor head was pointed at the un-taped portion of the solder masked payload face. Still wearing the latex nitrile kimtech gloves the emissivity gain jumpers were stepped down in .01 increments until the initial measured voltage was reached. This was performed six times, each time rereading the known .95 emissivity tape and then aiming the sensor head at the solder mask, and adjusting the emissivity gain down until the voltage output was 
matched as close as possible to the initial reading. After the third test the thermocouple was reattached to the multimeter and the samples were re-verified to ensure their temperatures were still uniform. This was done at a distance of less than 2 inches away from the material to ensure the sensor heads spot diameter didn't exceed the width of the taped section.

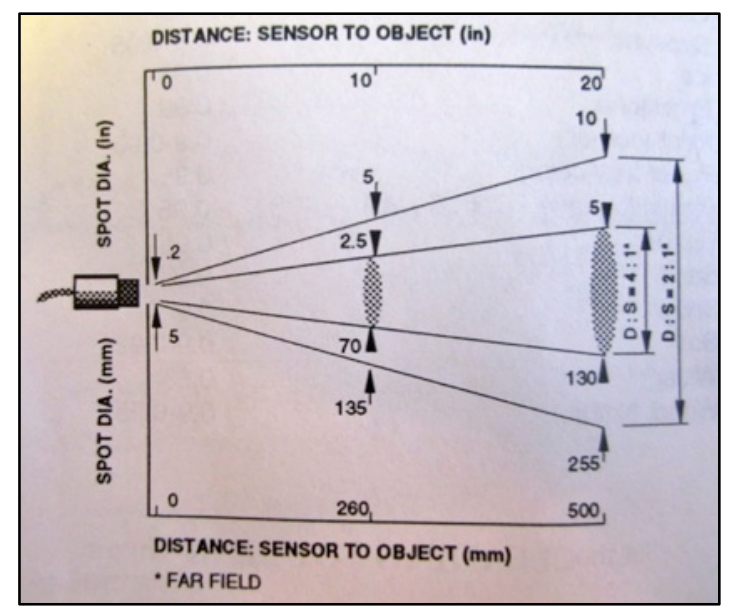

Figure 27. Sensor “Spot Diameter” versus Distance from Raytek Operators Guide.

Post-test the emissivity values were placed into table format and then combined with the initial method to illustrate the improvement in accuracy, the results of this comparison can be found in Table 25 (next page) with the details included in Appendix D. The average of the emissivity settings using this testing method (shown in Table 19 below) was .90 and so this was the value used going forward to represent the black solder mask emissivity. 
Table 8. Solder Mask Emissivity Test Results

\begin{tabular}{|c|c|c|}
\hline Run \# & Sample (Reference) Temperature & Emissivity (Final Gain Setting) \\
\hline 1 & 21.6 & .91 \\
\hline 2 & 21.6 & .90 \\
\hline 3 & 21.6 & .90 \\
\hline 4 & 21.8 & .89 \\
\hline 5 & 21.8 & .90 \\
\hline 6 & 21.8 & .90 \\
\hline
\end{tabular}

While the testing performed to meet Dr. Ross' prescribed method was an excellent albeit time consuming learning experience the final value that would be used for thermal analyses came exclusively from an average of the MI40 testing whereby the emissivity value was established to be 0.90 . This value was within the range of what external sources both online and in print had proposed for solder mask emissive values. Specifically this final value was checked against Clyde Coombs "Printed Circuits Handbook" section 17.11 in which he states "typical solder mask has an emissivity ranging from .85 to $.95 \ldots$ depending on the roughness...." Since our solder mask was a dark color with limited surface roughness this value was very reasonable. 


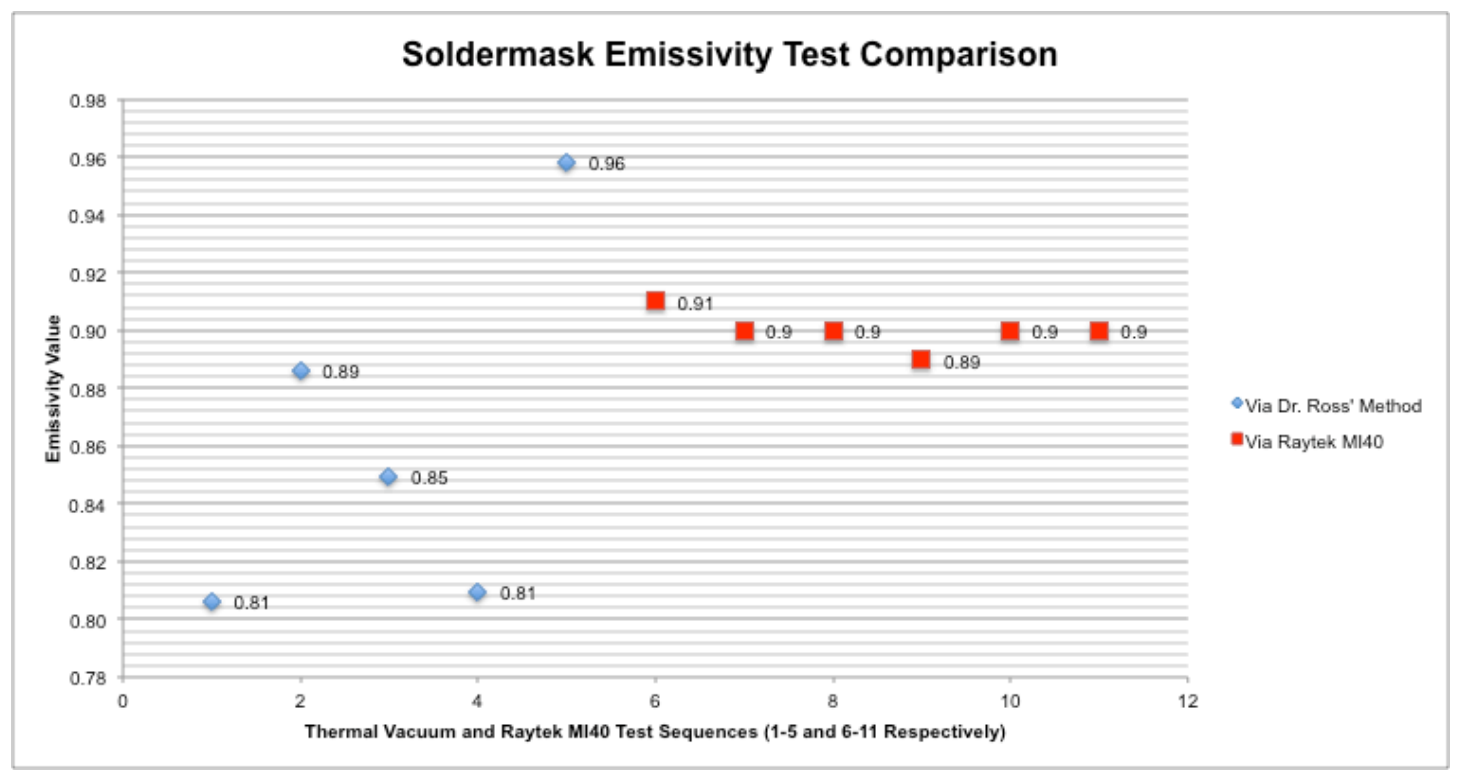

Figure 28. Solder Mask Emissivity Test Comparison

\subsubsection{Conformal Coat (IPC A-610 Compliant) Radiative Properties}

One item that is utilized extensively on spacecraft electronics is conformal coat. NASA-STD-8739.1 defines it as "a thin electrically nonconductive protective coating that conforms to the contours of the PWA." It consists typically of an epoxy with harder durometer (than other options) a coefficient of thermal expansion (CTE) that is similar to the substrate $\mathrm{PCB}$, high glass transition range and good dielectric properties. It's used on PCB's to both stake components, giving them structural support underneath and around their leads (see figure 16. Maxim 1239MEEE Package E16-4 Outline ${ }^{12}$ ) while also providing them with additional thermal conductivity to the PCB. For some unknown reason the same NASA-STD-8739.1 defines staking exclusively as "an electrically nonconductive adhesive material used for additional support after a part has been attached by mechanical or soldering process," omitting the thermal conductivity additions that 
staking provides. The conformal coat used on CP3 is a transparent polyurethane material called Arathane.

The same process that worked so efficiently for solder mask was performed again for conformal coat and generated the results in the table below. From the test results it appears that the conformal coat had little to no affect on the emissivity of the board. This was only done on locations where the coat was applied over solder mask. Note that for two of the measurements the emissivity did increase. It was postulated after the test that slightly angling the sensor head of the MI40 may have caused it to give an off reading. The emissivity of the conformal coat was shown to be identical to the material below.

Table 9. Emissivity Values for Conformal Coated Parts

\begin{tabular}{|c|c|c|}
\hline Run \# & Sample (Reference) Temperature & Emissivity (Final Gain Setting) \\
\hline 1 & 21.3 & .90 \\
\hline 2 & 21.3 & .92 \\
\hline 3 & 21.3 & .90 \\
\hline 4 & 21.3 & .90 \\
\hline 5 & 21.3 & .90 \\
\hline 6 & 21.3 & .91 \\
\hline
\end{tabular}

The results of this test could be misleading for other CubeSat teams however. Not all conformal coats are made alike. While the conformal coat used on CP3 is a transparent polyurethane material other conformal coat materials may be opaque or highly pigmented. Additionally, the conformal coat process is highly irregular and usually varies between both personnel and teams as to the end results thickness and variances across the board. Since there are so many 
inconsistencies to this material other teams using either a pigmented or opaque coat should independently test their coating for its radiative properties. As a note, previously Arathane material was named Uralane, and NASA documentation still refers to Uralane vice Arathane in most places.

\subsubsection{Improved Triple Junction (ITJ) and Ultra Triple Junction (UTJ) Solar Cells}

Spectrolab, the solar cell manufacturer and supplier for CP satellites, have extensively quantified the radiative properties for theirs solar cells and data is readily available on them. The figure below is extracted from their available specification sheets. While the CP3 satellite system uses the ITJ model the solar absorptance and emittance is the same for both cell types so future work using UTJ cells will also be covered by these calculations. Note that the $29.5 \% \mathrm{NeXt}$ Triple Junction (XTJ) Solar Cells do not conform to these values and have a lower absorptance value of .90 .

\section{Thermal Properties}

\begin{tabular}{l}
\hline Solar Absorptance $=0.92$ (Ceria Doped Microsheet) \\
\hline Emittance $($ Normal $)=0.85$ (Ceria Doped Microsheet)
\end{tabular}

Figure 29. Spectrolab ITJ and UTJ Cell Radiative Values ${ }^{16}$

The ITJ solar cells have an per cell of $27.3 \mathrm{~cm}^{2}$ and their tabs have an total effective area of $2.32 \mathrm{~cm}^{2}$ per panel. Thus $4.2 \%$ of the total area taken by the solar panels does not share the absorptance and emittance values provided above. Typically large satellite manufacturers or solar cell providers have 
advanced laying techniques that completely eliminate these extra tabs as an external viewable surface but CubeSat teams who purchase the cells at a reduced rate and populate the boards by hand currently do not modify the tabs so there is no loss of contact or lowered power throughput.

From Gilmore, the thin copper foil has an absorptance value of .30 and an emittance value of $.02 .^{2}$ When these values and the additional surface area are accounted for, the new average emittance value (calculated in Appendix D) is .81 and the absorptance value is .89 , for a net change of $4 \%$ and $3 \%$ respectively.

These changes are not large, but the point in effect is that a CubeSat engineer must often consider design parameters that even large spacecraft designers can negate or assume without repercussion. To be fair, the alternate is also very true. CubeSat designers have the luxury of shorter mission life, more benign LEO orbits and increasingly risk tolerant customers and/or missions than large spacecraft designers do. A decrease in the absorbed thermal energy by $5 \%$ is enveloped by conservatism and wide allowable temperatures for an engineer whose solar panels are isolated (e.g. Loral), use a spinning "thermal roll" design (e.g. Hughes) or have large tolerances (e.g. JPL/Northrop), but to a picosatellite which has none of the above, this is valuable data and can be used to more accurately predict the on-orbit environment. 


\subsection{Thermal Analyses and Models}

In this section several computations running the gamut of thermal analysis options are presented. First and foremost in the progression is the initial "back of the envelope" P's office analysis which spawned this whole idea. As this thesis is written in the spirit of CubeSats, the next portion is a review of the basics of spacecraft thermal analysis and their application to CubeSats so as to support other teams basic analysis needs. The last three sections carry the detailed thermal equilibrium calculations, heat generation by mode estimates, heat dispersion conduits and electrical "resistor" theory application, and finally the computer aided design (CAD) finite element and finite difference model generations and solutions.

\subsubsection{P's Office}

Because it could not be left out, the first calculation performed in 2006 which became the starting point for this thesis is attached, scanned in its original form in Appendix F. This calculation provided the idea that, considering an extremely simplified scenario, a picosatellite (specifically CP2 or CP3) could generate enough thermal energy to have a positive effect on their orbital temperature. The calculation utilized the equation 7 below in section 3.2.2, found in SMAD, to estimate a worst-case cold orbital equilibrium scenario with a one Watt averaged CP3 power use and heat generation. This resulted in a temperature of $254.4 \mathrm{~K}$. The second calculation utilized the same worst-case scenario but with an increased, albeit reasonable, heat generation of five Watts 
average for the satellite and resulted in a temperature of $273.3 \mathrm{~K}$. This difference in temperature (18.9K) was non-trivial and provided the first evidence that the $\mathrm{CP}$ bus, in an extremely simplified scenario, had the required heat generation to positively impact its thermal condition on orbit. As a note the equation we used neglects the background temperature of space (4K), which added conservatism to our cold-case scenario.

$$
Q_{\text {internal }}+Q_{\text {external }} A_{\text {satellite }}=\varepsilon_{\text {satellite }} \sigma A_{\text {satellite }}\left(T_{\text {Satellite }}^{4}\right)
$$

\section{Equation 7. SMAD Basic Satellite Thermal Balance Equation ${ }^{\mathrm{AC}}$}

\subsubsection{Satellite Thermal Analysis Overview}

This section provides a background and review of the key principles, properties and governing equations within satellite thermal analysis theory. It follows the general analysis review presented by Aerospace Corporation's "Spacecraft Thermal Control Handbook" and performed by Phillips and Gilmore 2 . Since the current generation of CubeSats do not use advanced heat pipes or pumped fluid loops the only two methods of heat transfer used in analysis for this thesis were conduction and radiation. Fourier's Law governing conduction is shown below in Equation 8. The heat flux between objects is $q^{\prime \prime}$, typically expressed in watts per meter squared $\left(\mathrm{W} / \mathrm{m}^{2}\right) .{ }^{17}$ The temperature gradient across the surface is $\frac{d T}{d x}$, typically expressed in terms of Kelvin per meter $(\mathrm{K} / \mathrm{m})$. Lastly $\mathrm{k}$ is the thermal conductivity of the material, typically expressed in terms of watts per meter degree Kelvin $\left(\mathrm{W} / \mathrm{m}^{\circ} \mathrm{K}\right)$. 


$$
q^{\prime \prime}=-k \frac{d T}{d x}
$$

\section{Equation 8. Fourier's Law}

Radiation is the sole method of energy addition and rejection for a satellite. The surface properties and geometry of a satellite determine the amount of energy a satellite takes in, reflects and emits. The amount of radiation absorbed by a surface is relative to a property called absorptivity, represented typically by the greek letter alpha, $a$. The amount of radiation emitted from a surface is relative to a property called emissivity, represented typically by the greek letter epsilon, $\varepsilon$. The amount of radiation transmitted through a material (surface) is relative to a property called transmissivity, represented by the greek letter tau, $\tau$. Lastly the amount of energy reflected by a surface is relative to a property called reflectivity, often represented by the greek letter lambda $\lambda .{ }^{18}$

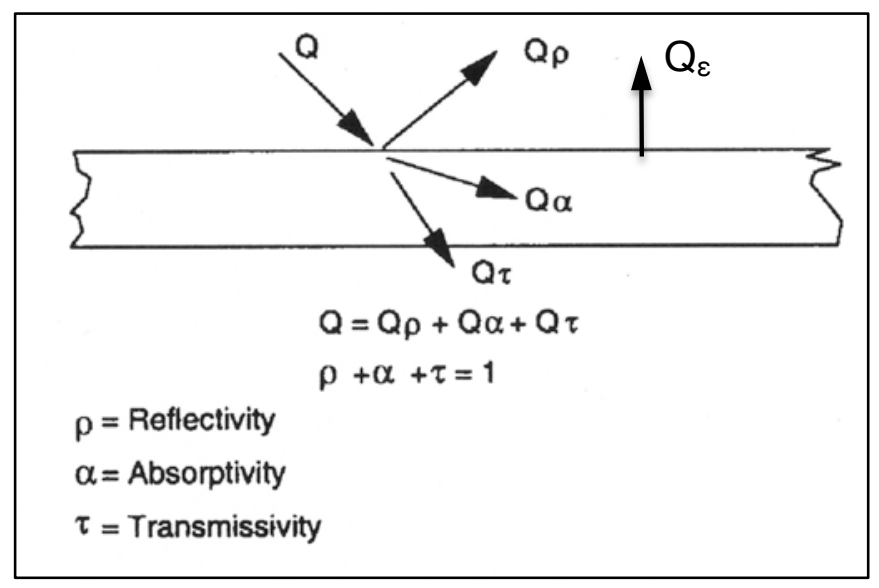

Figure 30. Incident Radiation on a Surface ${ }^{2}$

Figure 30 above clearly illustrates that of all the incident radiation $(Q)$, some is reflected $\left(Q_{p}\right)$, some is absorbed $\left(Q_{a)}\right.$ and becomes heat, and lastly some is 
transmitted through the body $\left(Q_{\tau}\right)$. In addition to these three, when the surface itself is warmer than absolute zero it will emit IR radiation $\left(Q_{\varepsilon}\right)$. For spacecraft purposes the two properties used most are absorptivity and emissivity with each being specially quantified as solar and infrared (IR), respectively. By categorizing them in this manner it reminds anyone looking up or applying the property that the specified absorptivity value applies to the solar spectrum while the emissivity property governs the IR portion of the spectrum. Some typical values for these properties are shown in the table below. Another key property listed in the table below (alpha/epsilon) is very useful to spacecraft thermal engineers. This material property gives a rough idea of whether the material will be a good emitter (low $a / \varepsilon$ ) or a good absorber (high $a / \varepsilon$ ). Put simply if a thermal engineer is choosing their surface materials to keep their satellite cool they'll choose a low $a / \varepsilon$ value material and if they need to keep their satellite warm they'll choose a high $a / \varepsilon$ value material. 
Table 10. Standard Spacecraft Emissivity, Absorptivity and Alpha/Epsilon Values ${ }^{19}$

\begin{tabular}{|l|r|r|r|}
\hline \multicolumn{1}{|c|}{ Material } & Absorbtivity $\alpha$ & Emissivity $\varepsilon$ & \multicolumn{1}{|c|}{$\alpha / \varepsilon$} \\
\hline Aluminium (6061-T6 alloy) as supplied & 0.379 & 0.0346 & 10.95 \\
\hline Aluminium (6061-T6 alloy) polished & 0.2 & 0.031 & 6.45 \\
\hline Aluminium with Alodine 1200S & & & \\
Chromate Conversion Coating & 0.08 & 0.15 & 0.53 \\
\hline Gold & 0.299 & 0.023 & 13.00 \\
\hline White epoxy paint & 0.248 & 0.924 & 0.27 \\
\hline Black paint & 0.975 & 0.874 & 1.12 \\
\hline Silver coated Teflon & 0.08 & 0.66 & 0.12 \\
\hline Aluminized Teflon (front surface) & 0.163 & 0.80 & 0.20 \\
\hline Aluminized 25 $\mu m$ Kapton (back surface) & 0.36 & 0.61 & 0.59 \\
\hline $\begin{array}{l}\text { Multi-Layer Insulation with aluminized } \\
\text { 25 } \mu \text { m Kapton cover sheet, large areas } \\
\text { without seams }\end{array}$ & & & \\
\hline Silicon solar cells (with cover glass) & 0.36 & $\mathrm{e}^{\star}=0.002$ & 180 \\
\hline $\begin{array}{l}\text { Gallium-Arsenide solar cells } \\
\text { (with cover glass) }\end{array}$ & 0.75 & 0.83 & 0.90 \\
\hline
\end{tabular}

The table above specifically makes no mention of several of the materials that are exposed on a CubeSat's external surfaces. This is because, as discussed in Section 2, typical spacecraft thermal designers don't have to work inside the confines of a picosatellite's volume, mass and budget. Their side panels are typically an aluminum honeycomb or composite layers with a carefully applied exterior surface finish. As CubeSats rely heavily on printed circuit boards and integrated circuits their external surfaces, outside of solar panel areas, are almost exclusively one of those two materials, neither of which is particularly well suited for additional thermal surface treatment (MLI excluded). This architecture of PCB panels with aluminum sub-structure is part of what enables satellites like CP2 and CP3 to have over $40 \%$ of their mass budget available to payload systems, altering it would mean potentially diminishing the payload mass and the 
entire justification for the mission (i.e. the payload). Since this design is critical to the entire architecture, the thermal design cannot drive a change to it but must instead find ways to work with it. Adaptive component usage is one way to work with this architecture. Figure 31 shows, for comparison and calculation purposes, the average surface area properties of the CP3 satellite. Refer to Appendix D for the detailed calculations used to generate an accurate value for the entire surface of the spacecraft.

\begin{tabular}{|lr|}
\hline SATELLITE SURFACE AVERAGED \\
Emissivity & 0.84 \\
Absorptivity & 0.87 \\
E/Alpha & 0.97 \\
\hline
\end{tabular}

Figure 31. Corrected Satellite Surface Property Averages (Satellite Whole) The corrected surface properties give $\mathrm{CP} 3$ an $\varepsilon / \alpha$ ratio of .97 . Using these surface properties above you must develop an energy balance equation showing all the radiant energy entering and exiting the system. The conservation of energy assures us that the sum of the absorbed energy and the emitted energy will come to a steady state over time. This assumes we are not converting any stored chemical energy into heat.

$$
Q_{\text {Absorbed }}-Q_{\text {Radiated }}=0
$$

\section{Equation 9. Energy Balance Equation}

Due to the satellites internal heat generation capabilities a $Q_{\text {Internal }}$ should be added generating the equation below. 


$$
Q_{\text {Absorbed }}-Q_{\text {Radiated }}+Q_{\text {Internal }}=0
$$

\section{Equation 10. Energy Balance Equation with Internal Heat Generation}

The governing equation for radiative heat flux to space is shown below where the symbol sigma $(\sigma)$ denotes the Stefan-Boltzman constant and is equal to $5.67 \times 10^{8}$ $\mathrm{W} / \mathrm{m}^{2} \mathrm{~K}^{4}$. The satellite temperature is denoted as $T_{\text {Satellite }}$, the temperature of space is denoted by $T_{\text {Space }}$ and the emissivity of the spacecraft is denoted with the symbol epsilon.

$$
Q_{\text {Radiation }}=\varepsilon_{\text {Satellite }} \sigma \mathrm{A}\left(T_{\text {Satellite }}^{4}-T_{\text {Space }}^{4}\right)
$$

\section{Equation 11. Radiative Heat Transfer (Emitted Heat)}

For satellites in Earth orbit the radiation flux can be broken into three components. The first component is solar flux and it is the largest potential energy source of the three. Even with the 11-year solar cycle the amount of energy radiating from the sun varies within a fraction of $1 \%$ at all times. ${ }^{2}$ Due to the eccentricity of its orbit, however, there is a slight alteration of the amount of incident solar energy reaching. The 'average' heating values used throughout this thesis are per Goddard Space Flight Centers Guideline GD-AP-2301 document. This is considered to be the NASA standard for Earth orbit environmental heating. At the summer solstice when Earth is farthest from the sun the intensity (shown below as $Q_{\text {sun }}$ ) is at its minimum of $1318 \mathrm{~W} / \mathrm{m}^{2}$ and during the winter solstice when the Earth is closest to the sun it is at its maximum of $1422 \mathrm{~W} / \mathrm{m}^{2}$. the average intensity value for solar flux is $1367.5 \mathrm{~W} / \mathrm{m}^{2}$ and its 
measured when the Earth is at its standard distance of $1 \mathrm{AU} .^{2}$ To calculate the solar flux being absorbed by a satellite use a derivation of equation 12 , shown below.

$$
Q_{\text {Solar }}=Q_{\text {sun }} A_{\text {Satellite }} \alpha_{\text {Satellite }}
$$

\section{Equation 12. Solar Flux Calculation}

For completeness the solar flux should also be adjusted for the angle of the spacecraft surface with respect to the sun. The amount of energy being absorbed by the surface area is a function of both its absorptivity and the angle psi $(\psi)$ between its surface normal and the sun (modeled as a point source).

$$
Q_{\text {Solar }}=Q_{\text {Sun }} A_{\text {Satellite }} \alpha_{\text {satellite }} \cos (\psi)
$$

\section{Equation 13. Solar Flux Adjusted for Spacecraft Angle}

The next component of radiative flux on orbit is Albedo. This is made up of solar energy being reflected off of a planet or moon, in the case of CubeSats this is Earth albedo. Unlike solar flux albedo can fluctuate significantly and is highly dependent on the location of the satellite. D. Gilmore notes in his book "Spacecraft Thermal Control Handbook" that variations in albedo make selection of the best albedo value for a certain thermal analysis rather uncertain and variations throughout the aerospace industry are common. ${ }^{2}$ Figure 32 below was produced by Lockheed Corporation to serve as an estimating tool for Earth albedo. 




Figure 32. Solar Albedo Calculation Chart ${ }^{2}$

To determine the albedo value from the calculation chart one must first establish their orbital properties and then using their beta angle and the reference surface geometry an approximate value for albedo can be obtained. Another method for establishing the albedo value is shown in Equation $\mathrm{X}$ below. Here the user must input the albedo percentage, which from NASA guidelines varies from .25 to .35 . This method is only suggested for developing maximum and minimum case values (using .30 and .23 respectively). $Q_{\text {sun }}$ is the chosen solar heat flux, $a$ is the chosen albedo percentage, $r_{\mathrm{e}}$ is the radius of the Earth. 


$$
Q_{\text {Albedo }}=Q_{\text {Sun }} \alpha K\left[\frac{r_{e}^{2}}{\left(r_{e}+h\right)^{2}}\right] A_{\text {Satellite }}
$$

\section{Equation 14. Albedo Heat Load}

$\mathrm{K}$ is defined as the reflected solar energy factor, which accounts for the reflection properties as the albedo goes through the atmosphere and is defined separately below.

$$
K=.664+.521-.203\left(\frac{r_{e}}{r_{e+}+h}\right)^{2}
$$

\section{Equation 15. Reflected Solar Energy Factor ${ }^{19}$}

Earthshine is the long wave IR radiation that every location on the planet produces. It allows the entire planet to maintain its own thermal stability. For Low Earth Orbits (LEO) it can be a significant heating factor and when in eclipse it becomes the sole external heating factor available to the satellite. In Equation $\mathrm{X}$ below, $\mathrm{Q}_{\mathbb{R}}$ is the total flux of long wave IR from the Earth.

$$
Q_{I R}=Q_{\text {Earth IR }}\left(\frac{r_{e}^{2}}{\left(r_{e}+h\right)^{2}}\right) \varepsilon A_{\text {Satellite }}
$$

\section{Equation 16. Earthshine IR Radiation}




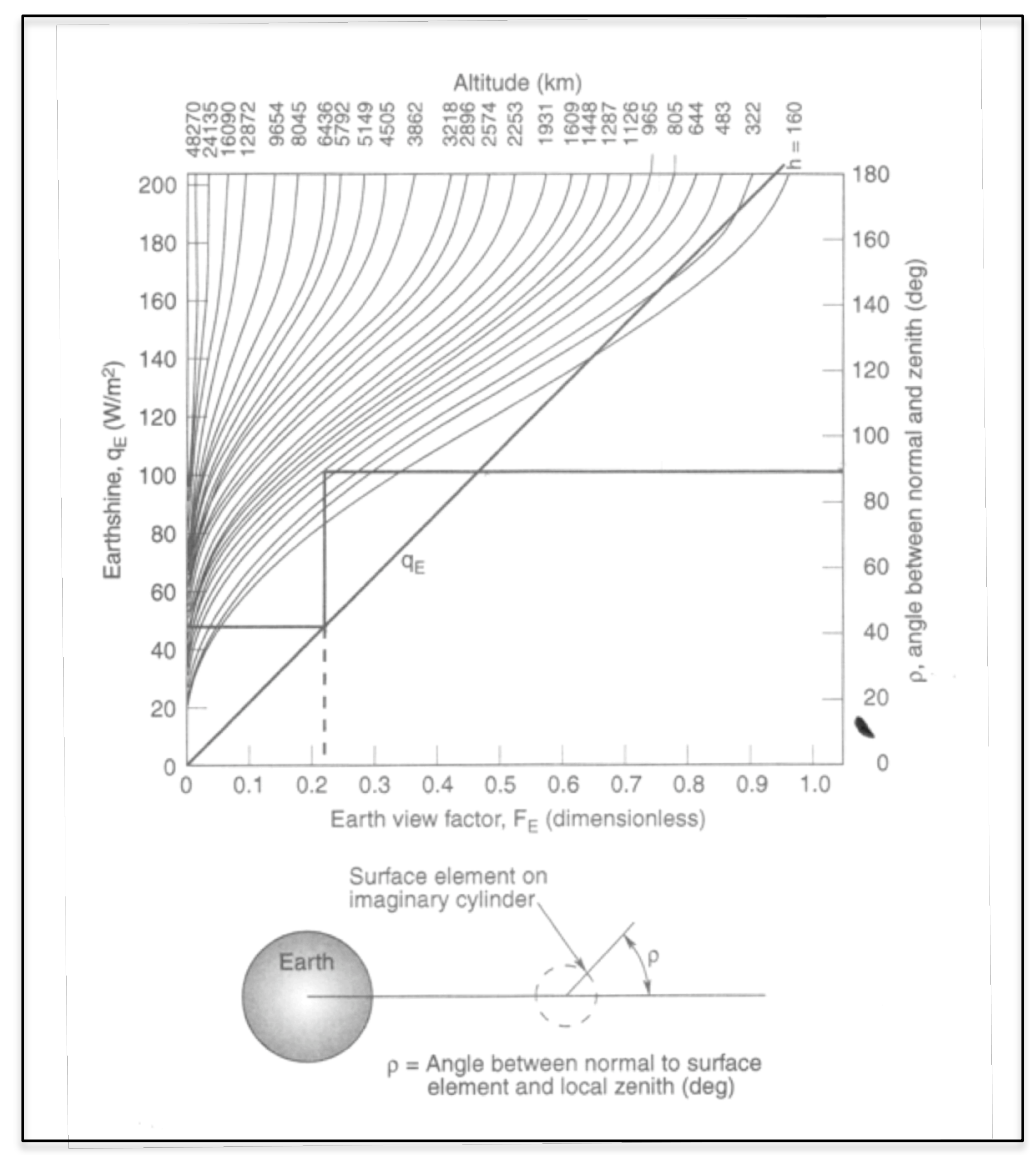

Figure 33. Earthshine IR Calculation Chart $^{2}$

Another method for establishing Earthshine that is utilized by the Lockheed Martin Company is a calculation chart shown in figure 33 (above). Data points can be interpolated and a maximum and minimum can be established in that manner as well. An interesting feature of Earthshine is its variability. According to data collected by NASA on the Earth Radiation Budget Experiment (ERBE) its value ranges from $108 \mathrm{~W} / \mathrm{m}^{2}$ to $300 \mathrm{~W} / \mathrm{m}^{2}$ depending on the orbital and ground conditions. See the figure 34 below illustrating the wide variability for a LEO satellite. 


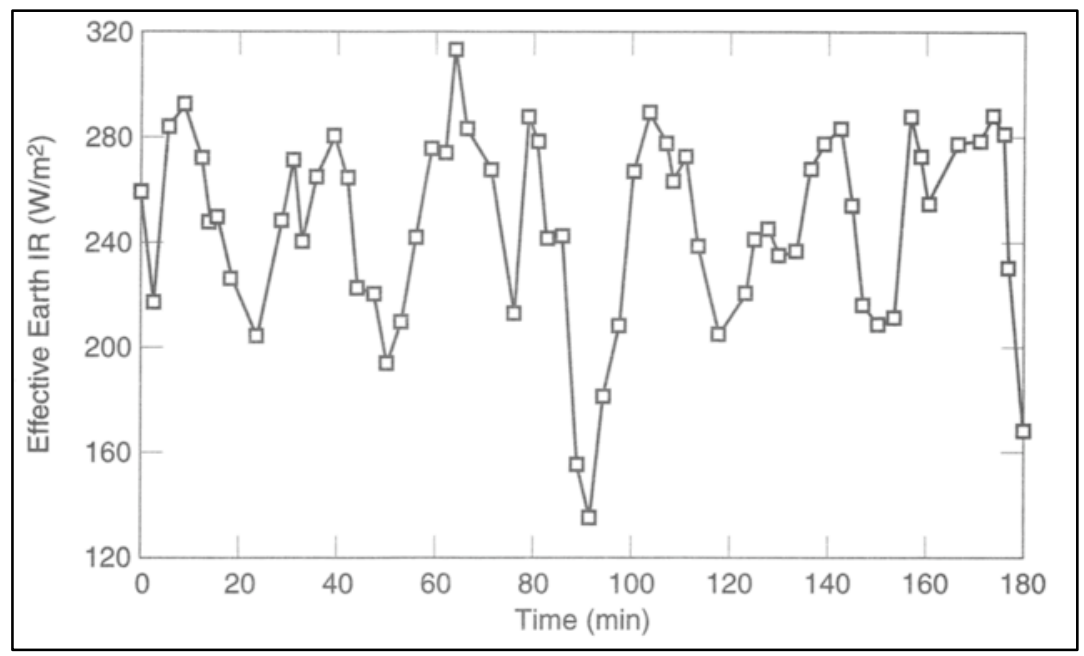

Figure 34. Earthshine IR for a LEO Spacecraft (Sample) ${ }^{2}$

The combination of the three listed radiation sources vary throughout the orbit of a satellite and at some points all three generate a maximum heating condition and at other points only IR exists and the heating condition is minimized. The Figure 35 below illustrates the combination of the sources along with some nominal values.

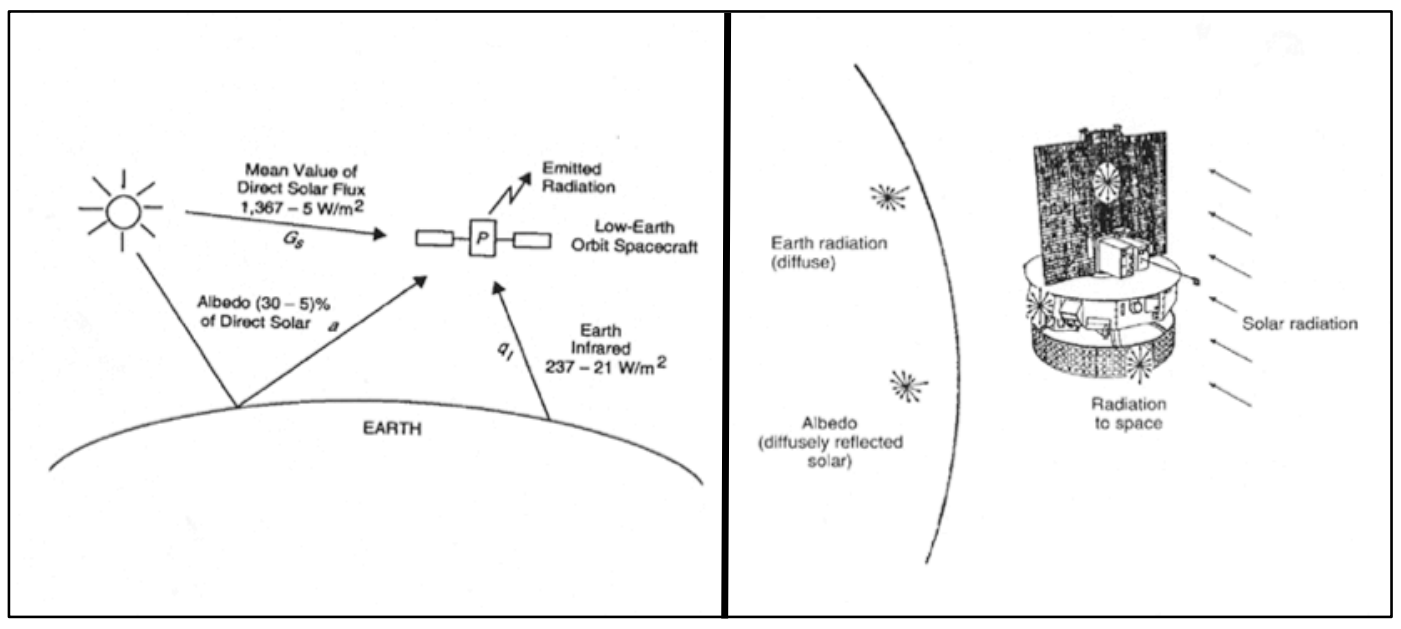

Figure 35. Combined On-Orbit Heat Loads ${ }^{2}$ 
The minimum and maximum conditions are shown in figures 36 and 37 , illustrating how the CubeSat is eclipsed by the Earth, creating a minimum heating condition, and then comes out of eclipse and receives the maximum amount of radiative heat flux.

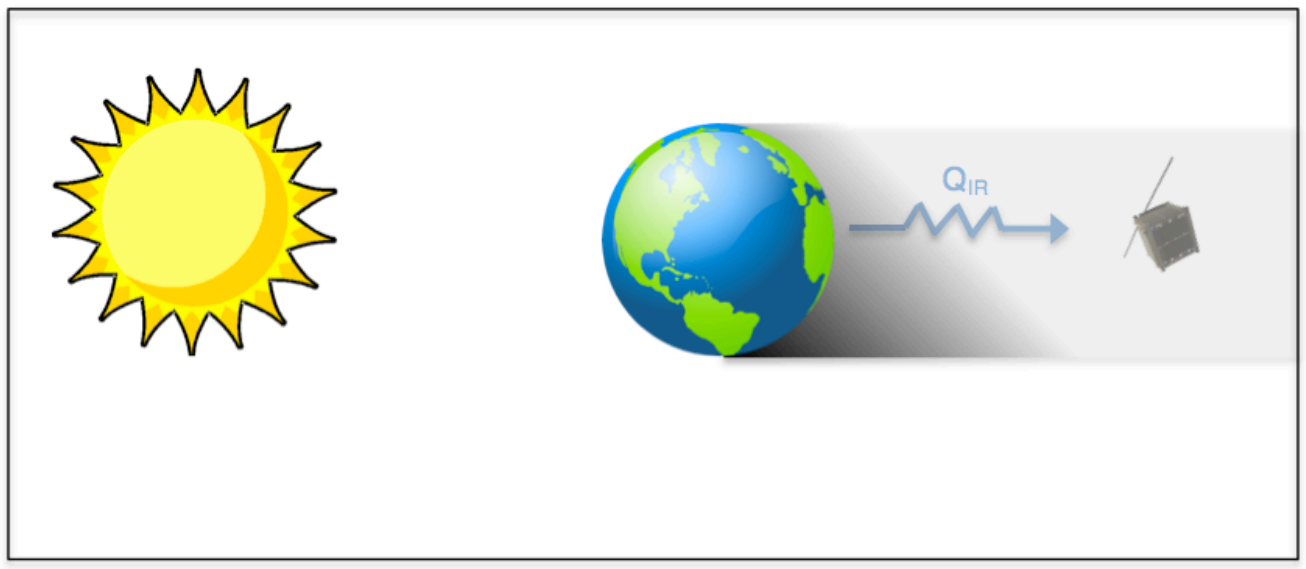

Figure 36. Worst Case Cold Condition

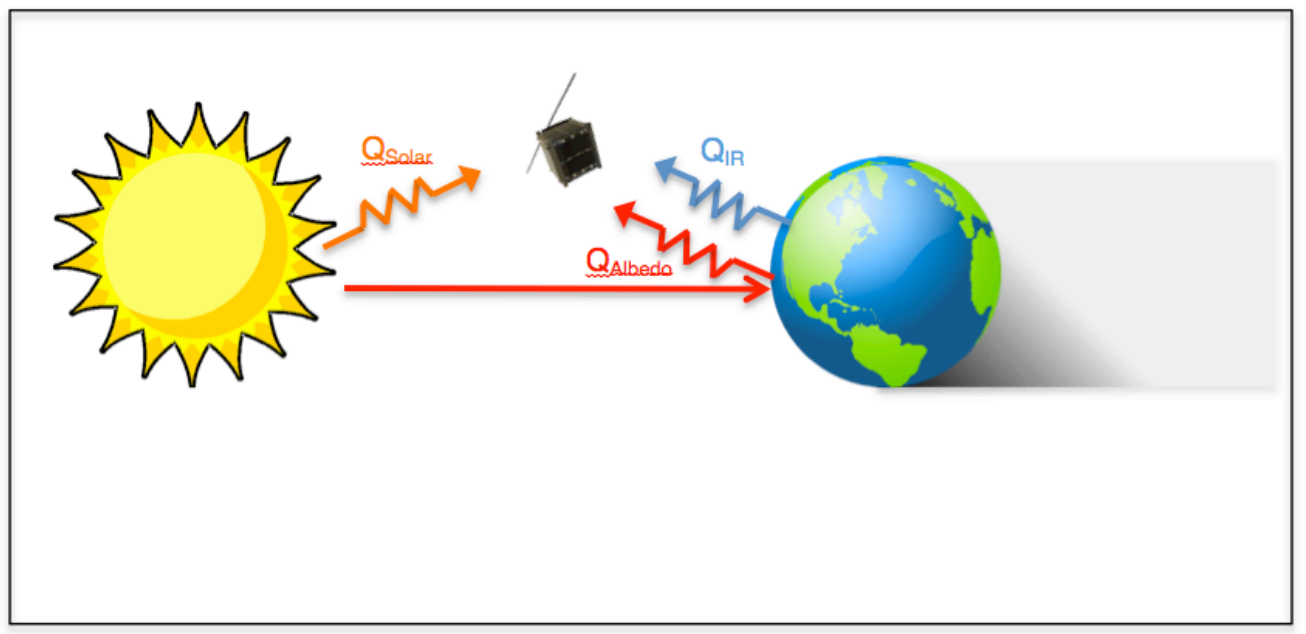

Figure 37. Worst Case Hot Condition

For CP2 and CP3 the Albedo loads are appropriate to model as a collimated source simply because the altitude in question is above the point where Albedo is 
affected by orientation and shading. For most spacecraft a general assumption for albedo view factor is 0.3 with a cosine reduction (as discussed in the analytical baseline section 3.3.3) in the reflected energy from the subsolar point of the orbit to the terminator.

\subsubsection{General Thermal Equilibrium Calculation (Analytical Baseline)}

To formalize the back of the envelope analysis and provide the necessary information for other CubeSat teams to calculate their own accurate equilibrium hot and cold temperatures the steps leading to the radiative loading are detailed in this section. The hottest orientation, as shown in Figure 37 in section 3.3.2, results when all three radiative heat fluxes are being applied on the largest area of the satellite. Note that the Albedo load factor used is well above the Goddard guideline; this is because those limits are to be used only for average orbital equilibrium calculations and not for maximum loading conditions. With our orbit inclination being greater than 90 degrees, the following worst-case hot heat load assumptions can be made:

- Earth IR is 244 Watts $/ \mathrm{m}^{2}$

- Solar Albedo is 57 percent

- Solar Flux is $1414 \mathrm{~W} / \mathrm{m}^{2}$

The equilibrium state calculations rely upon the surface area maximum and minimum values for the viewable cross-section of the satellite, which changes, on orbit with respect to attitude. A calculation to determine the cross sectional area 
was performed and is included in Appendix G. The resulting graphic below of maximum incident area $\left(.01732 \mathrm{~m}^{2}\right)$ was generated from this calculation.

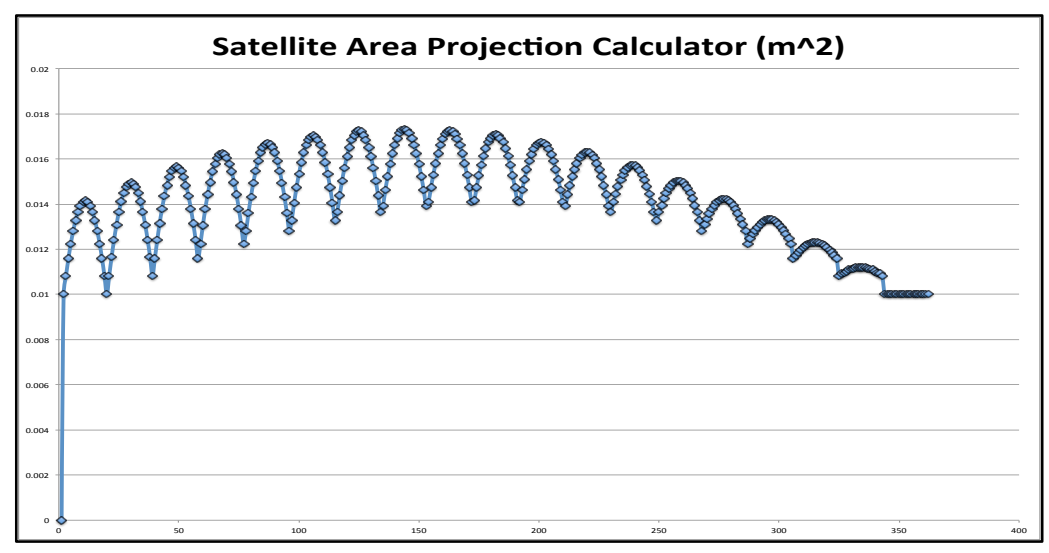

Figure 38. Satellite Area Projection Calculator - Surface Area vs. Attitude

Maximum area is not a reasonable assumption for picosatellites like CP2 and CP3 however because they do have some amount of roll, pitch and yaw while on orbit. Taking an average of the projected area is far more realistic and gives a result of $.0149 \mathrm{~m}^{2}$. This also lowers the affect of the reflectivity on the exposed surfaces to a point where it can be neglected for our maximum and minimum calculations.

Using the inputs derived for the hot case generated the values in the table below (supporting calculations and tables shown in Appendix $\mathrm{H}$ ). Adjusting to account for the true surface emissivity and absorptivity of the CubeSat being evaluated (CP3) resulted in a 10 degrees change to the worst-case hot values of slightly over (12\% difference). It was also interesting to note that Gilmore's suggestion of limiting Earthshine to only one face generated a significantly lower worst-case hot situation. 
Table 11. Hot Case Equilibrium Calculation Results

\begin{tabular}{|l|c|c|}
\hline & Solved for T Kelvin & (Celsius) \\
\hline CASE 4 - Hot w/o Adjustments & 356 & 83 \\
\hline CASE 5 - Hot w/ Adjustments & 346 & 73 \\
\hline CASE 6 - Hot w/ Single Face (Gilmore) & 315 & 42 \\
\hline
\end{tabular}

After reviewing these calculation results against on-orbit measurements, the Gilmore result (while not conservative) was found to be far more accurate a prediction (for hot case conditions). Actual on-orbit measurements established during the operation of CP3 peaked around 40 degrees Celsius (see figure 39).

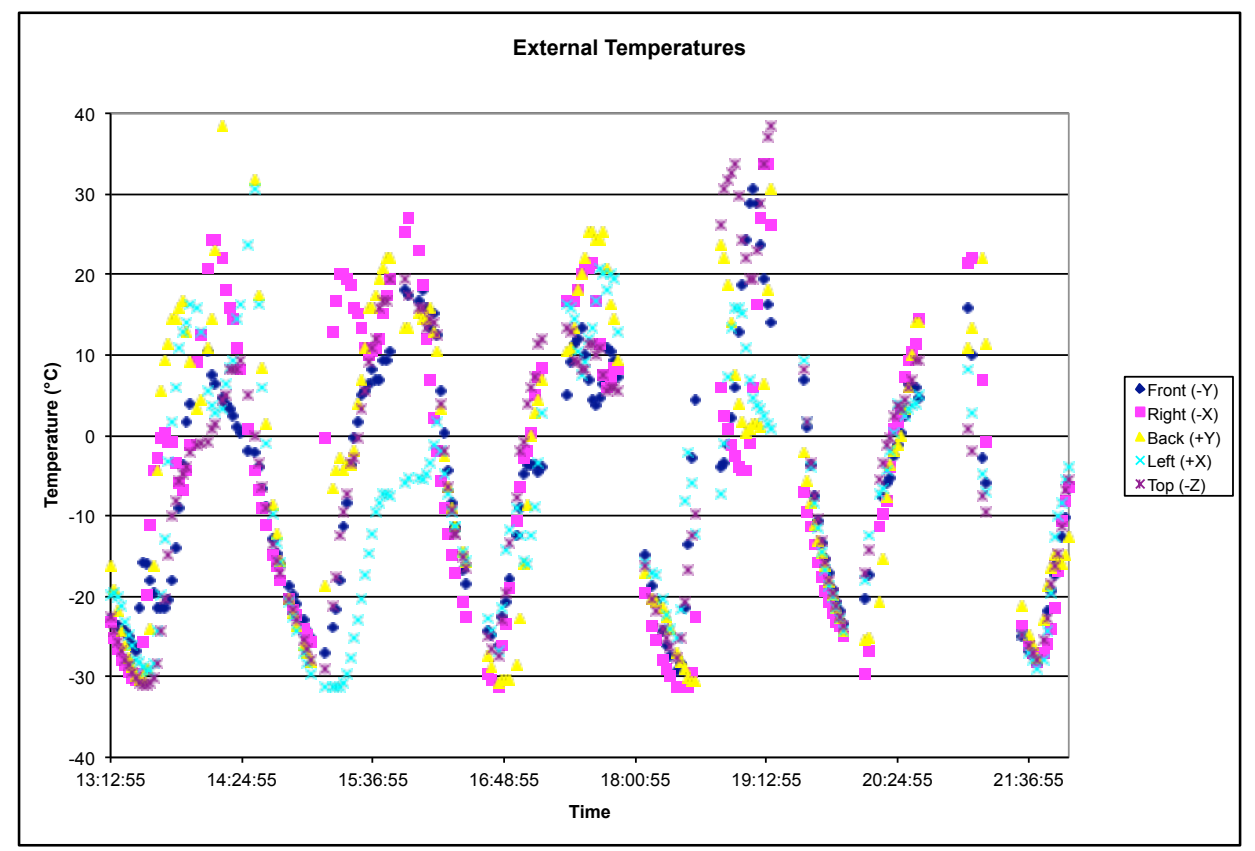

Figure 39. External On-Orbit Temperature Maximums (Externals)

The coldest orientation, as shown in figure 36 in section 3.3.2, occurs when the minimum Earth IR is being applied across the smallest cross sectional area of the satellite. With an orbit inclination of greater than 90 degrees the following worst-case cold heat load assumptions can be made: 
- Earth IR is 218 Watts $/ \mathrm{m}^{2}$

- Solar Albedo is 0 percent (eclipse)

- Solar Flux is $0 \mathrm{~W} / \mathrm{m}^{2} \quad$ (eclipse)

Using these assumptions and a worst-case area projection for the cold case was calculated and is shown in the following table (supporting calculations shown in Appendix $\mathrm{H}$ ). The table also shows a best-case IR assumption that J. A. Wiebelt suggests in his book. ${ }^{18}$ This assumes that Earth IR is a diffuse property due to the longer wavelength bending in the atmosphere and should be applied on all viewable surfaces. This obviously generates a more favorable cold case temperature of $-32^{\circ} \mathrm{C}$.

Table 12. Cold Case Equilibrium Case Results

\begin{tabular}{|l|c|c|}
\hline & Solved for T Kelvin & (Celsius) \\
\hline CASE 2a - Cold w/o Adjustments & 260 & -13 \\
\hline CASE 2b - Cold w/ Adjustments & 241 & -32 \\
\hline CASE 2c - Cold w/ Single Face (Gilmore) & 205 & -68 \\
\hline
\end{tabular}

The the cold case condition, using Gilmore's method, was found to be, as expected, far more conservative at $-68^{\circ} \mathrm{C}$ than the other methods, which account for diffuse IR. The adjustments made based on the detailed measurement and calculations of the true surface emissivity and absorptivity of the CubeSat being evaluated (CP3) resulted in a significant change to the worst-case cold values of almost $20^{\circ}$ lower (146\% lower). As you can see in figure 40 the actual on-orbit worst-case (external-thermistor) cold temperatures $\left(-33^{\circ} \mathrm{C}\right)$ lined up quite well with the adjusted calculations. The actual mass of the spacecraft on orbit is not 
at $-33^{\circ} \mathrm{C}$ but the external thermistors and surfaces react quickly (shock) to the eclipse environment.

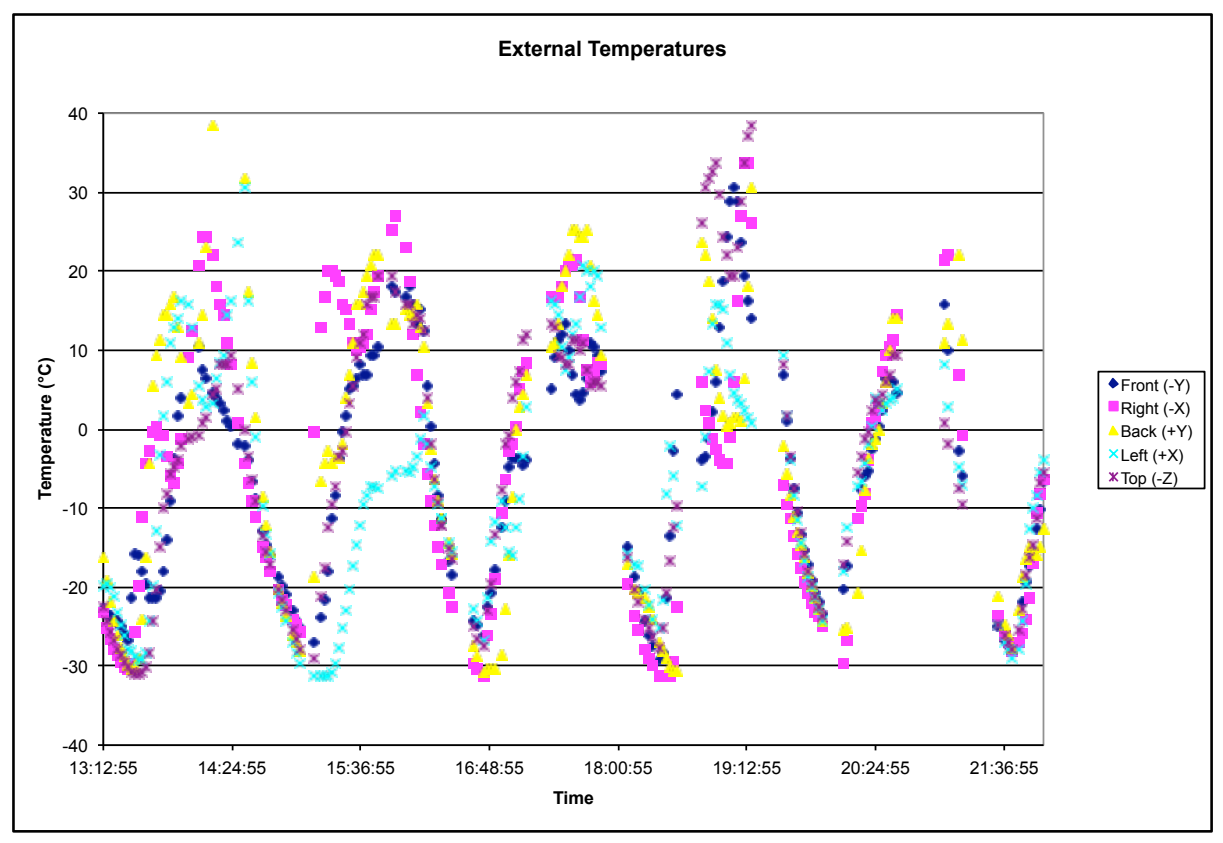

Figure 40. External On-Orbit Temperature Maximums (Externals)

This differential in cold case calculated temperature was one of the most substantial pieces of evidence that validated the importance of a CubeSat designer accurately evaluating, measuring and calculating their system's surface properties.

To move beyond the equilibrium worst-case evaluations the basic orbital parameters for the satellite in question need to be established so that beta angle, eclipse period and other key thermal parameters change over time could be deduced. This began with calculating the period, or time required to make one orbit about the Earth, and used the following equation. 


$$
P=2 \pi\left(\frac{a^{3}}{\mu}\right)^{.5}
$$

\section{Equation 17. Orbital Period}

In the orbital period equation $\mathrm{P}$ is the period in seconds, $\mu$ is the product of the universal gravitational constant and the mass of the planet where for LEO satellites $\mu$ is equal to $3.986 \times 10^{14} \mathrm{~m}^{3} / \mathrm{s}^{2}$ and a is the semimajor axis of the orbit, the average of the radius of perigee and apogee. For our CP3 orbit we had a semimajor axis of $7096 \mathrm{~km}$ leading to an (circular assumption) orbital period of 5949 seconds or 99 minutes. One of the most useful orbital parameters from a thermal standpoint is the beta angle $\beta$. It helps in visualizing the orbital thermal environment for LEO satellites. ${ }^{2}$ It is defined to be the minimum angle from the orbital plane and the solar vector with a value between -90 and +90 degrees. The equation for beta angle is shown below.

$$
\begin{aligned}
& \beta=\sin ^{-1}\left(\cos \left(\delta_{S}\right) \sin (R I) \sin \left(\Omega-\Omega_{S}\right)+\sin \left(\delta_{S}\right) \cos (R I)\right) \\
& \text { Equation 18. Calculating Beta Angle Given Ascending Nodes }
\end{aligned}
$$

\section{Equation 18. Calculating Beta Angle Given Ascending Nodes}

In this equation $\delta_{S}$ is the declination of the sun, RI is the orbit inclination, $\Omega_{S}$ is the right ascension of the sun, and $\Omega$ is the right ascension of the ascending node. ${ }^{2}$ Given that the DNEPR launch was at local 2200 the beta angle was found to be approximately 44 degrees which, using the equation below, generated an eclipse fraction of .33. 


$$
f_{E}=\frac{1}{180} \cos ^{-1}\left[\frac{\left(h^{2}+2 R h\right)^{.5}}{(R+h) \cos (B)}\right]
$$

\section{Equation 19. Eclipse Fraction by Beta Angle and Altitude}

Using STK the beta angle was calculated and plotted in Figure 41 below (starting angle is denoted with the red marker). The variance in beta angle causes variances in the lighting conditions present on the satellite and the resulting eclipse lengths.

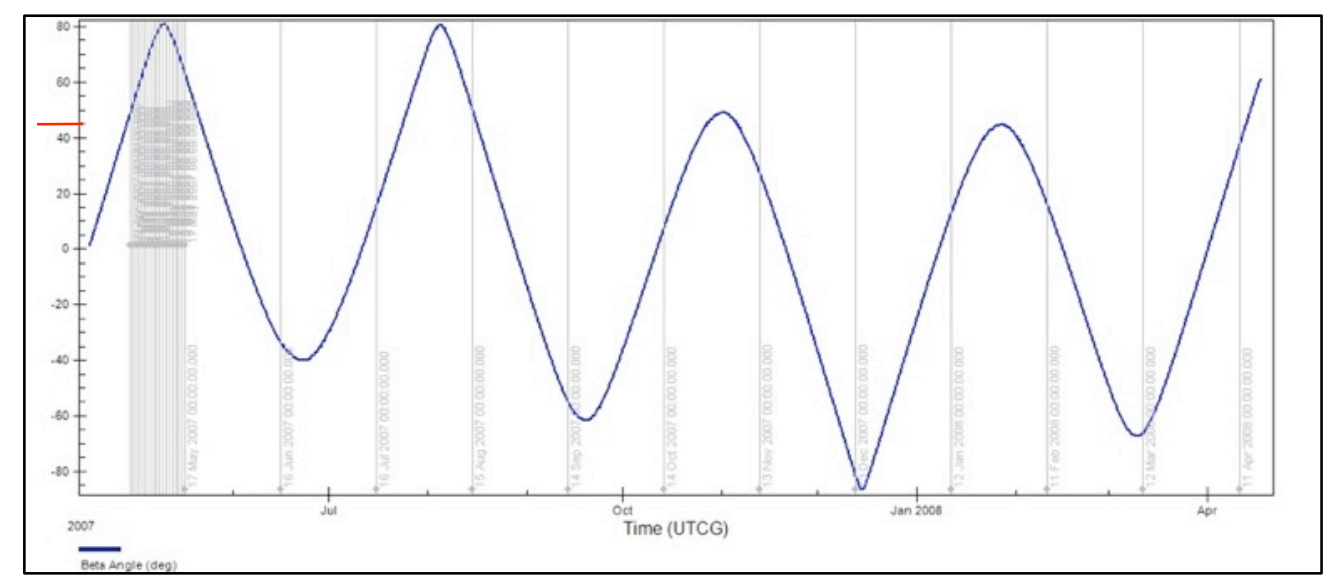

Figure 41. Beta Angle Plot (v. Time)

The eclipse and lighting conditions present on the satellite during its first twelve hours on orbit are shown below in Figure 42. The umbra (eclipse) and penumbra (partial eclipse) are clearly about 30 minutes with a precise value of 32.98 minutes. One of the key parts of this graph is to illustrate the speed at which CP3 moves from complete eclipse into full sun. Known as thermal shock this situation takes the satellite from its coldest condition seeing minimal Earthshine and the cold of deep space into full solar irradiance and full albedo reflection as well. 


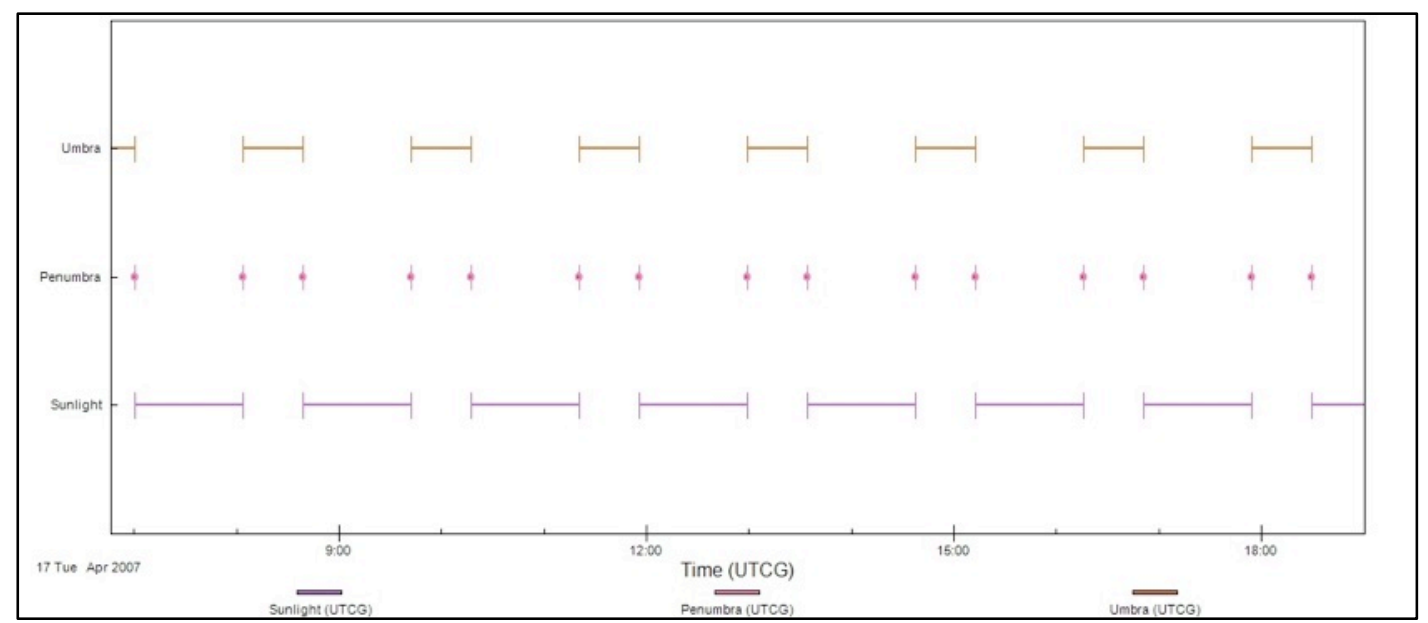

Figure 42. CP3 Lighting Conditions (12 Hour Plot)

With an established eclipse fraction and orbital period a concise table was built to track the values for use in radiative loading iterations and analyses. The table below shows the general orbital properties and the sunlit orbit fraction.

Table 13. CP3 Calculated Orbital Properties

\begin{tabular}{|l|r|l|}
\hline \multicolumn{1}{|c|}{ Orbit Properties: } \\
\hline Semimajor Axis: & 7105.20 & $\mathrm{~km}$ \\
\hline Eccentricity: & 0.010 & \\
\hline Inclination: & 98.00 & $\mathrm{deg}$. \\
\hline Period: & 99.330 & minutes \\
\hline Sunlit Orbit Fraction: & 0.67 & \\
\hline
\end{tabular}

\subsubsection{Heat Generation by Mode}

Satellites generate heat while running their electronics and the only difference with CubeSats is there is obviously less power available to generate heat due to their size and solar panel limitations. One of the greatest power draws on the CP bus is a COTS amplifier (RFMD RF2117) designed for use with 
RF signals between 400 and $500 \mathrm{MHz}$. The part, as implemented on the CP bus, operates at a supply voltage of $3 \mathrm{~V}$ and sinks a maximum of $1,100 \mathrm{~mA}$ of current, thus the $3 \mathrm{~V}$ operation consumes approximately $3.3 \mathrm{~W} .{ }^{20}$

One of the other heavy power draws on the system is the Attitude Determination and Control Subsystem (ADCS). It pulls approximately 1,000 mA off the battery directly using 3.7-4.0 $\mathrm{W}$ total. The ADCS also has a specific command feature allowing the power draw, duty factor and duty cycle to be set independent of one another. Beyond the communications system's beacon settings there are no other subsystems that have a unique command scheme like this. These two (ADCS \& C\&DH) subsystems make up almost three quarters of the total energy being used during the (peak) operation of the satellite. Logically with such large power draws these were also the prime subsystems for attempting to provide an on-orbit heat supply. The total power draw for each subsystem is shown in Table 14 below.

Table 14. Heat Generation by Component/System (CP3)

\begin{tabular}{|l|r|r|r|r|r|}
\hline & \multicolumn{1}{|c|}{ Current: } & \multicolumn{1}{|c|}{ Current: } & Prbit Average & Peak Sub-system & Average Sub-system \\
\hline Component: & & Revits: & Power: \\
Command Data Handling: & $0 \mathrm{amps}$ & $0.026 \mathrm{amps}$ & 1 & 0.078 watts & 0.0858 watts \\
Communications: & $0 \mathrm{amps}$ & $0.024 \mathrm{amps}$ & 1 & 0.072 watts & 0.0792 watts \\
Transmitter: & $1 \mathrm{amps}$ & $0 \mathrm{amps}$ & 0.1 & 3.7 watts & 0.37 watts \\
Side Panels: & $0 \mathrm{amps}$ & $0.035 \mathrm{amps}$ & 1 & 0.105 watts & 0.1155 watts \\
Attutude Control System: & $1 \mathrm{amps}$ & $0 \mathrm{amps}$ & 0.01 & 3.7 watts & 0.037 watts \\
Payload: & $0.25 \mathrm{amps}$ & $0 \mathrm{amps}$ & 0.3 & 0.925 watts & 0.2775 watts \\
TOTAL & $2.25 \mathrm{amps}$ & 0.085 amps & & & \\
Total Output Power/Energy Per Regulator: & 9.45 watts & 0.255 watts & & 8.58 watts & 0.965 watts \\
Total Input Power/Energy Per Regulator: & 10.0531915 watts & 0.30141844 watts & & 10.35460993 watts & 1.16459191 watts \\
\hline
\end{tabular}

Using the power draws by subsystem above and implementing them into command schemas with associated duty factor adjustments for testing and verification generated the following table 15. 
Table 15. Heat Generation by Mode

\begin{tabular}{|c|c|c|c|c|c|c|c|}
\hline & C\&DH & COMM & $\mathrm{BF}$ & Side Panels| & ADCS & Pavload & TOTAL Draw (W) \\
\hline \multicolumn{8}{|l|}{ Mode 1} \\
\hline Standard Operations & 0.0858 & 0.0792 & 0.3700 & 0.1155 & 0.0370 & 0.2775 & 1.062 \\
\hline \multicolumn{8}{|l|}{ Mode 2} \\
\hline a. Increased Beacon Rate (90 sec) & 0.0858 & 0.0792 & 0.5740 & 0.1155 & 0.0370 & 0.2775 & 1.286 \\
\hline b. Increased Beacon Rate $(60 \mathrm{sec})$ & 0.0910 & 0.0792 & 0.9600 & 0.1155 & 0.0370 & 0.2775 & 1.716 \\
\hline \multicolumn{8}{|l|}{ Mode 3} \\
\hline Mission Payload ENABLED (On) & 0.0910 & 0.0792 & 0.3700 & 0.1155 & 0.0370 & 0.9250 & 1.779 \\
\hline \multicolumn{8}{|l|}{ Mode 4} \\
\hline a. ADC On & 0.0910 & 0.0792 & 0.3700 & 0.1155 & 3.7000 & 0.2775 & 5.097 \\
\hline b. ADC On \& Increased Beacon $(90 \mathrm{sec})$ & 0.0910 & 0.0792 & 0.5740 & 0.1155 & 3.7000 & 0.2775 & 5.321 \\
\hline C. ADC On \& Increased Beacon $(60 \mathrm{sec})$ & 0.0910 & 0.0792 & 0.9600 & 0.1155 & 3.7000 & 0.2775 & 5.746 \\
\hline \multicolumn{8}{|l|}{ Mode 5} \\
\hline Increased "Data Drop" Contact (+.34DF) & 0.0910 & 0.0792 & 2.4790 & 0.1155 & 0.0370 & 0.2775 & 3.387 \\
\hline
\end{tabular}

The heat input by each mode was calculated above to support the FD/FEA simulations in section 3.3.5 and the analytical hot and cold case presented in table 11 and table 12 in section 3.3.3. These were based on data from previous testing on both $\mathrm{CP} 2$ and $\mathrm{CP} 3$. Post physical testing the PPI and SPI downloaded were used to confirm actual power draw. As a note this is not an accurate or precise enough method to confirm anything below .3W/hr but was the best available bus power metric, and was considered acceptable for these purposes. If more electrical engineers were available, and if time allowed, detailed measurements both pre and post-testing of the battery voltage along with posttest battery charge timelines could be measured to increase the accuracy of these calculations.

The potential heating modes were evaluated analytically using a steady state average based on the actual power usage cycle (table 15. Heat Generation 
by Mode). Using the equations and methodology presented in 3.3.3 the different satellite modes produced the following Cold Case by Mode, table 16 below.

Table 16. Cold Case by Mode

\begin{tabular}{|l|c|c|}
\hline & Solved for T Kelvin & (Celsius) \\
\hline CASE 3 - Mode 1 (Standard Operations) & 241 & -32 \\
\hline CASE 3 - Mode 2a. (Inc. Beacon Rate - 90s) & 253 & -20 \\
\hline CASE 3 - Mode 2b. (Inc. Beacon Rate - 60s) & 258 & -15 \\
\hline CASE 3 - Mode 3. (Payload Enabled - ON) & 258 & -15 \\
\hline CASE 3 - Mode 4a. (ADC ON) & 287 & 14 \\
\hline CASE 3 - Mode 4b. (ADC ON \& Beacon Inc. - 90s) & 289 & 16 \\
\hline CASE 3 - Mode 4c. (ADC ON \& Beacon Inc. - 60s) & 292 & 19 \\
\hline
\end{tabular}

From the results obtained by evaluating the adjusted baseline cold case against an identical exterior condition but with the supplementary $Q_{\text {Internal }}$ heat flux being supplied by each of the modes it became evident that the initial evaluation held true. This took into consideration all of the detailed surface properties along with a comprehensive internal heat generation calculation. The results for each mode were reviewed against verification criteria 1.1, repeated below for reference and calculated in table 17 .

1. The thermal condition of the satellite shall be more* stable with the implementation of the thesis than via passive control and standard on-orbit operations.

1.1. *A $15 \%$ reduction in spacecraft subsystem component temperature variability across maximum and minimum orbital heat flux conditions 
Table 17. Criteria 1.1 Verification Evidence; Percent Reduction Maximum to Minimum

\begin{tabular}{|l|c|c|c|}
\hline Verification Criteria 1.1 - 15\% Orbital Max-Min Reduction & Solved for T Kelvin & (Celsius) & $\%$ Reduction \\
\hline CASE 2b - Cold w/ Adjustments & 241 & -32 & \\
\hline CASE 5 - Hot w/ Adjustments & 346 & 73 & \\
\hline CASE 3 - Mode 1 (Standard Operations) & 241 & -32 & $0.0 \%$ \\
\hline CASE 3 - Mode 2a. (Inc. Beacon Rate - 90s) & 253 & -20 & $11.3 \%$ \\
\hline CASE 3 - Mode 2b. (Inc. Beacon Rate - 60s) & 258 & -15 & $15.6 \%$ \\
\hline CASE 3 - Mode 3. (Payload Enabled - ON) & 258 & -15 & $16.2 \%$ \\
\hline CASE 3 - Mode 4a. (ADC ON) & 287 & 14 & $43.4 \%$ \\
\hline CASE 3 - Mode 4b. (ADC ON \& Beacon Inc. - 90s) & 289 & 16 & $45.0 \%$ \\
\hline CASE 3 - Mode 4c. (ADC ON \& Beacon Inc. - 60s) & 292 & 19 & $47.9 \%$ \\
\hline CASE 3 - Mode 5. (Increased "Data Drop") & 273 & 0 & $30.5 \%$ \\
\hline
\end{tabular}

As can be seen in table 17, the verification criterion that called for a reduction of at least $15 \%$ in the variability across minimum and maximum conditions was met by modes $2 b, 3,4 a, 4 b$ and 5 . Mode $2 a$ had an $11 \%$ reduction that shows a significant improvement but did not quite meet the established criterion. Since equilibrium calculations negate transient effects on orbit this mode still could be verified through thermal vacuum testing and on-orbit tests but modes $3,4 a, 4 b$ and 5 obviously meet the verification criterion with margin.

These conditions and heating values assume we are maintaining a constant maximum temperature consistent with our standard operations mode. This assumption has a different level of applicability for each set of modes. Based on the ability to set modes 3 and 4 with distinct expiration timelines, this assumption is inherently true. Mode 5 requires no assumption since it already maintains that positive contact must be made and if no command is sent during maximum heat conditions it will not continue; therefore it is a true assumption. Modes $2 \mathrm{a}, 2 \mathrm{~b}, 4 \mathrm{~b}$ and $4 \mathrm{c}$ require users to enable/disable them. Assuming that a user can disable the mode prior to coming out of eclipse is not always realistic, 
especially if contact or acceptable passes are limited. However modes $4 \mathrm{a}$ and $4 b$ inherently will degrade to modes $2 a$ and $2 b$ (ADCS "OFF") and as such will not drive a significant increase in worst-case hot temperatures (see Appendix $\mathrm{H}$ for "Worst-Case Hot Mode Adjusted").

For a secondary verification, using worst-case conditions, the SEET tool in STK was used to model a single-node CP3 with the mass, surface properties and then placed into the appropriate DNEPR orbit $(792 \mathrm{~km} \times 645 \mathrm{~km})$ and allowed to propagate over several orbits. The same simulation was performed for several internal heating conditions (noted in each figure). The conditions that SEET uses as a standard are significantly lower in incident energy than those recommended by both Wertz and Gilmore and as such come in with lower temperatures than were calculated using energy balance thermal equilibrium equations (even using Gilmore's single face equilibrium equation). In the SEET simulations of CP3's the standard operations mode the satellite mass varied between $-74^{\circ} \mathrm{C}$ and $33^{\circ} \mathrm{C}$.

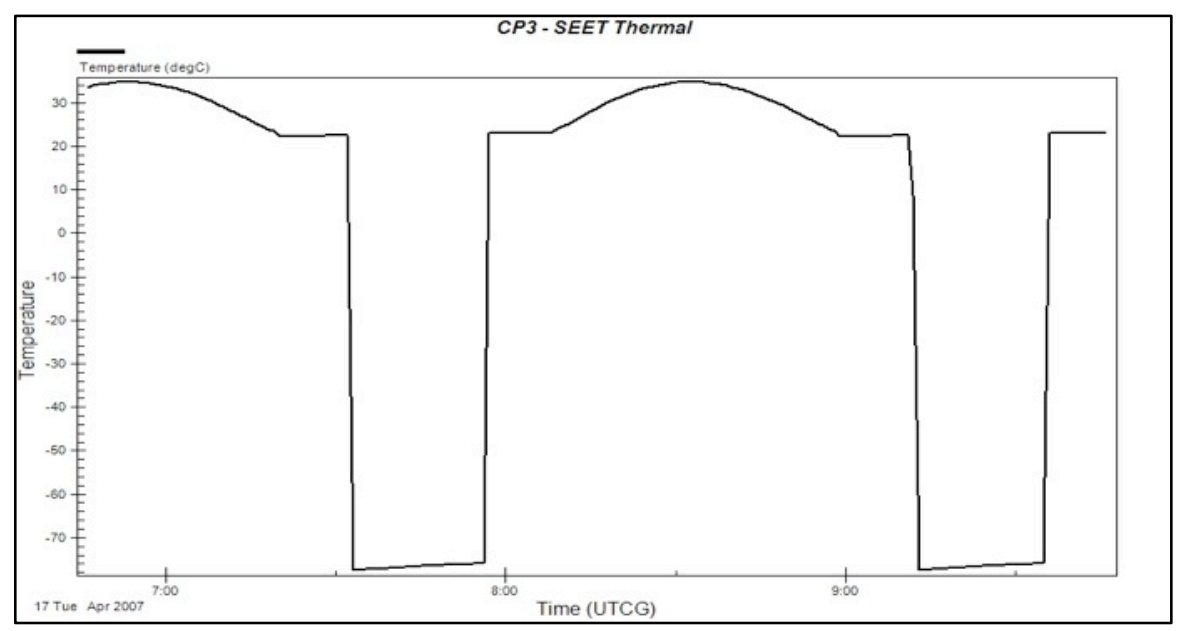

Figure 43. Baseline Three Hour Post-Launch Run (Detail View) 
As the author noted that the SEET simulations were predicting lower than expected temperatures, the decision was made for all of the simulations performed to add in the solar flux variable thus showing the flux value that SEET was using throughout the lifetime of the simulation. The figure below shows the multiple cycles that SEET predicted for the initial 12 hours of CP3's orbit. As these temperatures are realistic external exposures this clearly illustrates the demanding environment these electronics are being placed into.

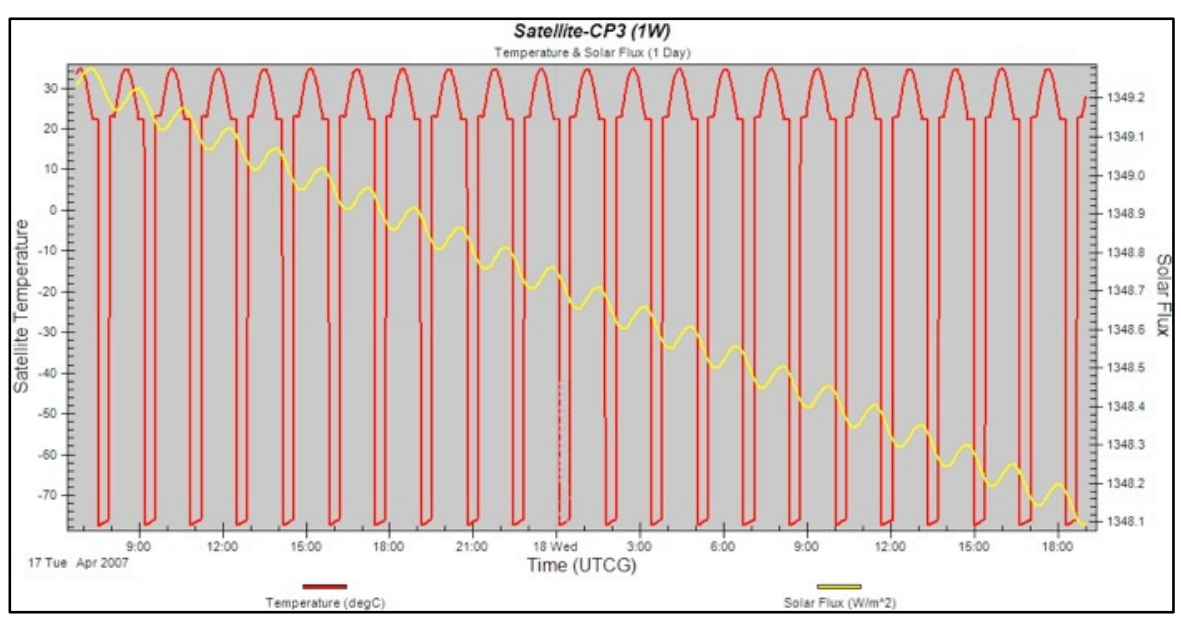

Figure 44. CP3 with 1.062W "Standard Operations" Internal Heat Dissipation

As mode $2 \mathrm{a}$ had almost no visible effect on the lower bound of the satellite temperature it was excluded from the results presented. Mode $2 \mathrm{~b}$ had a more significant effect and is shown below in figure 45 . The lower bound for the satellite temperature was decreased by over $10^{\circ} \mathrm{C}$ due to the extra heat being generated. 


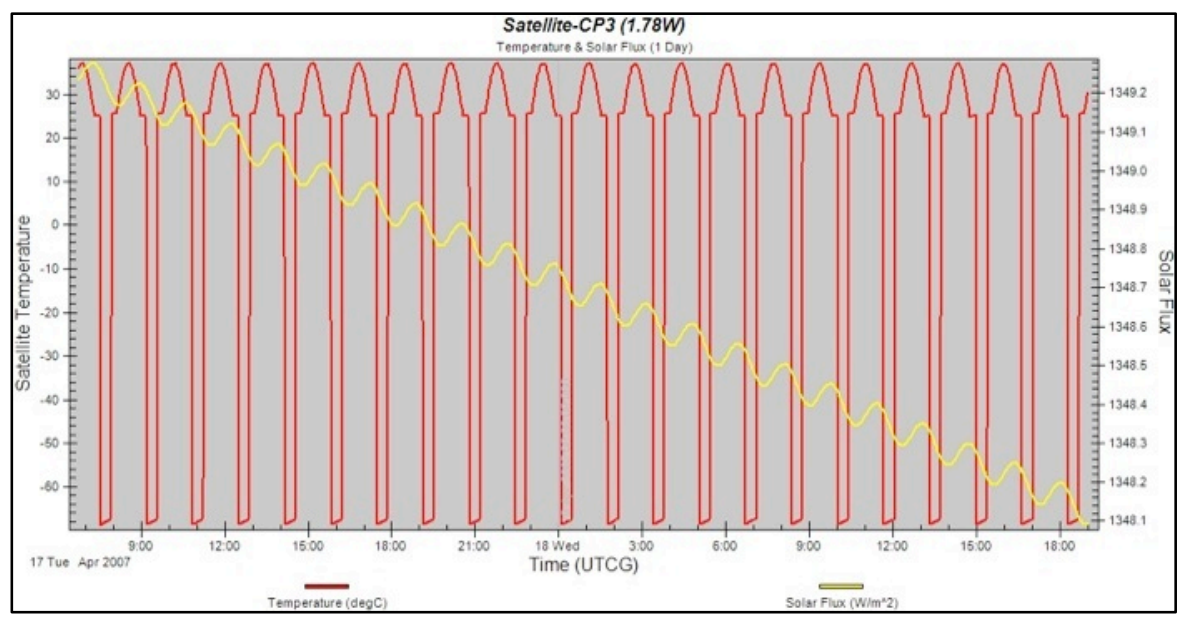

Figure 45. Mode 2b. Increased 60s Beacon Rate with 1.78W Internal Heat Dissipation

The next significant mode that showed an increase worth noting was mode 5 and the total increase for this mode was found to be over $15^{\circ} \mathrm{C}$. It was during this mode that the upper bound of the spacecraft's observed temperatures really began to spike. SEET does not have the capability to deal with step function inputs and as such the upper bounds for modes $4 \mathrm{a}, 4 \mathrm{~b}, 4 \mathrm{c}$ and 5 are not true representations of the satellites temperature (as the heat being generated by these modes would be eliminated before leaving umbra conditions).

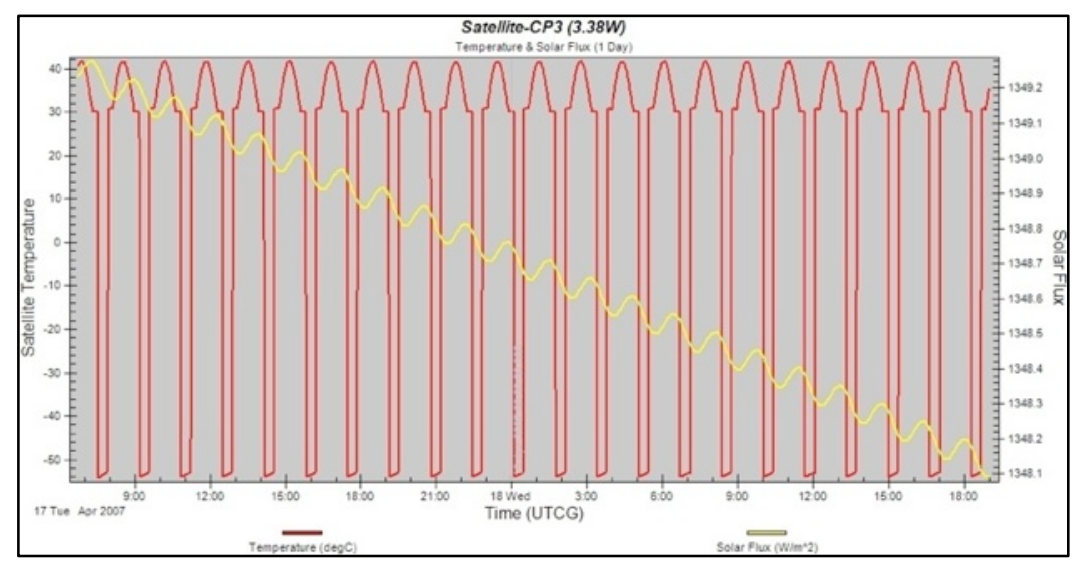

Figure 46. Mode 5 - Increased Data Drop Simulation with 3.38 W Internal Heat Dissipation 
The final cases run using SEET were for the ADC modes $4 a, 4 b$ and $4 c$. These modes showed significant improvements moving the initial low temperature prediction from $-74^{\circ} \mathrm{C}$ up to $-41^{\circ} \mathrm{C}$, a 33-degree improvement and a reduction of $31 \%$ in the temperature range of the satellite while operating under mode $4 a$.

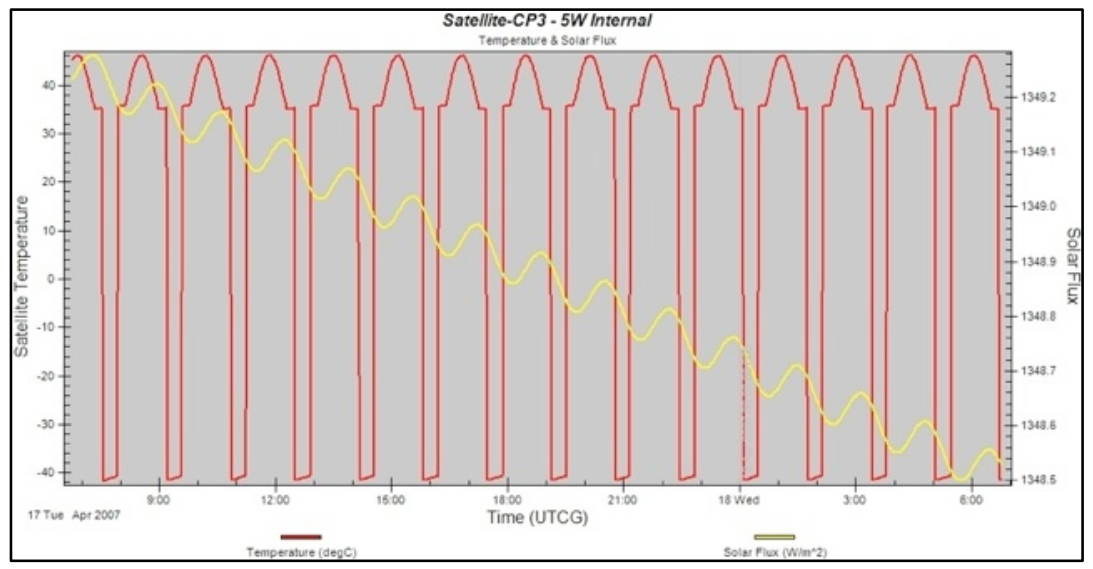

Figure 47. Mode 4a. ADC System "ON" with 5.09 W Internal Heat Dissipation

For Mode $4 \mathrm{c}$ the low temperature condition was found to be $-33^{\circ} \mathrm{C}$. In this "best case" mode the on-orbit temperature difference was reduced by $38 \%$.

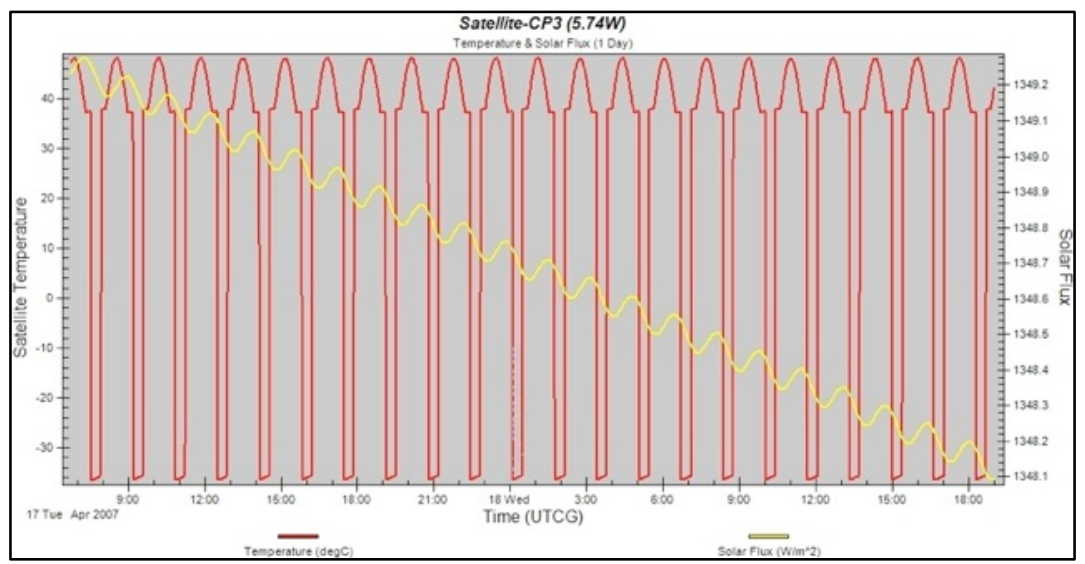

Figure 48. Mode 4c. ADC System "ON" and 60s Beacon Rate with 5.74 W Internal Heat Dissipation 
The satellite theoretically has approximately a 9 Watt heating capability, unrealistic for any operations as it causes a certain "brown-out" condition, it was simulated in SEET as a "what-if" condition as extra analysis if SEET would not harm any physical hardware (as testing this mode in T-VAC most certainly would). The $9 \mathrm{~W}$ heating condition resulted in an extremely favorable lower bound of $-14^{\circ} \mathrm{C}$ and reduced the on-orbit SEET predicted temperature swing by $56 \%$.

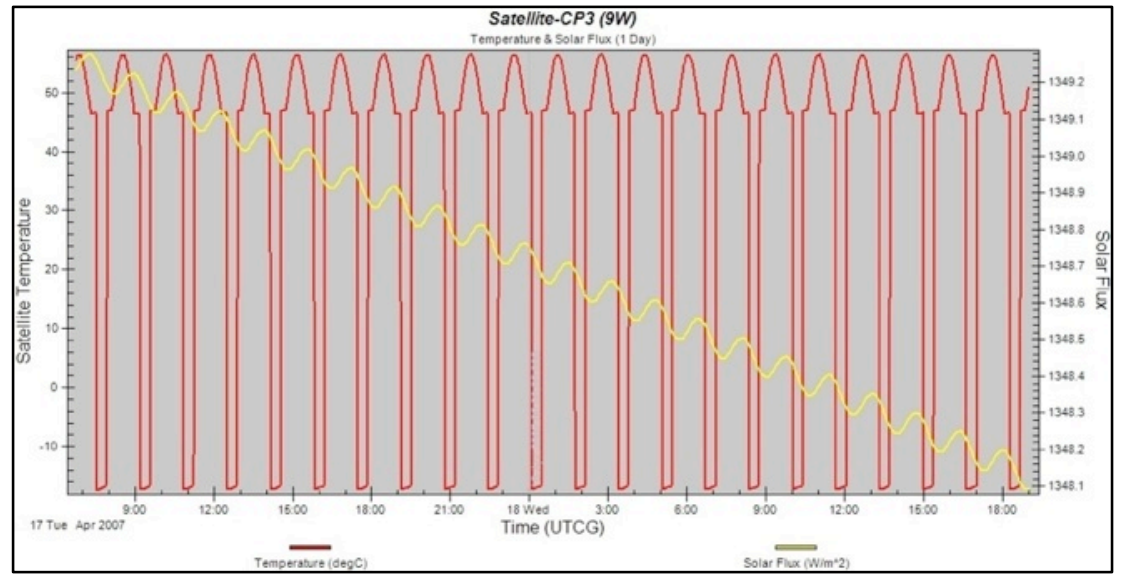

Figure 49. Heating Case with 9 Watt Internal Heat Dissipation 


\subsubsection{Heat Flux Path Mapping via Resistor Model}

Thermal energy has the unique property of being able to use electrical resistance concepts and theories. Just as voltage from high to low across a resistor cause current, temperatures from high to low across a material cause heat flux. Heat flux can thus be represented as current, temperatures as voltages, material resistances (material through which heat flux is flowing) as resistors, heat sources as current sources and finally thermal masses as capacitors.

Using these substitutions one can take the physical layout of the system in question and apply both Fourier's Law along with Equation 7 (Section 3.3.2) to create a representation of the satellite system's heat transfer paths and resistances. The two figures below, 50 and 51 were taken from a surface mount component supplier and give a detailed view of how this theory is applied. Figure 50 shows the physical component layout and the "resistances" it must pass through in order to reject heat. 


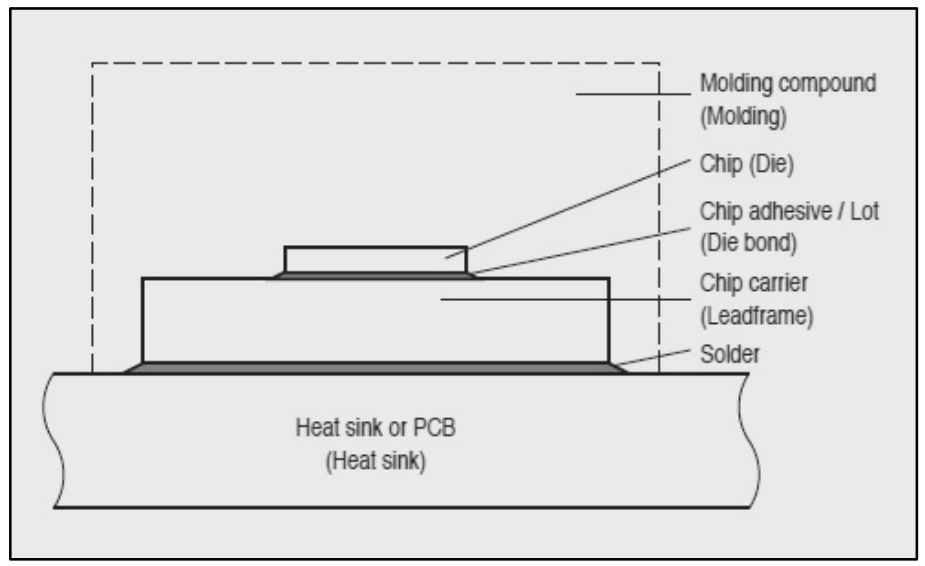

Figure 50. Surface Mount Component (PIC) Material Layers ${ }^{21}$

Figure 51 below shows the equivalent electrical circuit that equates to the heat transfer for the physical scenario above. This figure also shows the necessary electrical components to not only calculate the equivalent resistance but also to simulate the flow of heat and different temperatures in the system.

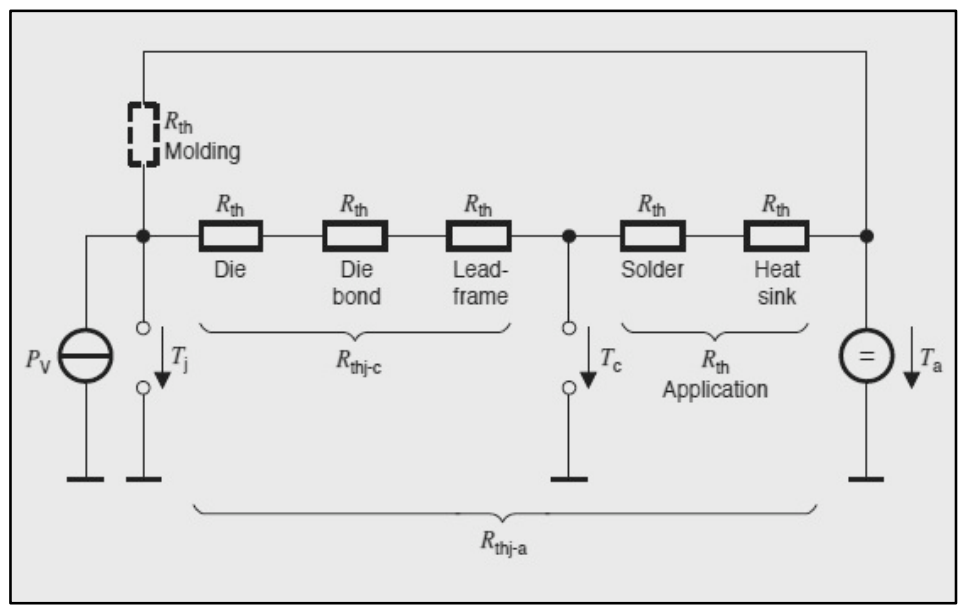

Figure 51. Surface Mount Component Resistance Network \& Heat Flow (Example) ${ }^{21}$ 
As an example, the graphic below (figure 52) was created to show the heat flowing to and from a side panel and into the CubeSat. In this figure $R_{S}$ stands for the resistance of a side panel, $\mathrm{R}_{4-40}$ stands for the resistance of each of the bolts connecting the panel to the structure, and $R_{S T}$ represents the aluminum structure of the satellite. This analysis follows the reference from Gilmore and Phillips. ${ }^{2}$ The thermal resistance for $4-40$ steel fasteners in Gilmore is $3.79^{\circ} \mathrm{C} / \mathrm{W}$.
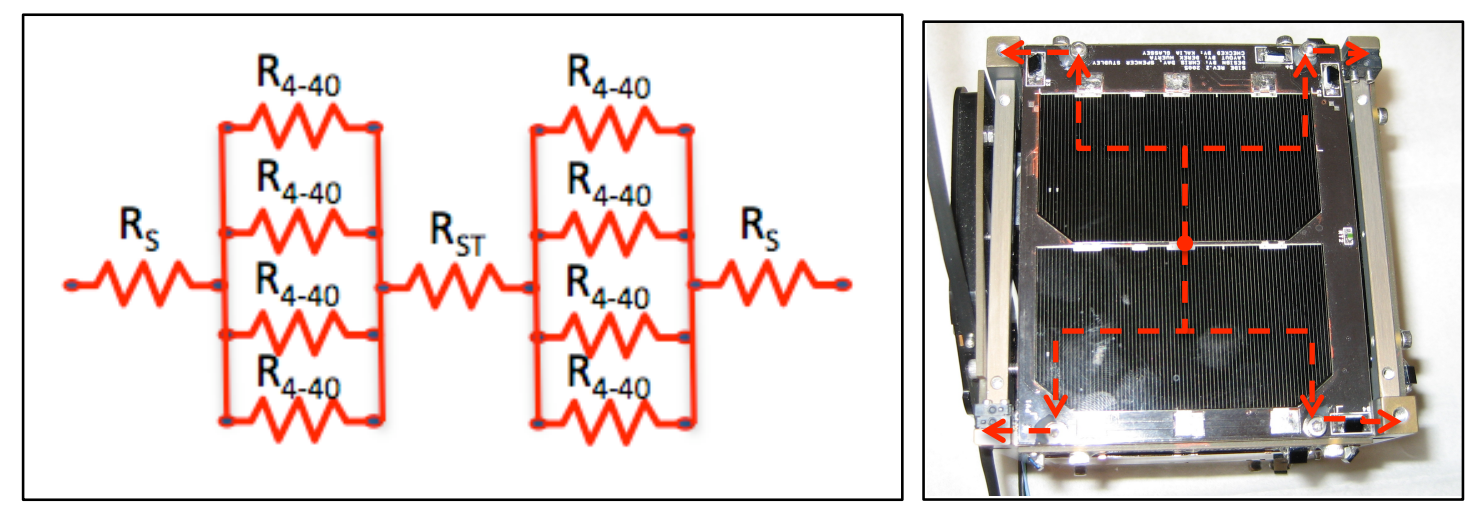

Figure 52. Resistance Model for CubeSat Side-Panel with Example Layout Using this model one can calculate the resistance to the flow of heat that is experienced from the center of an exposed panel, into the structure of the satellite and back into another side panel (center). The equivalent resistance is calculated using the equation below.

$$
R_{E Q}=R_{S}+\frac{R_{4-40}}{4}+R_{S T}+\frac{R_{4-40}}{4}+R_{S}
$$

\section{Equation 20. Equivalent Resistance from Side Panel to Side Panel (Lateral)}

This calculation, along with Fourier's Law for $R_{S}$ and $R_{S T}$, generates equation 21 where $\mathrm{x}_{\mathrm{S}}$ denotes the distance the heat must travel in the side panel, $\mathrm{k}_{\mathrm{S}}$ denotes 
the conductivity of the side panel, $A_{S}$ denotes the cross-sectional area that the heat can flow through. The structural resistance follows the same denotation scheme.

$$
R_{E Q}=\frac{x_{S}}{k_{S} * A_{S}}+\frac{R_{4-40}}{4}+\frac{x_{S T}}{k_{S T} * A_{S T}}+\frac{R_{4-40}}{4}+\frac{x_{S}}{k_{S} * A_{S}}
$$

\section{Equation 21. $\boldsymbol{R}_{E Q}$ from Side Panel to Side Panel (Lateral) with Fourier's Substitution}

Using the equation above the equivalent thermal resistance was calculated (Appendix I) to be $23.3 \mathrm{~K} / \mathrm{W}$ or $23.3^{\circ} \mathrm{C} / \mathrm{W}$. This is surprisingly consistent with on-orbit panel-to-panel results. The on-orbit dispersion between an illuminated "hot" side panel and a non-illuminated "cold" panel is in a similar range (shown in figure 53). While the heating load is dispersed and not a uniform $1 \mathrm{~W}$ condition, if we use a rough measure of solar power incident on one panel $(\sim 1 \mathrm{~W})$ this resulting resistance shows that the model is reasonable. 


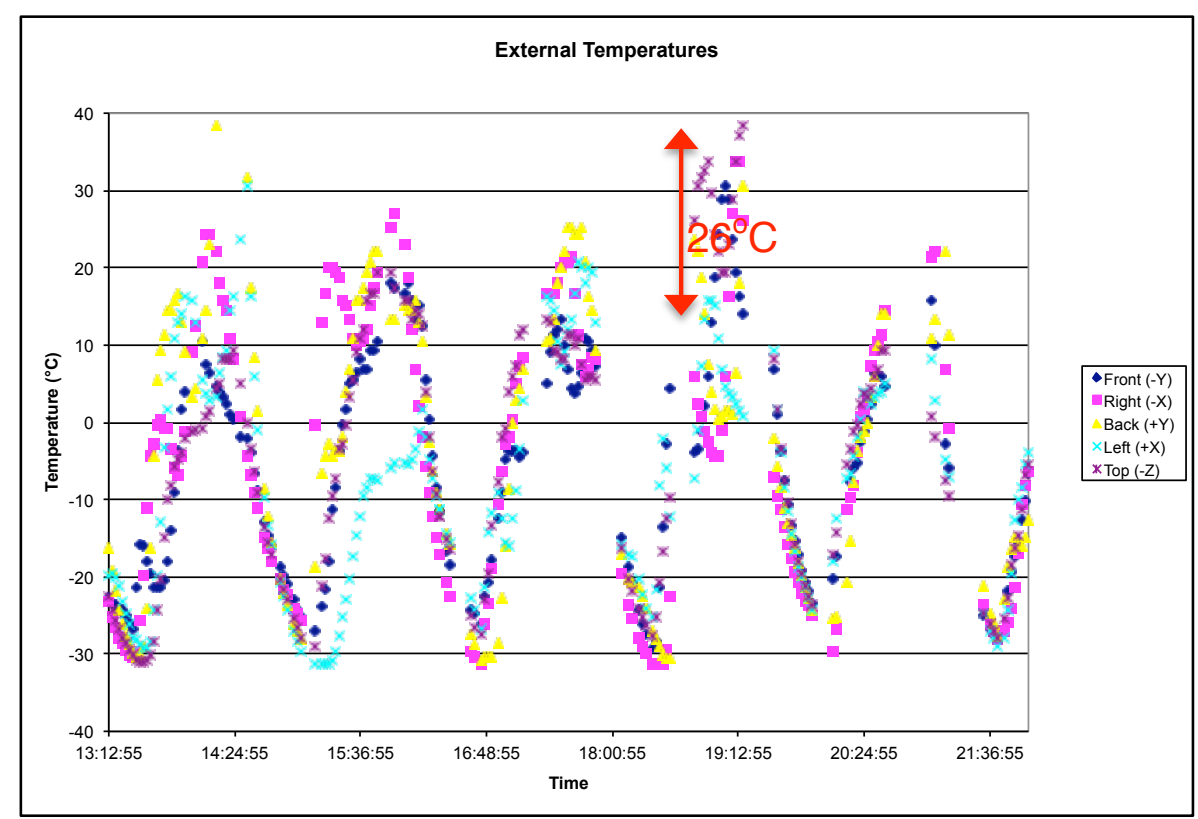

Figure 53. Resistance Model Comparison

Another measure of thermal resistance for the CP3 CubeSat is between batteries mounted on an interior panel and the exterior of the satellite. This is a far more convoluted resistance network as it involves both contact resistance and thermal radiation as a heat transfer forms. The model is shown below next to a clamshell shot of the CP3 structure showing the approximate heat transfer paths in red.
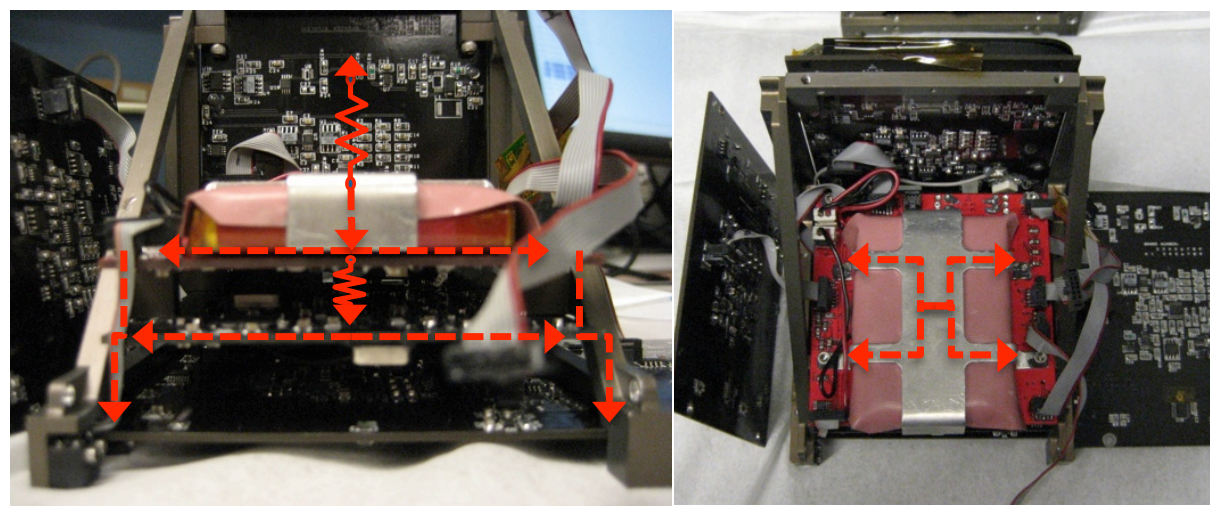

Figure 54. Battery Panel to Structure Heat Paths 
An equivalent resistance for this scenario would involve capacitors to simulate the battery thermal mass, resistors in parallel running through the aluminum holder, through contact with the PCB below and through radiation to both of the PCB boards it views (one from the aluminum holster, the other from the $-Z$ of the power PCB). Next there would have to be additional serial resistors to carry the conducted and re-radiated heat into the structure of the satellite and on to an external panel from where it could be rejected or accepted depending on the temperature difference (or in electrical modeling the voltage difference). As modeling in this manner was not seen as accurate enough to warrant inclusion in the thesis beyond the simple modeling performed as an example in figure 52 and equation 21, this battery to exterior scenario was not modeled.

For reference the view factor between interior PCBs in the CubeSat form factor also included in Appendix I.

\subsubsection{Finite Element Model Generation and Solutions}

Finite element modeling can be extremely useful. It is one of the primary methods used by modern engineers for performing analysis on objects with multiple boundary conditions, various materials, and complex geometry. It provides engineers with a valuable tool to create a visual representation of their hardware's condition. The computer aided engineering tools used for this section of the thesis are COMSOL and COSMOS. 
COMSOL is a multi-physics simulator, originally built from Matlab libraries for complex physics simulations. COMSOL evolved into a graphical interface tool resting on those libraries that allows extremely complex and highly integrated simulations to be run. As an example, if you wanted to simulate the electrical resistance of a circuit while under heating loads and inside a magnetic field, COMSOL would be the tool to use. However, it has two major downfalls. First, creating and importing computer aided design (CAD) files is extremely prone to issues. Second, the ability to create physical geometries inside of COMSOL is limited and painstakingly slow. With these issues aside, for simple geometries but complex scenarios it is a powerful simulation tool.

The next software package utilized was COSMOS. COSMOS is a Dassault Systems product that is built into the SolidWorks PE software bundle. It is a widely used computer aided engineering tool for both mechanical and thermal analysis. Previous versions (pre-2006) did not incorporate radiative heat transfer but the 2007 version (used for this thesis work) did incorporate radiative heat transfer and the detailed engineering calculations to support it. COSMOS' major advantage to other CAE packages is that it integrates very well with $C A D$ packages (both SW and others). The usual issues of joint failures, angle mismatches or IGES/STEP corruption occurring is far less prevalent when using COSMOS. Therefore, for models with fairly simple thermal or mechanical processes, COSMOS is one of the best available tools. 
The first step was to decide which portions of the satellite needed to be modeled and to what degree of accuracy they should be modeled. Thermal simulations in both COMSOL and COSMOS are very memory and processor heavy, taking on the order of hours to converge on a transient simulation of only minutes.

The first simulation run for the thesis incorporated the CP3 model shown below (excluded C\&DH board). This model was readily available from a previous project done in SW and so it was a perfect test-case for practicing. The model was placed into a $264 \mathrm{~K}$ radiative "enclosure" (slightly higher than the predicted analysis in section 3.3.3) and the appropriate emissivity and absorptivity values were placed on both the aluminum structure and the payload boards. The payload C\&DH board was given an internal heat flux of $.28 \mathrm{~W}$ applied to the PCB directly. The battery board was given the remaining $.72 \mathrm{~W}$ of heat flux. This is a gross simplification because the actual heat being generated is primarily on the C\&DH board that would typically be modeled above the battery board. The battery board only accounts for about $6 \%$ of the total heat generated internally during discharge conditions (about $.07 \mathrm{~W}$ ) but since this was to be a training run for simulations the simplification was found to be acceptable. 


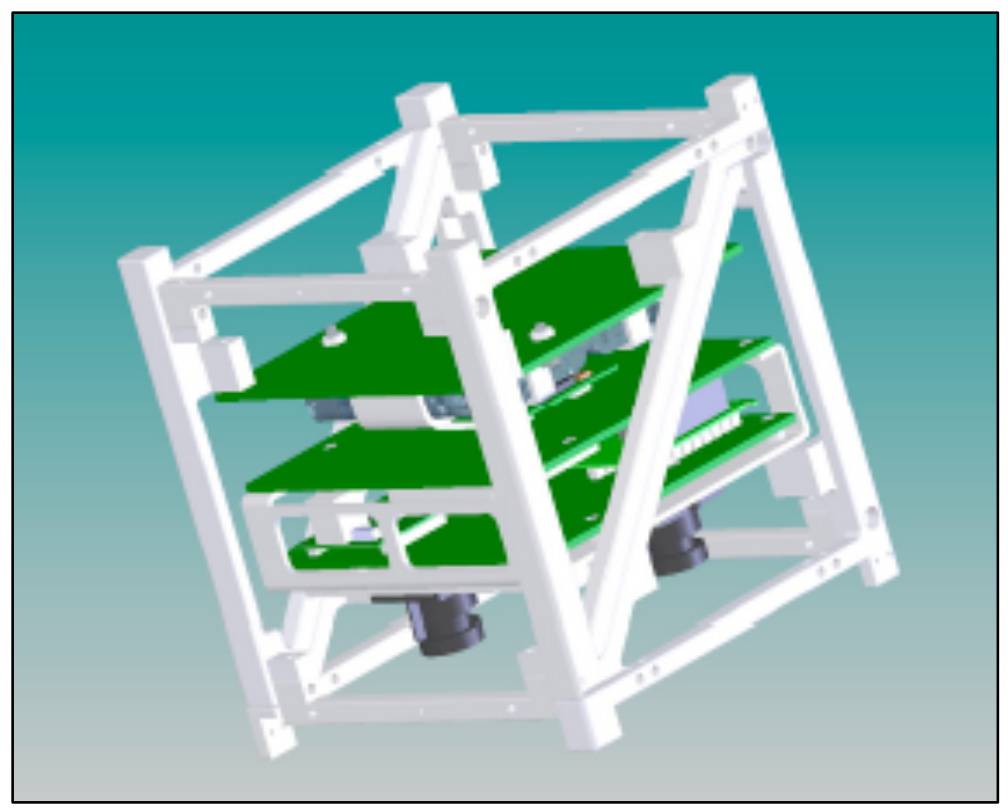

Figure 55. CP3 Internals Exposed - SolidWorks Model w/o C\&DH Board and Side-panels

The aluminum structure was giving the proper conductivity (128 Watts/mK) but no heat flux. Contacts between the panels and the structure were given a resistance of $3.80 \mathrm{C}$ per Watt, consistent with $4-40$ bolts. All boards were allowed to re-radiate to one another freely with IR emissivity and absorptivity values generated by the testing done in section 3.2.2 adjusted for the results of the study in 3.2.1 and 3.2.3. The model automatically generates independent view factors using the system geometry so the author was not required to develop those factors for the simulation.

The PCB conductivity for every FEA simulation was set to the value provided by Sierra Express for the ISOLA FR406 material with an adjustment for ground plane conductivity one ounce per foot or $1 \mathrm{P}$ on the CP payload and 
C\&DH boards and 2 ounces or 2P (4 ground planes total) on the EP\&H board (Appendix B).

\begin{tabular}{|l|c|c|}
\hline \multirow{2}{*}{$\begin{array}{c}\text { \# OF BURIED 10z } \\
\text { Cu PLANES }\end{array}$} & \multicolumn{2}{|c|}{$\begin{array}{c}\text { PCB EFFECTIVE THERMAL } \\
\text { CONDUCTIVITY, W/m-K }\end{array}$} \\
\cline { 2 - 3 } & $\mathbf{x ~ \& ~ y ~}$ & $\mathbf{z}$ \\
\hline NONE (FR-4 ONLY) & 0.87 & 0.310 \\
\hline ONE PLANE (1P) & 9.4 & 0.317 \\
\hline TWO PLANES (2P) & 18 & 0.324 \\
\hline
\end{tabular}

Figure 56. Conductivity Across and Through an FR4 multilayer $\mathrm{PCB}^{22}$

The effective thermal conductivity for standard FR4 PCB material is shown above along with optional copper sub-planes. While challenging to design against, adding just a one ounce copper ground plane to the board has a massive effect increasing the in-plane $(X-Y)$ conductivity of the board by over 10 times. As would be expected, conductivity in the $Z$ direction is only minimally affected by the addition. This one-ounce addition would correspond to a 1.4 millimeters layer of copper in the board. The figure below illustrates where these copper ground planes are typically located and how they interface with the PCB. 


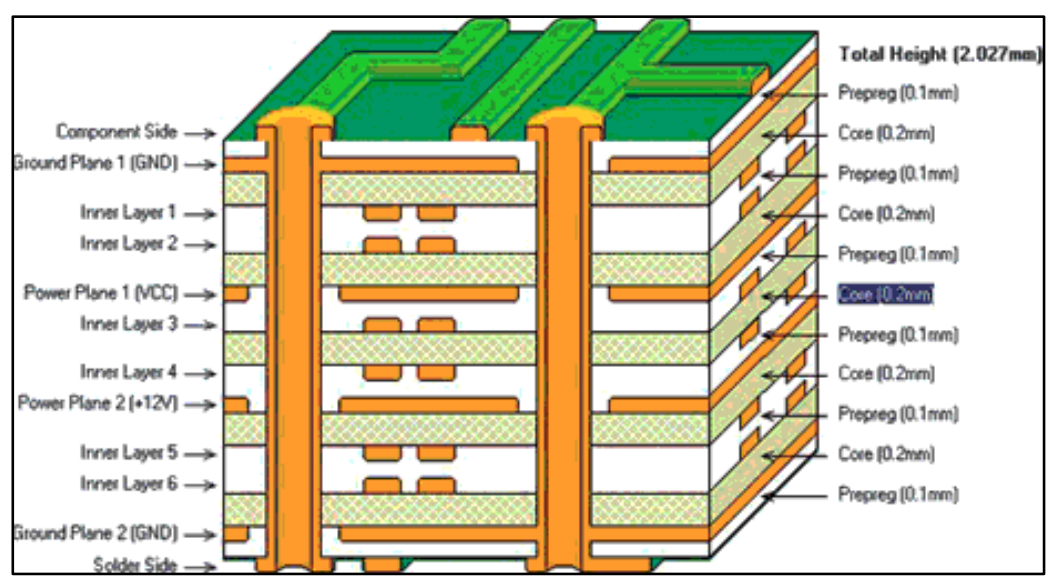

Figure 57. 6-Layer Printed Circuit Board Design \& Ground Plane Illustration ${ }^{23}$

For reference a ground plane in PCB assembly is not something primarily designed to spread heat it is a layer of copper that appears to most signals as an infinite ground potential. This helps reduce noise and helps ensure that all integrated circuits within a system compare different signals' voltages to the same reference potential. ${ }^{23}$ 


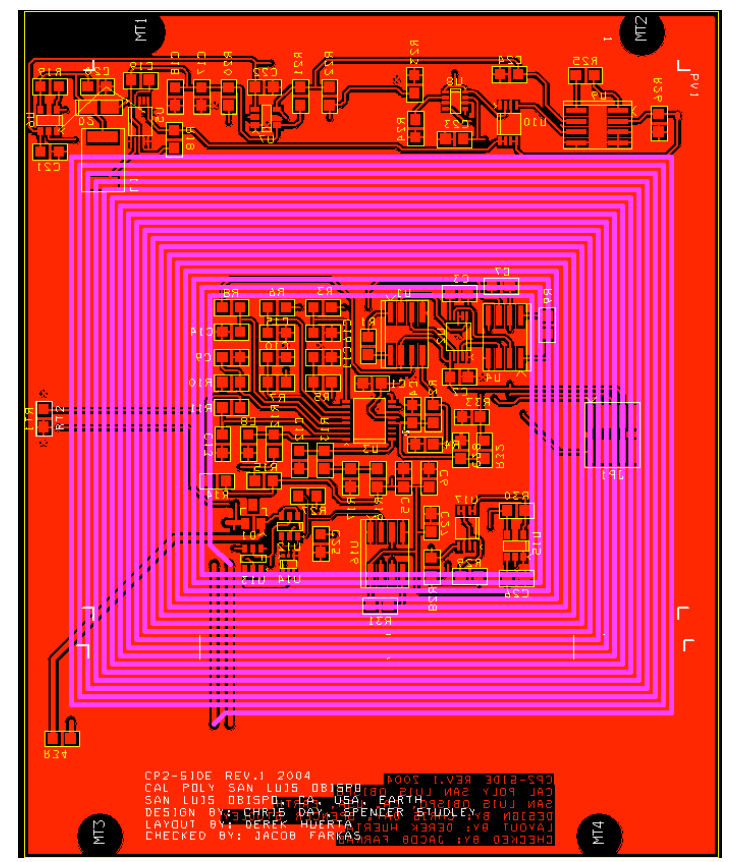

Figure 58: Magnetorquer traces in the CP side panels

No data was available to quantify the weight of the copper trace inside the solar panel magnetorquer coils (shown above in figure 58) so no adjustment was made for their thermal conductivity. From a qualitative standpoint they are definitely adding some amount of in-plane thermal conductivity and a future project to quantify the amount is suggested. Trying to model the actual ground traces inside the PCB (example shown below: Figure 59 NPS CubeSat) was decided to be outside the scope and requirement of this thesis as the 1P ground planes in the panels provided the majority of the thermal conductivity in the board and excluding the individual traces was conservative and would only serve to underpin the thesis results. 


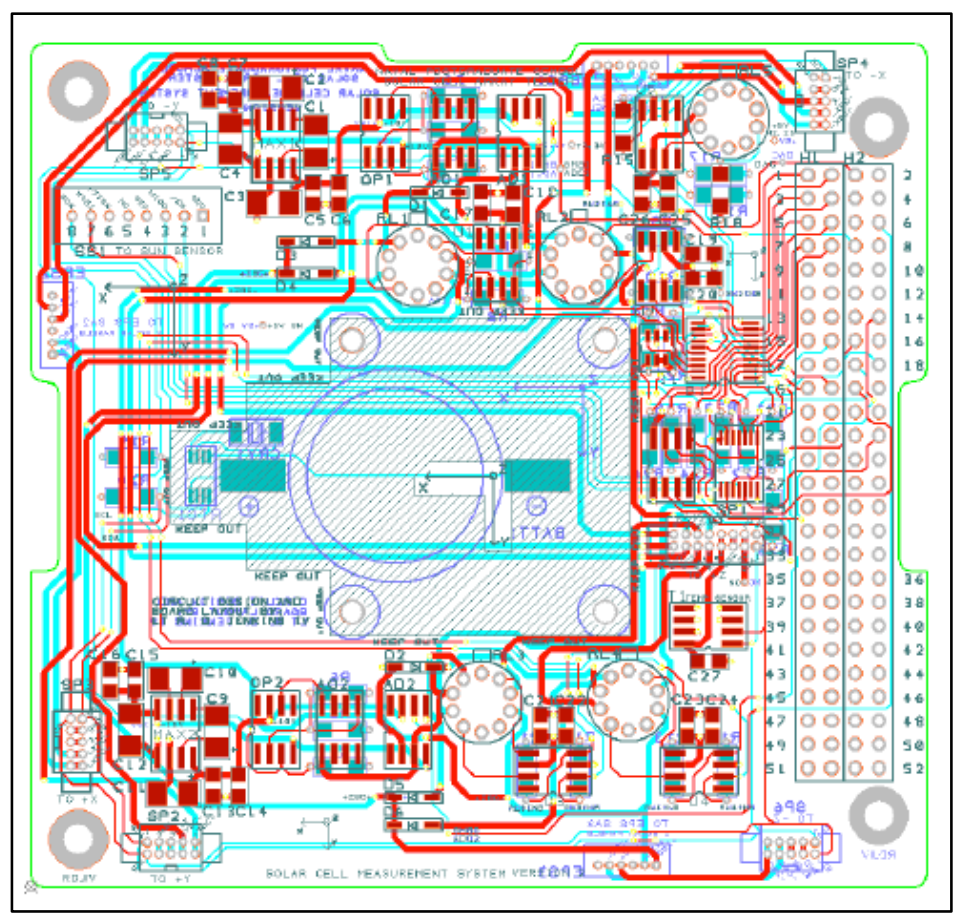

Figure 59. NPS CubeSat Payload PCB Board Showing Layers and Traces ${ }^{9}$

After implementing the correct PCB conductivity for the payload and C\&DH boards the model was set to run with a 33-minute "cold" case to simulate the eclipse period of the CP3 LEO orbit. The model ran for approximately three hours before COSMOS crashed. This gave the author his first lesson in thermal FEA modeling, simplify, simplify, simplify. After attempting to re-run the simulation twice with no success the author decided that further FEA analyses would use the simplest geometry possible that would still deliver accurate results.

The most important data points that the FEA was required to provide back were confirmation of a reduction in the thermal gradients across boards by mode (verification criteria 1.2). With the analysis performed in section 3.3.1 this could 
be done accurately at the board level without requiring integrated and overly complex model geometry.

The first board to be analyzed and arguably the most critical was the $\mathrm{CP}$ C\&DH board shown in the figure below. First a simplified version of the board was modeled based on the following assumptions and layouts and then all the necessary conditions and properties were placed into COSMOS for the simulation.

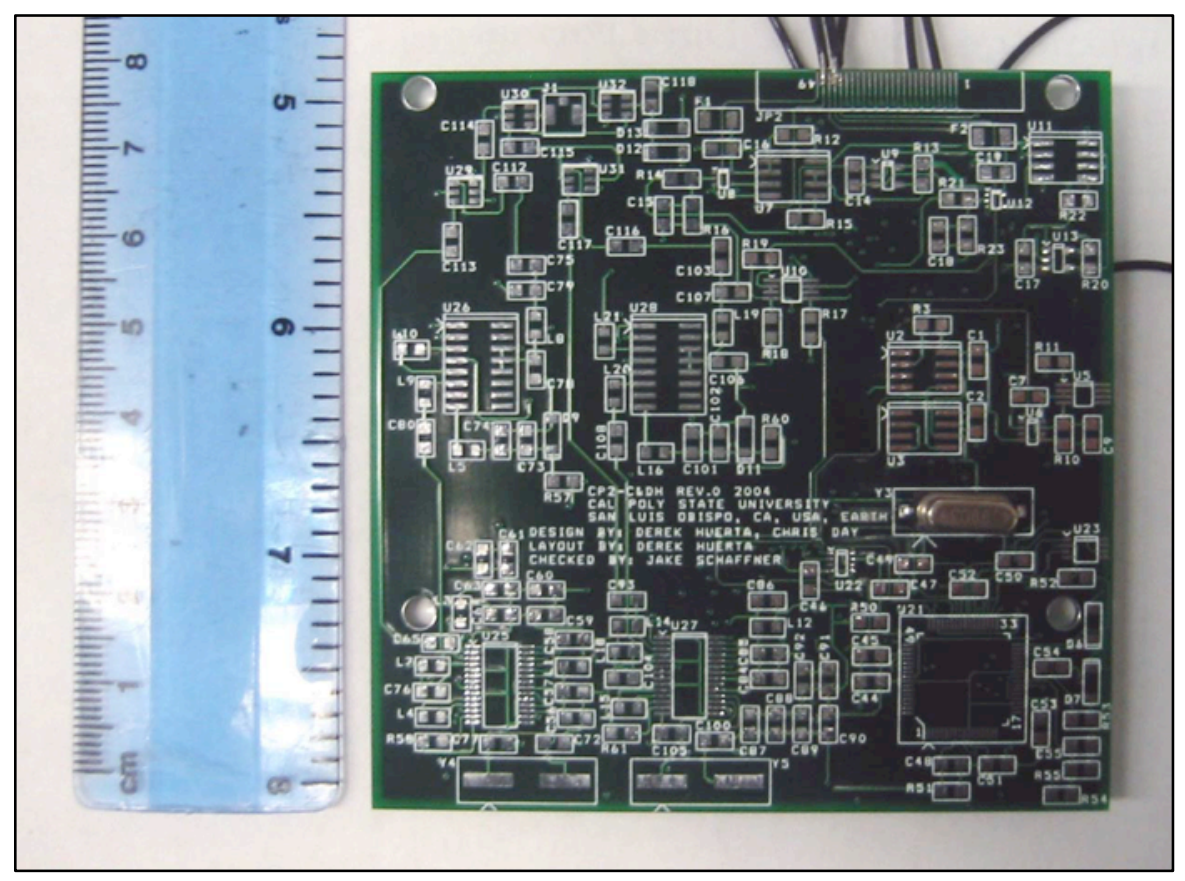

Figure 60. Layout for CP2 C\&DH Subsystem PCB

Figure 60, above, shows the command and data handling (CDH) board for the CP satellite, which is where the communications subsystem is physically located. In the lower left corner are the footprints for the redundant RF 2117 amplifiers and in the middle left are the footprints for the redundant $\mathrm{CC} 1000$ devices. The 
lower right corner shows the footprint of a single, PIC18LF6720. The second (redundant) processor is located on the opposite side of the board. ${ }^{20}$

For the CP3 Comm Board a COSMOS finite element analysis was performed to analyze the board heat distribution, average board temperature and component temperature differences. The first analysis uses an external radiative cold case of $-262 \mathrm{~K}$ to accurately simulate the interior view of the spacecraft boards but neglects board-to-board re-radiation heating effects.

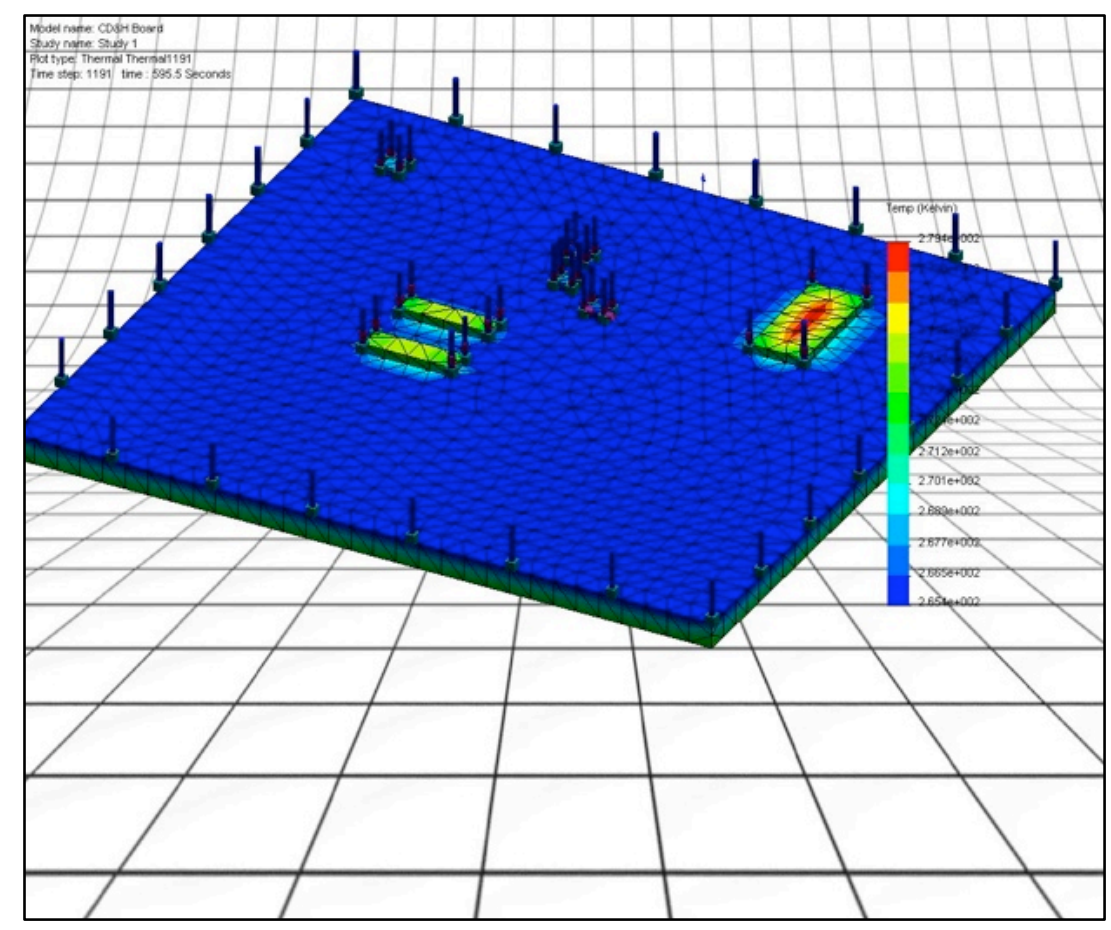

Figure 61. Cold Soak Temperatures for Normal Ops

During the normal ops simulation the board ran very cold at $265 \mathrm{~K}$ but was infamily with predicted temperatures. The RF amps and PIC 18 had $92 \%$ of the heat load applied to them (62-30 respectively) with the remainder of the internal heat load placed on four components simulating AD627 modules. The RF amps 
and PIC ran at approximately $274 \mathrm{~K}$ with the center of the PIC getting up to $279 \mathrm{~K}$. The reason for the higher center temperature is that the material used to model the PIC has a much lower conductivity than both the board and the RF amps. Also the PIC was given twice the power that the normal PIC would use because the redundant PIC on the opposite side of the board were not modeled.

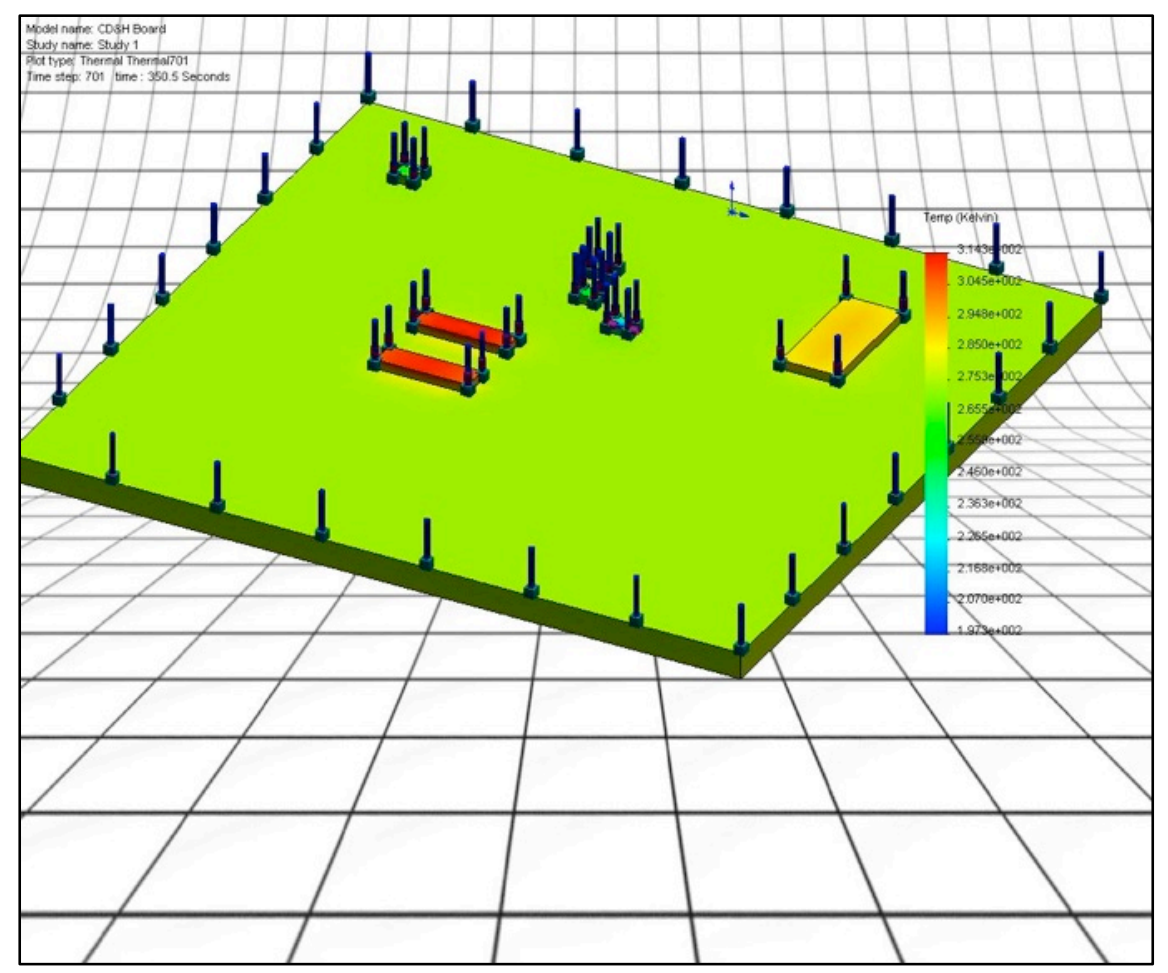

Figure 62. Cold Soak Temperatures Operating in Mode 2a

During the simulation for cold-soak with .ode 2a there was significant improvement. The board itself was running at $275 \mathrm{~K}$, an increase of $7 \mathrm{~K}$ over the non-adjusted scheme. The components also ran at more reasonable operating temperatures with the RF amps reaching $312 \mathrm{~K}$ and the PIC 18 reaching about 286K. While the RF amps did get quite high, they were still well within their normal operating temperatures. In all this reduced the total thermal cycle 
difference on the board between hot and cold cases from just over 28K down to about $18 \mathrm{~K}$. For the components, this decreased the thermal cycle even further from $38 \mathrm{~K}$ to about $23 \mathrm{~K}$. While computer aided engineering programs make simplifications in geometry, interfaces and loads, these results were still very promising and gave further evidence that changes in the operating mode of components of CP satellites could lower their thermal gradients and orbital cycling.

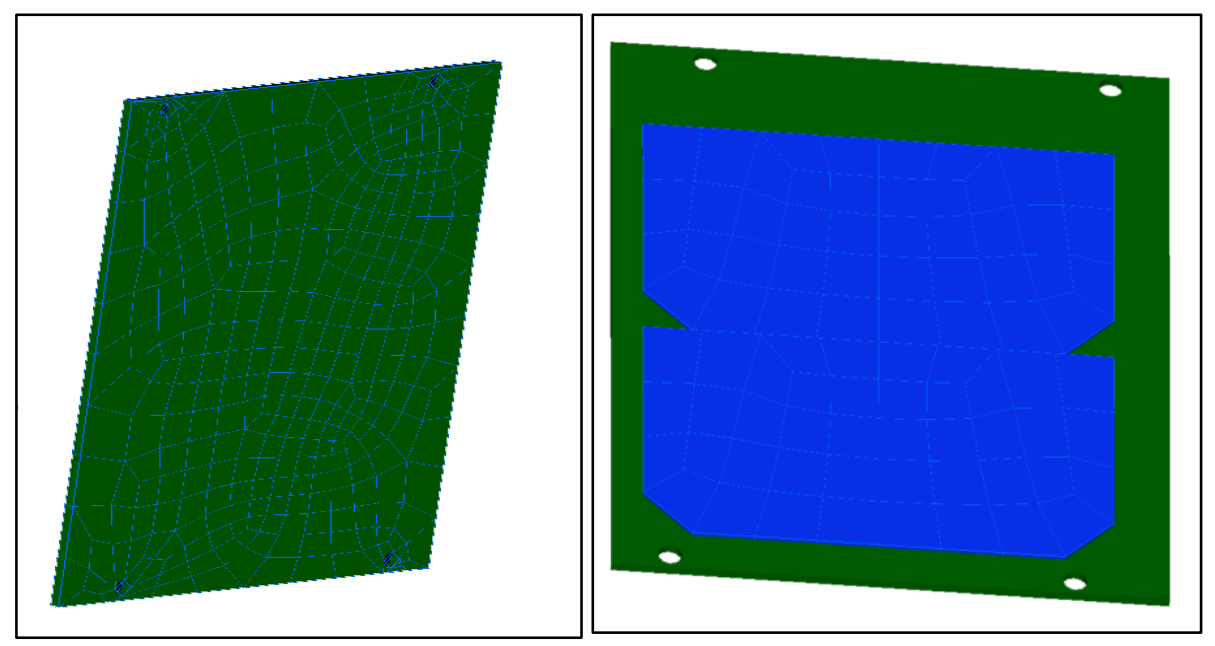

Figure 63. Solar Panel Mesh Pre-Analysis 


\subsubsection{Finite Difference Models TRASYS/TSS-SINDA}

Another method of CAE is through finite difference (FD) models. The majority of spacecraft thermal designers use one of two FD packages - TRASYS or TSS-SINDA. Both packages have extensive history in the aerospace engineering field. SINDA, which originally was named CINDA for Chrysler Improved Numerical Differencing Analyzer, originated in the 1960's and has undergone evolutions and improvements for the past forty years. There are multiple versions but the one used for the analysis in this section was SINDA85. TRASYS, which stands for Thermal Radiation Analysis SYStem, takes a userdefined model geometry, surface optical properties, orbital parameters and boundary conditions and then outputs a format compliant with SINDA input. TSS stands for Thermal Synthesizer System and relative to TRASYS and SINDA is a newer tool. It generates similar output to TRASYS but can also use ray-tracing techniques to allow for systems with reflective surfaces to be more accurately modeled. Since TRASYS was not available at the time analysis was performed the only FD model generated was done using TSS and analyzed via SINDA.

The figures in Appendix $\mathrm{J}$ show the orbital parameter insertion, material property selections and heating values. The dat file results for each mode were then individually processed in the SINDA processor. The SINDA output was in the form of a single-node temperature representing the satellites average temperature stepped across the orbit. This was done with over $>100$ orbits to ensure no significant increase or decrease in the orbital heating would occur post 
"launch" of the DNEPR 4/5 mission. Several assumptions were made in the model; the most significant giving the satellite an average $C_{P}$ that represented a mixture of aluminum and FR4 (30-70). The satellite was modeled as a sphere with one node and a mass of 1 kilogram. The initial temperature of the node was set to 70 degrees Fahrenheit. A sample of the orbital set, post settling was then selected and plotted. This was done primarily to provide another method of confirming the thesis that the temperature of the satellite could be increased onorbit with only the small amount of energy input available to its electrical power system. A representative single orbit selection for the output temperature results in each mode tested is shown in Appendix $\mathrm{J}$ with the summary of all the modes in table 18 below.

Table 18. SINDA/TSS Modes 1, 2a, 2b. 3, 4a, 4b, 4c, 5

\begin{tabular}{|r|r|r|r|r|r|r|r|r|}
\hline & \multicolumn{1}{|c|}{ Mode 1 } & Mode2a & Mode 2b & Mode 3 & Mode 4a & Mode 4b & Mode 4c & Mode 5 \\
\hline $\operatorname{Min}(\mathrm{F})$ & 1.2 & 3.2 & 5.7 & 6.3 & 24.1 & 26.2 & 27.8 & 15.6 \\
\hline $\operatorname{Min}$ (C) & -17.1 & -16.0 & -14.6 & -14.3 & -4.4 & -3.2 & -2.3 & -9.1 \\
\hline
\end{tabular}

The SINDA results were in-line with the analytical results albeit with slightly lower overall system temperature predictions. These predictions were found to be a more accurate representation of the true on-orbit system. The reason for the accuracy of the SINDA results versus the analytical results is that SINDA accounts for precise albedo loading, Earthshine and shadowing (penumbra) parameters while the analytical calculations simply use worst-case conditions. 


\subsection{Active Thermal Vacuum Testing}

Thermal vacuum testing began with the creation of standard test plan, using the TVAC SOP as a starting point. Once the testing documentation was written and a representative test profile was generated (discussed below) the first active step in thermal vacuum testing was de-integrating the satellite. This process took advantage of the clamshell structure design on CP3 to place individual thermistors inside the satellite (figure 64). These thermistors allowed for both the person at the T-VAC and the person sending commands in the lab on Marconi or Hertz to see the systems active temperature. It provided a safeguard so that during extreme testing conditions the temperatures inside the satellite could be monitored locally so that a damaging condition would not occur. 


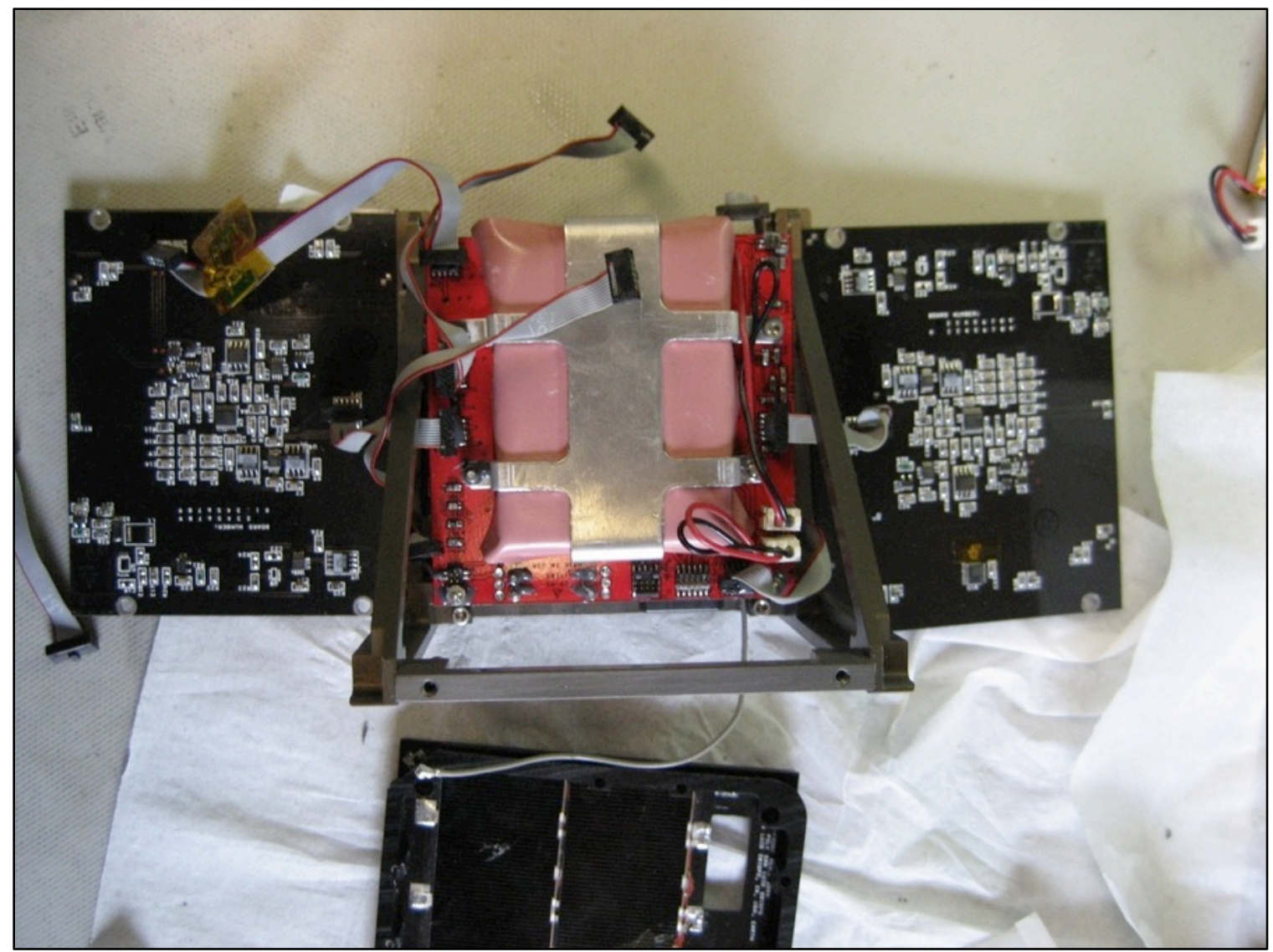

Figure 64. De-Integrated Satellite Prior to Thermistor Installation

Then using kapton approved low-outgassing tape, individual thermistors were placed into CP3 in the critical locations including the battery (1), C\&DH board (2), payload face (1) and another on the aluminum side-rail (1). These thermistors were bundled and can be seen in figure 65 below protruding from the test article inside its carrying case. 


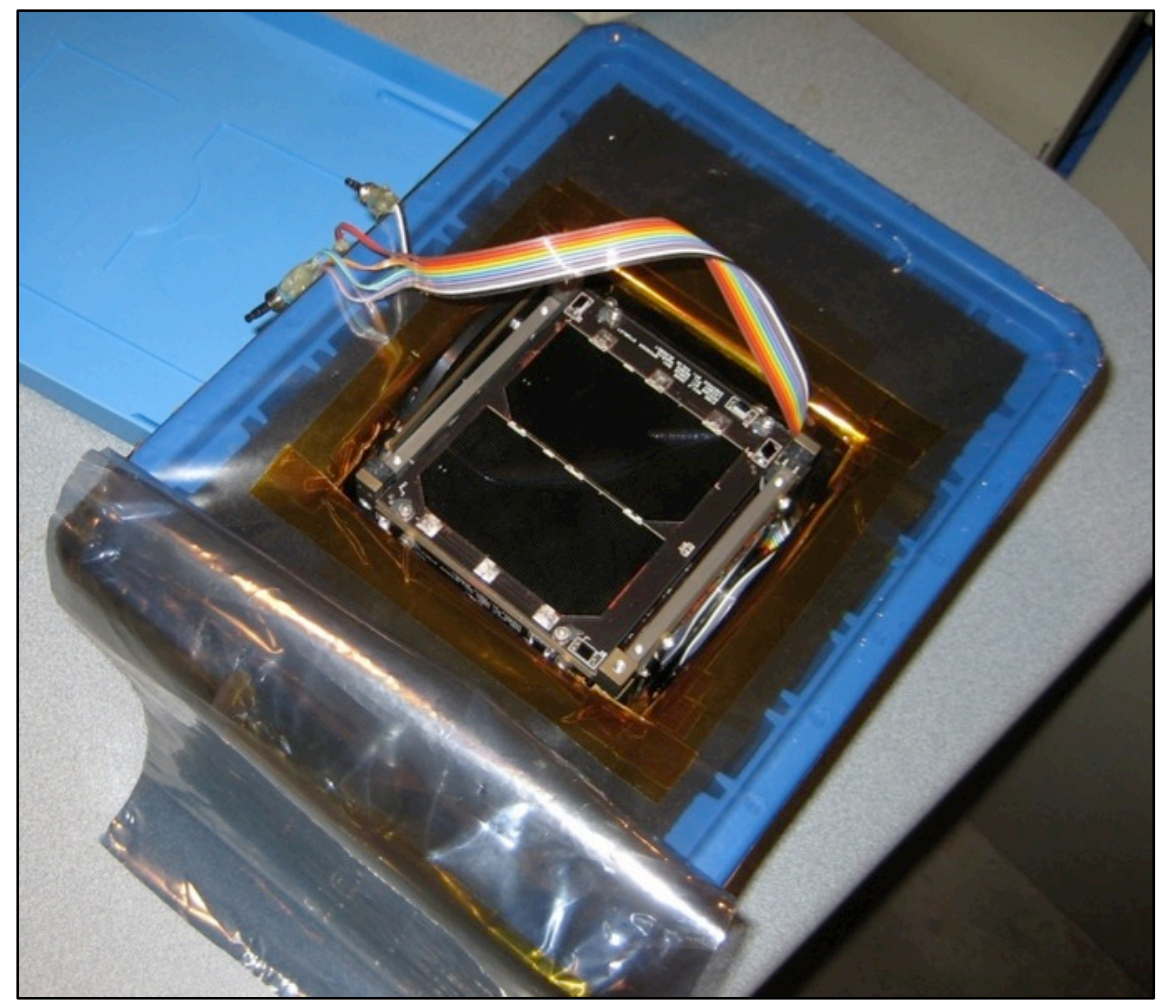

Figure 65. Thermistor Bundle Integrated Prior to Testing

To perform thermal vacuum testing the T-VAC SOP was used for each start-up procedure. Testing is performed in building 58 (Welding Building) on campus but commanding uses the ground station in Building 007 (Advanced Technology Labs - ATL). One person had to commute back to the ground station every time a command was required for testing. Luckily, other personnel were often already in the lab doing other work and would be used to send commands. The radio to the left of the Labview T-VAC PC (figure 66) was used to communicate between the lab sending the commands and the T-VAC area so the command timing could be noted in the test procedures. 


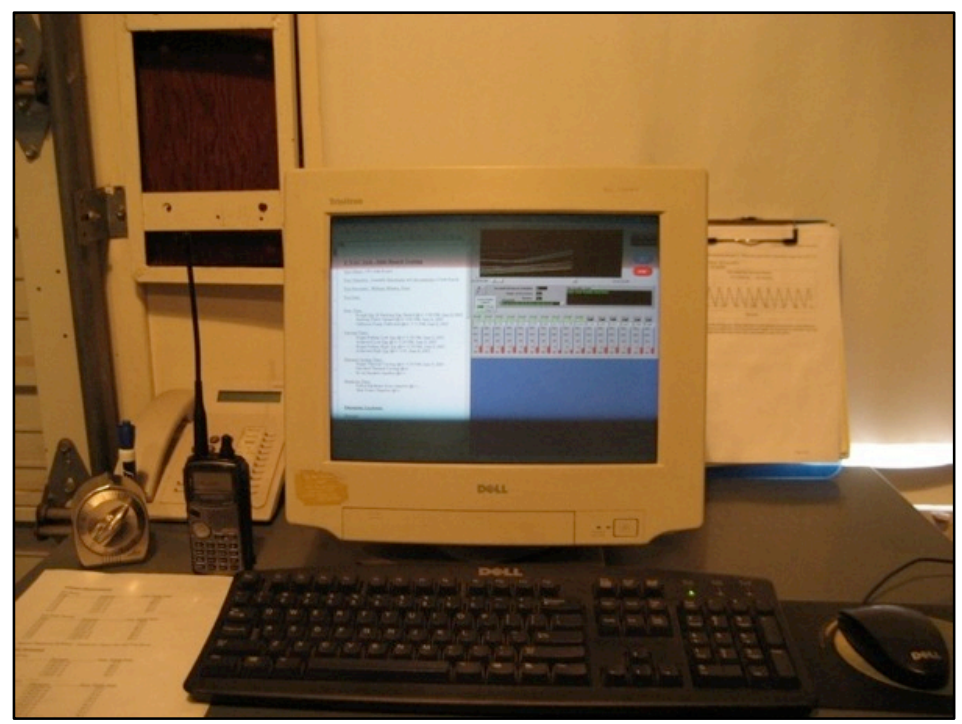

Figure 66. T-VAC PC with Labview, Test Plan \& Radio

Prior to testing the ends of the antennae were covered in kapton tape to ensure they did not ground themselves when placed inside the conductive copper shroud. While hard to see in figure 66 , the end of the tape-measure antennae has been carefully enclosed in kapton tape.

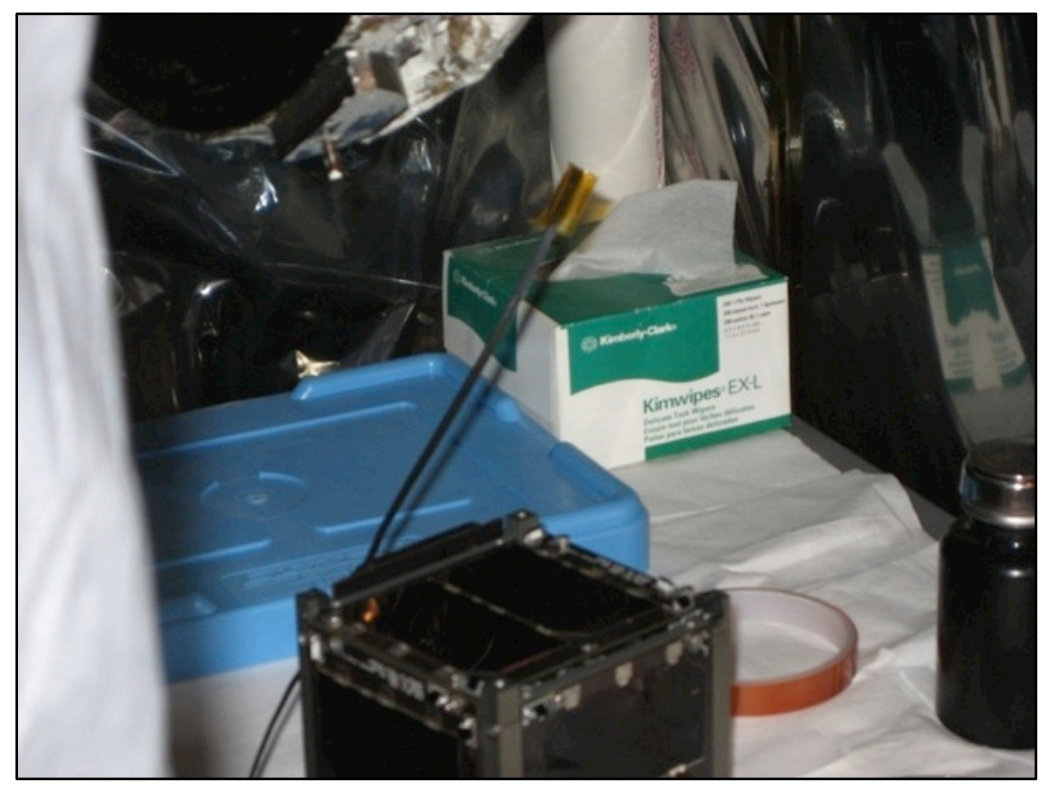

Figure 67. Antennae Taping Prior to T-VAC Testing 
Once the satellite had thermistors integrated and had been completely reassembled, personnel for the test were coordinated to be present at both the lab and the T-VAC for the duration of the testing. The satellite was removed from its carrying case once inside the antechamber at the Thermal Vacuum facility. Once removed it was checked to ensure none of the visible thermistors had settled or been moved. The antennae, which had been taped down prior to being placed into the carrying case, were released (ends still taped). Finally, before placing the satellite into the chamber its Remove Before Flight (RBF) pin was removed and then the satellite was placed into the chamber onto Teflon stand-offs.

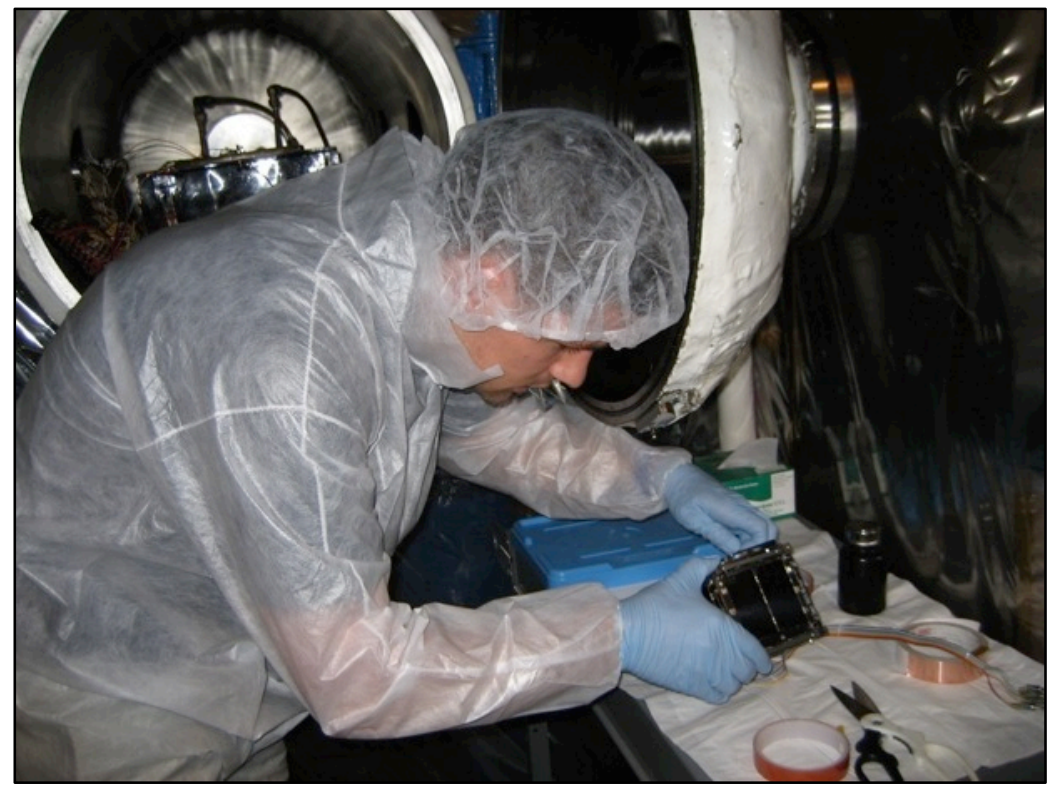

Figure 68. Preparing the Satellite for Testing; Thermistor Bundle \& Antennae Release After placing the satellite inside the chamber, the normal T-VAC standard operating procedure (SOP) was performed, checking the ring-seal on the T-VAC door, closing the door and checking that the vent valve was closed and finally 
exiting the ante-room. Once inside the secure chamber the now beaconing satellite was checked for connectivity with the ground-station. One of the test personnel exited the T-VAC area and went to the ground station (shown in figure 69), sent a test command and confirmed that they received and acknowledged or ACK back from the satellite. This was important to do before the diffusion pump was engaged and liquid nitrogen was being used. By checking prior to closeout it allowed for adjustments to be made if the link would not close or if there was a grounding issue on the satellites antennae. In this manner it could be fixed quickly and without a long penalty "cool-down" time on the vacuum chamber.



Figure 69. Marconi and Hertz, PolySat Groundstation Computers, Antennae Controllers and Radios

Once the RF link was verified to be working satisfactorily the chamber was powered on, he roughing pump was engaged, and the process of lowering the pressure inside the chamber began. Once the roughing pump was at an acceptable level, typically around 70mTorr, the diffusion pump was engaged and allowed to warm up. Typically this takes around 50 minutes to an hour to complete. Once warmed up with low enough (50-100mTorr) pressure on the 
backing pump lines, the roughing valve was opened, allowing the chamber to begin pumping down. Around the same time the liquid nitrogen dewar was opened and the lines feeding the cold trap were allowed to fill by engaging the solenoid. Next the roughing valve would be closed allowing the backing pump section to pump down and prepare. Once the cold trap was noticeable iced up the head gate was opened and the chamber began to pull high vacuum. Once the pressure could no longer be read on the standard pressure gauge the ion gauge was powered up for the remainder of the test. As soon as the ion gauge read $10^{-4}$ Torr the test could begin. Since the majority of the tests being performed for this thesis were "cold-soaks" the chamber often reached low $10^{-5}$ Torr due to the cold temperature causing molecules to lose energy and fall to the bottom of the chamber where they would be pulled out.

Once testing began, the following model was used to simulate the on-orbit environment. The chamber typically runs around $20^{\circ} \mathrm{C}$ prior to testing during the hour and a half pre-test. As high vacuum is achieved $(T=0)$ the Omega temperature controller was set to progressively lower temperatures over a fifteen minute period with the final cold temperature setting being $-60^{\circ} \mathrm{C}$. This is close to (but not outside) the safe limits of the chamber operations and progression steps were monitored closely so that the system would not have damaging thermal strain effects. This is shown in figure 70 below, Cold Bias Testing Profile. 


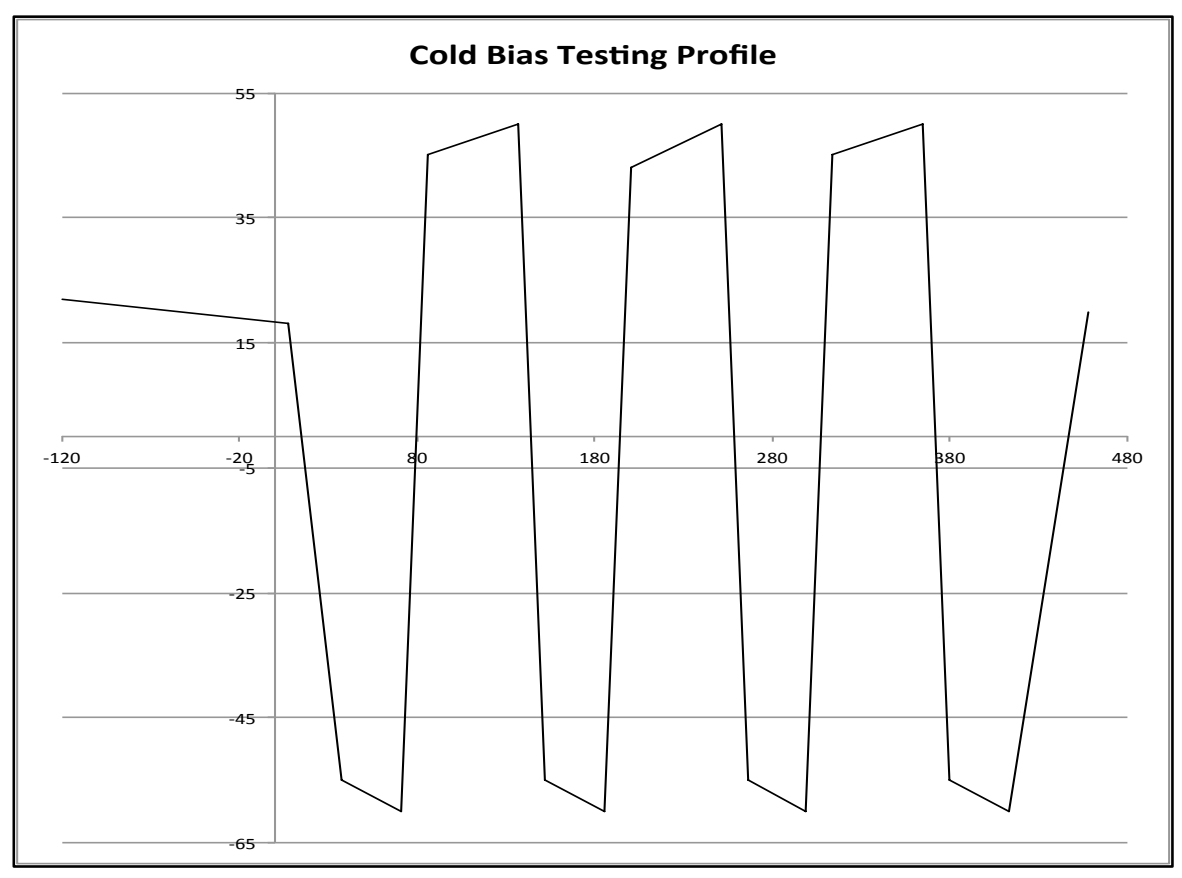

Figure 70. Cold-Bias Testing Profile

This testing profile actually uses a longer setting at the "cold" station (4566) than the satellite would normally experience on orbit (33-66). The primary reason for biasing the test parameters in this way is for conservatism. The thermal vacuum chamber testing performed is the most accurate simulation of the thesis available on the ground, significantly outweighing both analysis and CAE programs in response accuracy. However the actual transient radiative environment of space is extremely hard to mimic in a confined chamber. Unlike on orbit, inside the thermal vacuum the transition from hot to cold and vice-versa is not immediate, limiting the ability to "shock" the system. This reality limits the ability of a 33-66 setting to accurately get the satellite as cold or hot as it would on orbit. The testing keys espoused by NASA GEVS guidelines are to bias the conditions towards the scenario that is in question. Thus to add conservatism 
towards the scenario in question and more accurately mimic the on-orbit conditions every test was biased cold. The chamber itself is biased warm as it has a large thermal mass exposed to an indoor/outdoor environment anywhere from $21-24^{\circ} \mathrm{C}$. While the chamber's mass is insulated as best as possible from conduction and radiation to the outside environment it still provides a noticeable damping effect on the testing performed.

Another item that must be taken into consideration prior to testing is overshoot. Thermistors cannot be placed immediately on the heaters in question for EMI reasons as the large heater currents cause excessive noise and also due to material reasons as the heaters themselves get hot enough to melt the thermistors cabling. In the same way, the thermistor cannot be placed directly upon the liquid nitrogen piping because it would read a deceptively low temperature for either heating or cooling conditions. The Omega controller used to enable or disable heaters and the liquid nitrogen solenoid valve is reliant upon these thermistors that do not have an immediate reaction to the heating or cooling being performed. They have a 'lag' effect that can often catch new (and veteran) thermal vacuum test personnel off-guard as they ramp high or low and suddenly see that despite uncoupling the controller, the measured temperature in question continues to rise or fall. To ensure safety of the hardware and the test personnel the following inertia effects curve in figure 71 was generated based on the set points of the cold-bias testing profile in figure 71. Based on the expected lag and the known cutoff times the hardware should be safe throughout the test, 
going only slightly below $-60^{\circ} \mathrm{C}$ and slightly above $+55^{\circ} \mathrm{C}$, and the actual environment it sees is more accurately represented by the curves in the inertia effects figure. There was expectation, based on experience, that the hot portion of the testing would not actually reach the set limits in the fifteen minutes of ramp up time (safely). Since this was expected the ramp was lowered to a shallow gradient during the initial 45 minutes of hot "soak" slowly ramping from $40^{\circ} \mathrm{C}$ to $55^{\circ} \mathrm{C}$. This still met the intent of cold-biasing the test as the only outcome of this ramp being an un-realistic transient condition on orbit would be lower thermal influx during the "hot" on-orbit transient condition and an overall lower system temperature as a consequence.

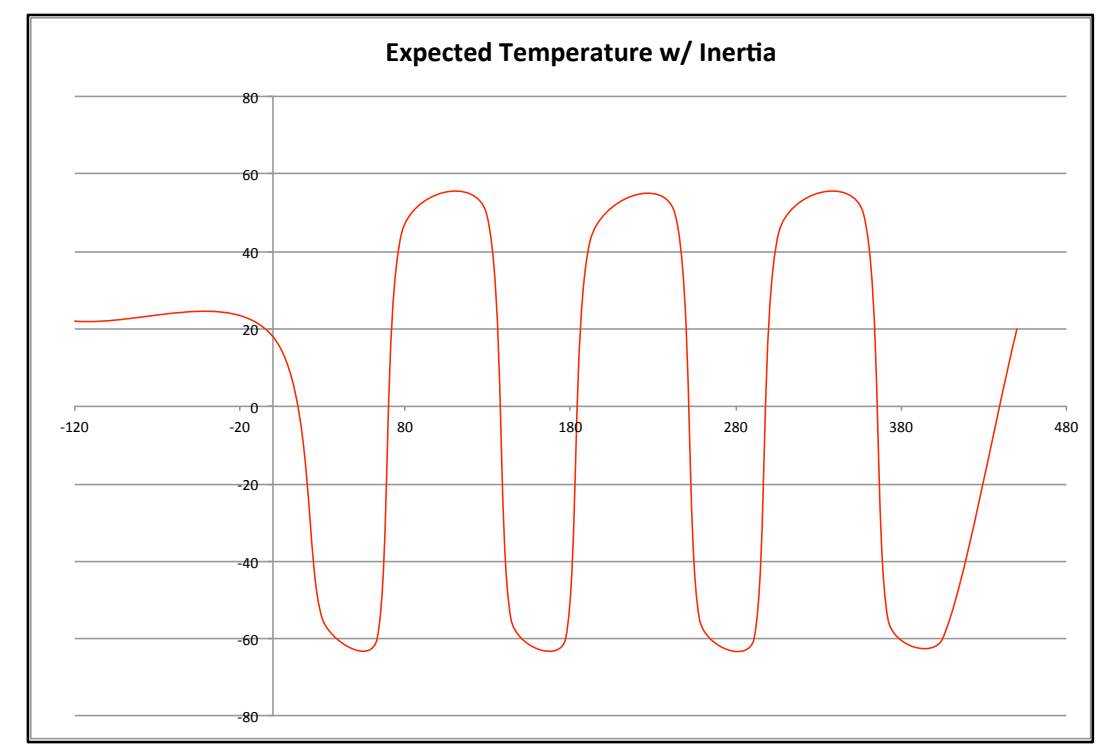

Figure 71. Expected Environment (Thermal Shroud) Temperature with Inertia Effects

Using the cold-biased thermal profile, each test profile was standardized to provide for four cold-ramps and three hot-ramps with one final "warm" ramp to return to a safe ambient temperature. While only representative of slightly more 
than three orbits, this was found to be a reasonable test plan. This put the entire testing time, including lab set-up, at an estimated 11 hours with no complications. Since this test needed to be performed multiple times, with limited help, on a limited schedule and with limited liquid nitrogen costs, this profile initially met the need. After the first two tests this profile was found to actually require 12 hours and was adjusted for the remaining two tests $(4 a, b$, and $c$ were combined into one test). First a baseline test with the satellite performing nominal operations was executed with the resulting output below. The key items to note are the severe thermal lag between the expected "environment" and the response of the satellite. Partly due to the thermal mass of the chamber itself and also due to the limitations inherent in heating and cooling a thermal shroud as quickly as possible. Still the simulation, when compared to the SINDA/TSS output, was found to be very comparable. 


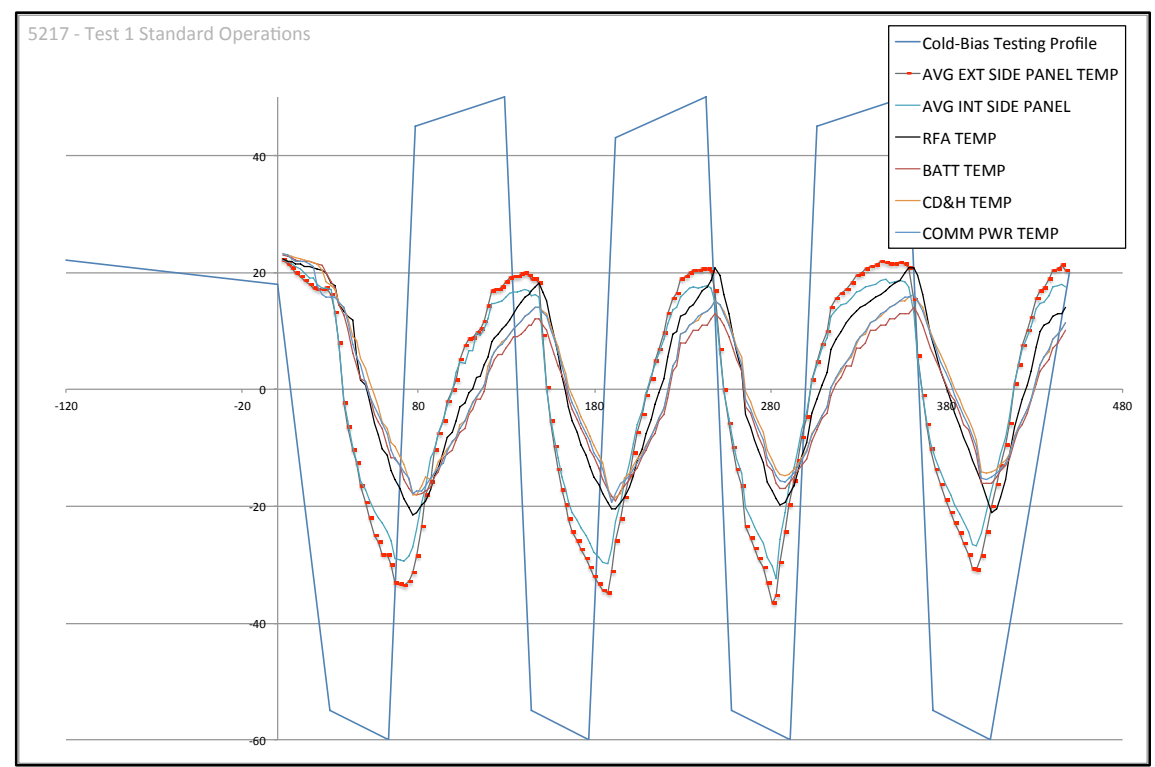

Figure 72. Standard Operations "Mode 1" Cycle Temperatures

This test was meant to establish the baseline four-cycle test and with which to confirm or verify the success criteria. While the initial baseline did not line up perfectly with the expected cold bias testing profile it was still a good calibration run and provided several lessons learned that would have to be used in subsequent tests.

The first lesson learned was that since the controller can not have both the solenoid and the heater circuit attached at same time there was at least a four to five minute lag between stopping the "ramp" temperature and initiating the next opposing "ramp." Moving to a hot ramp the temperature would be raised above the current cold controller temperature, the operator would note the time quickly, carefully walk along the side-scaffolding around the chamber, remove the solenoid liquid nitrogen electrical NEMA 5-20R connector and after checking, install the heater circuit NEMA 5-20R connector. These connections can be seen 
below in figure 73 where the white cabling (disconnected) supplies the heater current and the black cabling (connected) powers the solenoid valve. Also shown below is the location of the solenoid valve and the liquid nitrogen piping entering the chamber.

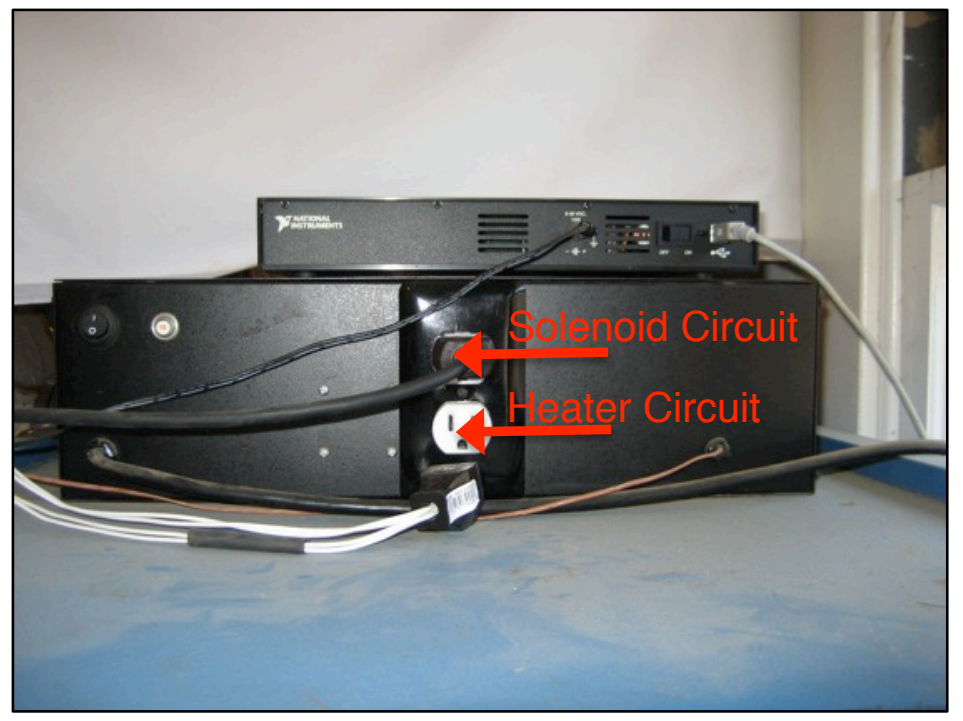

Figure 73. Omega Controller with National Instruments DAQ

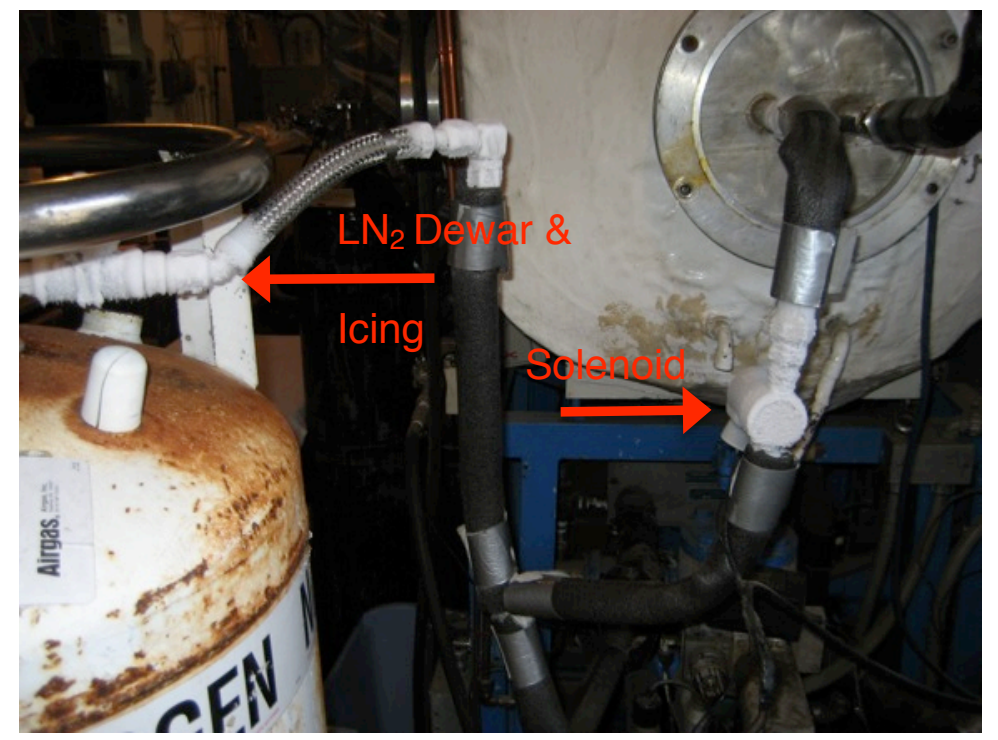

Figure 74 Solenoid Valve \& Liquid Nitrogen Dewar during Testing

Then the operator would walk back around the chamber and begin to 
increase the temperature, taking note of the ramp settings and associated times. For every cold ramp the operator would slow the ramp temperature beginning around $50^{\circ} \mathrm{C}$ ensuring that the heaters wouldn't peak beyond the test profile and then perform a similar process as was done for the hot ramp but instead removing the heater circuit and re-installing the solenoid circuit. This actually caused some noticeable leveling at the hot test point and was considered for future tests to be overly conservative as the heater overshoot was still within safe ranges. All of the ramp procedures were done while carefully noting the times and the ramp settings for future tests.

The author had not initially expected, nor accounted for, the added time delay between hot and cold settings. Since the four to five minute changeover was within the additional cold bias timeline the author evaluated it and found it to be negligible. Thus it did not force a redesign of the test criteria or a re-test of mode 1. Also during this test, the first ramp performed had two points where the ramp was slowed due to safety concerns. As the final cold temperature still reflected well against the other cycles and the first cycle was not found to be the minimum condition this inconsistency was considered negligible as well. All subsequent tests performed, except where specifically noted, used ramp times that endeavored to follow this initial baseline without the two point excursion as precisely as possible and thus mimic the exact same heating conditions. Using precise ramp settings allows for a better mode to mode comparison as changes in the temperature curves are attributable to the mode itself instead of being due 
to a change in ramp values or timing issues.

With this reference test established, post vacuum chamber shutdown, the next step was to check the satellite post-testing for any mechanical issues, contamination or otherwise. Once completed the operator and support personnel re-taped the satellite's antennae and placed it back into its container. Also, per the T-VAC standard operating procedure, the chamber was inspected for any mechanical or contamination issues post-test. This closeout process was repeated for each test performed and the satellite examination by the operators is shown in figure 75 .

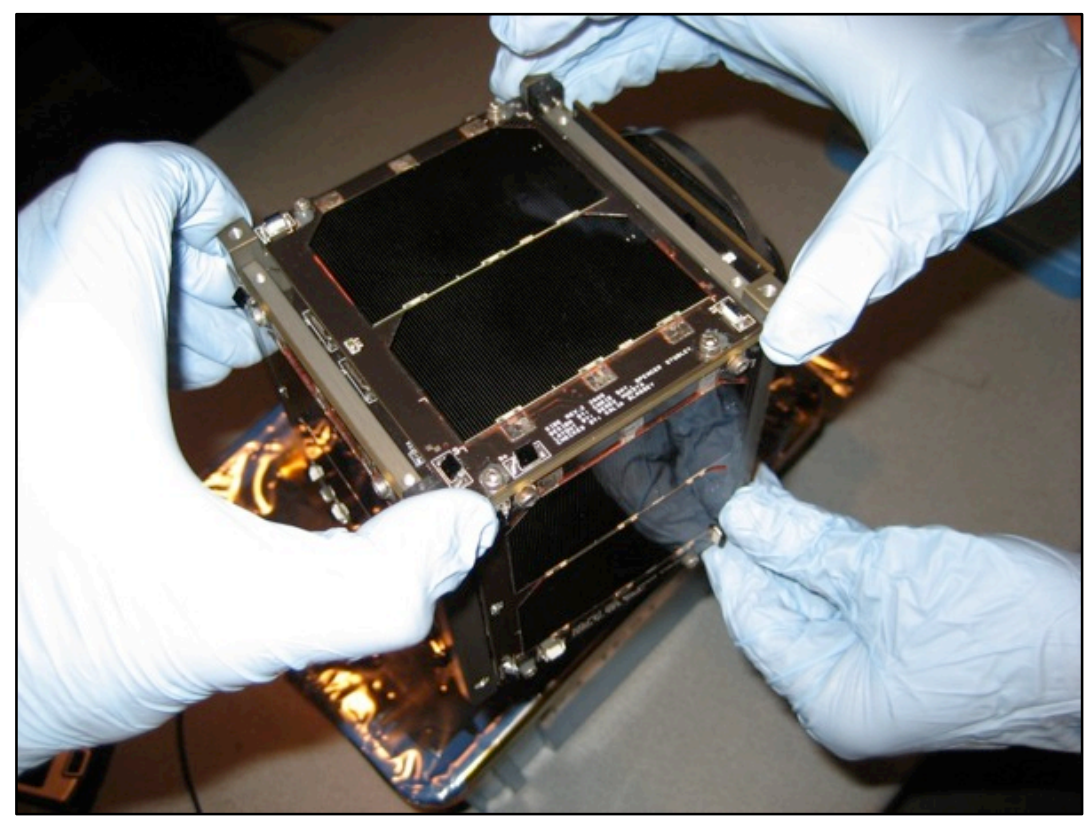

Figure 75. Post Test Inspection \& Antennae Tape-Down

The next test to be performed was the mode $2 \mathrm{a}$ increased beacon rate. The same procedures discussed for mode 1 thermal vacuum chamber testing were used for this test and the same ramp profile was used with the exception of 
the heater-safety issue mentioned above. By decreasing the overshoot expectations on the hot side the test was able to more accurately mimic on-orbit temperature swings and the thermal shock that the spacecraft is actually being exposed to. It also allowed for a shorter duration at the peak heating condition, also adding to the accuracy of the test environment. The cold cycle ramps were retained.

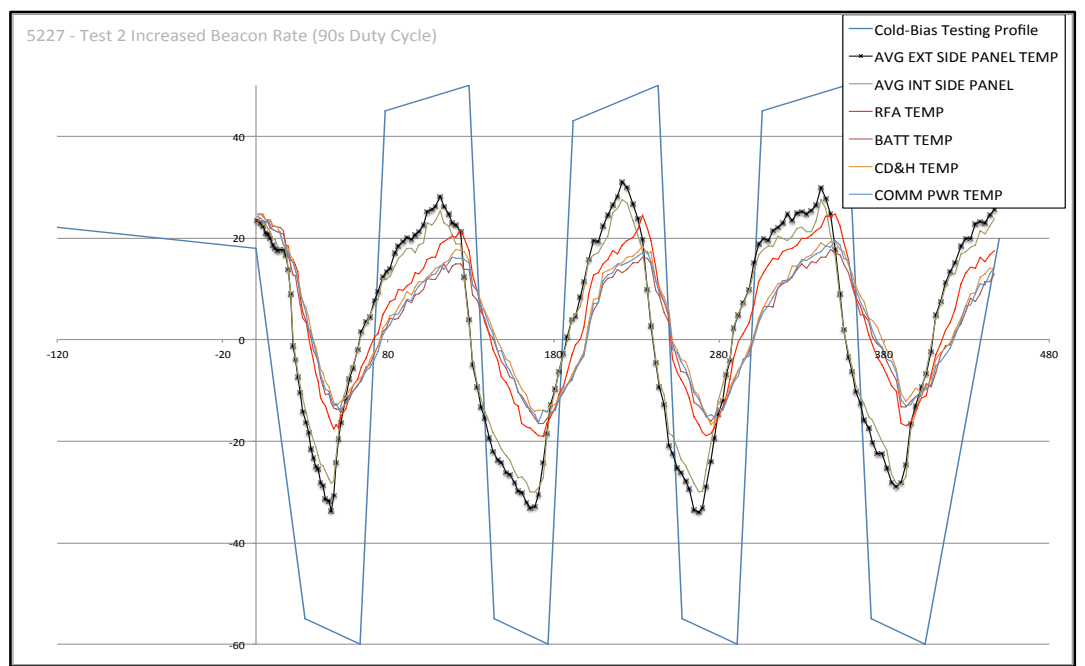

Figure 76. Mode 2a Increased Beacon Rate 90s

With the increased beacon rate at 90 s test completed, the next test of mode $2 \mathrm{~b}$ was prepared and executed. This test was coordinated beforehand to ensure that the 60 s beacon rate would not interfere with any other tests or operations being performed.

During the testing for mode $2 \mathrm{~b}$, on the second cold ramp cycle the timer being used was not properly set for the short increments and the cold ramp was extended, causing a slower ramp gradient but with little effect on the overall cold temperature. This excursion caused the entire mode $2 \mathrm{~b}$ test to run thirty minutes 
longer than the mode $2 \mathrm{a}$ and mode 1 tests. By extending the test, the next two ramps were done appropriately with the correct ramp gradients and timing. Overall it had no noticeable effect on the minimum temperature experienced during the test, just on the total test duration. This mode was found, post-testing, to be a noticeable improvement over the baseline (results in table 19).

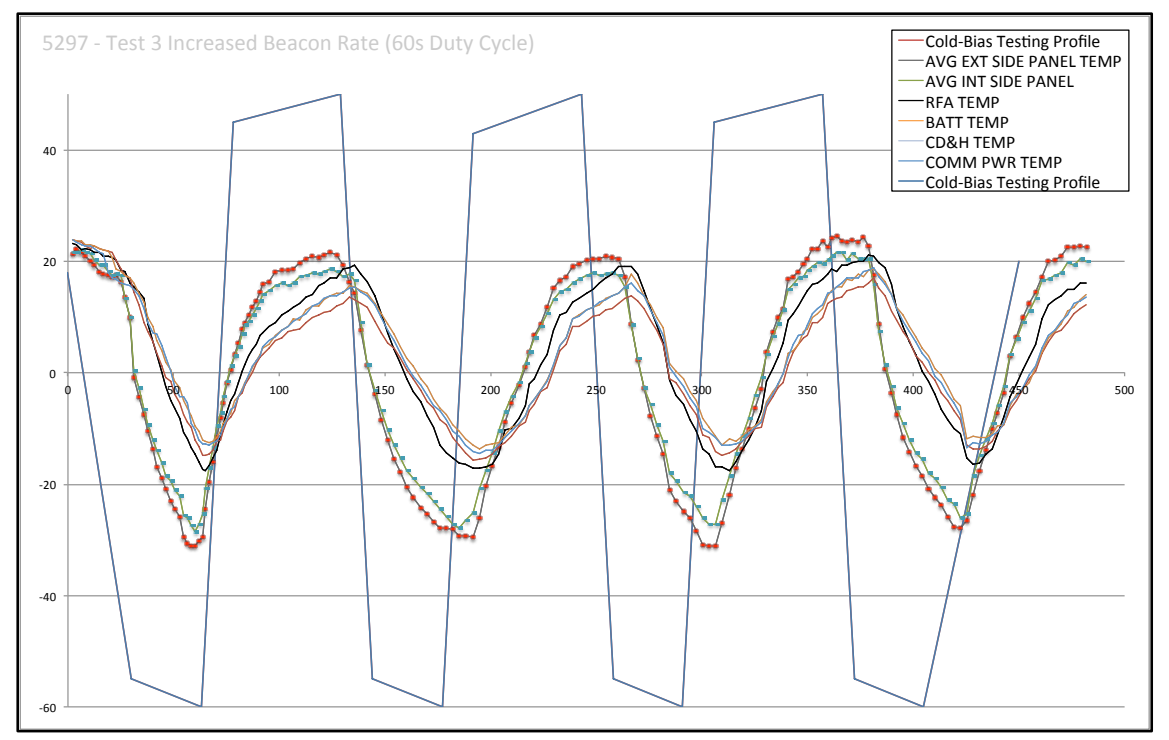

Figure 77. Mode 2b Increased Beacon Rate 60s

The mode 3 payload enabled test is shown below. For reference, this mode was calculated to use $1.779 \mathrm{~W}$ and was expected to have a slightly improved temperature versus the baseline and both modes previously tested. This test was started late due to other work being performed at the time. Initially the resistive heater circuit was connected, causing some initial heating and a slower first cold ramp. Also coordination challenges between the ground station and the chamber location occurred during the second cold ramp. The assisting operator was unsure of the ramp time intervals, causing some delay. 
In addition to challenges in ramp temperatures and the late start a conflict with other schoolwork at the time came up and the assistant supporting the test had to leave earlier than planned. For safety purposes thermal vacuum testing cannot be done with only one operator and so the author had to cut the test short by about ninety minutes or one full cycle. As the amount of increased heating in mode 3 was calculated to be only one tenth of a watt greater than the mode $2 b$ this decision was justified and the data collected during the test executed was considered to be acceptable to measure this mode. The results in table 19 show that this mode, while an improvement over the mode 1 baseline, was not in-line with the expected heating.

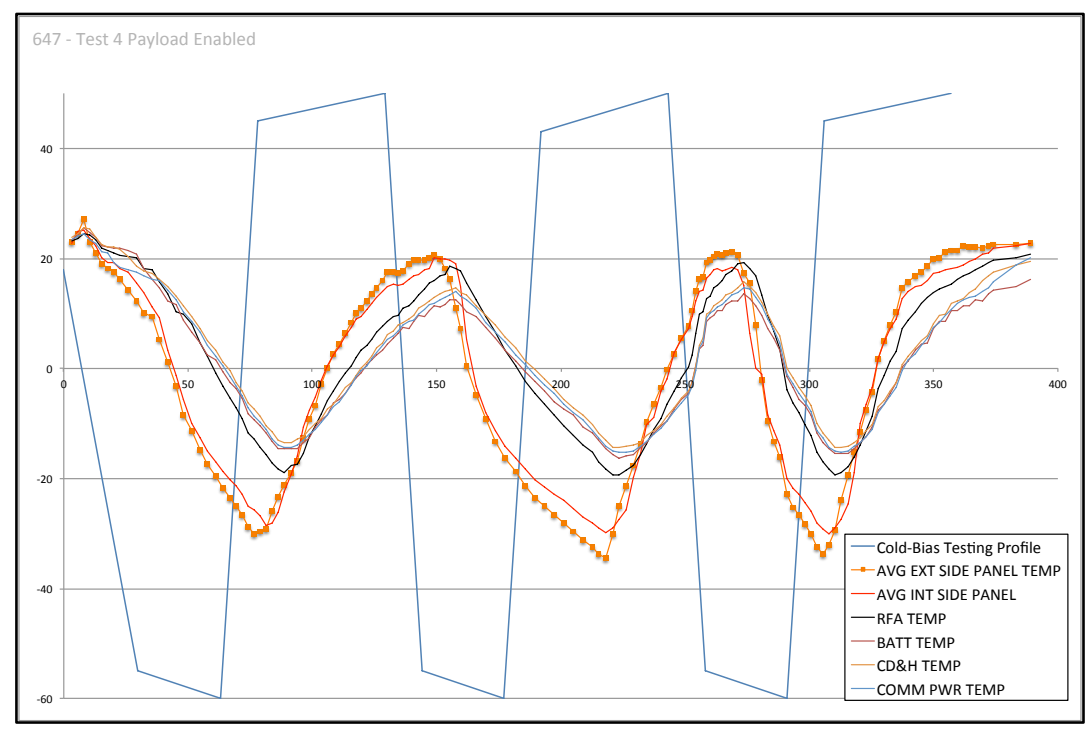

Figure 78. Mode 3 Payload Enabled Test

Mode 3 actually had less overall effect on the key temperature parameters than did Mode 2b. This was postulated to be due to the actual location of the heating in question being on the payload boards and only a slight increase in the battery 
heat generated due to $I^{2} R$ losses from that additional payload power. There was noticeable improvement over the baseline mode 1 but still not enough to qualify this as an effective mode for heating the satellite. Since the payload current draw is highly irregular, it may be that the actual duty cycle is much longer than the predicted duty cycle thus causing the total heat generation to be lower. As the baseline being used was from testing by the two electrical team leads for CP3 the power draw had to be considered an accurate estimation, but improvements in the total current draw and duty cycle measurements would definitively explain why this mode appeared to be placing less heat into the system than other "lower" power modes. The uncertainties caused by the shortened and altered testing parameters also cannot be overlooked as a potential source of error in the outcome of this test. For the reasons mentioned above (minimal increased heating load over mode $2 \mathrm{~b}$ and questionable duty cycle predictions) this mode was not re-tested.

To test mode 4 accurately a few initial assumptions had to be made. The high current required to implement $A D C$ modes can easily brown-out the satellite. Since this would cause serious issues for both testing and true utility of the mode on orbit the commanding was adjusted to a reasonable $50 \%$ of each mux's duty cycle, beginning with torquers on approximately ten minutes into the cold ramp and off approximately ten minutes before the hot ramp began. This again adjusted the total usage to a level consistent with the estimated power usage for ADC and in-line with the analyses done previously via other methods. Also mode 
4c was not tested due to concerns with browning-out or over-stressing the testarticle. By comparing the T-VAC results for modes $4 \mathrm{a}$ and $4 \mathrm{~b}$ with the analytical method results for correlation it was assumed that a correlation for Mode 4c could also be derived without putting hardware at risk. So modes $4 \mathrm{a}$ and $4 \mathrm{~b}$ were tested with mode 4 a being tested during the first and second cycles only. Then a return to mode 1 was allowed during the third cycle. Finally, mode $4 \mathrm{~b}$ was tested during the fourth and fifth cycle. Mode 1 was used during the third cycle for two reasons. First it was intended to "reset" the temperature condition of the satellite so that two modes, $4 \mathrm{a}$ and $4 \mathrm{~b}$, could be examined separately in one thermal vacuum test. Second it was intended to display the effect of multiple modes in one test while inspecting for any thermal inertia effects visible in the system.

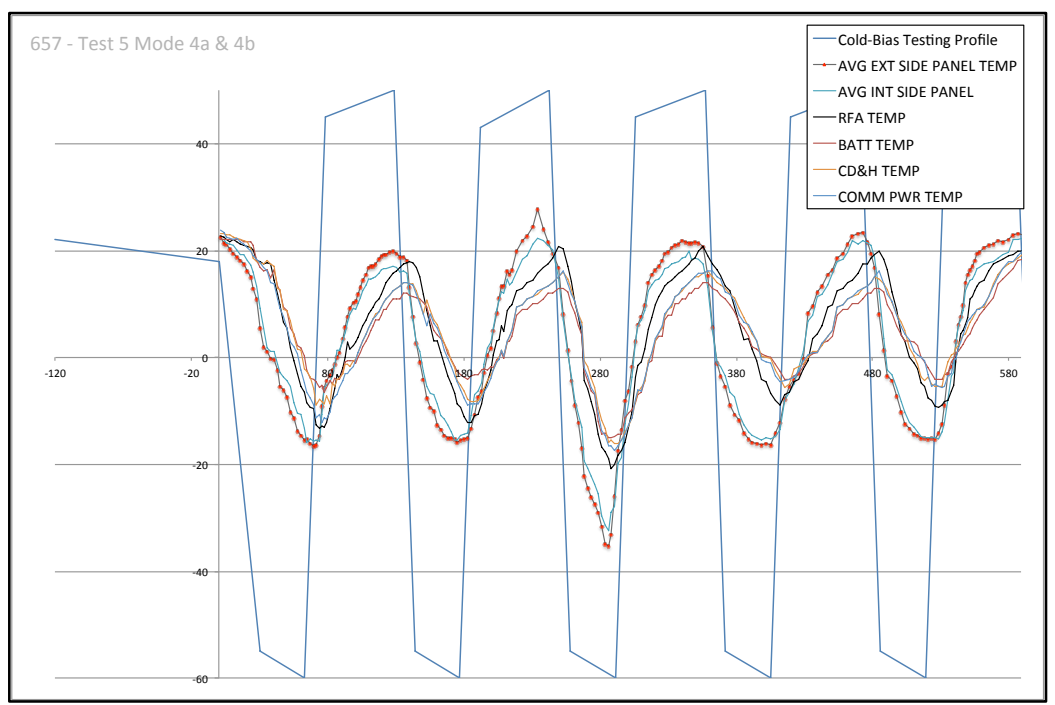

Figure 79. Combined Mode 4a, 4b and Mode 1 Insert Test 
During the hot ramp leading to the second cold cycle approximately four hours into active testing the heaters were ramped higher than the previously noted ramp profile called for to test the thermal inertia of the system during the "mode 1" section of the test. This caused spiking on the external side temperatures and some minor spiking on the internal temperatures but not as great of an effect as was expected. As the third cycle was set as a "mode 1" cycle this hot spike did not directly affect the other cycles, excepting a slight rise in the temperature on the RF amp and Comm power. The results in table 19 for modes $4 \mathrm{a}$ and $4 \mathrm{~b}$ show strong gradient reductions, well above the required verification criterion. As can be seen in figure 79 , above, the internal temperatures benefitted significantly from having both the ADC system engaged along with an increased beacon rate. With just the ADC alone engaged (mode $4 a$ ) the battery temperature delta from hot to cold was reduced by $32.5 \%$. The side panels also saw significant heating, reducing their exterior and interior delta temperatures by $35.4 \%$ and $31.7 \%$ respectively. With both the ADC and beacon rate increase (mode $4 \mathrm{~b}$ ) the battery temperature delta was reduced by $38.1 \%$ and the RF amp temperature delta saw a $29.4 \%$ decrease. The increased beacon rate made almost no impact on the exterior and interior side panel temperatures with decreases of $.4 \%$ and $.7 \%$ respectively. This is probably due to the increased heating from RF transmission being on an interior board with low view factor and a fairly low overall increase in the heat being generated. 
The next mode tested was the increased "data drop." This mode utilizes an increase in the contacts (ACK) with the satellite and increasing the regularity of "data dumps" which cause a large amount of processing and communication subsystem heating. The thermal vacuum test used the duty cycle established for section 3.3.4 for consistency. Figure 80 shows that mode 5 displayed improvements in several key parameters.

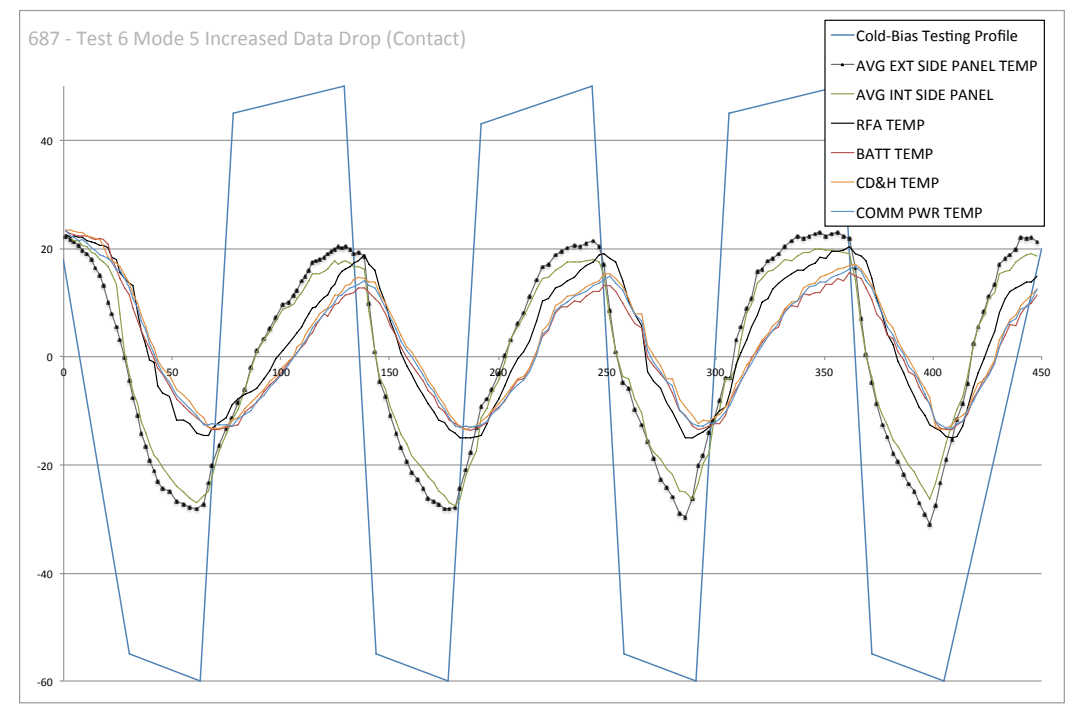

Figure 80. Mode 5 Increased Data Drop (Contact) Test

The increased contact mode 5 had only minimal effect (10.3\%) on the external temperatures, slightly better than mode $2 b(9.7 \%)$. The increase on the internal temperatures for key components was more significant. RFA temperature deltas were reduced by $15.4 \%$, battery deltas by $14.1 \%$, C\&DH temperatures by $14.5 \%$ and Comm power deltas by $15.7 \%$. The RF and Comm decreases met the necessary verification criteria and the C\&DH and battery temps were close enough that, taking into consideration the cold-biasing of the 
test, these too met the intent of the verification criteria.

The summary of all the testing temperature minimums, by mode, is presented below in Table 19. This compares the reduction to the temperature delta for the baseline on that component or surface using $+20^{\circ} \mathrm{C}$ as the "hot" condition temperature. Overall the modes tested compared favorably to the baseline condition and the cold condition minima's were increased for every mode tested.

Table 19. Thermal Vacuum Chamber Test Results

\begin{tabular}{|c|c|c|c|c|c|c|}
\hline MINIMUM & AVG SIDE PANEL EXT & AVG SIDE PANEL INT & RFA TEMP & BATT TEMP & CDH Temp & COMM PWR TEMP \\
\hline BASELINE & -36.7 & -32.4 & -21.5 & -19.1 & -19.2 & -19.4 \\
\hline$\%$ Difference & 0 & 0 & 0 & 0 & 0 & 0 \\
\hline $90 \mathrm{~s}$ & -34.02 & -30 & -19.05 & -16.6 & -16.8 & -16.3 \\
\hline \% Difference & $4.7 \%$ & $4.6 \%$ & $5.9 \%$ & $6.4 \%$ & $6.1 \%$ & $7.9 \%$ \\
\hline 60s & -31.2 & -28.49 & -17.7 & -15.7 & -13.7 & -14.5 \\
\hline \% Difference & $9.7 \%$ & $7.5 \%$ & $9.2 \%$ & $8.7 \%$ & $14.0 \%$ & $12.4 \%$ \\
\hline PAY ENABLED & -34.482 & -30.0708 & -19.35 & -16.72 & -14.45 & -15.54 \\
\hline \% Difference & $3.9 \%$ & $4.4 \%$ & $5.2 \%$ & $6.1 \%$ & $12.1 \%$ & $9.8 \%$ \\
\hline ADC ON & -16.6 & -15.8 & -13.2 & -6.4 & -8.85 & -12.2 \\
\hline$\%$ Difference & $35.4 \%$ & $31.7 \%$ & $20.0 \%$ & $32.5 \%$ & $26.4 \%$ & $18.3 \%$ \\
\hline$A D C$ ON $+90 s$ & -16.4 & -15.4 & -9.3 & -4.2 & -5.7 & -5.6 \\
\hline$\%$ Difference & $35.8 \%$ & $32.4 \%$ & $29.4 \%$ & $38.1 \%$ & $34.4 \%$ & $35.0 \%$ \\
\hline DATA DROP & -30.88 & -27.5624 & -15.1 & -13.6 & -13.5 & -13.2 \\
\hline$\%$ Difference & $10.3 \%$ & $9.2 \%$ & $15.4 \%$ & $14.1 \%$ & $14.5 \%$ & $15.7 \%$ \\
\hline
\end{tabular}

The summary presented in table 19 uses minimums over all cycles for each mode tested. The realized improvements were readily apparent in this table and it can be seen that three of the five modes tested $(3,4 a, 4 b)$ meet or exceed the verification criteria. Modes $2 \mathrm{a}$ and $2 \mathrm{~b}$ similarly make non-trivial improvements, especially in reducing the battery temperature total delta by $8.7 \%$ and $6.4 \%$ respectively. The increase of $8.7 \%$ on the battery delta by mode $2 \mathrm{~b}$ corresponds to an increase of around $115 \mathrm{mAh}$ or $6.9 \%$ on the total discharge capacity of the CGA103450A batteries. The $38.1 \%$ delta decrease from mode $4 \mathrm{~b}$ corresponds 
to an increase of around $235 \mathrm{mAh}$ or $14.2 \%$ of the total discharge capacity of the CGA103450A batteries.

As can be seen from the graphs and results, while there were challenges in maintaining the timeline, the heat ramps were accurately retained so that steps and the associated system temperature responses could be evaluated with each test's results and easily compared to one another.

Further discussion of these results and comparisons to the analytical and thermal models are shown in section 4.1, Review of Results Against Verification Criteria.

\subsection{On-Orbit Commanding of Modes 1, 2a and 5}

During the late stages of this thesis, on April $17^{\text {th }} 2007$, the first successful DNEPR CubeSat launch took place. The author was privileged to be one of the operators during the initial two months of on orbit operations. It was during this time that the first anomaly resolutions occurred and a few limited opportunities arose for on-orbit testing of this thesis. Since communication with the satellites was restricted and challenges with contacting CP3 were numerous, the amount of testing performed was constrained to limited tests of only mode $2 a$ and mode

5. These modes could be tested on CP2 rather early because they complied with the team's CONOPs at the time, and CP3 only later once a stable command link had been established. The graphs below in figure 83 show a snapshot of the onorbit thermal response of the CP3 side panels after one month on orbit. 


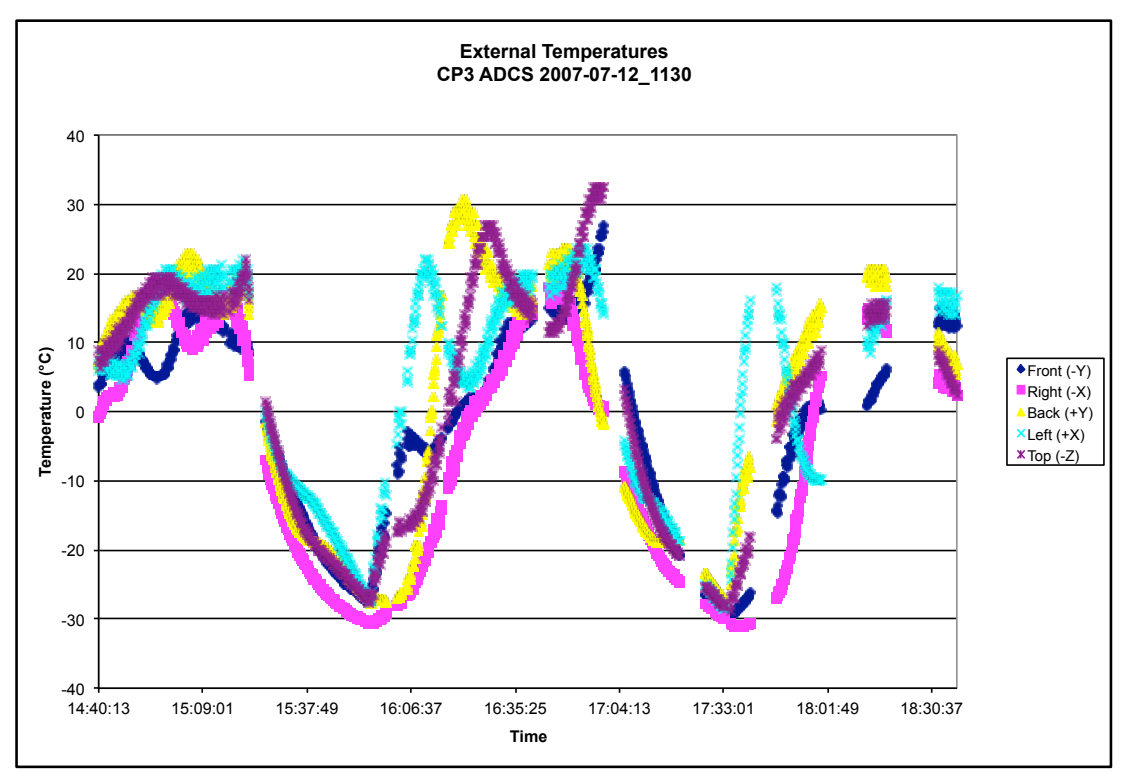

Figure 81. External On-Orbit Temperatures 1, CP3

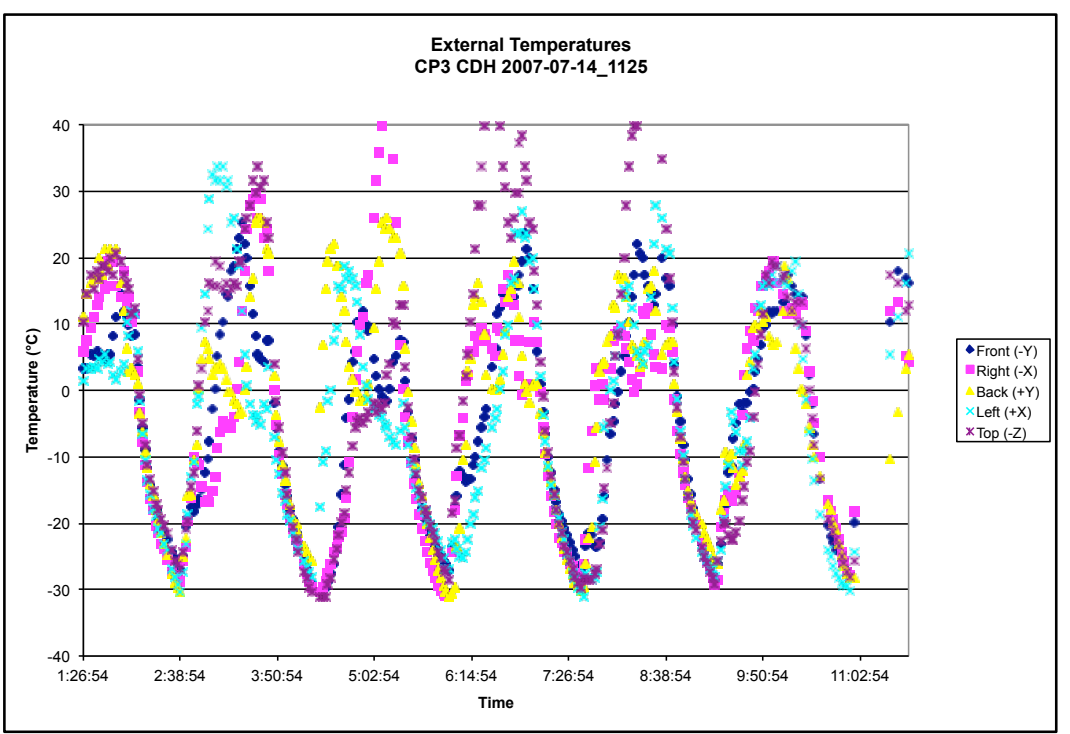

Figure 82. External On-Orbit Temperatures 2, CP-3 


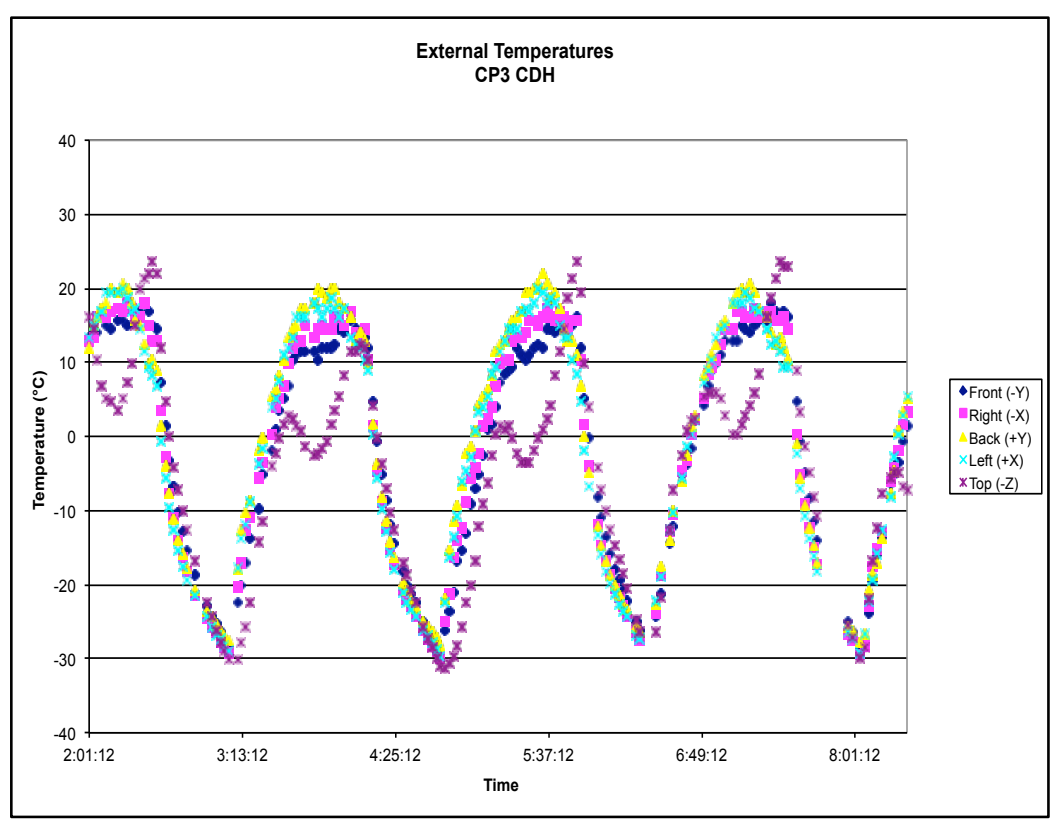

Figure 83. Initial On-Orbit Temperatures External 1

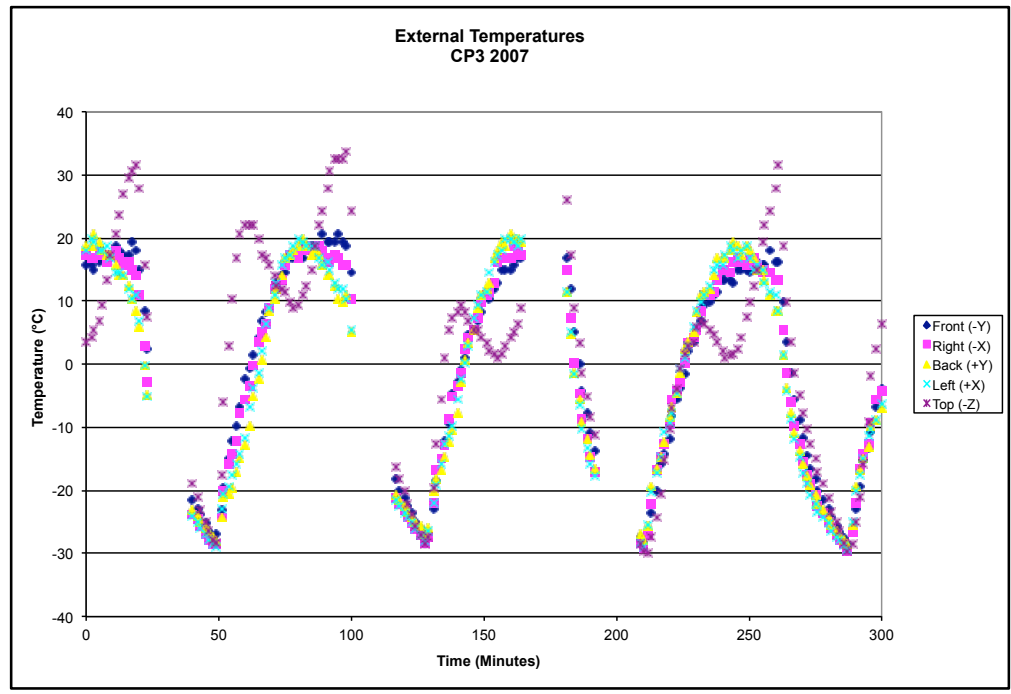

Figure 84. Initial On-Orbit Temperatures External 2

Using the SRI dish to overcome receive sensitivity issues on May $24^{\text {th }}$ 2007, two successful sensor dump operations were performed on CP2, raising the internal temperatures on the $\mathrm{C} \& \mathrm{DH}$ temp by approximately $5^{\circ} \mathrm{C}$ on the sunlit side of the orbit and $2^{\circ} \mathrm{C}$ on the umbra side while also raising the RFA temp $2^{\circ} \mathrm{C}$ 
on the umbra side of the orbit. Also, the beacon rate was set to 120 seconds vice the 300 seconds that it was initially at. This was the first indication that data dump (mode 5) could be useful on orbit. CP3 still could not be reached, even using the SRI 20m dish, and the operations team postulated this was due to the extremely low amount of power being utilized on CP3 versus CP2 making the receive sensitivity on CP3 even lower than that of CP2 because it had no thermal damping effect from internal heat generation. Contacting CP2 became easier after this adjustment was made, both because more regular beacons were easier to track manually and also because the additional on-board heating being generated.

On June $11^{\text {th }} 2007$, CP2 was tested in mode $2 b$. The two pass data responses showed an increase of $3^{\circ} \mathrm{C}$ in the RF temperature and an increase of $4^{\circ} \mathrm{C}$ on the Comm processor. In addition communication acknowledgement from the satellite on the subsequent passes was improved, despite being lower on the horizon and thus further from the ground station. The RSSI improved from 53 to 4F corresponding to a 4 point decrease ( 83 to $79-95 \mathrm{dbM}$ ). This was in line with the results from thermal vacuum testing.

On July $14^{\text {th }} 2007$, CP3 underwent partial testing of mode 5 with several successful downlinks and two data dumps completed. As expected, the internal temperature of the satellite rose. However, the two completed passes were daytime passes and as such were successful only in increasing the rise of the internal temperatures and had almost no effect on the cold pass. As a 
comparison illustrating the difference in internal temperatures for both satellites once in "normal ops" the graph below (figure 85) shows four CP2 orbits on the left and four CP3 orbits on the right. It should be noted that the CP2 internal temperatures are consistently $3-7^{\circ} \mathrm{C}$ higher during the umbra than the equivalent CP3 temperature. This is due to the increased heat generation from both increased beacon activity and increased processing activity on CP2. The sunlit portions compare almost evenly between the two satellites with CP2 having significantly more instability on the external panels but very similar temperature profiles internally.

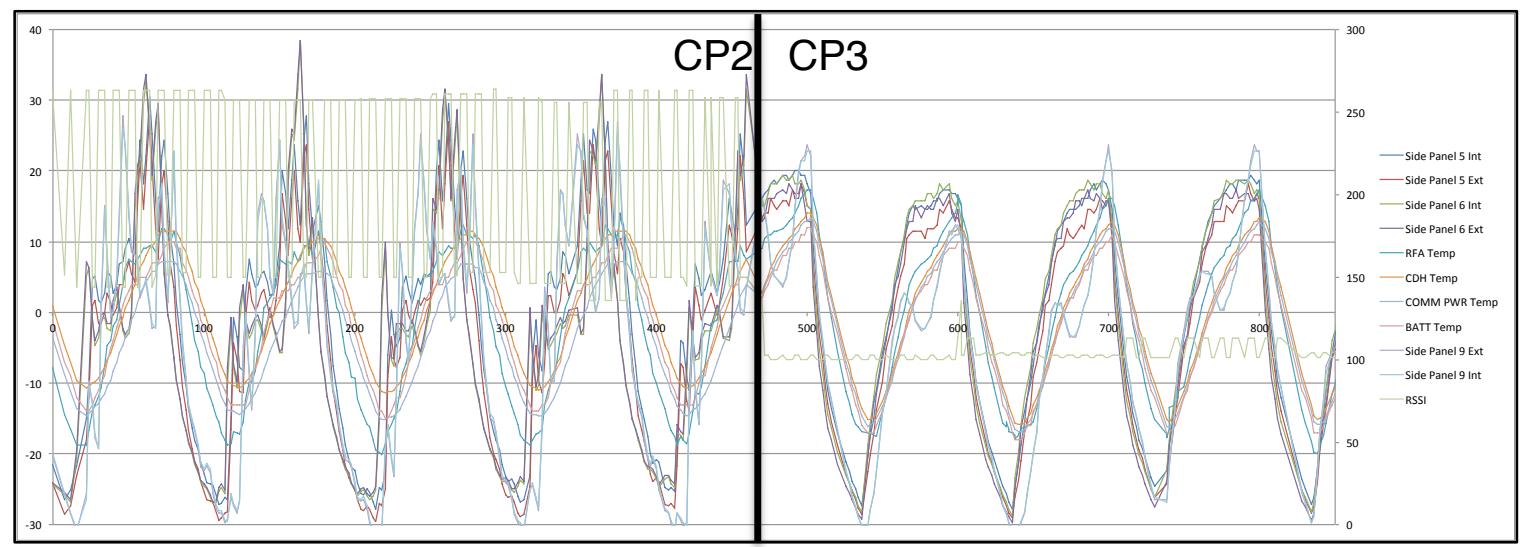

Figure 85. CP2 vs CP3 On-Orbit Temperature Comparison 


\subsection{Thesis Results and Conclusions}

\subsection{Review of Results Against Verification Criteria}

The results obtained from the analytical method, two unique modeling methods, thermal vacuum testing and finally the limited on-orbit testing are summarized in this section. The conclusion reached after all of these analyses, modeling and testing were completed, is that a 1-U CubeSat can improve its thermal condition utilizing existing hardware and software. A table summarizing the verification criteria established in section 2.3.3 is shown below for reference (table 20).

Table 20. Verification Criteria Compilation

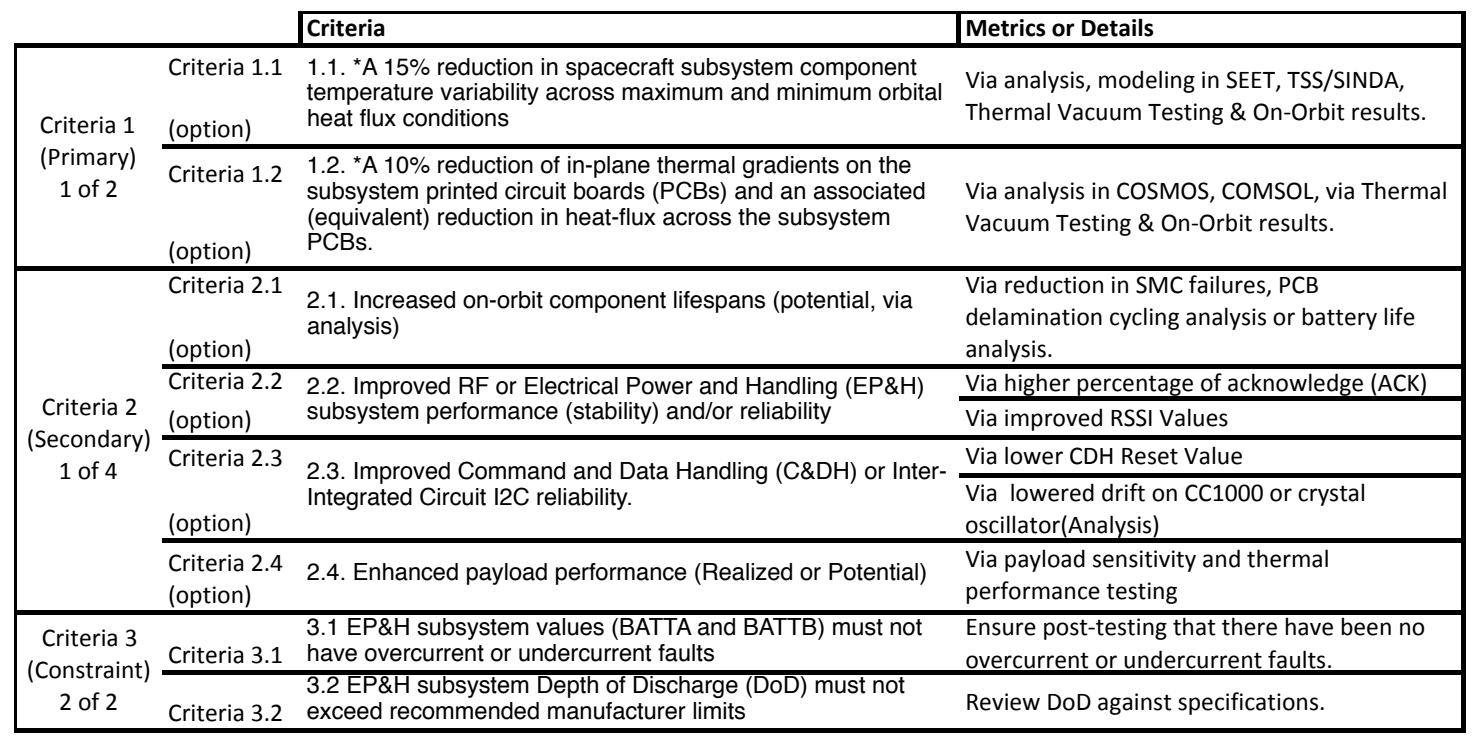

The first method used to generate verification evidence was analytical calculations using the thermal balance equation for a CubeSat. The initial analytical results indicated that the satellite could make significant reductions in its onboard temperature swings and specifically modes $2 b, 3,4 a, 4 b, 4 c$ and 5 had the capability to meet the verification criterion 1.1 . 
Table 21. Criteria 1.1 Verification Evidence; Percent Reduction Max to Min

\begin{tabular}{|l|c|c|c|}
\hline Verification Criteria 1.1 - 15\% Orbital Max-Min Reduction & Solved for T Kelvin & (Celsius) & $\%$ Reduction \\
\hline CASE 2b - Cold w/ Adjustments & 241 & -32 & \\
\hline CASE 5 - Hot w/ Adjustments & 346 & 73 & \\
\hline CASE 3 - Mode 1 (Standard Operations) & 241 & -32 & $0.0 \%$ \\
\hline CASE 3 - Mode 2a. (Inc. Beacon Rate - 90s) & 253 & -20 & $11.3 \%$ \\
\hline CASE 3 - Mode 2b. (Inc. Beacon Rate - 60s) & 258 & -15 & $15.6 \%$ \\
\hline CASE 3 - Mode 3. (Payload Enabled - ON) & 258 & -15 & $16.2 \%$ \\
\hline CASE 3 - Mode 4a. (ADC ON) & 287 & 14 & $43.4 \%$ \\
\hline CASE 3 - Mode 4b. (ADC ON \& Beacon Inc. - 90s) & 289 & 16 & $45.0 \%$ \\
\hline CASE 3 - Mode 4c. (ADC ON \& Beacon Inc. - 60s) & 292 & 19 & $47.9 \%$ \\
\hline CASE 3 - Mode 5. (Increased "Data Drop") & 273 & 0 & $30.5 \%$ \\
\hline
\end{tabular}

The next step was using the SEET modeling tool based in STK to model each scenario. The SEET tool generated large deltas, in family with the analytical method, but was still able to accurately model the resulting improvement from each mode. Using the SEET function modes $4 a, 4 b, 4 c$ and 5 were all found to meet the verification Criterion $1.115 \%$ reduction. Mode 3 was close to but not quite able to meet the criteria in this analysis. While the total reduction may not have met the criterion for the first three modes, both the SEET analysis and the SINDA/TSS analysis (table 22 below) assumes a single node for the satellite temperature. This means that those modes could still be proven via other methods to be effective at reducing the key temperature parameters, but for a lumped mass analysis via these methods they could not.

Table 22. SINDA Output Results Summary by Mode

\begin{tabular}{|r|r|r|r|r|r|r|r|r|}
\hline & Mode 1 & Mode2a & Mode 2b & Mode 3 & Mode 4a & Mode 4b & Mode 4c & Mode 5 \\
\hline Min(F) & 1.2 & 3.2 & 5.7 & 6.3 & 24.1 & 26.2 & 27.8 & 15.6 \\
\hline Min (C) & -17.1 & -16.0 & -14.6 & -14.3 & -4.4 & -3.2 & -2.3 & -9.1 \\
\hline \% Reduction & $0.0 \%$ & $3.0 \%$ & $6.7 \%$ & $7.6 \%$ & $34.3 \%$ & $37.4 \%$ & $39.8 \%$ & $21.6 \%$ \\
\hline
\end{tabular}


The satellite and orbit were modeled in TSS and solved in SINDA with the different heating modes more accurately portrayed as step functions (SEET was unable to model step functions). The results showed that modes $4 \mathrm{a}, 4 \mathrm{~b}, 4 \mathrm{c}$ and 5 were all predicted to meet the verification criterion 1.1 reduction of $15 \%$ in onorbit temperature differences.

The next method of reviewing the thesis against the verification criteria was via thermal vacuum testing. Table 23 summarizes the results of the thermal vacuum chamber tests. Modes $4 \mathrm{a}, 4 \mathrm{~b}$ and 5 were all able to meet the verification criteria (mode 5 for key parameters only). Mode $2 \mathrm{~b}$ was also found to have significant improvement in both RFA temperatures and C\&DH temperatures. With the inherent conservatism of the test there is reason enough to justify considering mode $2 \mathrm{~b}$ to have met the criteria for those key parameters.

Table 23. Criteria 1.1 Thermal Vacuum Chamber Test Results

\begin{tabular}{|c|c|c|c|c|c|c|}
\hline MINIMUM & AVG SIDE PANEL EXT & AVG SIDE PANEL INT & RFA TEMP & BATT TEMP & CDH Temp & COMM PWR TEMP \\
\hline BASELINE & -36.7 & -32.4 & -21.5 & -19.1 & -19.2 & -19.4 \\
\hline$\%$ Difference & 0 & 0 & 0 & 0 & 0 & 0 \\
\hline $90 \mathrm{~s}$ & -34.02 & -30 & -19.05 & -16.6 & -16.8 & -16.3 \\
\hline$\%$ Difference & $4.7 \%$ & $4.6 \%$ & $5.9 \%$ & $6.4 \%$ & $6.1 \%$ & $7.9 \%$ \\
\hline $60 \mathrm{~s}$ & -31.2 & -28.49 & -17.7 & -15.7 & -13.7 & -14.5 \\
\hline$\%$ Difference & $9.7 \%$ & $7.5 \%$ & $9.2 \%$ & $8.7 \%$ & $14.0 \%$ & $12.4 \%$ \\
\hline PAY ENABLED & -34.482 & -30.0708 & -19.35 & -16.72 & -14.45 & -15.54 \\
\hline$\%$ Difference & $3.9 \%$ & $4.4 \%$ & $5.2 \%$ & $6.1 \%$ & $12.1 \%$ & $9.8 \%$ \\
\hline ADC ON & -16.6 & -15.8 & -13.2 & -6.4 & -8.85 & -12.2 \\
\hline$\%$ Difference & $35.4 \%$ & $31.7 \%$ & $20.0 \%$ & $32.5 \%$ & $26.4 \%$ & $18.3 \%$ \\
\hline$A D C O N+90 s$ & -16.4 & -15.4 & -9.3 & -4.2 & -5.7 & -5.6 \\
\hline$\%$ Difference & $35.8 \%$ & $32.4 \%$ & $29.4 \%$ & $38.1 \%$ & $34.4 \%$ & $35.0 \%$ \\
\hline DATA DROP & -30.88 & -27.5624 & -15.1 & -13.6 & -13.5 & -13.2 \\
\hline$\%$ Difference & $10.3 \%$ & $9.2 \%$ & $15.4 \%$ & $14.1 \%$ & $14.5 \%$ & $15.7 \%$ \\
\hline
\end{tabular}

The primary criterion 1 was met through the testing previously discussed.

Now using the analyses and test data obtained, the remainder of the criteria could be verified. 
Using the data obtained from the COSMOS simulation on in-plane temperatures and heating for each mode, it was found that the C\&DH board, specifically the PIC18 processor, had a $21 \%$ lower gradient between itself and the PCB substrate. When the hypothesis first took form it was developed to allow success through multiple paths since "improvement" was a difficult parameter to quantify and the testing to be performed was not yet established. Since only one of the two primary constraints was required, the results shown above and the additional data from section 3.3.6 are sufficient to fulfill criterion 1.2.

The first of the four optional secondary verification criteria (2.1) was extending the operable lifetime of the satellite. Two specific failure modes; delamination of the PCB and solder joint cracking on the surface mount components described in Appendix $\mathrm{K}$ can be effectively reduced (increase \# cycles to failure) by the implementation of this thesis. While delamination is unlikely given the parameters and cycling in question, the cracking experienced by the surface mount components definitely could occur and the ability to reduce the panel temperatures by up to $35.8 \%$ would provide a reduction in the fatigue on those joints. In addition to the reduction in panel cycling the increase in internal temperature helps in limiting lithium metal plating on the anode during cold temperature charging.

Lithium ion batteries are typically not rated for charging below $0^{\circ} \mathrm{C}$ due to their anode plating phenomenon. Panasonic rates the battery in CP3 for charging exclusively between 0 and $45^{\circ} \mathrm{C}$. This creates a serious issue as the 
battery temperatures predicted through analysis $\left(-32^{\circ} \mathrm{C}\right)$, verified in thermal vacuum testing $\left(-19.1^{\circ} \mathrm{C}\right)$, and finally experienced on orbit $\left(-18.3^{\circ} \mathrm{C}\right)$, all are well below this rated limit $\left(0^{\circ} \mathrm{C}\right)$. At subzero temperatures lithium metal begins to plate on the anode of the batteries during charging. This plating is amplified by the charging amount as well, since the CP3 solar panels are most efficient at the coldest temperature and the coldest temperature for both the solar panels and the batteries is experienced immediately before leaving eclipse this has a compounding effect. Just as the battery is coldest the solar panels are attempting to charge it at the highest rate.

As the charging from the solar panel occurs the battery loses potential (shown in Appendix M, Figure 129). At $-10^{\circ} \mathrm{C}$ the potential is anywhere from $10 \%$ to $50 \%$ less than the equivalent battery charged at $0^{\circ} \mathrm{C}$. The cumulative effect of such low-temperature plating has been shown via testing to reduce the total capacity over equivalent cycles by $12.5 \%$ from $0^{\circ} \mathrm{C}$ to $-10^{\circ} \mathrm{C}$ and by $21.9 \%$ from $0^{\circ} \mathrm{C}$ to $-20^{\circ} \mathrm{C} \cdot{ }^{24}$ As the battery temperature during thermal vacuum testing was increased from a minimum of $-19.1^{\circ} \mathrm{C}$ down to a minimum of $-4.2^{\circ} \mathrm{C}$ this provides a significant improvement (10-20\% estimated) in the total capacity available over the lifetime of the battery by limiting the losses due to low temperature charge plating effects. The actual percentage improvement had to be estimated as the results from the study performed were non-linear and only six data points were presented for the capacity reduction.

Next on the list, criterion 2.2 called for an improved RF or EP\&H 
performance. The metric used to quantify this performance increase was either increased ACK or improved RSSI values. From the testing performed and summarized in Appendix L the RSSI values were found to trend strongly upwards during temperature ramps and cold soaks. They trended down during 'warm' periods and under smaller temperature ramps. Given that the testing and analysis shows that both smaller ramps and 'warmer' system temperatures can be achieved, the RSSI parameter for criterion 2.2 was verified. The on-orbit testing also supported this verification, though not to the same degree as the thermal vacuum testing did. During on orbit operations testing the same trends were observed for RSSI and system "ACK," as more heat was placed into the satellite via increased contacts \& increased beacon rates the sensitivity of the satellite showed improvement. ${ }^{A}$ The second metric of the satellite providing an "acknowledge" or "ACK" to commands sent was also tested during the RSSI tests (Appendix L) and break points were found both on the cold dwell and on the hot/cold and cold/hot ramps. This further evidenced that large gradients were at least part of the origin for receive sensitivity issues.

Criterion 2.3 was established to show improvement C\&DH or $\mathrm{I}^{2} \mathrm{C}$

A Since the on-orbit testing opportunities were so limited this was only considered an "observation" of the system and not evidence of an outcome 
performance and reliability. The best metric for this was found to be $\mathrm{C} \& \mathrm{DH}$ reset values and lowered drift on the oscillator crystal (as this directly impacts the $\mathrm{I}^{2} \mathrm{C}$ performance). The reset value was later found to be a poor metric as the software \& electrical design team did an excellent job in building a system that did not reset easily, or at least not due to thermal inputs. As no resets occurred during testing this metric was found to be unusable. The lowered drift rate metric however was easy to verify once the temperature increases were tested and quantified. Per the data in Appendix $\mathrm{M}$, the temperature variability of the $\mathrm{I}^{2} \mathrm{C}$ clock and the temperature improvements that were found to be possible the frequency stability could be decreased from 3.5ppm down to $2 \mathrm{ppm}$, an improvement of $75 \%$. In addition the trim register frequency per Least Significant Bit, which also could be reduced by $48 \%$ in total variability (from $28.4 \mathrm{ppb}$ to 14.6ppb). These improvements are considered to verify criterion 2.3.

The last criterion for verification was number 2.4 and reads "Enhanced Payload Performance - Realized or Potential." This was the most challenging of all the criteria, as it requires strong indication of improvements on a system that is not easily tested. Using data acquired during the author's support to a fifteen hour thermal vacuum cycle test performed for K. Glassey's thesis ${ }^{25}$ specifically addressing the payload on $\mathrm{CP} 3$, this criterion can be verified. Figure 86 below illustrates the payloads current draw over a cycle (with constant duty cycle on the payload). 


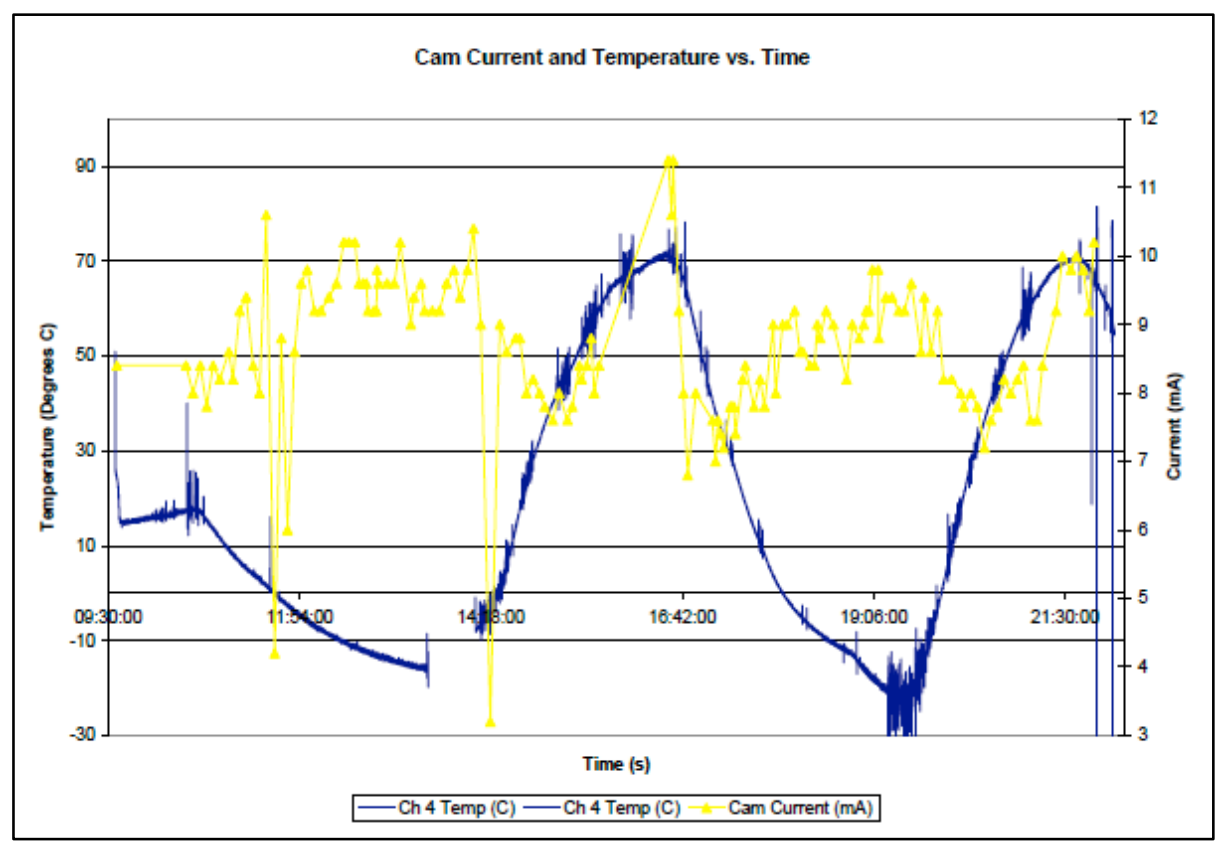

Figure 86. Payload Current Draw Across Temperature Spectrum ${ }^{25}$

The payload's current draw was greatly affected during cycle ramping, after review the only explanation that could be ascertained was that thermal strain on connections was increasing the resistance and causing the current to increase. In addition to the current increase the cameras were not rated to below $10^{\circ} \mathrm{C}$ and $-10^{\circ} \mathrm{C}$ for the KAC 9638 and 9648 respectively. During testing, as the payload temperature dropped below the rated levels sensitivity quickly degraded. At extremely low temperatures the CMOS would white out. The required black levels could either be manually adjusted to account for this issue, but over several orbits and with limited communication, manual adjustment was not reasonable for true operations. Thus the payload operations would definitively be improved with the application of the modes discussed and Criterion 2.4 was met. 
The last criteria were 'constraints' or items that were not goals to be met but instead limits that could not be broken, in order to consider the implementation successful. Constraint 3.1 called for EP\&H subsystem values for BATTA and BATTB to show no overcurrent or undercurrent faults due to the implementation of the modes. Although modes $4 \mathrm{a}, 4 \mathrm{~b}$ and $4 \mathrm{c}$ were the highest current draws of all the postulated and tested schemes, none of them caused an overcurrent fault during testing, showing that constraint 3.1 was met. Constraint 3.2 called for the EP\&H subsystem not to exceed the manufacturers recommended depth of discharge (DoD) limits. Based on the duty cycle and modes utilized the maximum DoD for the batteries was calculated to be $22.9 \%$ after one eclipse under the largest power draw. This is well within the manufacturer's recommended lifetime DoD limit specified (Appendix A). As this is within the DoD limit while taking into consideration charging only during the satellite's sunlit period the constraint was successfully met.

With all the verification criteria met, and based on the aforementioned evidence presented and tests performed, the conclusion can be made that adaptive component usage is a viable thermal management technique for CubeSats. The modes presented each have different applicability but each one demonstrates strong capability for improving the satellites thermal condition. Mode 5 presented the best system level response with a lower DoD than other modes while mode $4 a, 4 b$ and $4 c$ presented the greatest heating opportunity if DoD were not a factor. Even modes $2 \mathrm{a}$ and $2 \mathrm{~b}$, while providing only a small 
thermal improvement had secondary benefits as higher beacon rates also increase the ability of ground tracking systems and personnel to contact the satellite providing a better opportunity to implement mode 5 .

\subsection{Future Studies}

\subsubsection{Commanding}

One of the key enablers to making this form of thermal management a reality is having available commanding time. Because the most majority of these modes require some form of care and feeding, especially the high-power modes, the amount of available uplink time is critical. The figure below, taken from a separate CubeSat thesis regarding ground station automation routines, illustrates how non-steady commands can use changes in ground operations to enable them to still be a practical part of the adaptive component utilization thermal scheme. ${ }^{26}$ The increase from $2 \%$ to $67 \%$ command availability is very much a game changing item for the implementation of portions of this thesis.

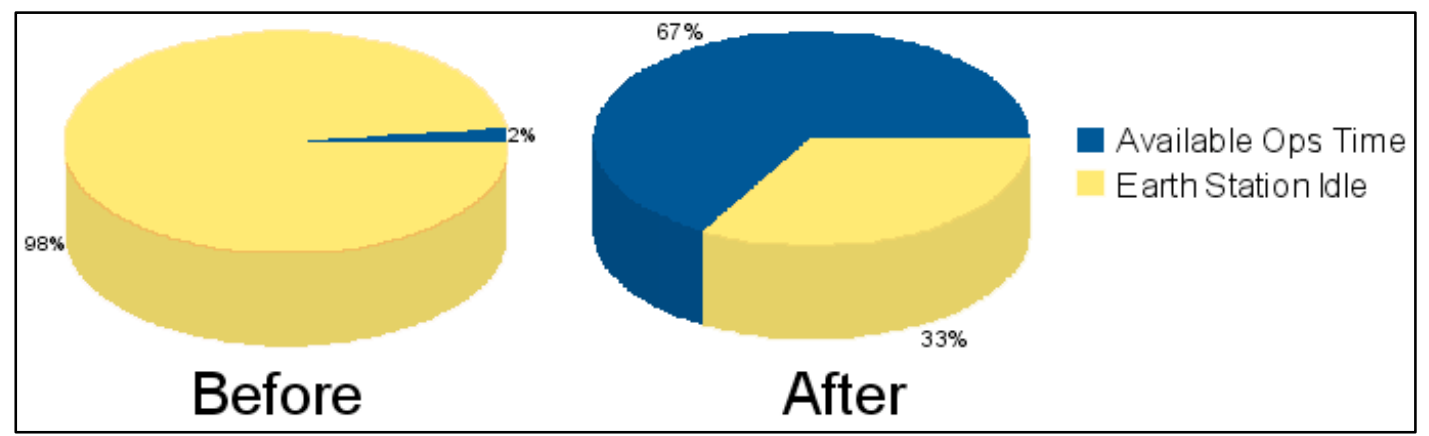

Figure 87. Spacecraft ops availability before and after GENSO ${ }^{26}$ 
The figure below shows the layout of the current Global Educational Network for Satellite Operations (GENSO) member sites and their associated coverage for LEO satellites. The exponential growth of the GENSO project is another enabler to making on-orbit adaptive component utilization a reality, especially for satellites and teams that either do not have an automated ground station or have very limited pass-times and no "steady commanding" built into their satellites onboard operations.

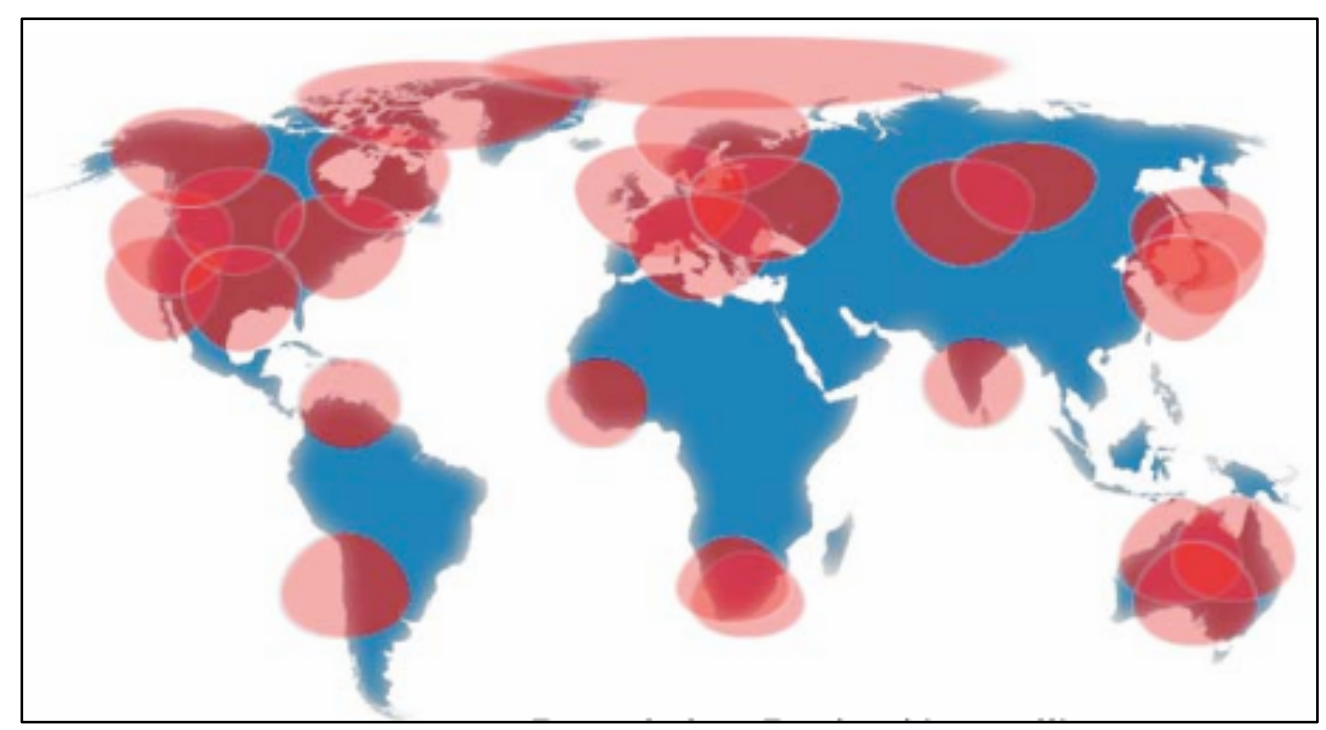

Figure 88. GENSO Network coverage for LEO Satellites ${ }^{27}$

With the help and support of previous Cal Poly PolySat engineers Kyle Leveque (SRI) and Bryan Klofas (SRI) the GENSO effort is taking hold and coming to maturity in the United States. A study regarding how these new capabilities could facilitate this thesis and other innovative concepts is recommended. 


\subsubsection{Passive Thermal Control Improvements}

Based on the knowledge gained regarding the surface properties of the CP satellites there are improvements that could be made to increase the eclipse low temperatures and decrease the sunlit high temperatures. Specifically, utilizing a solder mask overlay with low absorptivity and low emissivity such as a DuPont 4817 or Epson 828 would improve the capability of the satellite to retain what little heat it produces while rejecting the heat incident upon its exposed non-solar panel surfaces. If other options, such as mylar or kapton insulation, were available a single layer of aluminized kapton integrated under the external panels, encapsulating the interior of the satellite would serve a similar purpose and would be a simpler integration.

\subsubsection{Improved Capabilities with Increased Power Budgets}

The power budget available to CubeSats has been increasing rapidly over the past five years. With the advent of a dedicated CubeSat power supply company, ClydeSpace, and the introduction of new $3 \mathrm{U}$ deployable solar panels by Pumpkin and Northrop Grumman, CubeSats are entering the realm of $>50 \mathrm{~W}$ power supplies. This drastic increase in the available power makes CubeSat thermal design both simpler and more challenging. It becomes easier because satellites can now be designed more in-line with industrial satellites. These new power levels allow for cold-biased designs with heaters to protect key subsystems and maintain internal temperatures. The new challenge comes from the fact that these high power CubeSats now have the opposite problem of too 
little thermal energy. They now have to find a way to safely and reliably reject 50 watts of thermal energy. This is a huge amount of energy for a 4 kilogram satellite to reject and if the designers are not careful they could quickly find themselves overheating their satellite's key sensors and electronics.

As an example of the challenges CubeSats face when implementing heater circuits, the COMPASS CubeSat mission launched on 28 April 2008 and had trouble initially contacting their satellite. They had integrated a foil heater similar to the one shown below in Figure 91.

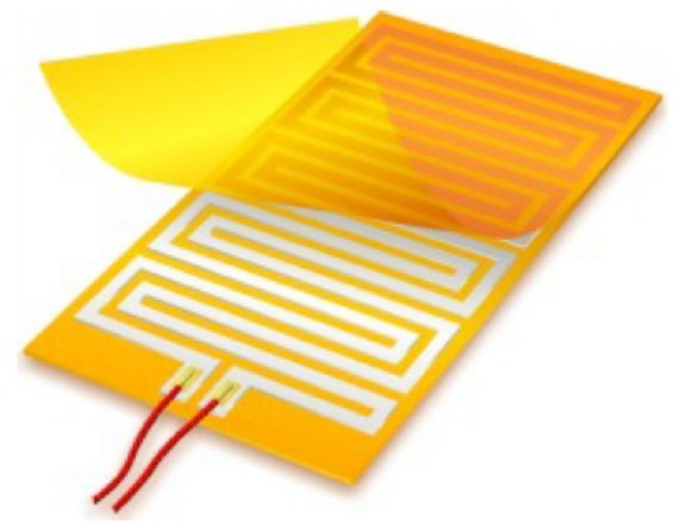

Figure 89. Kapton Enclosed Foil Strip Heater

The COMPASS satellite (University of Aachen, Germany) underwent a reset after in mid-August of 2008 that turned off all the major satellite electronics and then turned them back on. Unfortunately during the off-period the satellite had cooled down enough that when the satellite turned back on it immediately engaged the heater which, in normal fashion for a resistive heater, is a current heavy operation. The current draw forces an under-voltage on the satellite bus, which unfortunately then sends a reset command turning the main bus back off. 
It continued limping along in this fashion until the Aachen team finally, in desperation, released all of their uplink command information to the amateur radio community in the hopes that someone would be able to catch the satellite in between its destructive on-off cycle and alter the thermistor settings that enabled the heater on command. On September $10^{\text {th }} 2008$ the team finally, thanks to amateur satellite operators around the world pinging COMPASS with the adjustment, got it back into a stable configuration with an adjusted temperature setting. This is one example of just how challenging onboard thermal control can be for a CubeSat team and a perfect illustration of how a passive thermal solution is always a safer alternative. 


\section{REFERENCES}

1. Colter, Dauna. "NASA to Attempt Historic Solar Sail Deployment". 26 June 2008. Web. 20 April 2009. Retrieved from <http://www.science.nasa.gov>

2. Gilmore, David G., ed. Spacecraft Thermal Control Handbook; Second Edition. El Segundo, CA: The Aerospace Press, 2002.

3. NASA Office of Safety and Mission Assurance. "NASA Safety Standard: Guidelines and Assessment Procedures for Limiting Orbital Debris NSS 1740.14". Washington, D.C., 1995.

4. Puig Suari, Jordi. "A Low Cost Pico-Satellite Standard for Education and Research," Presentation to Naval Post Graduate School. Monterey, CA. 26, July 2007.

5. Milne, Scott Jr., Daniel Kaufman. "GEVS-SE Rev A General Environmental Verification Specification for STS \& ELV Payloads, Subsystems and Components". Goddard Space Flight Center, Greenbelt, Maryland, 1996.

6. Cal Poly CubeSat Program. CubeSat Design Specification, Rev. 12, 2009.

7. Hoffman, Eric J., Glen H. Fountain. "The System Approach to Successful Space Mission Development”. Johns Hopkins APL Technical Digest, Volume 20, Number 4 (1999)

8. PC/104 Embedded Consortium. (2011) PC/104 - Data sheet, January 2011. [Data file]. Retrieved from http://www.pc104.org/

9. Mortenson, C., "NPS-SCAT; Communications System Design, Test and Integration of NPS's First CubeSat," Master's Thesis, Naval Postgraduate School, 2010.

10. CTi Corporation. "Surface Mount Resistors." [Data file]. Retrieved from <http://ctius.com/>

11. Panasonic Corporation. "ECWU Series SP Cap Polymer Aluminum." [Data file]. Retrieved from <http://Panasonic.com>

12. Dallas Semiconductor. Maxim Division. (2010) 1239M - Data sheet 19-2333 Revision 7, May 2010. [Data file]. Retrieved from <http://datasheets.maxim-ic.com/> 
13. Analog Devices. "AD627AR." [Data file]. Retrieved from <http://octopart.com/ad627aranalog+devices-410862>

14. Judd, Ross Leonard. "Determination of the Emissivity of a Heated Copper Surface". Experiments in Heat Transfer and Thermodynamics. University of Michigan, 1992.

15. Kyte, J.R. A.J. Madden and E.L. Piret, "Natural Convection Heat transfer at Reduced Pressure," Chemical Engineering Progress 49, 12 (1953): 635-662.

16. Spectrolab. "26.8\% Improved Triple Junction (ITJ) Solar Cells." Spectrolab. 4/29/2008. 2/10/2011. <http://www.spectrolab.com/DataSheets/TNJCell/tnj.pdf>.

17. Granger, Robert. Experiments in Heat Transfer and Thermodynamics. New York, NY: The Press Syndicate of the University of Cambridge, 1994

18. Wiebelt, J. A.. Engineering Radiation Heat Transfer. Chicago, IN: Holt Rinehart and Winston Inc., 1965.

19. Larson, Wiley J., James Wertz. Space Mission Analysis and Design. ( $3^{\text {rd }}$ ed.). Torrance, CA. Microcosm and Springer Publishing, 1999.

20. Noe, Christopher. "Design and Implementation of the Communications Subsystem for the Cal Poly CP2 CubeSat Project." Senior Project, California Polytechnic State University, San Luis Obispo, CA, 2005.

21. Infineon Technologies. "Thermal Resistance Theory and Practice SMD Packages"; Edition January 2000. Infineon Technologies AG, Munich, Germany.

22. Benson, Jim, "Thermal Characterization of Packaged Semiconductor Devices." Technical Briefings by Intersil Corporation, December 2002: TB379.3.

23. Naicker, Nathan. "PCB Designer of the 21 st Century Needed." Online Article. EDA Technologies. Retrieved from <http://www.dataweek.co.za/news>.

24. Zhang, K. Xu, and T. R. Jow, J., Solid State Electrochem. Vol 7, 2003.

25. Glassey, Kalia. "Development of an Imager System Optimized for Low-Power, LimitedBandwidth Space Applications." Master's Thesis, California Polytechnic State University, San Luis Obispo, CA, 2009. 
26. Anderson, Jason. "Autonomous Satellite Operations for CubeSat Satellites." Master's Thesis, California Polytechnic State University, San Luis Obispo, CA, 2010.

27. Melville, Neil. "Global Educational Network for Satellite Operations." International Space Education Board, Hyderabad, September 2007.

28. Panasonic Corp. "CGA103450A Cobalt Series." February 2010. Web. 3 March 2011. <http://www.datasheetcatalog.com/datasheets_pdf/C/G/A/1/CGA103450.shtml>.

29. ISOLA Corp. "FR406 Data Sheet." 16 March 2007. Web. 4 May 2007. Retrieved from $<\mathrm{http}: / /$ www.isola-group.com/docs>.

30. Kodak Corp. "KAC-9638 IMAGER PRODUCT SUMMARY." August 2006. Web. 29 May 2007. <http://www.kodak.com/global/plugins/acrobat/en/business/ISS/datasheet>.

31. Hamilton, D.C. Morgan, W.R.. NACA Tech. Note TN-2836, 1952.

32. Nagai, Takayuki. "Surface Mount Component Failure Thermal Cycling." Espec Test Center, Espec Technology Report No. 14. September 15, 2007.

33. Citizen Corporation. "HCM49 At-Cut Crystal; Data Sheet". Web. Retrieved from http://citizencrystal.com.

34. Dallas Semiconductor. Maxim Division. (2010) DS3231 - Data sheet 19-5170 Revision 8, June 2010. [Data file]. Retrieved from http://datasheets.maxim-ic.com/

35. Linden, David; Reddy, Thomas B. Handbook of Batteries -Third Edition. New York, NY: McGraw-Hill, 2002

36. Kwona, Ou Jung. Wanga, Chao-Yang. "Lithium Deposition in the Anode of Automotive Liion Batteries", Pennsylvania State University, 2009.

37. Day, Chris. "The Design of an Efficient, Elegant, and Cubic Pico-Satellite Electronics System." Master's Thesis, California Polytechnic State University, 2004.

38. Rupprecht, Mike. "GeneSat-1 Telemetry Decoder." Web. 21 August 2010. Retreived from $<$ http://www.dk3wn.info/software.shtml> 


\section{APPENDIX A. CP3 Power Storage Thermal \& Life Properties ${ }^{28}$}

Shown below in Figure 90 are the properties of the batteries chosen for CP3.

Note typical discharge by temperature and typical life characteristics.

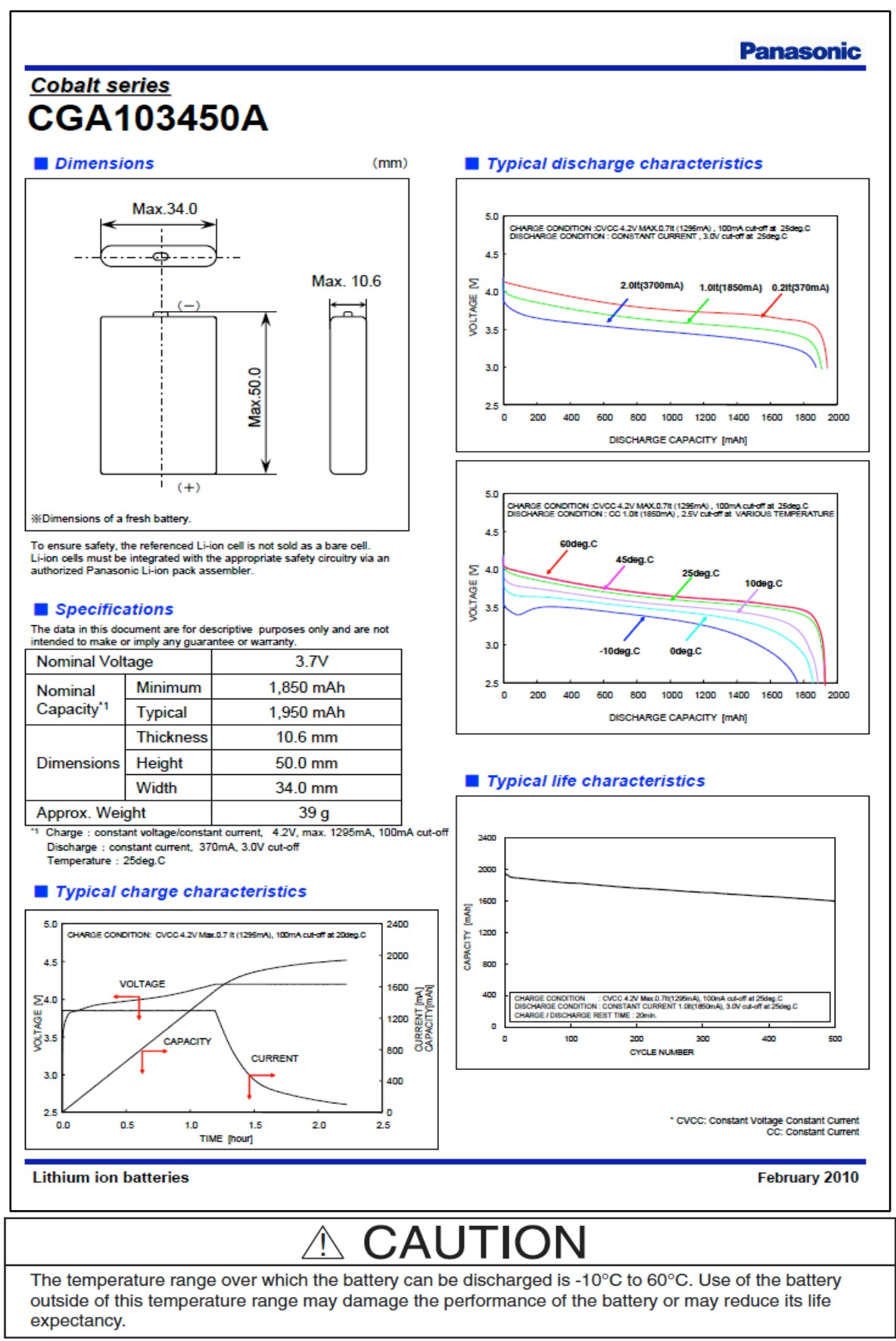

Figure 90. Panasonic Prismatic Lithium Ion Operating Characteristics 
APPENDIX B. ISOLA FR406 PCB Material Properties Sheet ${ }^{29}$

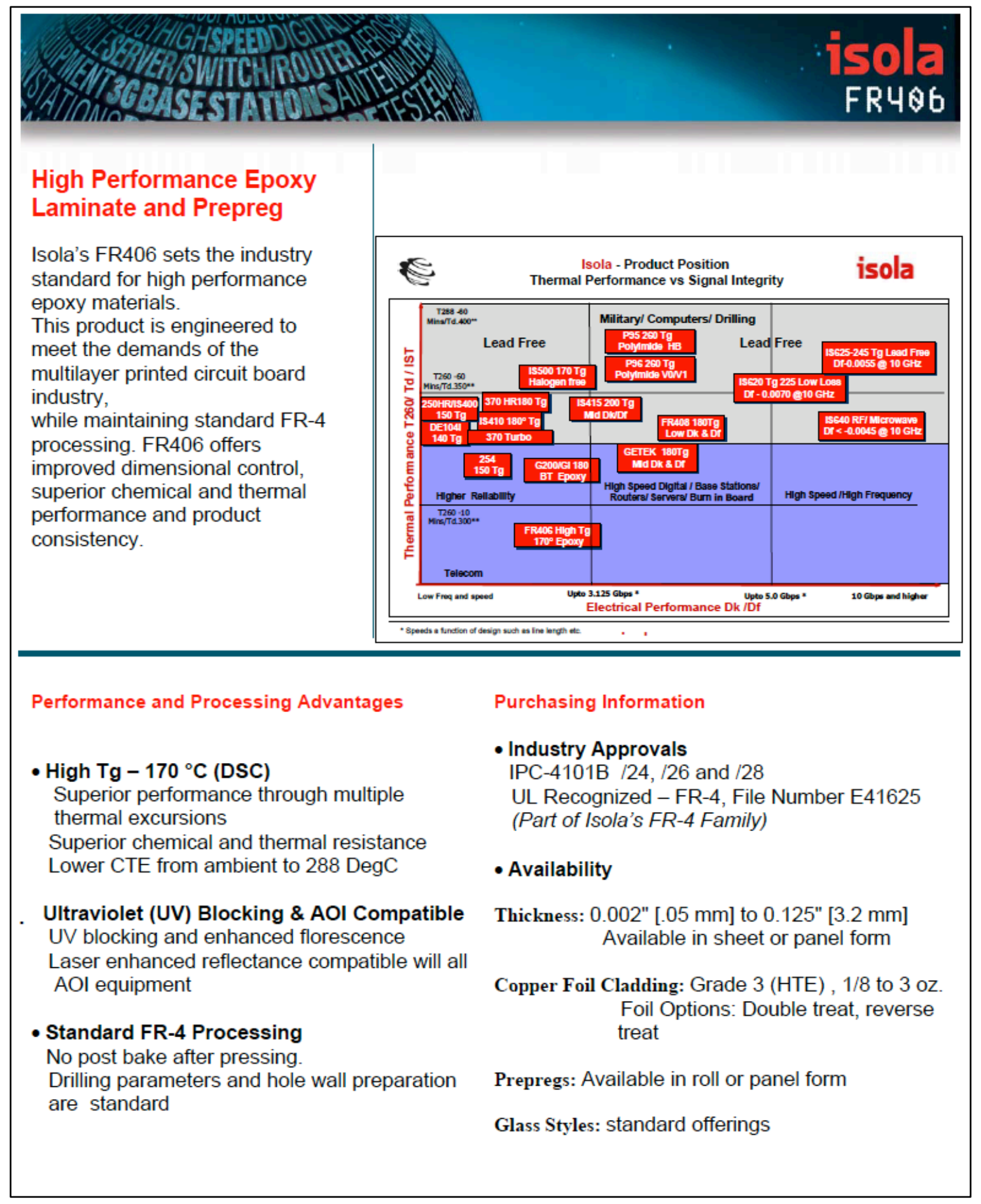

Figure 91. FR406 Material Properties Sheet 


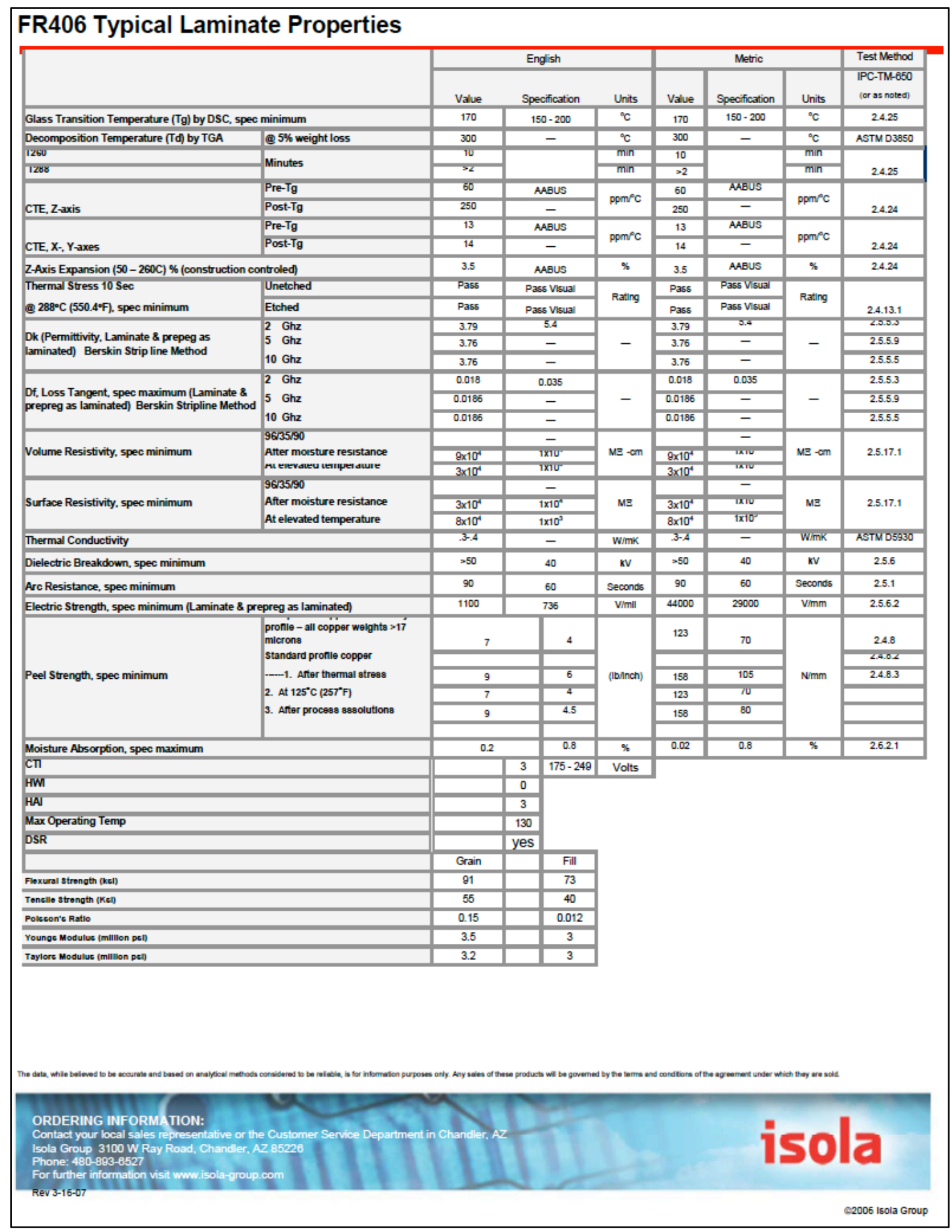

Figure 92. FR406 Material Properties Sheet (cont'd) 
Appendix C CP3 Payload (KAC-9638/48) Thermal Properties ${ }^{30}$

The properties for the CP3 payload camera KAC-9638 are shown below. Note

that the monochromatic CMOS (below figure 93 ) has only $67 \%$ of the thermal

operating temperature range of the Color CMOS shown in the second figure.

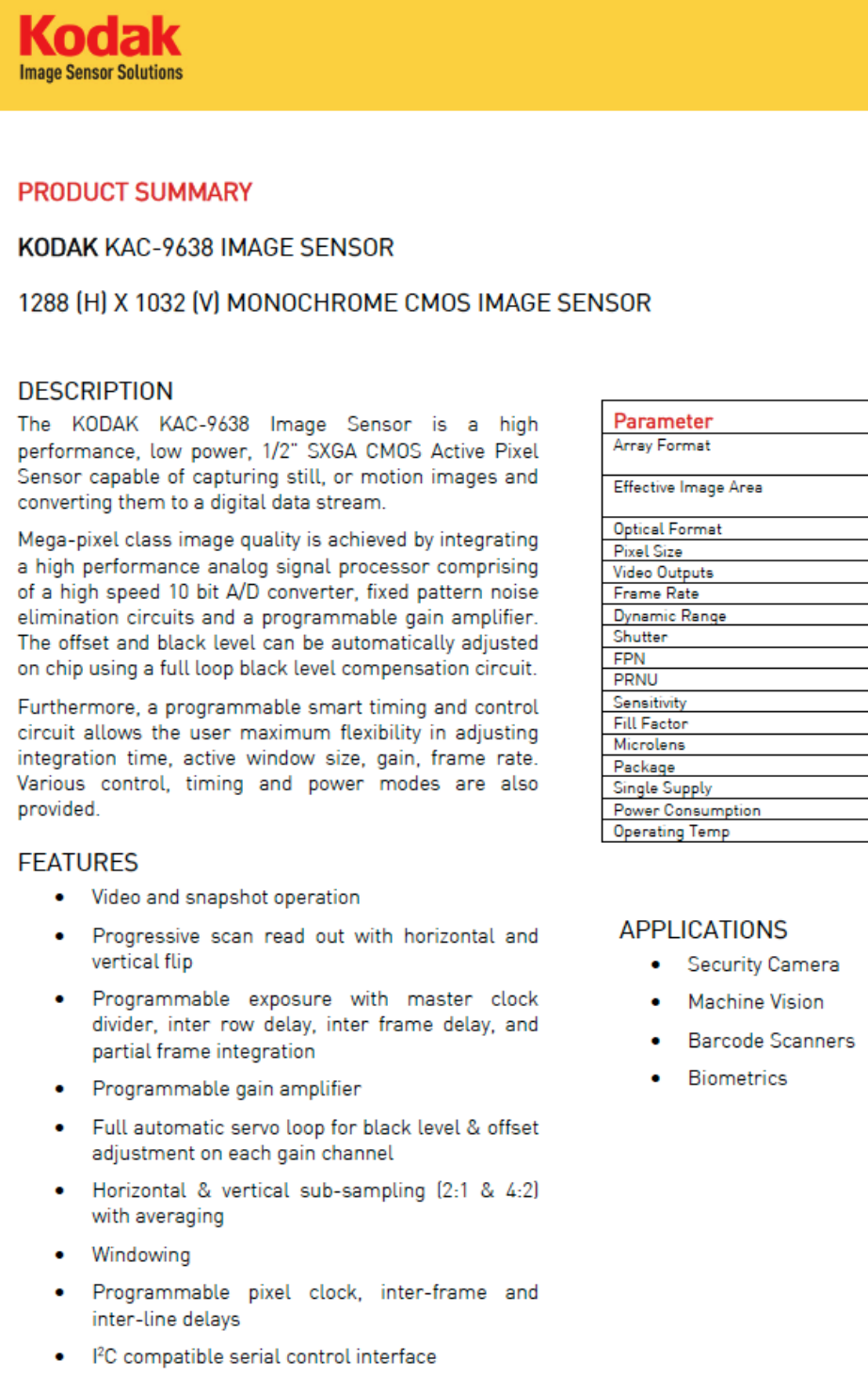

Mega-pixel class image quality is achieved by integrating a high performance analog signal processor comprising of a high speed 10 bit $A / D$ converter, fixed pattern noise elimination circuits and a programmable gain amplifier. The offset and black level can be automatically adjusted on chip using a full loop black level compensation circuit.

Furthermore, a programmable smart timing and control circuit allows the user maximum flexibility in adjusting integration time, active window size, gain, frame rate. Various control, timing and power modes are also provided.

\section{FEATURES}

- Video and snapshot operation

- Progressive scan read out with horizontal and vertical flip

- Programmable exposure with master clock divider, inter row delay, inter frame delay, and partial frame integration

- Programmable gain amplifier

- Full automatic servo loop for black level \& offset adjustment on each gain channel

- Horizontal \& vertical sub-sampling [2:1 \& 4:2] with averaging

- Windowing

- Programmable pixel clock, inter-frame and inter-line delays

- $\quad \mathrm{I}^{2} \mathrm{C}$ compatible serial control interface

$$
\begin{aligned}
& \text { APPLICATIONS } \\
& \text { - Security Camera } \\
& \text { - Machine Vision } \\
& \text { - Barcode Scanners } \\
& \text { - Biometrics }
\end{aligned}
$$

\begin{tabular}{|l|l|}
\hline Parameter & Typical Value \\
\hline Array Format & $\begin{array}{l}\text { Total: } 1032 \times 1312 \\
\text { Active: } 1032(\mathrm{~V} \times 1288[\mathrm{H}]\end{array}$ \\
\hline Effective Image Area & $\begin{array}{l}\text { Total: } 6.192 \mathrm{~mm} \times 7.872 \mathrm{~mm} \\
\text { Active: } 6.192 \mathrm{~mm} \times 7.728 \mathrm{~mm}\end{array}$ \\
\hline Optical Format & $1 / 2^{*}$ \\
\hline Pixel Size & $6.0 \mu \mathrm{m} \times 6.0 \mu \mathrm{m}$ \\
\hline Video Outputs & $8 \& 10$ Bit Digital \\
\hline Frame Rate & 18 frames per second \\
\hline Dynamic Range & $55 \mathrm{~dB}$ \\
\hline Shutter & Rolling reset \\
\hline FPN & $0.2 \%$ \\
\hline PRNU & $1.7 \%$ \\
\hline Sensitivity & $2.40 \mathrm{~V} / \mathrm{lux} \times^{*} \mathrm{~s}$ \\
\hline Fill Factor & $49 \%$ \\
\hline Microlens & none \\
\hline Package & $48 \mathrm{LCC}$ \\
\hline Single Supply & $3.0 \mathrm{~V} \pm 10 \%$ \\
\hline Power Consumption & $150 \mathrm{~mW}$ \\
\hline Operating Temp & $10^{\circ} \mathrm{C}$ to $50^{\circ} \mathrm{C}$ \\
\hline
\end{tabular}

Figure 93. KAC-9638 Monochromatic CMOS Operating Temperature Limits 
The properties for the CP3 payload camera KAC-9648 are shown below in figure

94. Note that it has a $33 \%$ wider thermal operating temperature tolerance than the monochromatic CMOS.

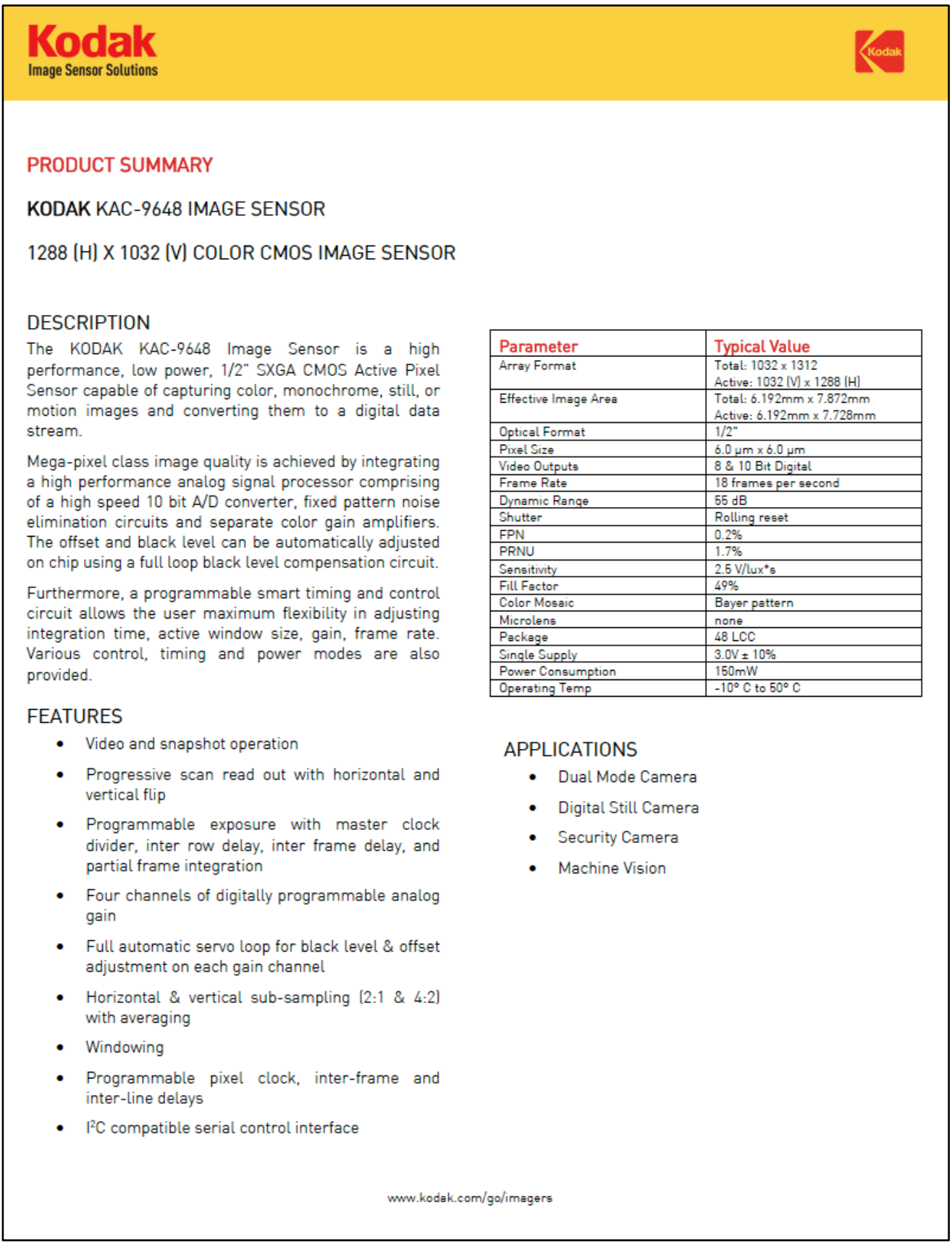

Figure 94. KAC-9648 Color CMOS Operating Temperature Limits 


\section{Appendix D - Populated PCB Calculations \& Adjustments}

The tables below were used to calculate the adjusted surface properties based on component sizes, component surface properties and populated numbers. The first step was to quantify the representative surface mount components, count and number them and finally calculate the adjusted surface emissivity and absorptivity based on the replaced and added surface area amounts. Table 24 below shows the initial calculations for component size, replaced surface area amount, added surface area amount and the averaged properties for both.

Table 24. Surface Area by Representative Component \& Property Adjustment

\begin{tabular}{|c|c|c|c|c|c|c|}
\hline \multicolumn{7}{|c|}{ Populated PCB Calculations } \\
\hline \multicolumn{7}{|c|}{ Surface Mount Capacitors } \\
\hline$\underline{L}$ WW & & $\underline{\mathrm{H}^{*} \mathrm{~W}}$ & \multicolumn{2}{|r|}{ Leads } & Extra Surface Area & $\underline{\text { Replaced Surface Area }}$ \\
\hline 0.5 & 0.5 & & 0.25 & 0.125 & 1.50 & 0.75 \\
\hline Surface Emissivity & & & & & Avg Emissivity (Extra) & Avg Emissivity (Replaced) \\
\hline 0.9 & 0.9 & & 0.02 & 0.02 & 0.61 & 0.61 \\
\hline \multicolumn{7}{|c|}{ Surface Mount Resistors } \\
\hline$\underline{\mathrm{L} * \mathrm{~W}} \quad \underline{\mathrm{L}} \quad \underline{\mathrm{H}}$ & & $\underline{\mathrm{H}^{*} \mathrm{~W}}$ & & Leads & Extra Surface Area & $\underline{\text { Replaced Surface Area }}$ \\
\hline 0.5 & 0.35 & & 0.175 & 0.125 & 1.05 & 0.75 \\
\hline Surface Emissivity & & & & & Avg Emissivity (Extra) & Avg Emissivity (Replaced) \\
\hline 0.9 & 0.81 & & 0.02 & 0.02 & 0.55 & 0.61 \\
\hline \multicolumn{7}{|l|}{ Maxim 1239} \\
\hline$\underline{L} * \mathrm{~W}$ & & $\underline{\mathrm{H}^{*} \mathrm{~W}}$ & & Leads & Extra Surface Area & Replaced Surface Area \\
\hline 19.071 & 7.5795 & & 6.045 & 4.24 & 27.25 & 27.55 \\
\hline Surface Emissivity & & & & & Avg Emissivity (Extra) & Avg Emissivity (Replaced) \\
\hline 0.9 & 0.9 & & 0.9 & 0.02 & 0.90 & 0.63 \\
\hline \multicolumn{7}{|l|}{ AD627AR } \\
\hline$\underline{L * W}$ & & $\underline{\mathrm{H}^{*} \mathrm{~W}}$ & & Leads & Extra Surface Area & Replaced Surface Area \\
\hline 19.11 & 7.595 & & 6.045 & 3.44 & 27.28 & 25.99 \\
\hline Surface Emissivity & & & & & Avg Emissivity (Extra) & Avg Emissivity (Replaced) \\
\hline 0.9 & 0.9 & & 0.9 & 0.02 & 0.90 & 0.67 \\
\hline
\end{tabular}

Using the representative components each board in the CP system was quantified in terms of total surface area (for the CP3 configuration), component population and then calculated to find the actual emissive and absorptive 
properties of each board, top and bottom. Tables 25-30 contain the results for each of these calculations along with a summary in Table 31 of the total system area (external) and its averaged property, taking into account the anodized aluminum side rails. These are the values utilized in all supporting calculations and simulations (post Appendix 5 Initial Calculation).

Table 25. Side Panel Interior Emissivity Calculation (Correction)

\begin{tabular}{|c|c|c|c|c|}
\hline $\begin{array}{l}\text { Solar Panels } \\
\text { Interior }\end{array}$ & "Resistors" & "Capacitors" & "Maxims" & "ADCs" \\
\hline \multirow[b]{2}{*}{ Extra Surface Area } & 48.00 & 35.00 & 4.00 & 3.00 \\
\hline & 50.40 & 52.50 & 109.00 & 81.84 \\
\hline Replaced Surface Area & 36.00 & 26.25 & 110.20 & 82.65 \\
\hline Avg. Emissivity (Extra) & 0.55 & 0.61 & 0.90 & 0.90 \\
\hline Avg. Emissivity (Replaced) & 0.61 & 0.61 & 0.63 & 0.67 \\
\hline Weighted E By Area (Extra) & 27.55 & 31.85 & 98.10 & 73.66 \\
\hline \multirow[t]{7}{*}{ Weighted E By Area (Repl) } & 21.84 & 15.93 & 69.33 & 55.13 \\
\hline & TOTAL $\left(\mathrm{mm}^{\wedge} 2\right)$ & TOTAL $\left(\mathrm{m}^{\wedge} 2\right)$ & \% of Panel & $\%$ of Panel \\
\hline & 293.74 & 0.00029 & $4 \%$ & $7 \%$ \\
\hline & 255.11 & 0.00026 & $3 \%$ & \\
\hline & E AVG (SA) & E AVG (EX + RE) & New $\mathrm{E}$ & Previous E \\
\hline & 0.79 & 0.72 & 0.81 & 0.90 \\
\hline & 0.64 & & & $10 \%$ \\
\hline
\end{tabular}

Table 26. Side Panel Exterior Absorptivity and Emissivity Calculation (Correction)

\begin{tabular}{|lcccr|r|}
\hline $\begin{array}{l}\text { Solar Panels } \\
\text { Exterior }\end{array}$ & Cells & \multicolumn{3}{c|}{ Leads } \\
\hline & 2 & 6.00 & & \\
Surface Area & 54.6 & 2.32 & Averaged & \% Change \\
Avg. Emissivity & 0.85 & 0.02 & 0.82 & $4.0 \%$ \\
Avg. Absorptivity & 0.92 & 0.3 & 0.89 & $2.7 \%$ \\
Averaged E For Panel & Remainder of Panel is Soldermask @.9 & & 0.84 \\
\hline
\end{tabular}

Table 27. C\&DH Board Emissivity Calculation (Correction) 


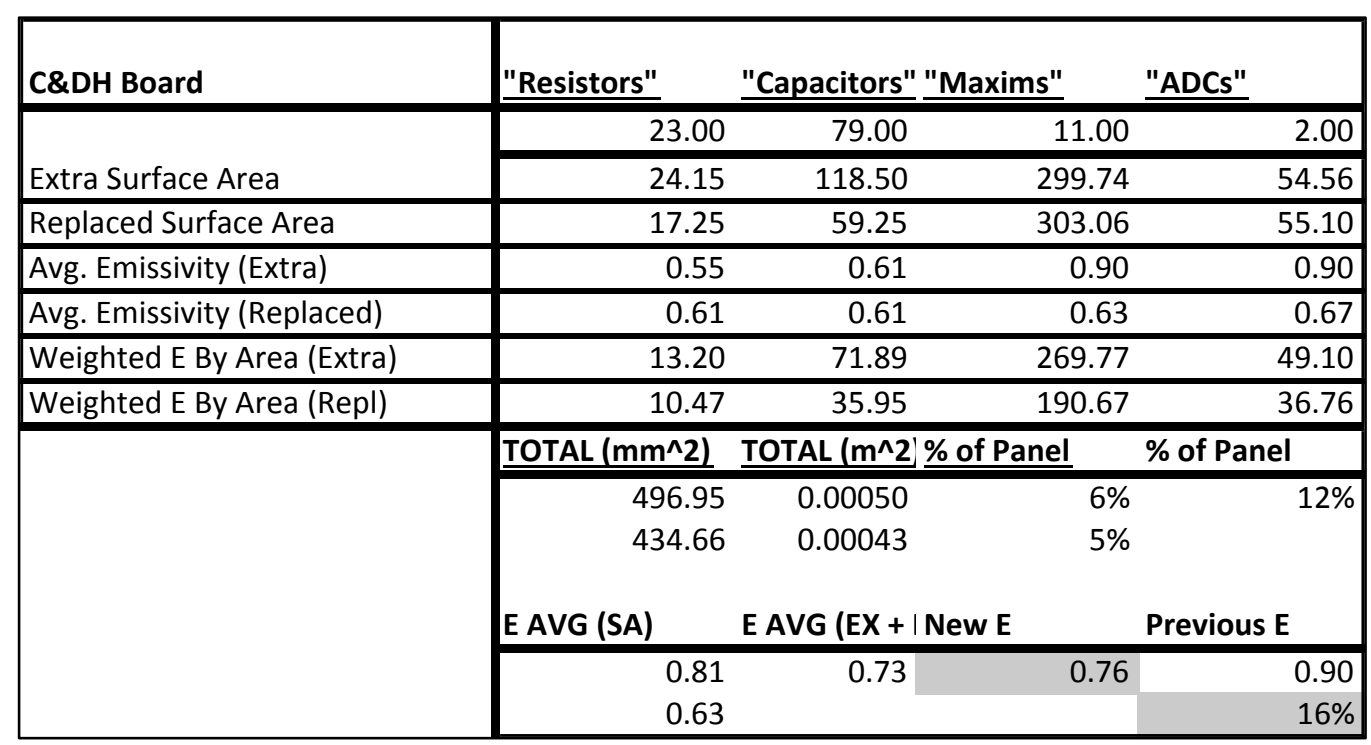

Table 28. Standard Side Panel Corrected Emissivity and Absorptivity Values

\begin{tabular}{|lr|}
\hline Standard Side-Panels (1-3) \\
PCB Panel Emissivity & 0.84 \\
PCB Panel Absorptivity & 0.89 \\
PCB Panel Area (m^2) & 0.0080487 \\
Primary Structure Emissivity & 0.86 \\
Primary Structure Absorptivity & 0.73 \\
Primary Structure Area (m^^2) & 0.00150876 \\
\hline PANEL (Emissivity) & $\mathbf{0 . 8 4}$ \\
PANEL (Absorptivity) & $\mathbf{0 . 8 6}$ \\
\hline
\end{tabular}

Table 29. +Z \& -Z Side Panel Corrected Emissivity and Absorptivity Values 


\begin{tabular}{|lr|}
\hline$+Z$ \& $-Z$ Side-Panels' & \\
PCB Panel Emissivity & 0.84 \\
PCB Panel Absorptivity & 0.89 \\
PCB Panel Area (m^2) & 0.0080487 \\
Primary Structure Emissivity & 0.86 \\
Primary Structure Absorptivity & 0.73 \\
Primary Structure Area (m^^2) & 0.00100584 \\
& \\
\hline PANEL (Emissivity) & $\mathbf{0 . 8 4}$ \\
PANEL (Absorptivity) & $\mathbf{0 . 8 7}$ \\
\hline
\end{tabular}

Table 30. Antenna Side Panel Corrected Emissivity and Absorptivity Values

\begin{tabular}{|lr|}
\hline Antenna Side-Panel & \\
PCB Panel Emissivity & 0.84 \\
PCB Panel Absorptivity & 0.89 \\
PCB Panel Area (m^^2) & 0.0066717 \\
Primary Structure Emissivity & 0.86 \\
Primary Structure Absorptivity & 0.73 \\
Primary Structure Area (m^^2) & 0.00150876 \\
Delrin (black) Emissivity & 0.87 \\
Delrin (black) Absorptivity & 0.96 \\
Delrin Area & 0.001377 \\
\hline PANEL (Emissivity) & $\mathbf{0 . 8 5}$ \\
PANEL (Absorptivity) & $\mathbf{0 . 8 7}$ \\
\hline
\end{tabular}

Table 31. Corrected Satellite Surface Averages (Satellite Whole)

\begin{tabular}{|lr|}
\hline SATELLITE SURFACE AVERAGED \\
Emissivity & 0.84 \\
Absorptivity & 0.87 \\
E/Alpha & 0.97 \\
\hline
\end{tabular}




\section{APPENDIX E. Sierra Proto Express Solder Mask Properties}

William,

I could not find a lot of information regarding the thermal properties of LPI solder mask. IVe attached the data sheet for the solder mask that we use but I saw no mention of thermal conductivity, emissivity or absorptivity. The link below does have a callout, but its not specific on the type of mask that is being referenced.

http://203.66.215.134/webprj/rd/itdas_intro2.htm

LeBOY

LeBoy Hendy

Technical Specialist

Sierra Proto Express

408-735-7137 ext.107

- Hide quoted text -

-----Original Message----

From: wdwhalen@gmail.com [mailto:wdwhalen@gmail.com] On Behalf Of William Whalen

Sent: Thursday, June 28, 2007 11:49 PM

To: LeBoy Hendy

Subject: Re: Data sheet for FR406

Dear Leroy,

On our PCB boards that we've purchased from Proto express, the top layer is typically coated in either black or blue enamel solder mask. Do you have any information regarding the thermal properties on this? We're trying to figure out the radiative properties specifically, but any material data you can obtain would be helpful.

Thanks,

William

Figure 95. Sierra Proto Express Tech Specialist Reference Letter 
APPENDIX F. INITIAL CALCULATIONS (HISTORICAL REFERENCE)

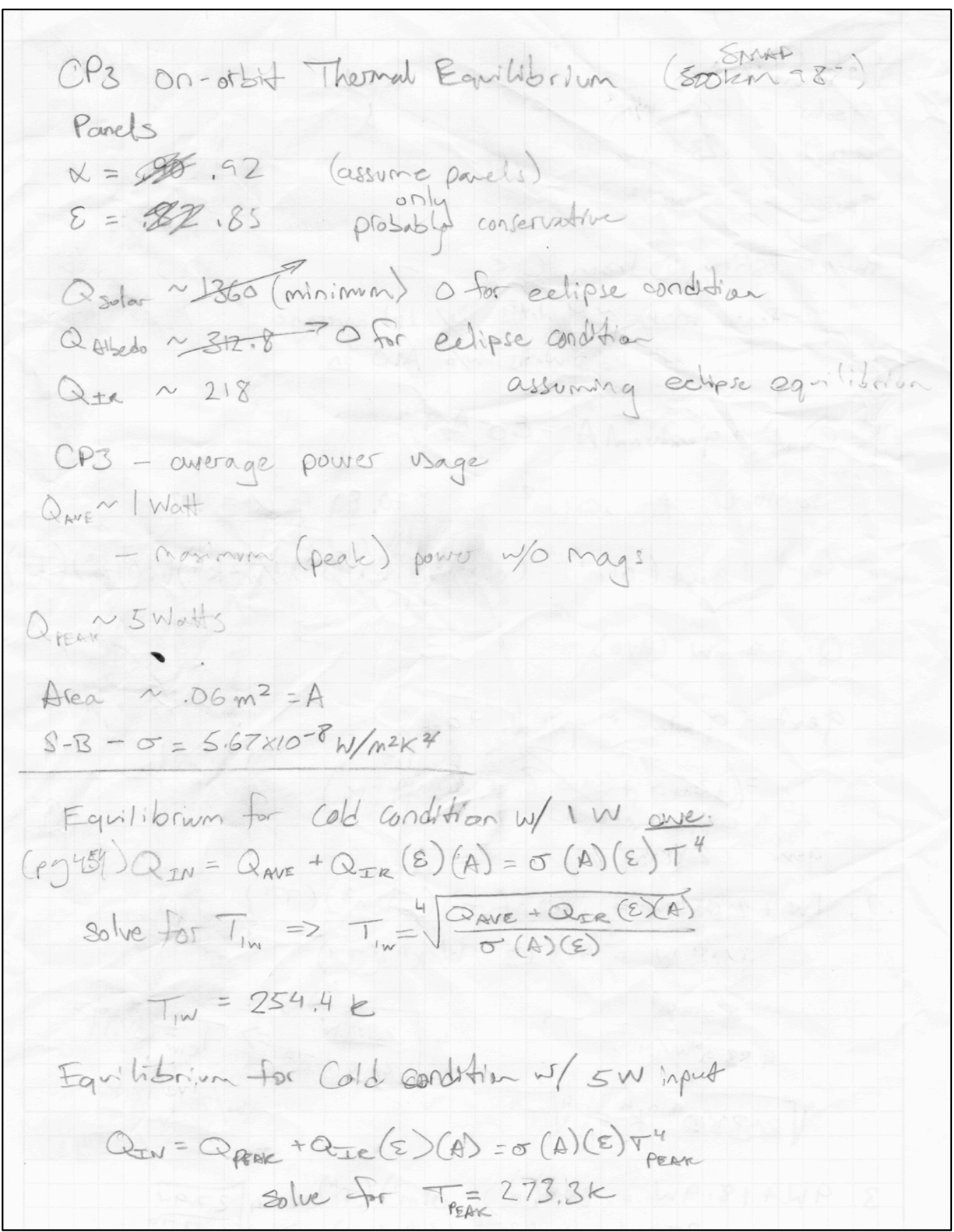

Figure 96. Initial Proof-of-Concept Calculation with Assumptions 
APPENDIX G. Projected Area Calculations

Maximum projected area calculation table. Cont'd on following page.

Table 32. Tumbling Satellite Panel Area Calculations

\begin{tabular}{|c|c|c|c|c|c|c|}
\hline \multicolumn{7}{|l|}{ CPX Area Projection Calculator } \\
\hline Solar Flux & \multicolumn{2}{|r|}{ Face Area } & \multicolumn{2}{|c|}{$100 \mathrm{~mm} \times 100 \mathrm{~mm}$} & \multicolumn{2}{|c|}{$10000 \mathrm{~mm} \wedge 2$} \\
\hline$\underline{X \text { Face Area }}$ & Alpha (degrees) & Beta (degrees) & XFace & YFace & $\frac{2 \text { Face }}{1000}$ & $\begin{aligned} \text { Total Area } \\
1000 \\
\end{aligned}$ \\
\hline$A x=A^{*} \sin (\text { alpha })^{*} \cos ($ beta $)$ & 5 & 0 & 87.15574 & 0 & 996.1947 & 1083.35 \\
\hline Y Face Area & $\begin{array}{l}10 \\
15\end{array}$ & 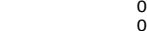 & $\begin{array}{r}173.6482 \\
258.819\end{array}$ & $\begin{array}{c}0 \\
0\end{array}$ & $\begin{array}{l}984.8078 \\
965.958\end{array}$ & $\begin{array}{l}1158.456 \\
1224.745\end{array}$ \\
\hline & 20 & 0 & 342.0201 & 0 & 939.6926 & 1281.713 \\
\hline$A y=A^{*} \sin (b e t a)$ & $\begin{array}{l}25 \\
30\end{array}$ & $\begin{array}{l}0 \\
0\end{array}$ & $\begin{array}{r}422.6183 \\
500\end{array}$ & $\begin{array}{l}0 \\
0 \\
0\end{array}$ & $\begin{array}{l}906.3078 \\
866.0254\end{array}$ & $\begin{array}{l}1328.926 \\
1366.025\end{array}$ \\
\hline$\underline{Z \text { Face Area }}$ & 35 & 0 & 573.5764 & 0 & 819.152 & 1392.728 \\
\hline$A z=A^{*} \cos (\text { alpha })^{*} \cos ($ beta $)$ & $\begin{array}{l}40 \\
45\end{array}$ & $\begin{array}{c}0 \\
0 \\
0\end{array}$ & $\begin{array}{l}642.7876 \\
707.1068\end{array}$ & ${ }_{0}^{0}$ & $\begin{array}{l}766.0444 \\
7071068\end{array}$ & $\begin{array}{l}1408.832 \\
1414.214\end{array}$ \\
\hline & 50 & 0 & 766.0444 & 0 & 642.7876 & $\begin{array}{l}1408.832 \\
14082728\end{array}$ \\
\hline Total Area & 60 & 0 & $\begin{array}{r}819.152 \\
866.0254\end{array}$ & $\begin{array}{l}0 \\
0\end{array}$ & $\begin{array}{r}573.5764 \\
500\end{array}$ & $\begin{array}{l}1392.728 \\
1366.025\end{array}$ \\
\hline Atot $=A x+A y+A z$ & $\begin{array}{l}65 \\
70\end{array}$ & $\begin{array}{l}0 \\
0 \\
0\end{array}$ & $\begin{array}{l}906.3078 \\
939.6926\end{array}$ & $\begin{array}{l}0 \\
0 \\
0\end{array}$ & $\begin{array}{l}422.6183 \\
342.0201\end{array}$ & $\begin{array}{l}1328.926 \\
1281.713\end{array}$ \\
\hline & 75 & 0 & 965.9258 & 0 & 258.819 & 1224.745 \\
\hline MAXIMUM PROJECTED AREA & $\begin{array}{l}80 \\
85\end{array}$ & $\begin{array}{l}0 \\
0\end{array}$ & $\begin{array}{l}984.8008 \\
996.1947\end{array}$ & & $\begin{array}{l}173.6482 \\
87.15574\end{array}$ & $\begin{array}{r}1158.456 \\
1083.35\end{array}$ \\
\hline & 90 & 0 & 1000 & 0 & $6.13 E-14$ & $\begin{array}{r}1000 \\
108335\end{array}$ \\
\hline$\frac{1732.323667}{173.2032367 \mathrm{~cm}^{\wedge} 2}$ & $\begin{array}{l}0 \\
5\end{array}$ & $\begin{array}{r}5 \\
5 \\
\end{array}$ & 86.82409 & $\begin{array}{l}87.15574 \\
87.15574\end{array}$ & $\begin{array}{l}996.1947 \\
992.4039\end{array}$ & $\begin{array}{r}11883.35 \\
1166.384\end{array}$ \\
\hline $0.017320324 \mathrm{~m}^{\wedge} 2$ & $\begin{array}{l}10 \\
15\end{array}$ & $\begin{array}{c}5 \\
5\end{array}$ & $\begin{array}{l}172.9874 \\
257.8342\end{array}$ & $\begin{array}{l}87.15574 \\
87.15574\end{array}$ & $\begin{array}{l}998.0603 \\
962.2502 \\
\end{array}$ & $\begin{array}{r}124.203 \\
1307.24\end{array}$ \\
\hline & 20 & 5 & 340.7187 & 87.15574 & 936.1168 & 1363.991 \\
\hline & $\begin{array}{l}25 \\
30\end{array}$ & $\begin{array}{r}5 \\
5\end{array}$ & $\begin{array}{l}421.0101 \\
498.0973\end{array}$ & $\begin{array}{l}87.15554 \\
87.1574\end{array}$ & $\begin{array}{r}902.859 \\
862.7299\end{array}$ & $\begin{array}{l}1411.025 \\
1447.983\end{array}$ \\
\hline & 35 & 5 & 571.3938 & 87.15574 & 816.0349 & $\begin{array}{l}1474.585 \\
\end{array}$ \\
\hline & 40 & 5 & 640.3416 & 87.15574 & 763.1294 & 1490.627 \\
\hline & $\begin{array}{l}45 \\
50\end{array}$ & $\begin{array}{l}5 \\
5\end{array}$ & $\begin{array}{r}704.446 \\
763.1294\end{array}$ & $\begin{array}{l}87.15554 \\
87.15574\end{array}$ & $\begin{array}{r}704.416 \\
640.3416\end{array}$ & $\begin{array}{l}1495.9888 \\
1490.627\end{array}$ \\
\hline & $\begin{array}{l}55 \\
60\end{array}$ & $\begin{array}{l}5 \\
5 \\
5\end{array}$ & $\begin{array}{l}816.0349 \\
86279299\end{array}$ & $\begin{array}{l}87.15574 \\
87.15754\end{array}$ & $\begin{array}{r}571.3938 \\
4490973\end{array}$ & $\begin{array}{l}1474.584 \\
1479983\end{array}$ \\
\hline & 65 & 5 & $\begin{array}{r}902.859 \\
936.114\end{array}$ & 87.15574 & 421.0101 & $\begin{array}{l}1411.025 \\
\end{array}$ \\
\hline & $\begin{array}{l}70 \\
75\end{array}$ & $\begin{array}{l}5 \\
5 \\
5\end{array}$ & $\begin{array}{l}936.1168 \\
9622502\end{array}$ & $\begin{array}{l}87.15574 \\
87\end{array}$ & $\begin{array}{l}340.7187 \\
5787\end{array}$ & $\begin{array}{r}1363.991 \\
1307.24\end{array}$ \\
\hline & 80 & 5 & 981.0603 & $\begin{array}{l}87.15574 \\
\end{array}$ & $\begin{array}{l}172.9874 \\
172.98\end{array}$ & $\begin{array}{l}1241.203 \\
\end{array}$ \\
\hline & $\begin{array}{l}85 \\
90\end{array}$ & $\begin{array}{l}5 \\
5 \\
\end{array}$ & $\begin{array}{l}992.4039 \\
996.1947\end{array}$ & $\begin{array}{l}87.15574 \\
87.15574\end{array}$ & $\begin{array}{r}86.82409 \\
6\end{array}$ & $\begin{array}{r}1166.384 \\
1083.35\end{array}$ \\
\hline & $\begin{array}{r}90 \\
0\end{array}$ & 10 & $\begin{array}{r}996.1947 \\
0\end{array}$ & $\begin{array}{l}87.15574 \\
173.6482\end{array}$ & $\begin{array}{r}6.11-14 \\
984.8078\end{array}$ & $\begin{array}{r}11883.35 \\
1158.456\end{array}$ \\
\hline & 5 & 10 & $\begin{array}{r}85.83165 \\
17101\end{array}$ & $\begin{array}{l}173.6482 \\
1736482\end{array}$ & 981.0603 & $\begin{array}{r}1240.54 \\
\end{array}$ \\
\hline & $\begin{array}{l}10 \\
15\end{array}$ & 10 & $\begin{array}{r}171.0101 \\
25488\end{array}$ & $\begin{array}{l}173.6482 \\
173648\end{array}$ & $\begin{array}{l}969.8463 \\
\quad 951251\end{array}$ & $\begin{array}{l}1314.505 \\
1379786\end{array}$ \\
\hline & $\begin{array}{l}15 \\
20\end{array}$ & 10 & $\begin{array}{r}234.8881 \\
336.8241\end{array}$ & $\begin{array}{l}173.6482 \\
\end{array}$ & $\begin{array}{c}951.25252 \\
925.4166\end{array}$ & $\begin{array}{l}1435.889 \\
\end{array}$ \\
\hline & 25 & 10 & $\begin{array}{l}416.1977 \\
\end{array}$ & $\begin{array}{l}173.6482 \\
173.645\end{array}$ & 892.5389 & $\begin{array}{l}1482.385 \\
\end{array}$ \\
\hline & $\begin{array}{l}30 \\
35\end{array}$ & $\begin{array}{l}10 \\
10\end{array}$ & $\begin{array}{l}492.4039 \\
564.8625\end{array}$ & $\begin{array}{l}173.6482 \\
173.648\end{array}$ & $\begin{array}{l}852.8685 \\
8506.707\end{array}$ & $\begin{array}{l}1518.921 \\
1545.218\end{array}$ \\
\hline & 40 & 10 & 633.0222 & $\begin{array}{l}173.6482 \\
\end{array}$ & $\begin{array}{l}754.4065 \\
7\end{array}$ & $\begin{array}{l}1561.077 \\
\end{array}$ \\
\hline & $\begin{array}{l}45 \\
50\end{array}$ & $\begin{array}{l}10 \\
10\end{array}$ & $\begin{array}{l}696.3642 \\
754.4065\end{array}$ & $\begin{array}{l}173.6482 \\
173.6482\end{array}$ & $\begin{array}{l}696.3642 \\
633.0222\end{array}$ & $\begin{array}{l}1566.3777 \\
1561.077\end{array}$ \\
\hline & 55 & 10 & $\begin{array}{l}154.405 \\
806.7073\end{array}$ & $\begin{array}{l}173.6482 \\
17382\end{array}$ & 564.8625 & 1545.218 \\
\hline & 60 & 10 & $\begin{array}{l}852.8685 \\
\end{array}$ & 173.6482 & $\begin{array}{l}492.4039 \\
\quad\end{array}$ & 1518.921 \\
\hline & $\begin{array}{l}65 \\
70\end{array}$ & $\begin{array}{l}10 \\
10\end{array}$ & 892.5389 & $\begin{array}{l}173.6482 \\
173648\end{array}$ & 46.1977 & 1482.385 \\
\hline & $\begin{array}{l}70 \\
75\end{array}$ & $\begin{array}{l}10 \\
10\end{array}$ & $\begin{array}{l}925.4166 \\
951.2512\end{array}$ & $\begin{array}{l}173.6482 \\
173.6482\end{array}$ & $\begin{array}{r}\quad 366.8241 \\
\quad 254.887\end{array}$ & $\begin{array}{l}1435.889 \\
1379.786\end{array}$ \\
\hline & 80 & 10 & 969.8463 & 173.6482 & $\begin{array}{l}171.0101 \\
0\end{array}$ & $\begin{array}{r}1314.505 \\
\end{array}$ \\
\hline & $\begin{array}{l}85 \\
90\end{array}$ & $\begin{array}{l}10 \\
10\end{array}$ & $\begin{array}{l}981.0603 \\
984.8078\end{array}$ & $\begin{array}{l}173.6422 \\
13.6482\end{array}$ & $\begin{array}{l}85.83165 \\
6.03 E-14\end{array}$ & $\begin{array}{r}1240.54 \\
1158.456\end{array}$ \\
\hline & 0 & 15 & 0 & $\begin{array}{r}258.819 \\
25.819\end{array}$ & 965.9258 & 1224.745 \\
\hline & $\begin{array}{r}5 \\
10\end{array}$ & $\begin{array}{l}15 \\
15\end{array}$ & $\begin{array}{l}84.18598 \\
167.7313\end{array}$ & $\begin{array}{l}\begin{array}{l}2588.819 \\
258.819\end{array} \\
2\end{array}$ & $\begin{array}{l}992.2502 \\
951.2512\end{array}$ & $\begin{array}{l}1335.255 \\
1377.802\end{array}$ \\
\hline & 15 & 15 & & 258.819 & 933.0127 & 1441.832 \\
\hline & $\begin{array}{l}20 \\
25\end{array}$ & $\begin{array}{l}15 \\
15\end{array}$ & $\begin{array}{l}330.3661 \\
408.2179\end{array}$ & $\begin{array}{l}\begin{array}{l}2588.819 \\
258.819\end{array} \\
2\end{array}$ & $\begin{array}{r}907.6734 \\
875.4261\end{array}$ & $\begin{array}{l}1496.859 \\
1542.463\end{array}$ \\
\hline & 30 & 15 & 482.9629 & $\begin{array}{l}258.819 \\
258.819\end{array}$ & 836.5163 & $\begin{array}{l}1578.298 \\
\end{array}$ \\
\hline & 35 & $\begin{array}{l}15 \\
15\end{array}$ & & & 791.2401 & 1604.091 \\
\hline & $\begin{array}{l}40 \\
45\end{array}$ & $\begin{array}{l}15 \\
15\end{array}$ & $\begin{array}{l}620.88822 \\
683.0127\end{array}$ & $\begin{array}{l}\begin{array}{l}2588.819 \\
258.819\end{array} \\
2\end{array}$ & $\begin{array}{l}\quad 7399.9421 \\
683.0127\end{array}$ & $\begin{array}{l}16169.646 \\
1624.844\end{array}$ \\
\hline & 50 & 15 & & 258.819 & 620.8852 & 1619.646 \\
\hline & $\begin{array}{l}55 \\
60\end{array}$ & $\begin{array}{l}15 \\
15\end{array}$ & $\begin{array}{l}791.2401 \\
836.5163\end{array}$ & $\begin{array}{l}\begin{array}{l}2588.819 \\
258.819\end{array} \\
2\end{array}$ & $\begin{array}{l}554.0323 \\
482.9629\end{array}$ & $\begin{array}{l}1604.091 \\
1578.298\end{array}$ \\
\hline & 65 & 15 & 875.4261 & $\begin{array}{l}258.819 \\
258.819\end{array}$ & 408.2179 & $\begin{array}{l}1542.463 \\
\end{array}$ \\
\hline & $\begin{array}{l}70 \\
75\end{array}$ & $\begin{array}{l}15 \\
15\end{array}$ & $\begin{array}{l}907.6734 \\
933.0127\end{array}$ & $\begin{array}{l}\begin{array}{l}258.819 \\
258.819\end{array} \\
2.89\end{array}$ & $\begin{array}{r}330.3661 \\
250\end{array}$ & $\begin{array}{l}1446.859 \\
1441.832\end{array}$ \\
\hline & 80 & 15 & 951.2512 & 258.819 & 167.7313 & 1377.802 \\
\hline & 85 & $\begin{array}{l}15 \\
15\end{array}$ & $\begin{array}{r}962.2502 \\
965\end{array}$ & $\begin{array}{r}258.819 \\
25.819\end{array}$ & $\begin{array}{r}84.18598 \\
5\end{array}$ & 1305.255 \\
\hline & $\begin{array}{r}90 \\
0\end{array}$ & $\begin{array}{l}15 \\
20\end{array}$ & $\begin{array}{r}965.9258 \\
0\end{array}$ & $\begin{array}{r}2588.819 \\
342.0201\end{array}$ & $\begin{array}{rl}5 & 5.92-14-14 \\
939.6926\end{array}$ & $\begin{array}{l}1224.745 \\
1281.713\end{array}$ \\
\hline & 5 & 20 & 81.89961 & 342.0201 & 936.1168 & 1360.037 \\
\hline & $\begin{array}{l}10 \\
15\end{array}$ & $\begin{array}{l}20 \\
20\end{array}$ & $\begin{array}{l}163.1759 \\
243.210\end{array}$ & 342.0201 & 925.4166 & 1430.613 \\
\hline & $\begin{array}{l}10 \\
20\end{array}$ & 20 & $\begin{array}{l}243.2103 \\
321.3938\end{array}$ & $\begin{array}{l}342.0201 \\
342.0201\end{array}$ & $\begin{array}{l}997.6734 \\
883.0222\end{array}$ & $\begin{array}{l}1442.904 \\
1546.436\end{array}$ \\
\hline & 25 & 20 & $\begin{array}{l}397.1313 \\
469\end{array}$ & 342.0201 & $\begin{array}{l}851.6507 \\
\end{array}$ & $\begin{array}{l}1590.802 \\
162565\end{array}$ \\
\hline & $\begin{array}{l}30 \\
35\end{array}$ & $\begin{array}{l}20 \\
20\end{array}$ & $\begin{array}{l}469.8463 \\
538.9855\end{array}$ & $\begin{array}{l}342.0201 \\
342.0201\end{array}$ & $\begin{array}{l}8131.7977 \\
769.7511\end{array}$ & $\begin{array}{l}1625.604 \\
1650.757\end{array}$ \\
\hline & 40 & 20 & 604.0228 & 342.0201 & 719.8463 & 1665.889 \\
\hline & $\begin{array}{l}45 \\
50\end{array}$ & $\begin{array}{l}20 \\
20\end{array}$ & $\begin{array}{r}664.4463 \\
719.8463\end{array}$ & $\begin{array}{l}342.0201 \\
342.0201\end{array}$ & $\begin{array}{r}664.463 \\
604.0228\end{array}$ & $\begin{array}{l}16 / 0.946 \\
1665.889\end{array}$ \\
\hline & 55 & 20 & 769.7511 & 342.0201 & 538.9855 & 1650.757 \\
\hline & $\begin{array}{l}60 \\
65\end{array}$ & 20 & $\begin{array}{l}813.7977 \\
851.650\end{array}$ & 342.0201 & $\begin{array}{l}469.8463 \\
3\end{array}$ & 1625.664 \\
\hline & $\begin{array}{l}65 \\
70\end{array}$ & 20 & $\begin{array}{l}851.6507 \\
883.0220\end{array}$ & $\begin{array}{l}342.0201 \\
3420\end{array}$ & $39 / .13938$ & 1590.8026 \\
\hline & 75 & 20 & 907.6734 & 342.0201 & 243.2103 & $\begin{array}{l}1492.900 \\
1492.950\end{array}$ \\
\hline & 80 & 20 & 925.4166 & 342.0201 & 3.1759 & 1430.613 \\
\hline & 85 & 20 & $\begin{array}{r}936.1168 \\
9396926\end{array}$ & $\begin{array}{l}342.0201 \\
3420201\end{array}$ & $\begin{array}{l}81.89961 \\
5.6 F-14\end{array}$ & $\begin{array}{l}1360.037 \\
1281713\end{array}$ \\
\hline
\end{tabular}


Table 33. Tumbling Satellite Panel Area Calculations (cont'd)

\begin{tabular}{|c|c|c|c|c|c|c|c|c|c|c|c|}
\hline 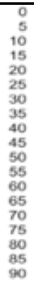 & $\begin{array}{l}20.00 \\
20.00 \\
20.00 \\
20.00 \\
20.00 \\
20.00 \\
20.00 \\
20.00 \\
20.00 \\
20.00 \\
20.00 \\
20.00 \\
20.00 \\
20.00 \\
20.00 \\
20.00 \\
20.00 \\
20.00 \\
20.00 \\
20.00\end{array}$ & 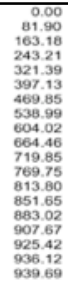 & 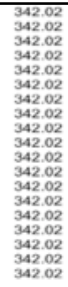 & 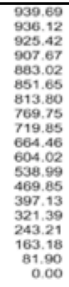 & 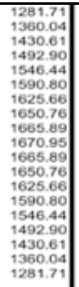 & 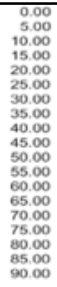 & $\begin{array}{l}70.00 \\
70.00 \\
70.00 \\
70.00 \\
70.00 \\
70.00 \\
70.00 \\
70.00 \\
70.00 \\
70.00 \\
70.00 \\
70.00 \\
70.00 \\
70.00 \\
70.00 \\
70.00 \\
70.00 \\
70.00 \\
70.00 \\
70.00 \\
70.00\end{array}$ & 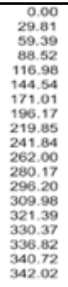 & $\begin{array}{l}939.69 \\
939.69 \\
939.69 \\
939.69 \\
939.69 \\
939.69 \\
939.69 \\
939.69 \\
939.69 \\
939.69 \\
939.69 \\
939.69 \\
939.69 \\
939.69 \\
939.69 \\
939.69 \\
939.69 \\
939.69 \\
939.69 \\
939.69\end{array}$ & 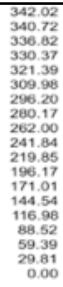 & 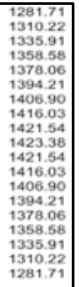 \\
\hline $\begin{array}{l}0 \\
5 \\
10 \\
15 \\
20 \\
25 \\
30 \\
35 \\
40 \\
45 \\
50 \\
55 \\
60 \\
65 \\
70 \\
75 \\
70 \\
95 \\
90\end{array}$ & $\begin{array}{l}25.00 \\
25.00 \\
25.00 \\
25.00 \\
25.00 \\
25.00 \\
25.00 \\
25.00 \\
25.00 \\
25.00 \\
25.00 \\
25.00 \\
25.00 \\
25.00 \\
25.00 \\
25.00 \\
25.00 \\
25.00 \\
25.00\end{array}$ & 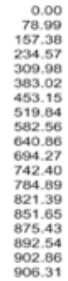 & 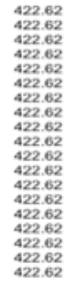 & 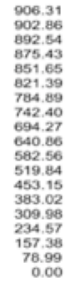 & 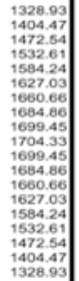 & 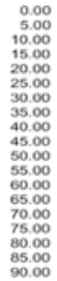 & $\begin{array}{l}75.00 \\
75.00 \\
75.00 \\
75.00 \\
75.00 \\
75.00 \\
75.00 \\
75.00 \\
75.00 \\
75.00 \\
75.00 \\
75.00 \\
75.00 \\
755.00 \\
75.00 \\
75.00 \\
75.00 \\
75.00 \\
75.00 \\
75.00\end{array}$ & 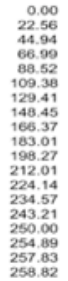 & $\begin{array}{l}965.93 \\
9655 \\
965.93 \\
9655 \\
965.93 \\
965.93 \\
9655 \\
9653 \\
965.93 \\
965.93 \\
965.93 \\
965.93 \\
965.93 \\
9655 \\
965.93 \\
965.93 \\
965.93 \\
965.93 \\
965.93 \\
965.93 \\
965.93 \\
965.93\end{array}$ & 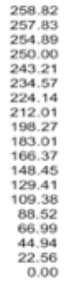 & 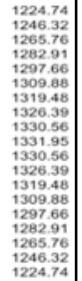 \\
\hline $\begin{array}{l}0 \\
5 \\
10 \\
15 \\
20 \\
25 \\
30 \\
35 \\
40 \\
45 \\
50 \\
55 \\
60 \\
65 \\
70 \\
75 \\
750 \\
35 \\
90\end{array}$ & $\begin{array}{l}30.00 \\
30.00 \\
30.00 \\
30.00 \\
30.00 \\
30.00 \\
30.00 \\
30.00 \\
30.00 \\
30.00 \\
30.00 \\
30.00 \\
30.00 \\
30.00 \\
30.00 \\
30.00 \\
30.00 \\
30.00 \\
30.00\end{array}$ & 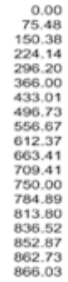 & 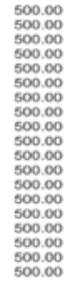 & 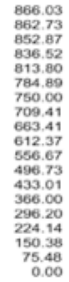 & 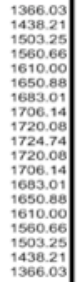 & 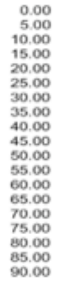 & $\begin{array}{l}80.00 \\
80.00 \\
80.00 \\
80.00 \\
60.00 \\
80.00 \\
80.00 \\
80.00 \\
80.00 \\
80.00 \\
80.00 \\
80.00 \\
80.00 \\
80.00 \\
80.00 \\
80.00 \\
80.00 \\
80.00 \\
80.00 \\
80.00\end{array}$ & $\begin{array}{r}0.00 \\
15.13 \\
30.15 \\
44.94 \\
59.99 \\
73.39 \\
73.39 \\
96.82 \\
99.60 \\
111.62 \\
122.79 \\
133.02 \\
142.24 \\
192.38 \\
157.38 \\
163.18 \\
167.73 \\
177.01 \\
172.99 \\
173.65\end{array}$ & 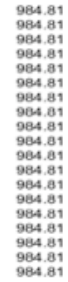 & 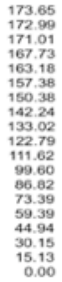 & 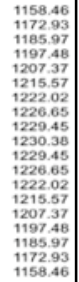 \\
\hline $\begin{array}{l}0 \\
5 \\
10 \\
150 \\
20 \\
25 \\
30 \\
35 \\
40 \\
45 \\
50 \\
55 \\
60 \\
65 \\
70 \\
75 \\
70 \\
35 \\
90\end{array}$ & $\begin{array}{l}35.00 \\
35.00 \\
35.00 \\
35.00 \\
35.00 \\
35.00 \\
35.00 \\
35.00 \\
35.00 \\
35.00 \\
35.00 \\
35.00 \\
35.00 \\
35.00 \\
35.00 \\
35.00 \\
35.00 \\
35.00 \\
35.00\end{array}$ & 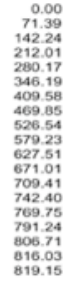 & 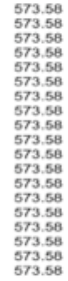 & 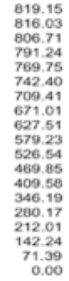 &  & 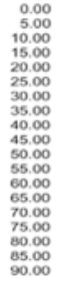 & $\begin{array}{l}85.00 \\
85.00 \\
85.00 \\
85.00 \\
85.00 \\
85.00 \\
85.00 \\
85.00 \\
85.00 \\
85.00 \\
85.00 \\
85.00 \\
85.00 \\
85.00 \\
85.00 \\
85.00 \\
85.00 \\
85.00 \\
85.00 \\
85.00\end{array}$ & $\begin{array}{r}0.00 \\
7.60 \\
15.13 \\
22.56 \\
29.81 \\
36.83 \\
36.83 \\
43.58 \\
49.99 \\
56.02 \\
61.63 \\
66.67 \\
66.77 \\
71.39 \\
75.48 \\
78.99 \\
81.90 \\
84.9 \\
84.19 \\
85.83 \\
86.02 \\
87.16\end{array}$ & 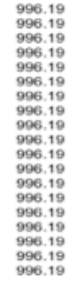 & 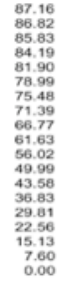 & 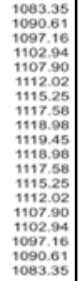 \\
\hline $\begin{array}{l}0 \\
5 \\
10 \\
15 \\
20 \\
25 \\
30 \\
35 \\
40 \\
45 \\
50 \\
55 \\
60 \\
65 \\
70 \\
75 \\
70 \\
95 \\
95 \\
90\end{array}$ & $\begin{array}{l}40.00 \\
40.00 \\
40.00 \\
40.00 \\
40.00 \\
40.00 \\
40.00 \\
40.00 \\
40.00 \\
40.00 \\
40.00 \\
40.00 \\
40.00 \\
40.00 \\
40.00 \\
40.00 \\
40.00 \\
40.00 \\
40.00 \\
40.00\end{array}$ & 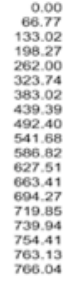 & 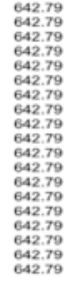 & 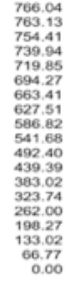 & 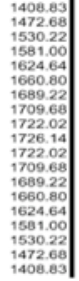 & 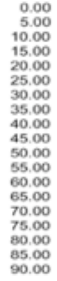 & $\begin{array}{l}90.00 \\
90.00 \\
90.00 \\
90.00 \\
90.00 \\
90.00 \\
90.00 \\
90.00 \\
90.00 \\
90.00 \\
90.00 \\
90.00 \\
90.00 \\
90.00 \\
90.00 \\
90.00 \\
90.00 \\
90.00 \\
90.00 \\
90.00 \\
90.00\end{array}$ & $\begin{array}{l}0.00 \\
0.00 \\
0.00 \\
0.00 \\
0.00 \\
0.00 \\
0.00 \\
0.00 \\
0.00 \\
0.00 \\
0.00 \\
0.00 \\
0.00 \\
0.00 \\
0.00 \\
0.00 \\
0.00 \\
0.00 \\
0.00\end{array}$ & $\begin{array}{l}10000.00 \\
1000.00 \\
1000.00 \\
1000.00 \\
1000.00 \\
10000.00 \\
1000.00 \\
1000.00 \\
1000.00 \\
1000.00 \\
10000.00 \\
1000.00 \\
1000.00 \\
1000.00 \\
10000.00 \\
1000.00 \\
1000.00 \\
1000.00 \\
1000.00 \\
1000.00\end{array}$ & $\begin{array}{l}0.00 \\
0.00 \\
0.00 \\
0.00 \\
0.00 \\
0.00 \\
0.00 \\
0.00 \\
0.00 \\
0.00 \\
0.00 \\
0.00 \\
0.00 \\
0.00 \\
0.00 \\
0.00 \\
0.00 \\
0.00 \\
0.00 \\
0.00\end{array}$ & 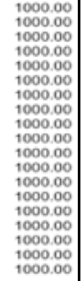 \\
\hline $\begin{aligned} 0 \\
5 \\
10 \\
15 \\
20 \\
25 \\
30 \\
35 \\
40 \\
45 \\
50 \\
55 \\
60 \\
65 \\
70 \\
75 \\
70 \\
95 \\
90\end{aligned}$ & $\begin{array}{l}45.00 \\
45.00 \\
45.00 \\
45.00 \\
45.00 \\
45.00 \\
45.00 \\
45.00 \\
45.00 \\
45.00 \\
45.00 \\
45.00 \\
45.00 \\
4500 \\
45.00 \\
45.00 \\
45.00 \\
45.00 \\
45.00\end{array}$ & 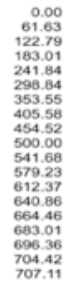 & $\begin{array}{l}707.11 \\
707.11 \\
707.11 \\
707.11 \\
707.11 \\
707.11 \\
707.11 \\
707.11 \\
707.11 \\
707.11 \\
707.11 \\
707.11 \\
707.11 \\
707.11 \\
707.11 \\
707.11 \\
707.11 \\
707.11 \\
707.11 \\
707.11 \\
707.11 \\
707.11 \\
707.11\end{array}$ & 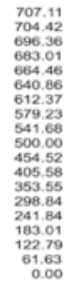 & 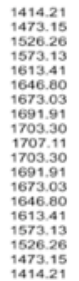 & & & & & & \\
\hline
\end{tabular}




\section{Appendix H. Thermal Equilibrium Worst-Case Hot/Cold}

Using the equations from section 3.2.3 several calculations were run evaluating multiple heating conditions with multiple environmental conditions over both worst-case cold and worst-case hot scenarios. A sample calculation is shown below for a worst-case cold scenario.

$$
Q_{\text {EarthIR }}+Q_{\text {Internal }}=\varepsilon_{\text {Satellite }} \sigma \mathrm{A}\left(T_{\text {Satellite }}^{4}-T_{\text {Space }}^{4}\right)
$$

\section{Equation 22. Radiative Exchange Equation}

Plugging in the Earthshine value, the surface area of the satellite, the StefanBoltzman constant, the temperature of space (effectively zero) and the emissivity value for the spacecraft gives the next equation.

$$
218 \frac{W}{m^{2}} * .84 * .05 m^{2}+1.06 W=.84 *\left(\frac{5.67 \times 10^{-8} W}{m^{2} K^{4}}\right) * .06 m^{2}\left(T_{\text {Satellite }}^{4}-0\right)
$$

\section{Equation 23. Radiative Exchange Equation with Values}

$$
\begin{aligned}
& \text { Solving for the temperature of the satellite gives the following equation. } \\
& {\left[\frac{\left(218 \frac{W}{m^{2}} * .84 * .05 m^{2}+1.06 W\right)}{.84 *\left(\frac{5.67 \times 10^{-8} W}{m^{2} K^{4}}\right) * .06 m^{2}}\right]^{1 / 4}=\left(T_{\text {Satellite }}\right)}
\end{aligned}
$$

Equation 24. Radiative Equation Solved for Satellite Temperature

For this case the temperature of the satellite was found to be $-29^{\circ} \mathrm{C}$. 
Table 34. Inputs for Thermal Equilibrium Calculations

\begin{tabular}{|c|c|c|c|c|c|c|c|}
\hline & Absorptivity & Emissivity & Q solar & Q Albedo & Q IR & Internal Power & Eclipse Factor \\
\hline CASE 1 - Initial & 0.92 & 0.85 & 0 & 0 & 218 & 1 & 0 \\
\hline CASE $2 \mathrm{a}$ - Cold w/o Adjustments & 0.87 & 0.84 & 0 & 0 & 218 & 1.0615 & 0 \\
\hline CASE 2b - Cold w/ Adjustments & 0.87 & 0.84 & 0 & 0 & 218 & 1.0615 & 0 \\
\hline CASE 2c - Cold w/ Single Face (Gilmore) & 0.87 & 0.84 & 0 & 0 & 218 & 1.0615 & 0 \\
\hline CASE 3 - Mode 1 (Standard Operations) & 0.87 & 0.84 & 0 & 0 & 218 & 1.0615 & 0 \\
\hline CASE 3 - Mode 2a. (Inc. Beacon Rate - 90s) & 0.87 & 0.84 & 0 & 0 & 218 & 1.2859 & 0 \\
\hline CASE 3 - Mode 2b. (Inc. Beacon Rate - 60s) & 0.87 & 0.84 & 0 & 0 & 218 & 1.71622 & 0 \\
\hline CASE 3 - Mode 3. (Payload Enabled - ON) & 0.87 & 0.84 & 0 & 0 & 218 & 1.77947 & 0 \\
\hline CASE 3 - Mode 4a. (ADC ON) & 0.87 & 0.84 & 0 & 0 & 218 & 5.09652 & 0 \\
\hline CASE 3 - Mode 4b. (ADC ON \& Beacon Inc. - 90s) & 0.87 & 0.84 & 0 & 0 & 218 & 5.32092 & 0 \\
\hline CASE 3 - Mode 4c. (ADC ON \& Beacon Inc. - 60s) & 0.87 & 0.84 & 0 & 0 & 218 & 5.74552 & 0 \\
\hline CASE 3 - Mode 5. (Increased "Data Drop") & 0.87 & 0.84 & 0 & 0 & 218 & 3.38712 & 0 \\
\hline CASE 4a - Hot w/o Adjustments & 0.87 & 0.84 & 1414 & 805.98 & 244 & 1.0615 & 1 \\
\hline CASE $4 \mathrm{~b}$ - Hot w/ Adjustments & 0.87 & 0.84 & 1414 & 805.98 & 244 & 1.0615 & 1 \\
\hline CASE 4c - Hot w/ Single Face (Gilmore) & 0.87 & 0.84 & 1414 & 805.98 & 244 & 1.0615 & 1 \\
\hline
\end{tabular}

Table 35. Outputs for Thermal Equilibrium Calculations

\begin{tabular}{|l|r|r|r|r|r|r|r|}
\hline & Earthshine A & Solar A & Albedo & Stefan Boltzman & Q external & Adjusted (E\&A) & Q total \\
\hline CASE 1 - & 0.06 & 0 & 0 & $5.67 \mathrm{E}-08$ & 11.182092 & 12.182092 & 13.182092 \\
\hline CASE 2a & 0.06 & 0.0173 & 0.0173 & $5.67 \mathrm{E}-08$ & 10.9872 & 12.0487 & 13.1102 \\
\hline CASE 2b & 0.05 & 0.0149 & 0.0149 & $5.67 \mathrm{E}-08$ & 9.156 & 10.2175 & 11.279 \\
\hline CASE 2c & 0.01 & 0.0149 & 0.0149 & $5.67 \mathrm{E}-08$ & 1.8312 & 2.8927 & 3.9542 \\
\hline CASE 3 - & 0.05 & 0.0149 & 0.0149 & $5.67 \mathrm{E}-08$ & 9.156 & 10.2175 & 11.279 \\
\hline CASE 3 - & 0.05 & 0.0149 & 0.0149 & $5.67 \mathrm{E}-08$ & 9.156 & 10.4419 & 11.7278 \\
\hline CASE 3 - & 0.05 & 0.0149 & 0.0149 & $5.67 \mathrm{E}-08$ & 9.156 & 10.87222 & 12.58844 \\
\hline CASE 3 - & 0.05 & 0.0149 & 0.0149 & $5.67 \mathrm{E}-08$ & 9.156 & 10.93547 & 12.71494 \\
\hline CASE 3 - & 0.05 & 0.0149 & 0.0149 & $5.67 \mathrm{E}-08$ & 9.156 & 14.25252 & 19.34904 \\
\hline CASE 3 - & 0.05 & 0.0149 & 0.0149 & $5.67 \mathrm{E}-08$ & 9.156 & 14.47692 & 19.79784 \\
\hline CASE 3 - & 0.05 & 0.0149 & 0.0149 & $5.67 \mathrm{E}-08$ & 9.156 & 14.90152 & 20.64704 \\
\hline CASE 3 - & 0.05 & 0.0149 & 0.0149 & $5.67 \mathrm{E}-08$ & 9.156 & 12.54312 & 15.93024 \\
\hline CASE 4a & 0.05 & 0.0173 & 0.0173 & $5.67 \mathrm{E}-08$ & 43.660919 & 44.72241898 & 45.783919 \\
\hline CASE 4b & 0.05 & 0.0149 & 0.0149 & $5.67 \mathrm{E}-08$ & 39.0256007 & 40.08710074 & 41.1486007 \\
\hline CASE 4c & 0.01 & 0.01 & 0.01 & $5.67 \mathrm{E}-08$ & 21.363426 & 22.424926 & 23.486426 \\
\hline
\end{tabular}


Table 36. Thermal Equilibrium Calculation Results

\begin{tabular}{|l|c|c|}
\hline & Solved for T Kelvin & (Celsius) \\
\hline CASE 1 - Initial & 259 & -14 \\
\hline CASE 2a - Cold w/o Adjustments & 260 & -13 \\
\hline CASE 2b - Cold w/ Adjustments & 241 & -32 \\
\hline CASE 2c - Cold w/ Single Face (Gilmore) & 205 & -68 \\
\hline CASE 3 - Mode 1 (Standard Operations) & 241 & -32 \\
\hline CASE 3 - Mode 2a. (Inc. Beacon Rate - 90s) & 253 & -20 \\
\hline CASE 3 - Mode 2b. (Inc. Beacon Rate - 60s) & 258 & -15 \\
\hline CASE 3 - Mode 3. (Payload Enabled - ON) & 258 & -15 \\
\hline CASE 3 - Mode 4a. (ADC ON) & 287 & 14 \\
\hline CASE 3 - Mode 4b. (ADC ON \& Beacon Inc. - 90s) & 289 & 16 \\
\hline CASE 3 - Mode 4c. (ADC ON \& Beacon Inc. - 60s) & 292 & 19 \\
\hline CASE 3 - Mode 5. (Increased "Data Drop") & 273 & 0 \\
\hline CASE 4a - Hot w/o Adjustments & 356 & 83 \\
\hline CASE 4b - Hot w/ Adjustments & 346 & 73 \\
\hline CASE 4c - Hot w/ Single Face (Gilmore) & 315 & 42 \\
\hline
\end{tabular}

Table 37. Adjusted "Worst-Case” Hot w/ Adjusted Internal Heat Flux

\begin{tabular}{|l|c|c|}
\hline & Solved for T Kelvin & (Celsius) \\
\hline CASE 5-2a - Hot w/ Adjustments & 347 & 74 \\
\hline CASE 5-2b - Hot w/ Adjustments & 349 & 76 \\
\hline CASE 5-3 - Hot w/ Adjustments & 349 & 76 \\
\hline CASE 5-4a - Hot w/ Adjustments & 362 & 89 \\
\hline CASE 5-4b - Hot w/ Adjustments & 363 & 90 \\
\hline CASE 5-4c - Hot w/ Adjustments & 365 & 92 \\
\hline CASE 5-5 - Hot w/ Adjustments & 356 & 83 \\
\hline
\end{tabular}




\section{APPENDIX I. THERMAL RESISTANCE CALCULATIONS}

Using the equations in section 3.2.5 and applying the physical properties of the satellite and it's materials gives the equation below.

$$
R_{E Q}=\frac{.025 m}{\frac{18 W}{m K} \cdot 00014 m^{2}}+\frac{\frac{3.79 K}{W}}{4}+\frac{.1 m}{\frac{128 W}{m K} * .001 m^{2}}+\frac{\frac{3.79 K}{W}}{4}+\frac{.025 m}{\frac{18 W}{m K} .00014 m^{2}}
$$

Equation 25. $\boldsymbol{R}_{E Q}$ from Side Panel to Side Panel (Lateral) with Fourier's Substitution -

Solved

Solving this equation gave a total resistance of $23.3 \mathrm{~K} / \mathrm{W}$. Figure 99 below was used to calculate the view factor between parallel panels inside the satellite.

With the values of beta and alpha at 8.5 and 7.6 respectively the view factor was found to be approximately .85 between the central PCBs of the CP3 CubeSat.

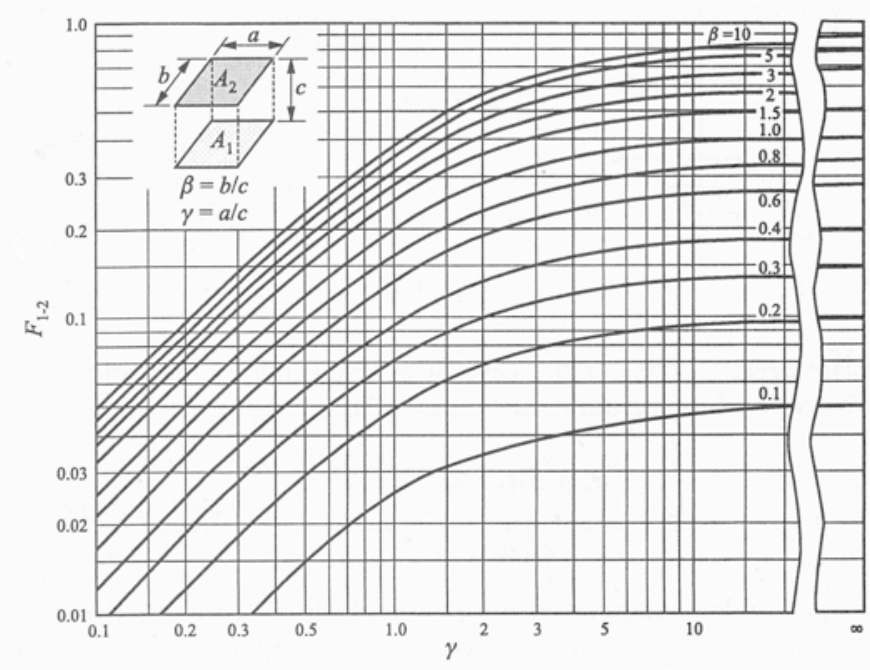

Figure 97 Parallel Flat Plates View Factor (Configuration Factor) ${ }^{31}$ 


\section{APPENDIX J. TSS and SINDA Modeling}

The figures below illustrate the method for input into TSS and the resulting data, plotted, from the SINDA post-processor. Shown in Figure 103 is the orbital heating input generated by TSS. The satellite was modeled as a simple sphere with the equivalent surface area as a $10 \times 10 \times 10 \mathrm{~cm}$ CubeSat. The parameters for emissivity and absorptivity were directly from the calculations in Appendix 1. The orbit used was the DNEPR mission orbit for CP3. The internal heat generation settings were all specified per the Appendix 2 measured and calculated values (orbital average by duty cycle). Heating duty cycles were applied using the .tsshr file specified.

The one assumption made in the SINDA modeling was giving the satellite the conductivity of aluminum. For the purposes of a single node simulation this is a non-issue, but a multi-node simulation would require adequate conductivity information in order to accurately model the difference between nodes. 


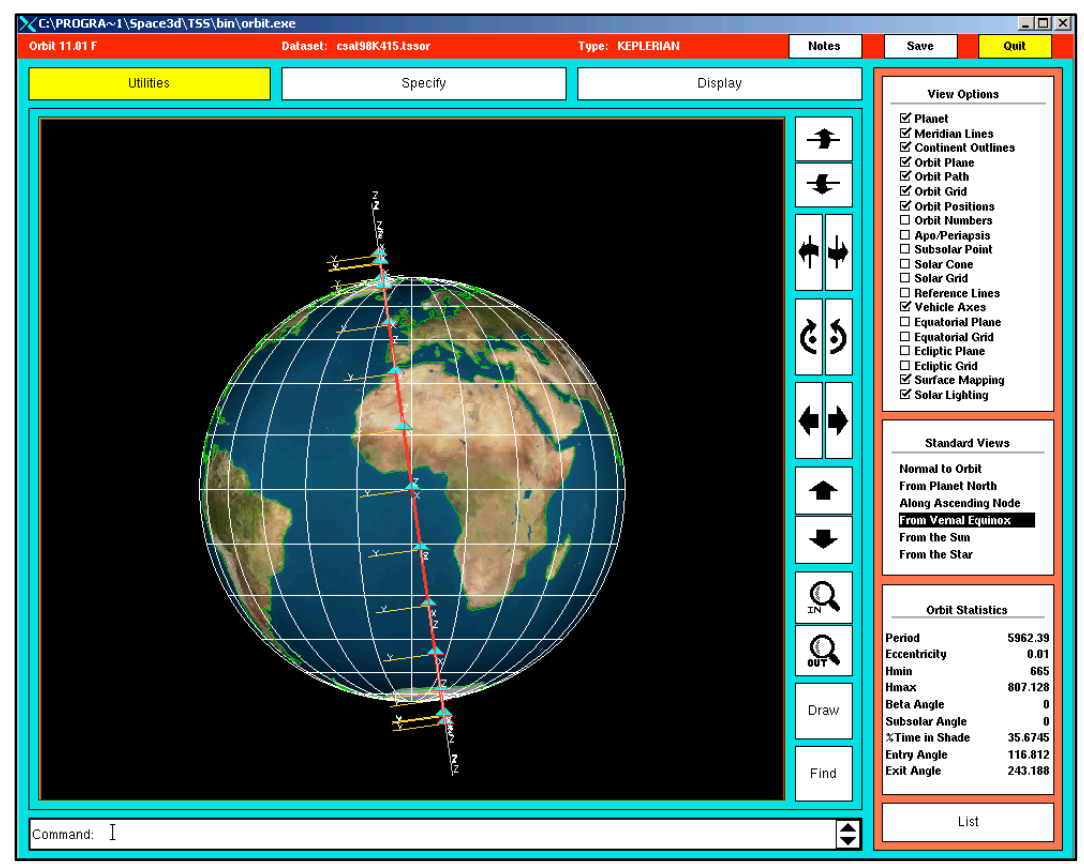

Figure 98. TSS Orbital Input Window and Heating Propagation

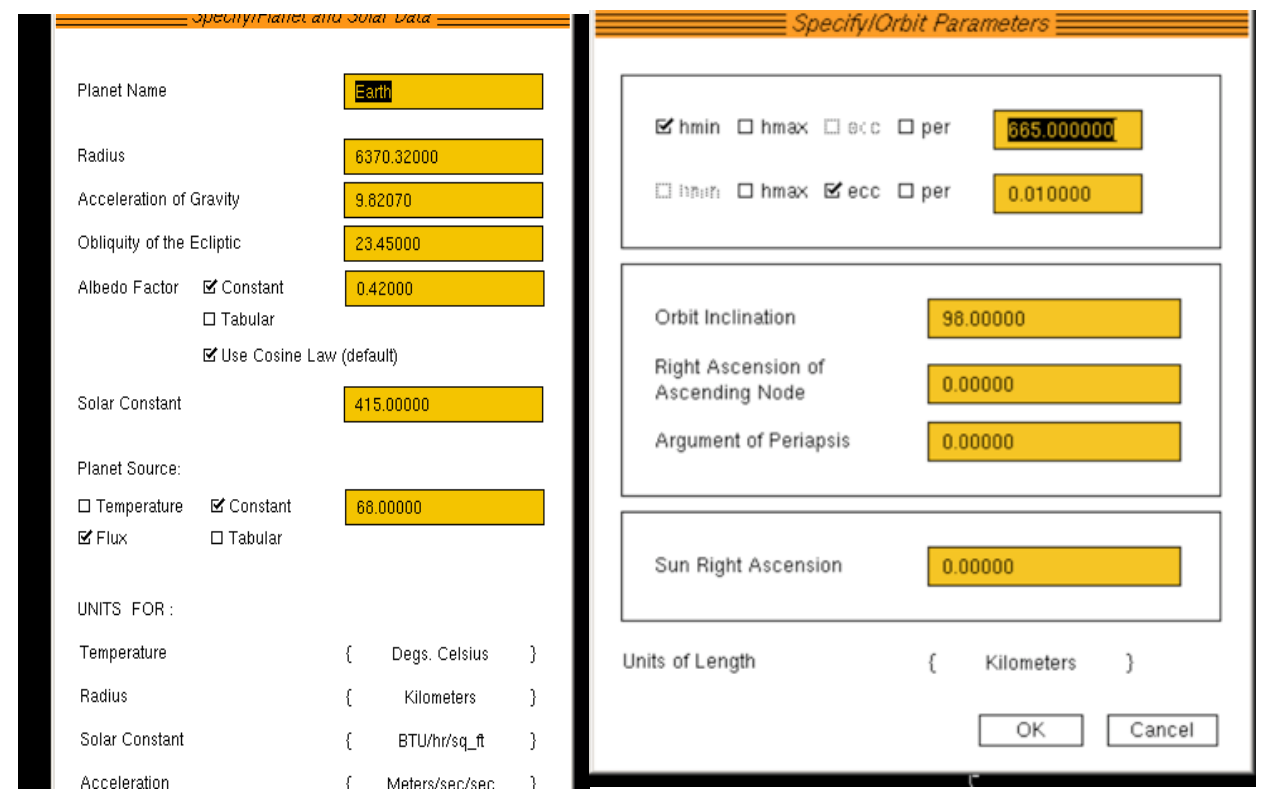

Figure 99. TSS Variables Input 

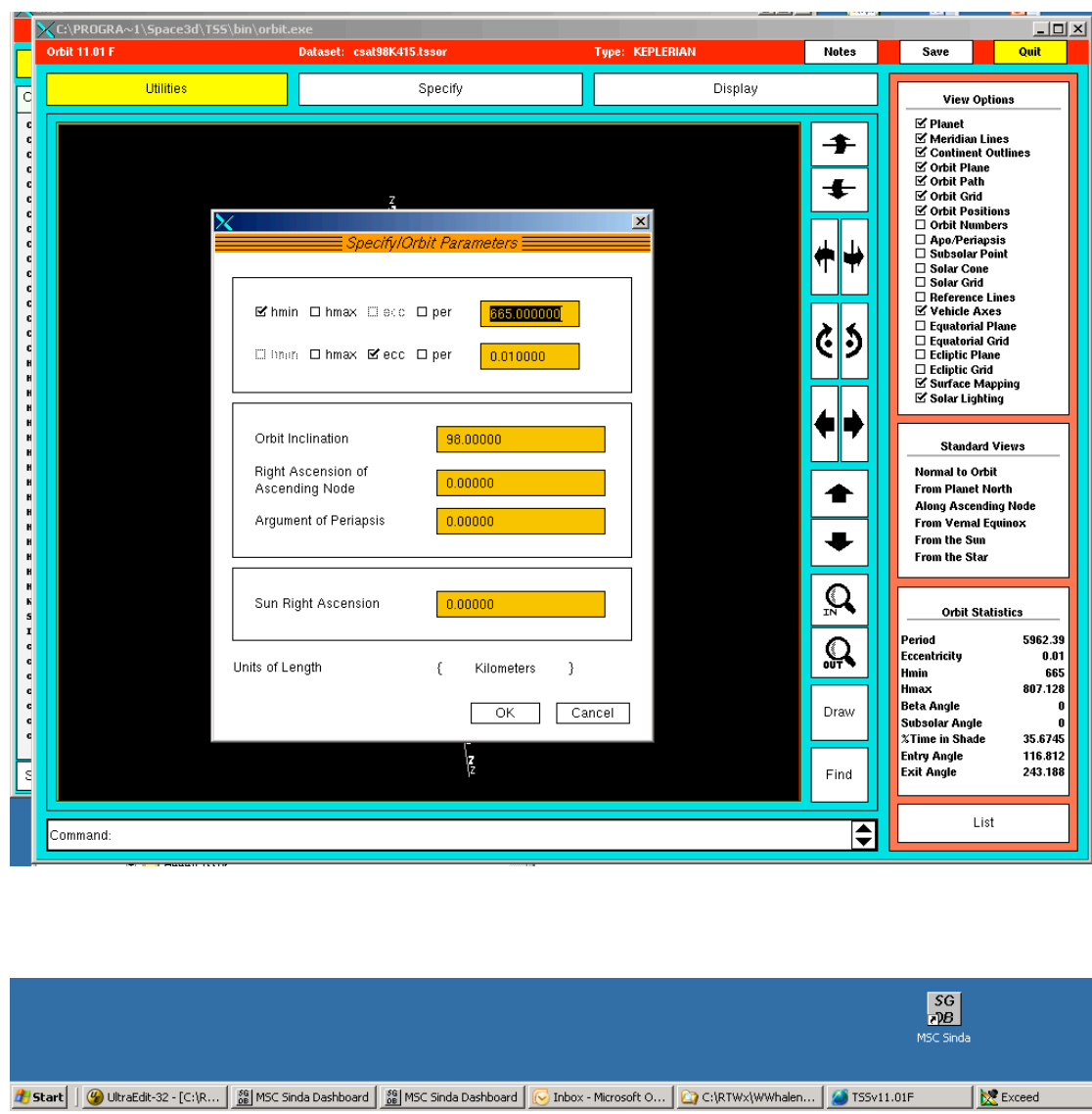

Figure 100. TSS Variables Input Cont'd

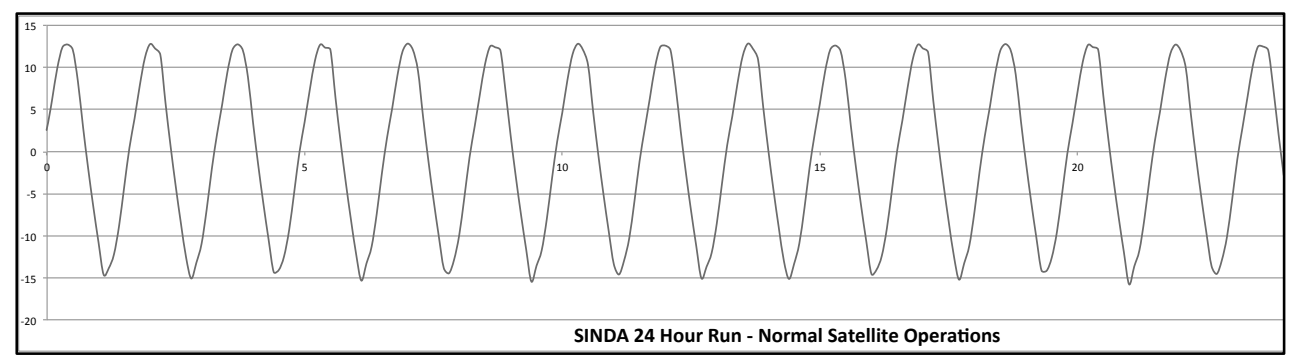

Figure 101. SINDA Output - 24 Hour Nominal (Standard Operations) 


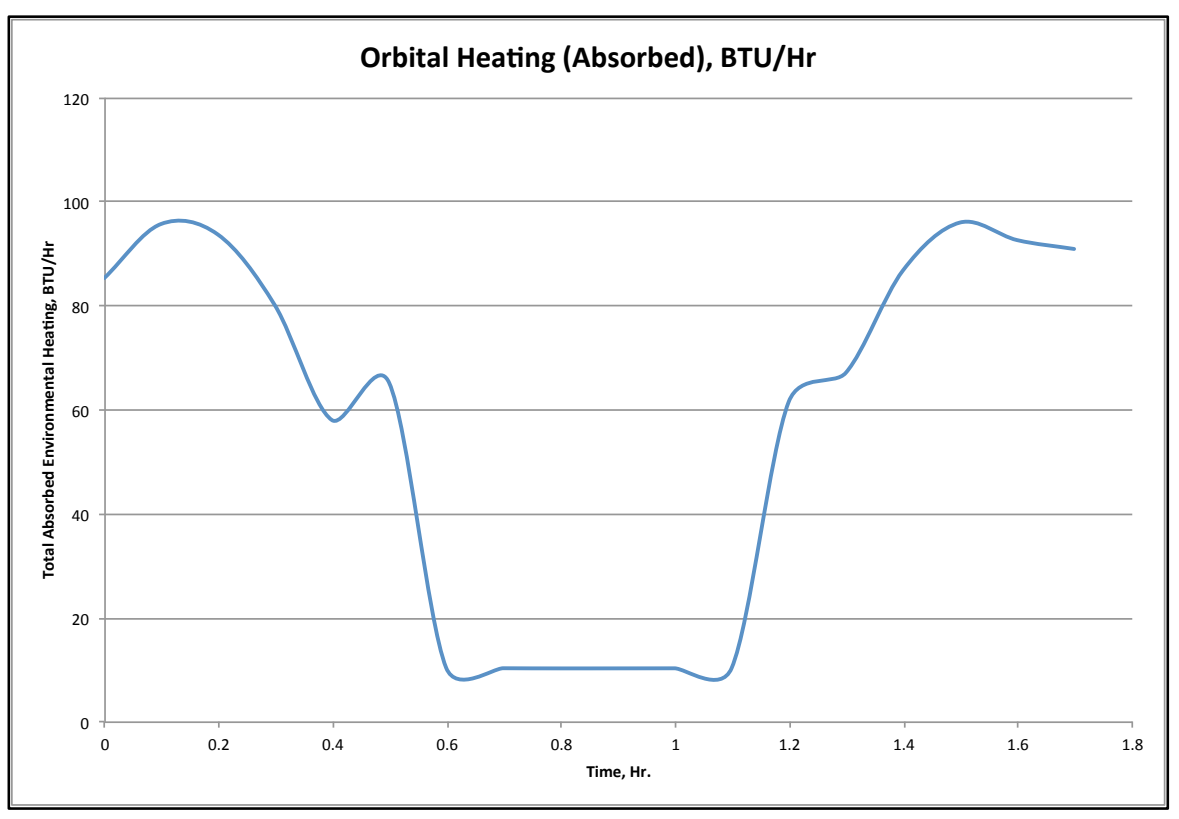

Figure 102. SINDA Orbital Heating for One Orbit

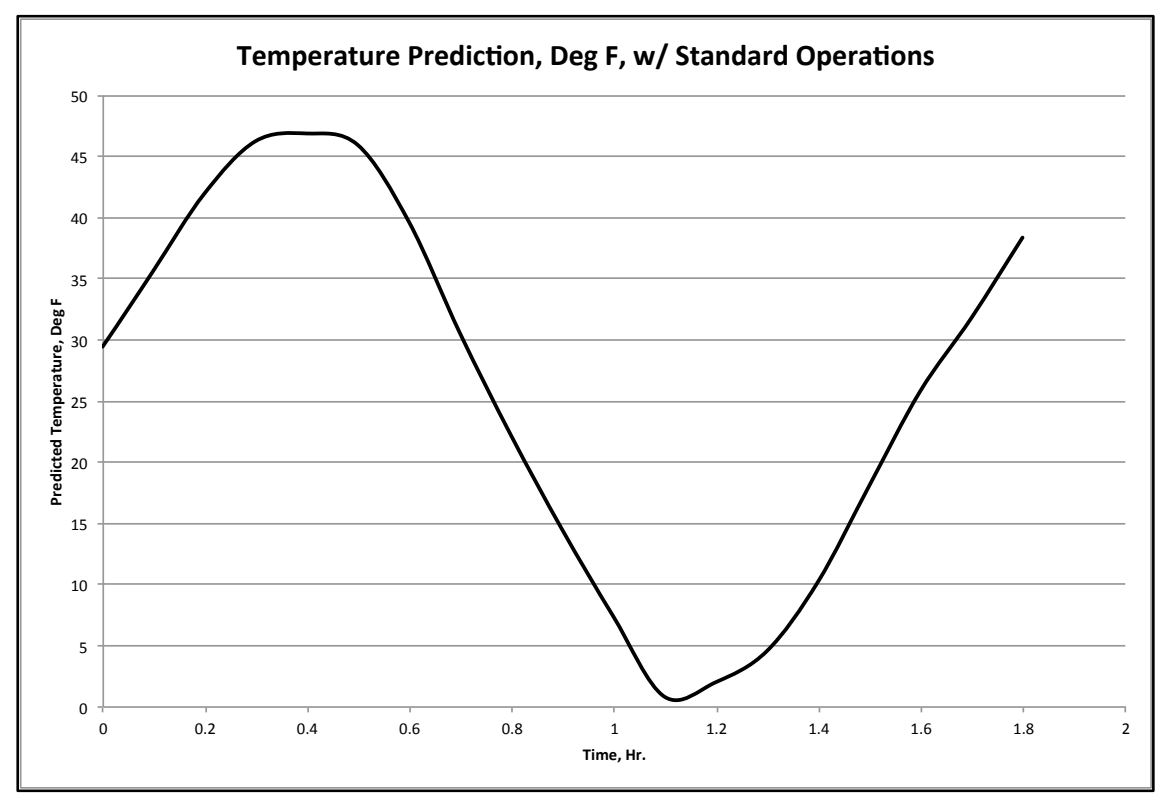

Figure 103. SINDA Output Mode 1 Standard Operations 


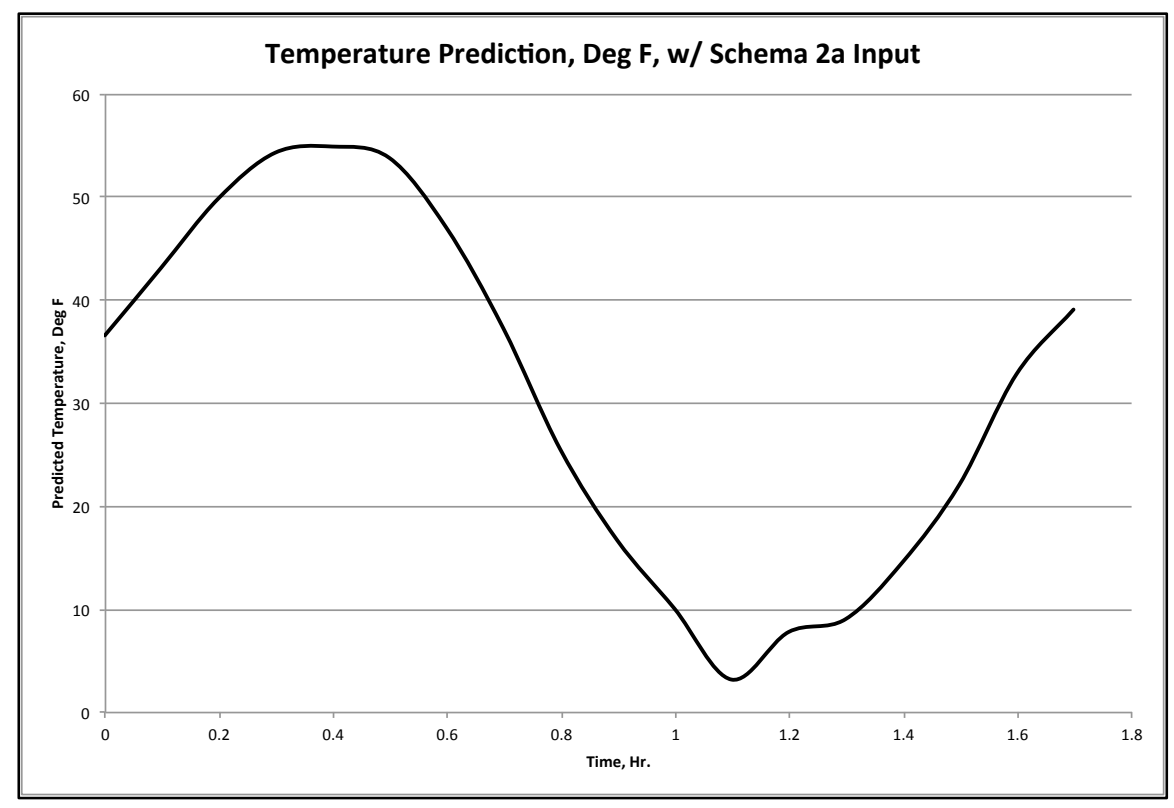

Figure 104. SINDA Output Mode 2a Increased Beacon Rate

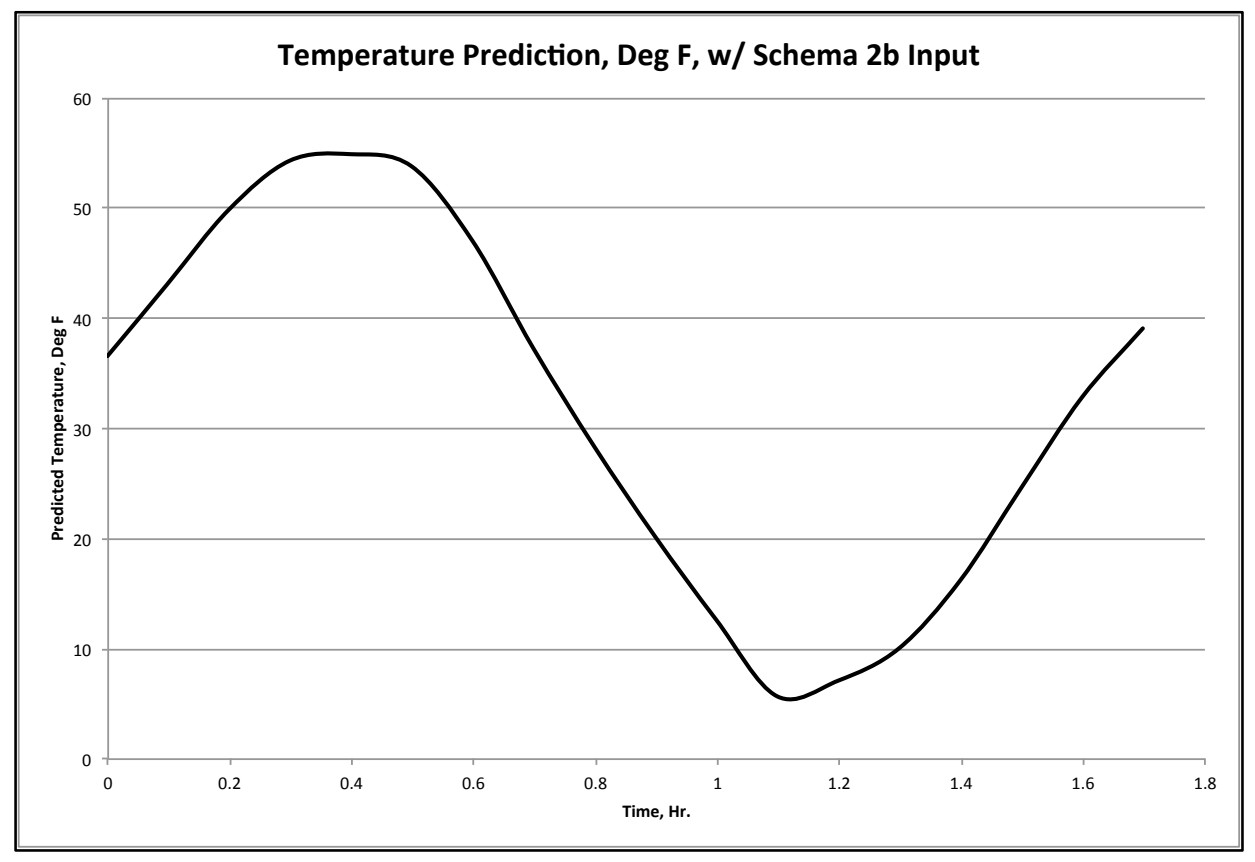

Figure 105. SINDA Output w/ High Beacon Rate \& High Sensor Snap Rate (Schema 2b) 


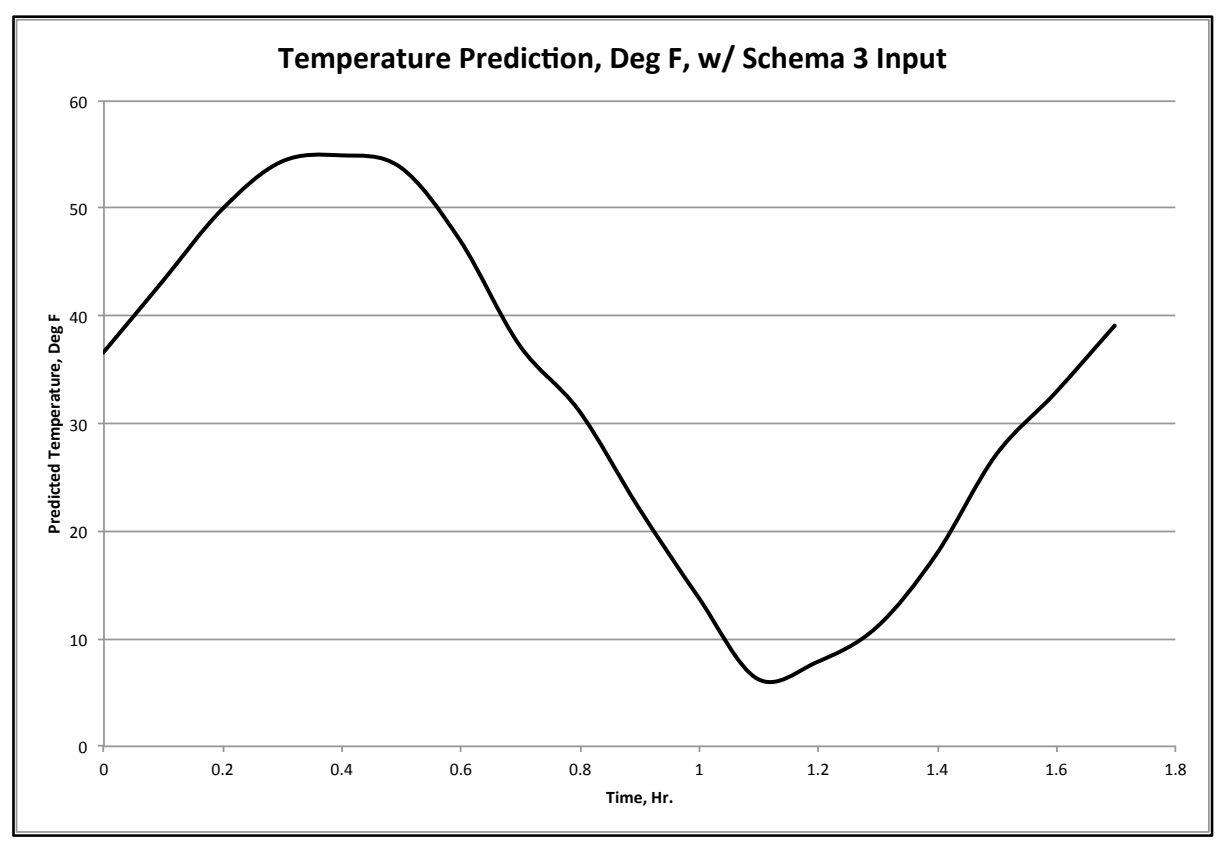

Figure 106. SINDA Output w/ Payload Enabled (Schema 3)

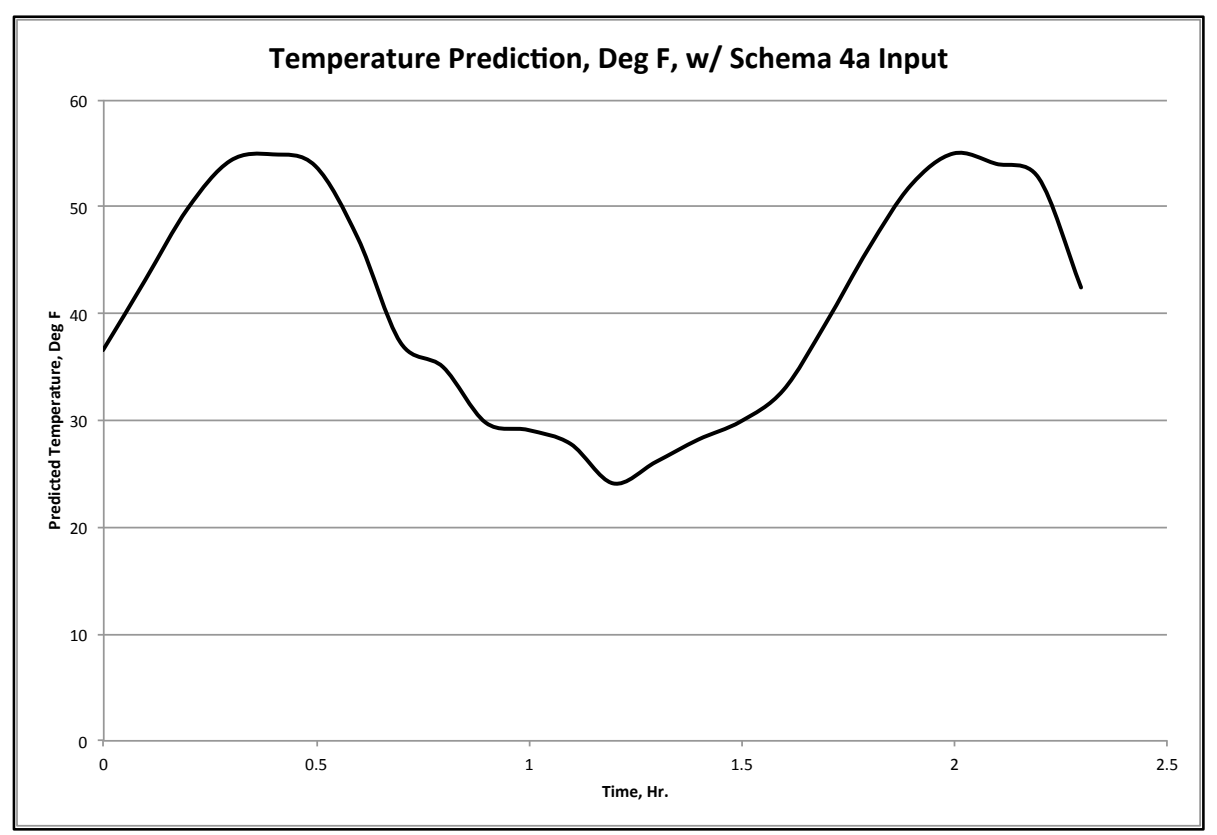

Figure 107. SINDA Output w/ ADC On (Schema 4a) 


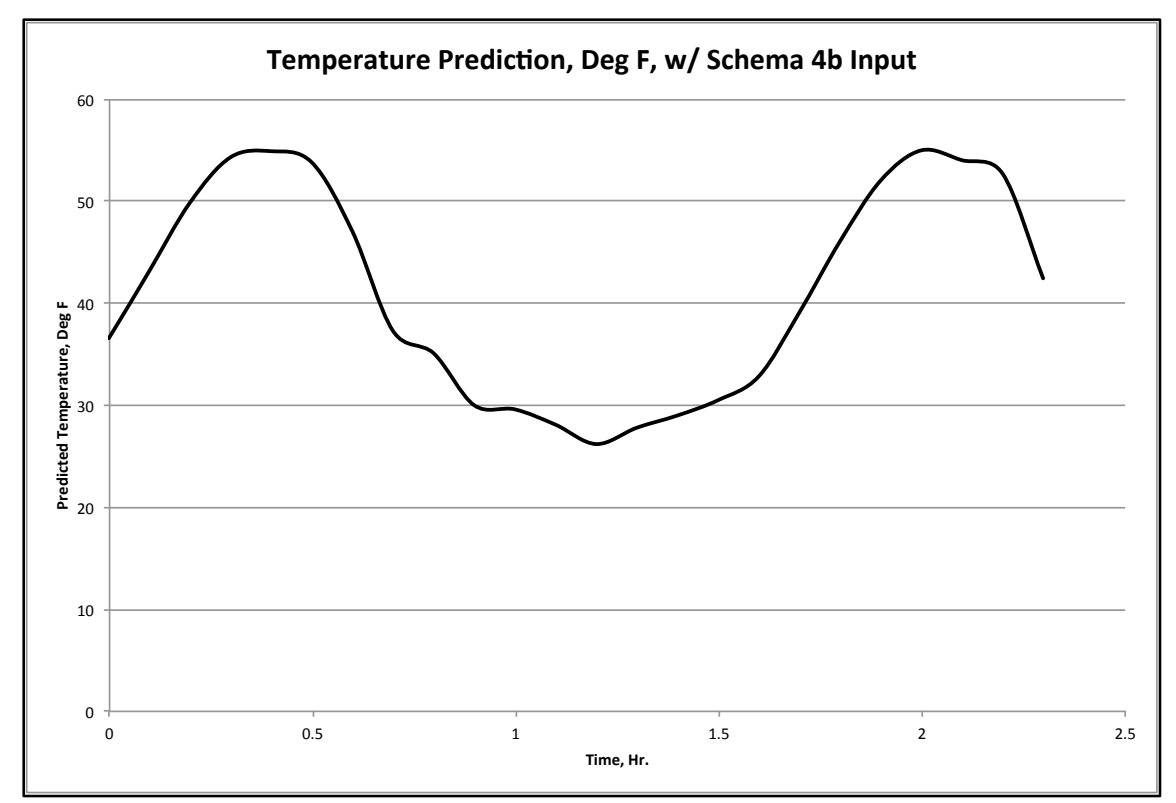

Figure 108. SINDA Output w/ ADC On \& 90s (Schema 4b)

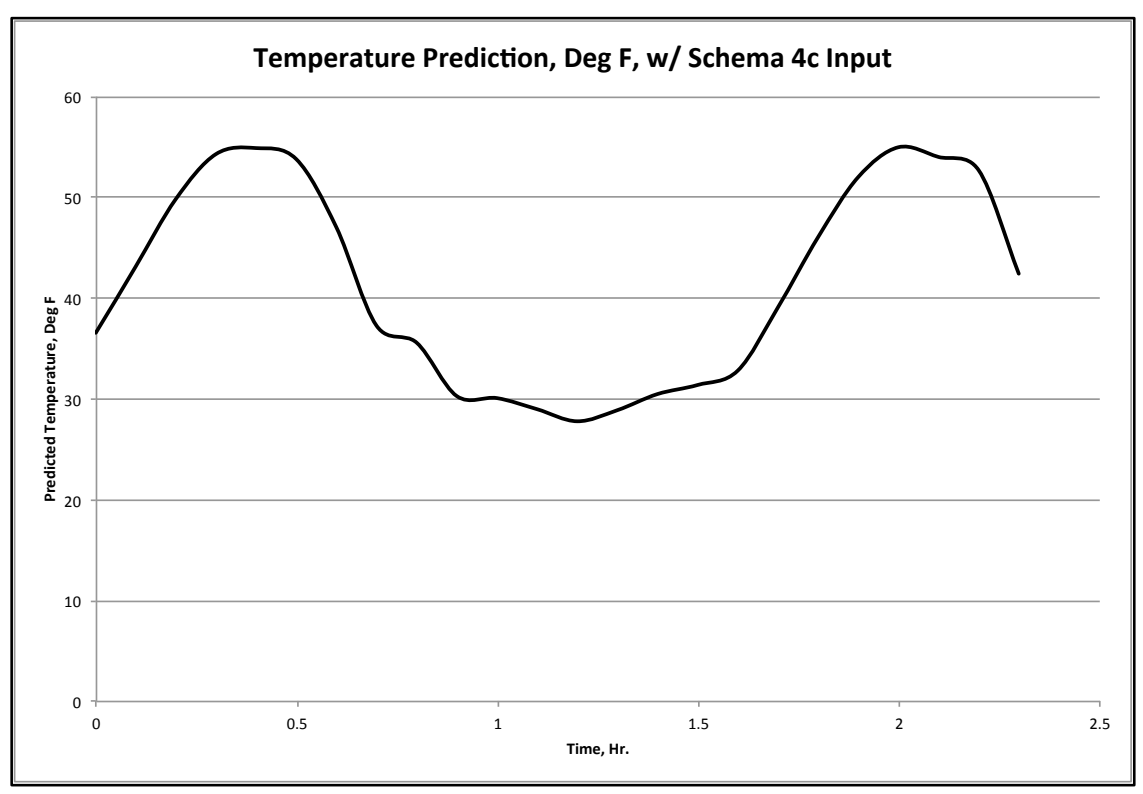

Figure 109. SINDA Output w/ ADC On \& 60s (Schema 4c) 


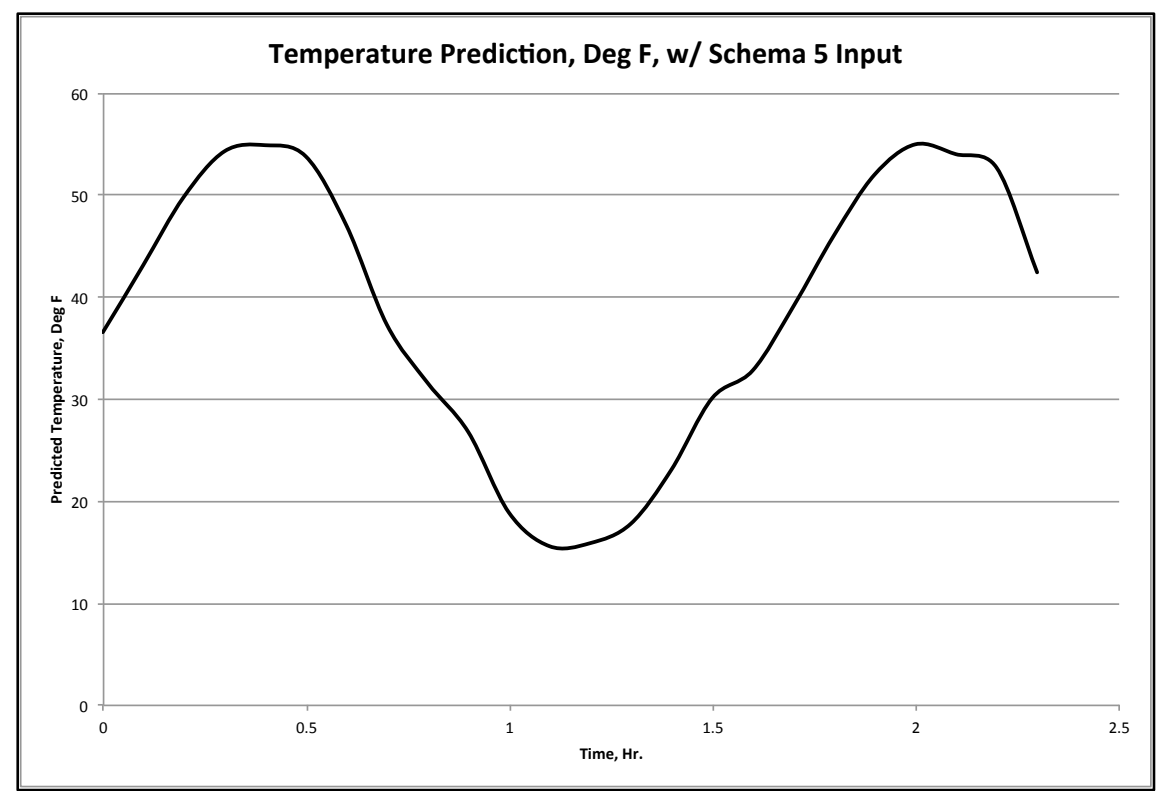

Figure 110. SINDA Output w/ Increased Contact/ Data Drop (Schema 5) 


\section{APPENDIX K. SMC THERMAL GRADIENT FAILURE RATES}

To provide background the following information was gathered that compares the fatigue, cracking and issues caused by thermal cycling on surface mount components. The following figures and data are from a testing performed by Takayuki Nagai at Japan's Espec Test Center Corporation. ${ }^{32}$

\begin{tabular}{|c|c|c|c|}
\hline \% & $\begin{array}{l}\leftarrow 1608 \\
\leftarrow 2012\end{array}$ & $\begin{array}{l}\text { Resistor } \\
(0 \Omega)\end{array}$ & $\begin{array}{l}1608(1.6 \times 0.8 \mathrm{~mm}) \\
2012(2.0 \times 1.25 \mathrm{~mm}) \\
3216(3.2 \times 1.6 \mathrm{~mm}) \\
5025(5.0 \times 2.5 \mathrm{~mm}) \\
\text { * plating: } \mathrm{Sn}\end{array}$ \\
\hline 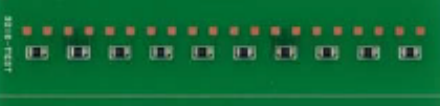 & $\leftarrow 3216$ & Solder & $\begin{array}{l}\text { Sn-37Pb } \\
\text { Sn-3.0Ag-0.5Cu }\end{array}$ \\
\hline 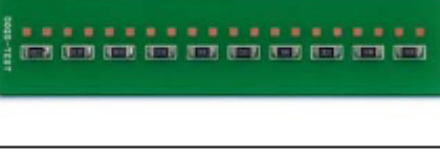 & $\leftarrow 5025$ & PCB & $\begin{array}{l}100 \times 110 \mathrm{~mm} \\
\text { thickness } 1.6 \mathrm{~mm} \\
\text { FR-4, OSP }\end{array}$ \\
\hline
\end{tabular}

Figure 111. РСВ Article for Surface Mount Component Failure Rate Test ${ }^{32}$

The surface mount components shown in figure 111 above and used for the test are identical to those used in a typical CubeSat including 1608, 2012 and occasionally 3216 resistors. In the figure below you can clearly see the increased void distance, cracking and strain being induced by the large thermal ramp or gradient rates. 


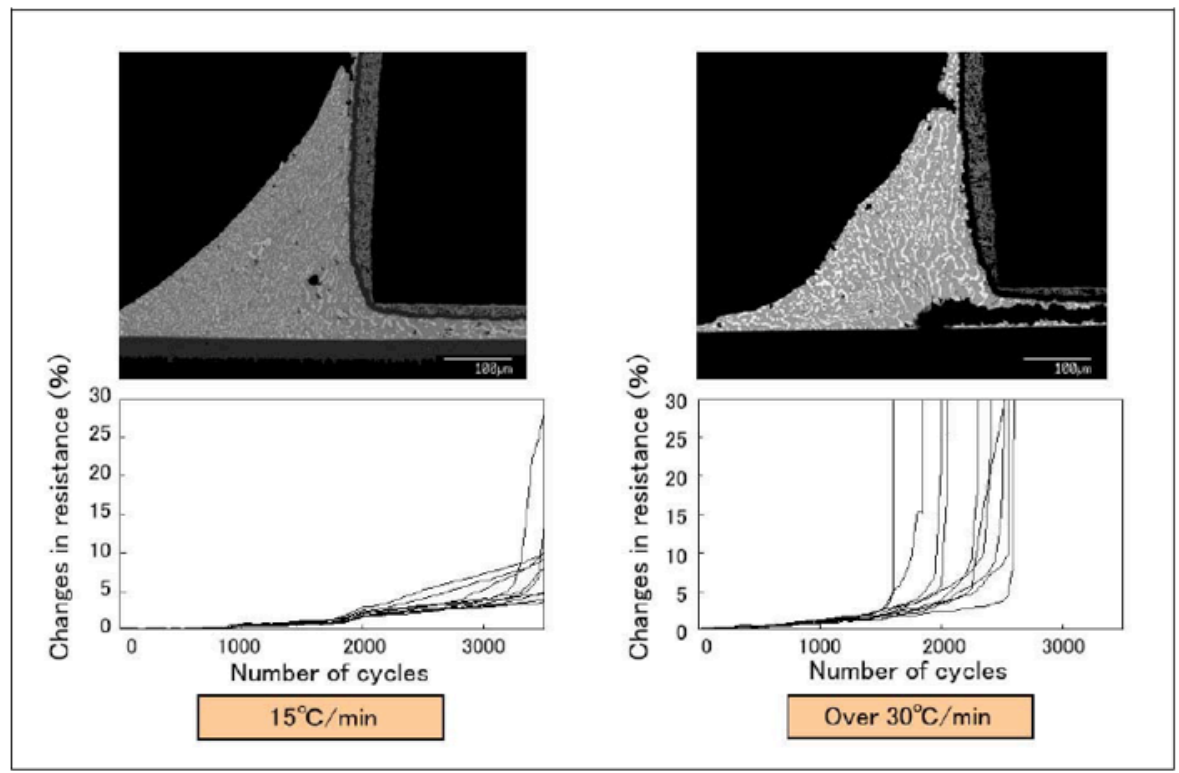

Figure 112. Solder Joint Cracking due to Thermal Cycling \& Alteration in Resistance ${ }^{36}$

In Figure 131 below you can see the higher ramp rate clearly affecting the overall solder joint connection.

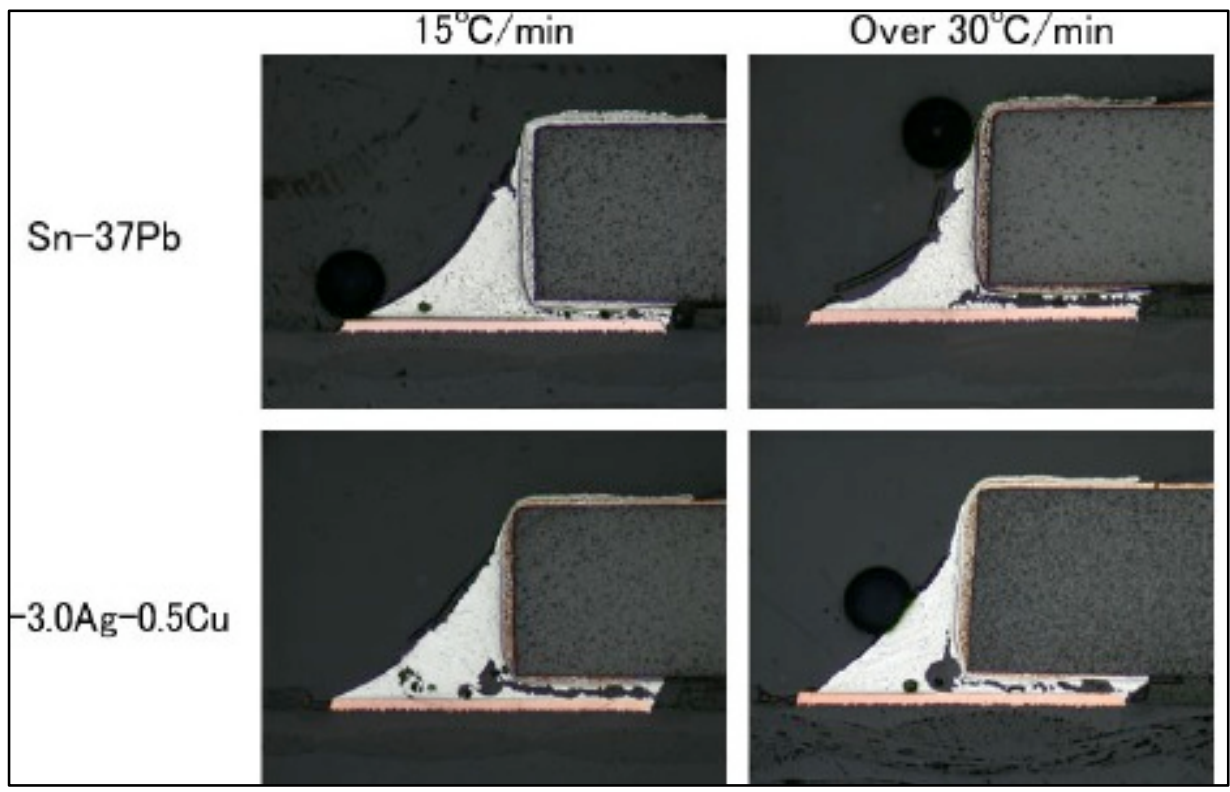

Figure 113. 1000 Cycle Thermal Test on 3216 Surface Mount Resistor 
The last figure presented illustrates the drastic reduction in cycles to failure caused by just a $15^{\circ} \mathrm{C}$ higher ramp. This same mechanism holds true during onorbit gradients and is part of the reason for maintaining as steady of a system temperature as possible will have a lifespan effect on the surface mount components that make up the majority CubeSat electronics.

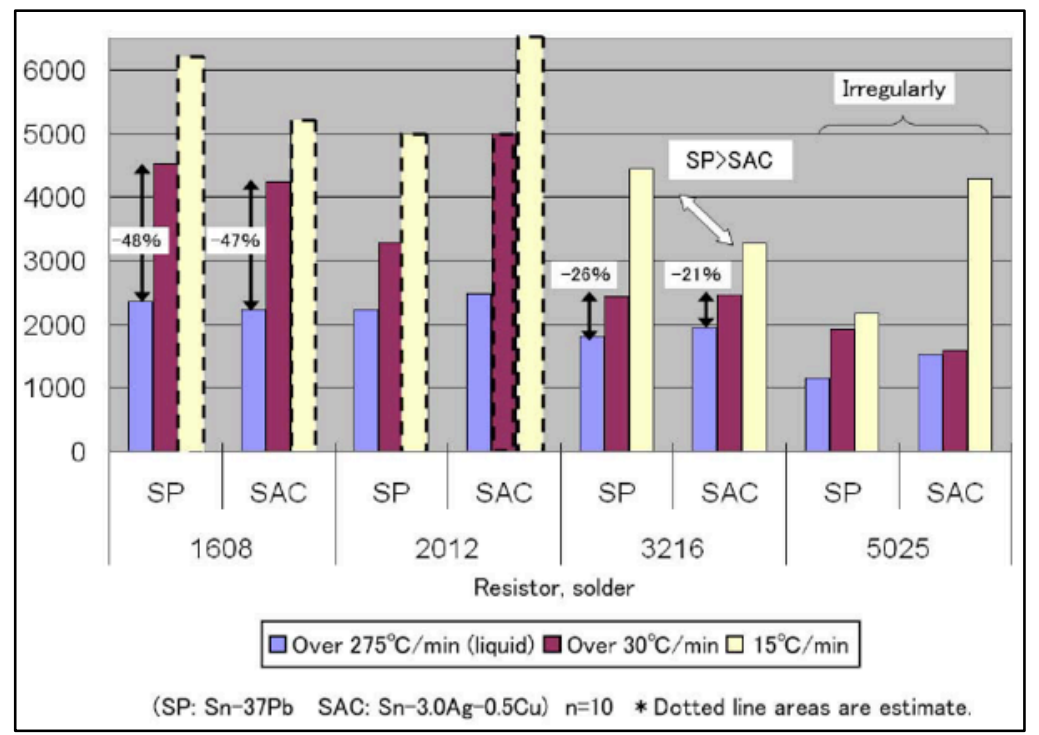

Figure 114. Failure by Resistor Type and Cycle Number for Different Ramp Gradients ${ }^{32}$ 


\section{APPENDIX L. RSSI - Test Procedure \& Results}

\section{Testing CP2 \& 3 RSSI Temperature Effects}

\begin{tabular}{|c|c|c|c|}
\hline $\begin{array}{c}\text { Revision } \\
\text { Number }\end{array}$ & Changes Made & Changes Made By & $\underline{\text { Date Changes }}$ \\
\hline 0 & Document Creation & William Whalen & May 28, 2007 \\
\hline 1 & Alteration for 2 ${ }^{\text {nd }}$ Test & William Whalen & June 4, 2007 \\
\hline
\end{tabular}

Antenna Positions:

Marconi:

Elevation: 0 degrees

Azimuth: 60 degrees

Hertz:

Elevation: 0 degrees

Azimuth: 60 degrees 


\section{Table of Contents}

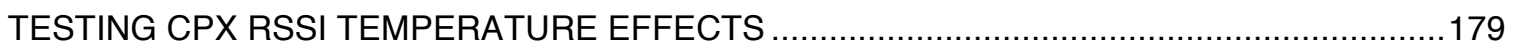

1.

PURPOSE

1.1 Primary Objective 129

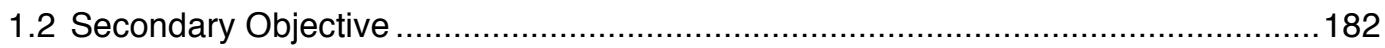

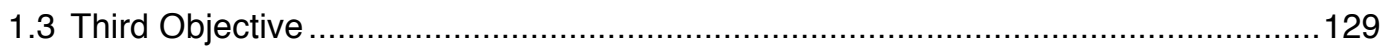

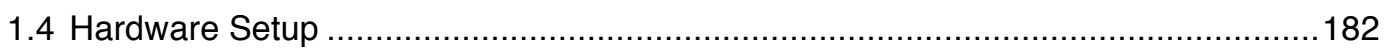

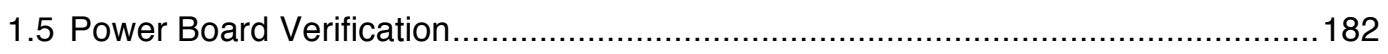

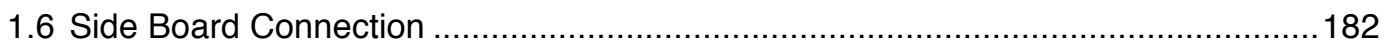

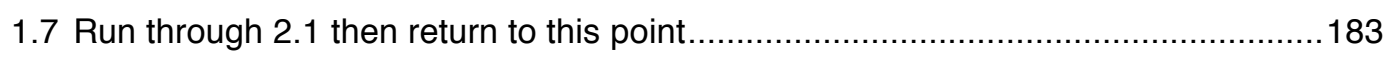

1.8 Prepare TestSat for moving to T-Vac location ................................................ 183

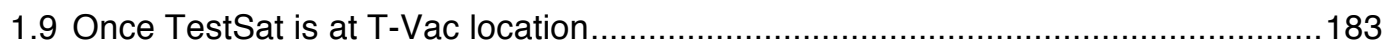

2. TEST CONDITIONS 183

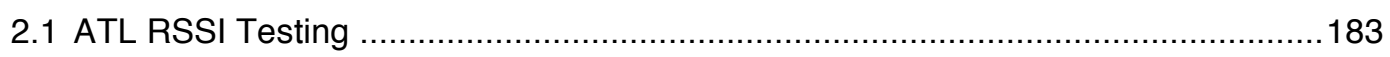

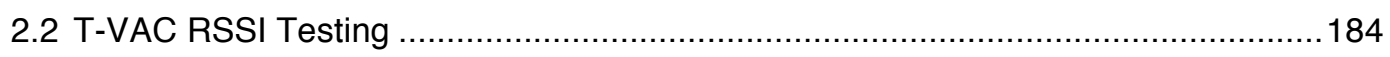

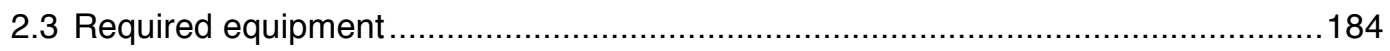

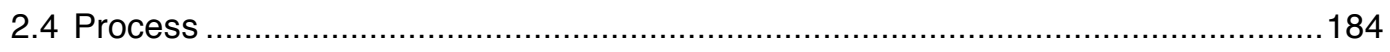

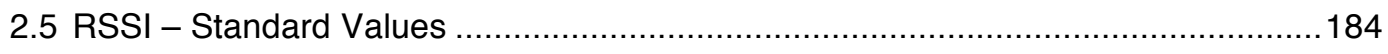

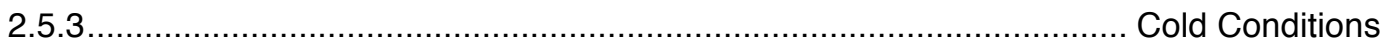

185

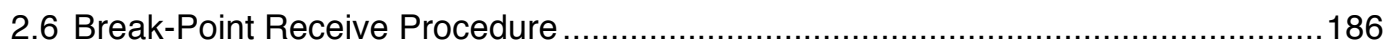

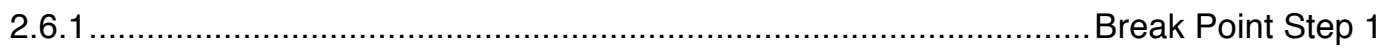


2.6.2. Break Point Step 2

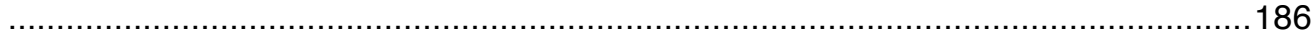

2.6.3 186

3. DATA ANALYSIS 186

3.1 Determining the relationship ... 186 
Purpose

\section{Primary Objective}

The primary purpose of this experiment is to find the relationship between RSSI readings and the temperature in which the CP3 and CP2 assembly has been placed. We will stress the objective of placing the boards in its on-orbit situations.

\section{Secondary Objective}

The secondary purpose of this experiment is to test magnetometer readings fluctuations with temperature. Previous tests only ran the magnetometers to $-10 \mathrm{C}$ at which point some erroneous readings were found. This test will take the magnetometers down to actual on-orbit temperatures of -35 to $-40 \mathrm{C}$.

\section{Third Objective}

The last objective of this experiment is, if possible, to quantify heating values with operational commands and operations. This will require data analysis post-test.

\section{Hardware Setup}

1.1.1 Prepare TestSat for Thermal Vacuum chamber.

1.1.2 Check for any loose connections between boards

1.1.3 Prepare ground station for downloading data during test

1.1.3.1 Create "T-VAC Test" Folder on Hertz and Marconi

1.1.3.2 Make directory for Parsed file saving as aforementioned folder

\section{Power Board Verification}

1.1.4 Turn the multimeter on to the voltmeter mode.

1.1.5 Use the banana-to-banana connectors to connect the multimeter to the "V Batt" and "Batt Gnd" ports on the umbilical box.

1.1.6 If the multimeter reads a voltage less than $3.8 \mathrm{~V}$, replace or charge the Power board and repeat steps 1.1 .4 to 1.1 .6 until the voltage is $3.8 \mathrm{~V}$ or greater.

1.1.7 Disconnect multimeter.

\section{Side Board Connection}


1.1.8 Put on gloves.

1.1.9 Put on a grounding strap and snap into a ground.

1.1.10 Connect the 10-pin cable coming out of JP7 on the power board into JP1 on the side board.

1.1.11 This allows for sideboard readings to be taken

Run through 2.1 then return to this point

Prepare TestSat for moving to T-Vac location

Once TestSat is at T-Vac location

1.1.12 Check RSSI 5 times outside chamber

$$
\text { 1.1.12.1.1 }
$$

1.1.12.1.2

1.1.12.1.3

1.1.12.1.4

1.1.12.1.5

1.1.12.1.6

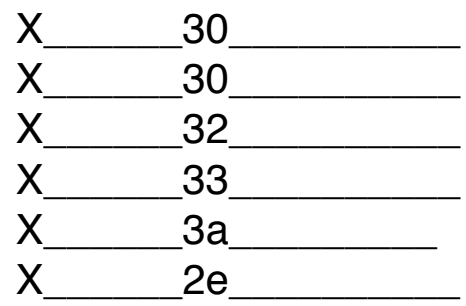

1.1.13 Check RSSI 5 times inside chamber
1.1.13.1.1
1.1.13.1.2
1.1.13.1.3
1.1.13.1.4
1.1.13.1.5

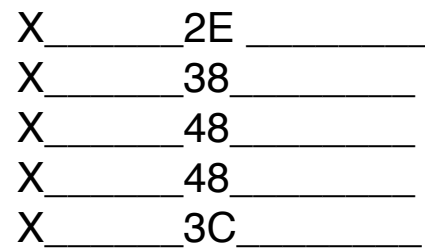

1.1.14 Prepare to T-Vac - go to section 2.2

\section{Test Conditions}

\section{ATL RSSI Testing}

1.1.15 Record the name of the test satellite being used.

1.1.16 While inside ATL lab, follow procedure 1.4.

1.1.16.1 Record power level.

1.1.16.2 Point the antennas towards the T-Vac chamber

0 degrees elevation is still approximately 3 (speculate that the reason is because the weight of the coaxial cable is pulling it down). 1.1.17 While still inside ATL, remove RBF pin and using interior lab antenna, check to be sure TestSat is beaconing.

Testsat is beaconing.

1.1.18 Change beacon rate from 1 every 5 minutes to 1 every 2 minutes 1.1.18.1 Record 5 RSSI level readings inside lab.
1.1.18.1.1
$\mathrm{X}$ 0 


$\begin{array}{ll}1.1 .18 .1 .2 & \mathrm{X} \\ 1.1 .18 .1 .3 & \mathrm{X} \\ 1.1 .18 .1 .4 & \mathrm{X} \\ 1.1 .18 .1 .5 & \mathrm{X} \\ 1.1 .18 .1 .6 & \mathrm{X} \\ 1.1 .18 .1 .7 & \mathrm{X} \\ 1.1 .18 .1 .8 & \mathrm{X} \\ 1.1 .18 .1 .9 & \mathrm{X} \\ 1.1 .18 .1 .10 & \mathrm{X}\end{array}$

1.1.19 Change beacon rate from 1 every minute.

1.1.19.1 Record 5 RSSI level readings from inside lab - make sure all beacons are decoded

$\begin{array}{lll}1.1 .19 .1 .1 & \mathrm{X} & \mathrm{OF} \\ 1.1 .19 .1 .2 & \mathrm{X} & \mathrm{OF}- \\ 1.1 .19 .1 .3 & \mathrm{X} & \mathrm{OF}- \\ 1.1 .19 .1 .4 & \mathrm{X} & \mathrm{OF} \\ 1.1 .19 .1 .5 & \mathrm{X} & \mathrm{OE}\end{array}$

\section{T-VAC RSSI Testing}

\section{Required equipment}

1.1.20 CDH/power board sandwich

1.1.21 Side boards

1.1.22 Structure-Integrated

1.1.23 Kapton tape

1.1.24 Ribbon cable and extensions

\section{Process}

1.1.25 Set up equipment in the lab to ensure all the components are working before going to the thermal vac chamber - per instruction in section 1 \& 2

1.1.26 Place the TestSat on an isothermal \& isolation stand

\section{RSSI - Standard Values}

1.1.27 Record initial mag field and temperature readings 
1.1.28 Cycle the board thru the determined temperature range.

\subsubsection{Cold Conditions}

Using the thermal vacuum chamber, the satellite's external temperatures will be brought down to $-36 \mathrm{C}$, internally it will reach $-20 \mathrm{C}$, the following levels will be checked for RSSI and Magnetometer characterization.

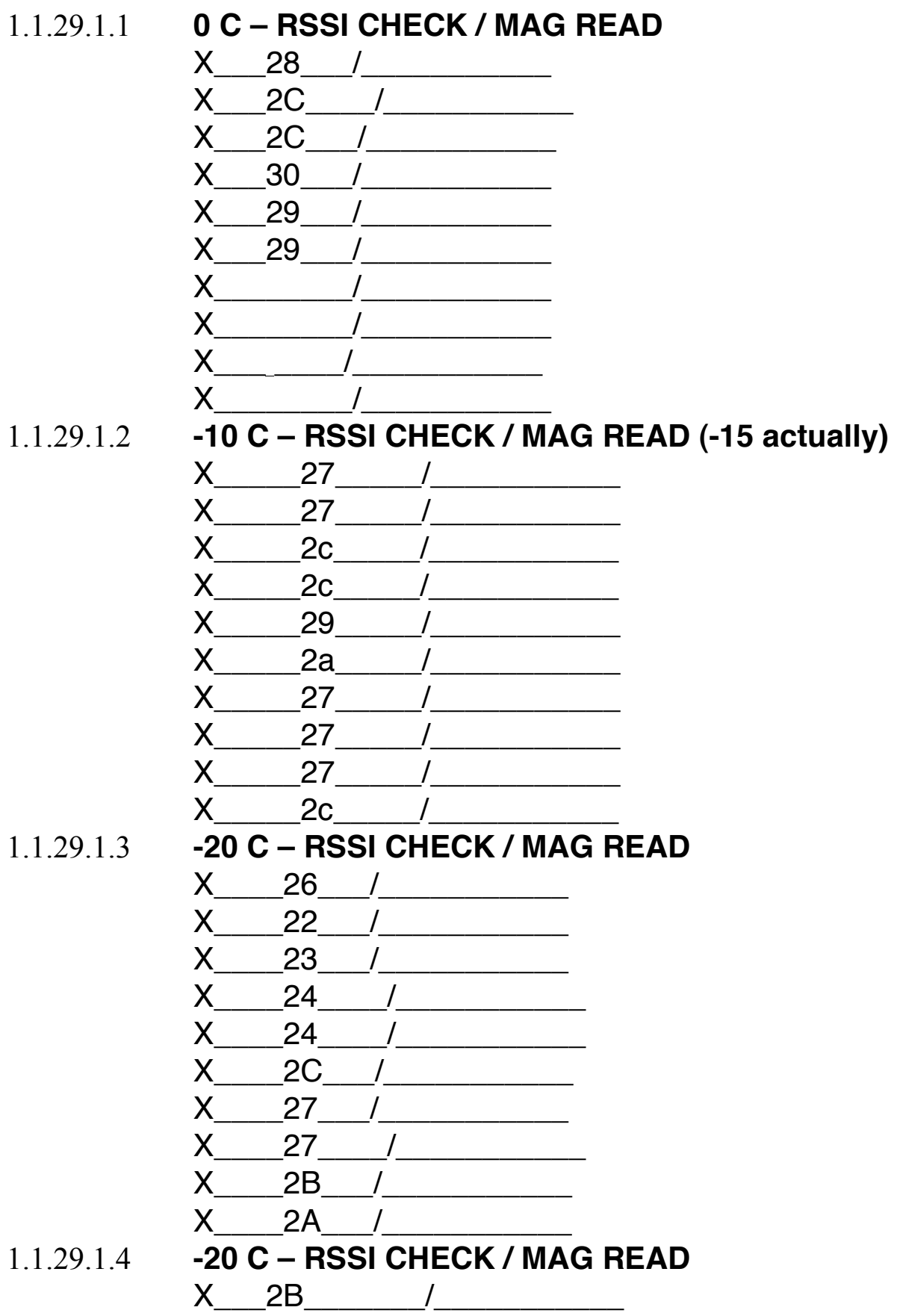




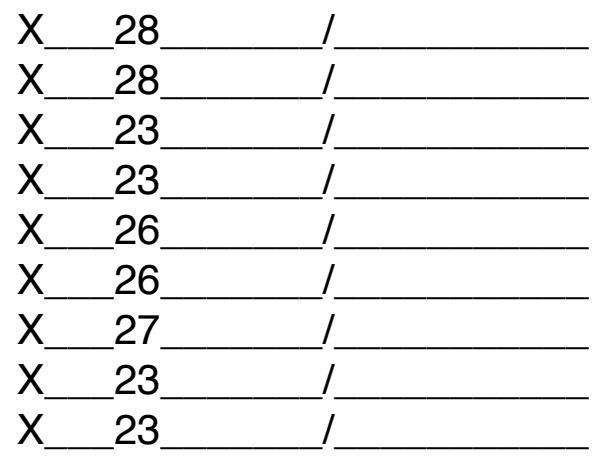

\section{Break-Point Receive Procedure}

If Satellite is still receiving signal from ground station at $-20 \mathrm{C}$ perform the

following two steps.

\section{Break Point Step 1}

Lower RF gain on groundstation until RSSI is at "break point" (HEX Recieved / 255 decimal ${ }^{*} 3$ volts $=$ RSSI)

\section{Break Point Step 2}

As satellite warms monitor RSSI readings from 30 second snapshots.

\section{Hot Conditions - NONE}

\section{Data Analysis}

\section{Determining the relationship}

1.1.30 Plot RSSI values on the $y$-axis against temperature on the $\mathrm{x}$-axis 1.1.31 Plot cut-off points 
1.1.32 Plot sensor dump information

1.1.33 Plot Temperature on the $x$-axis and mag readings on the $y$-axis.

1.1.34 Plot using points only and then add a best-fit line to find the relationship on all graphs

\section{Additional Notes:}

$1^{\text {st }}$ Test -Breakpoint was found to be one deg (mark) less than half RF power

$2^{\text {nd }}$ Test- Breakpoint was found to be four deg (mark) less than half RF power

Plots done using data point \# and not by interval \#.

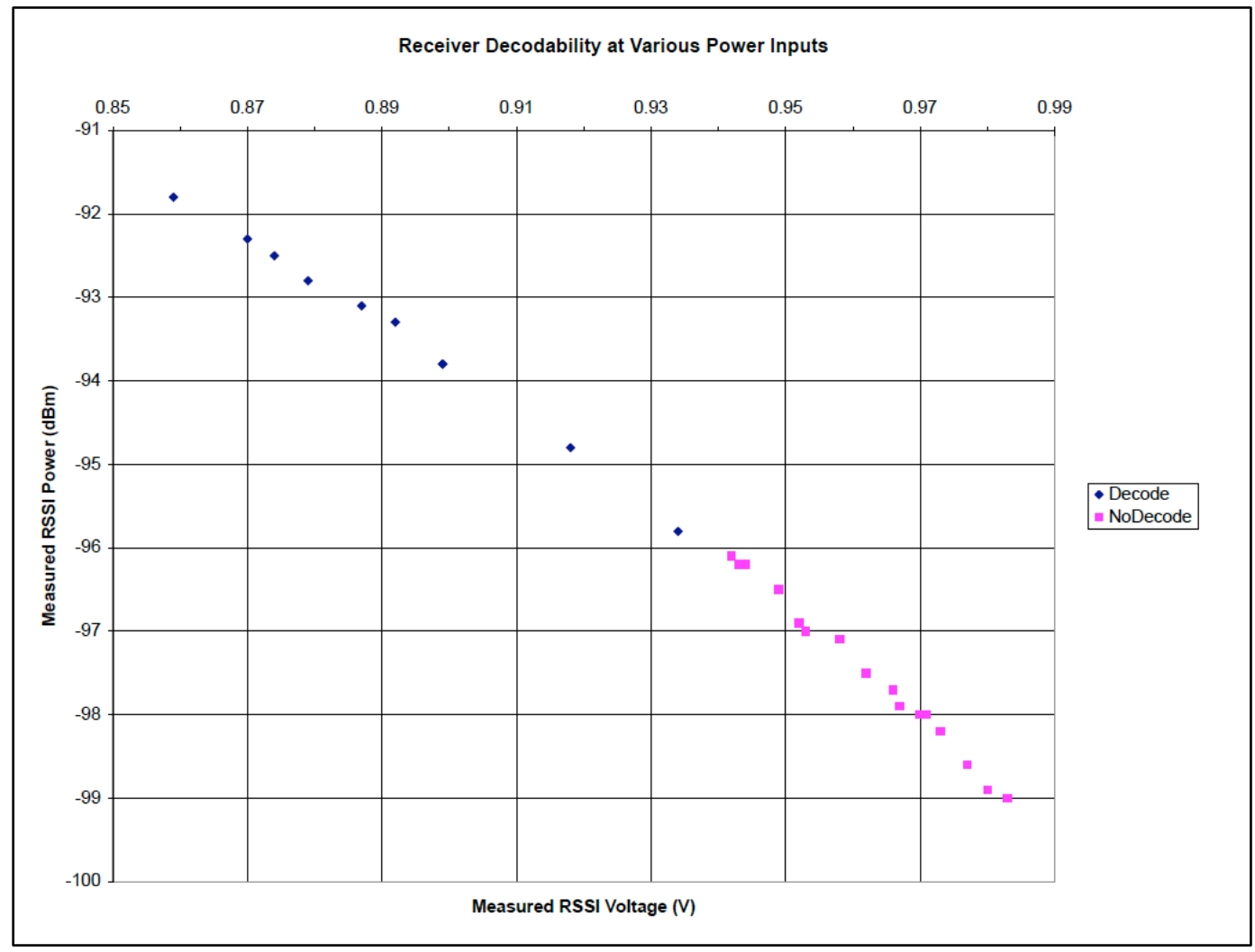

Figure 115. RSSI Receive Sensitivity vs. Power Inputs (Use for Comparison) 


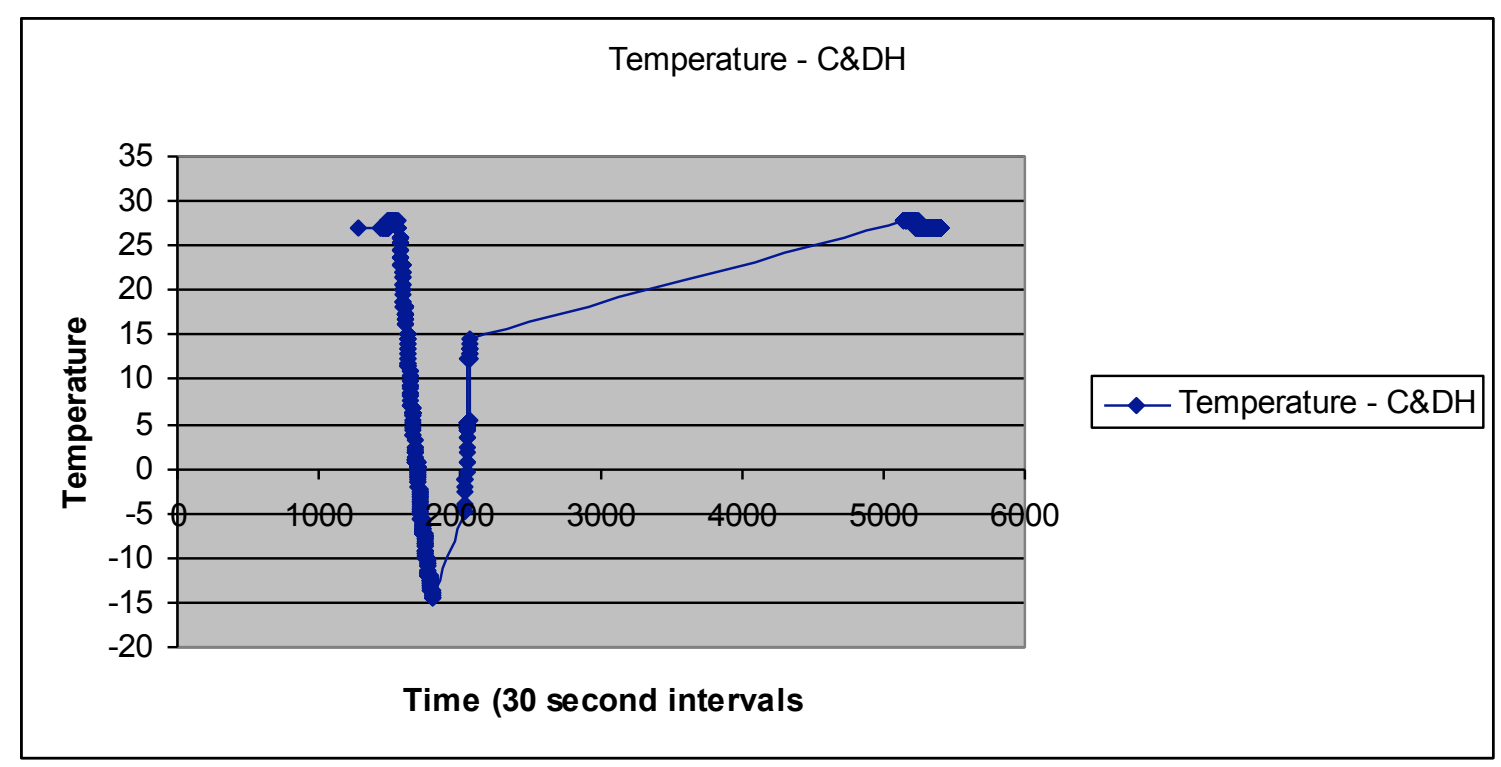

Figure 116. C\&DH Temperature During Cycle \#1

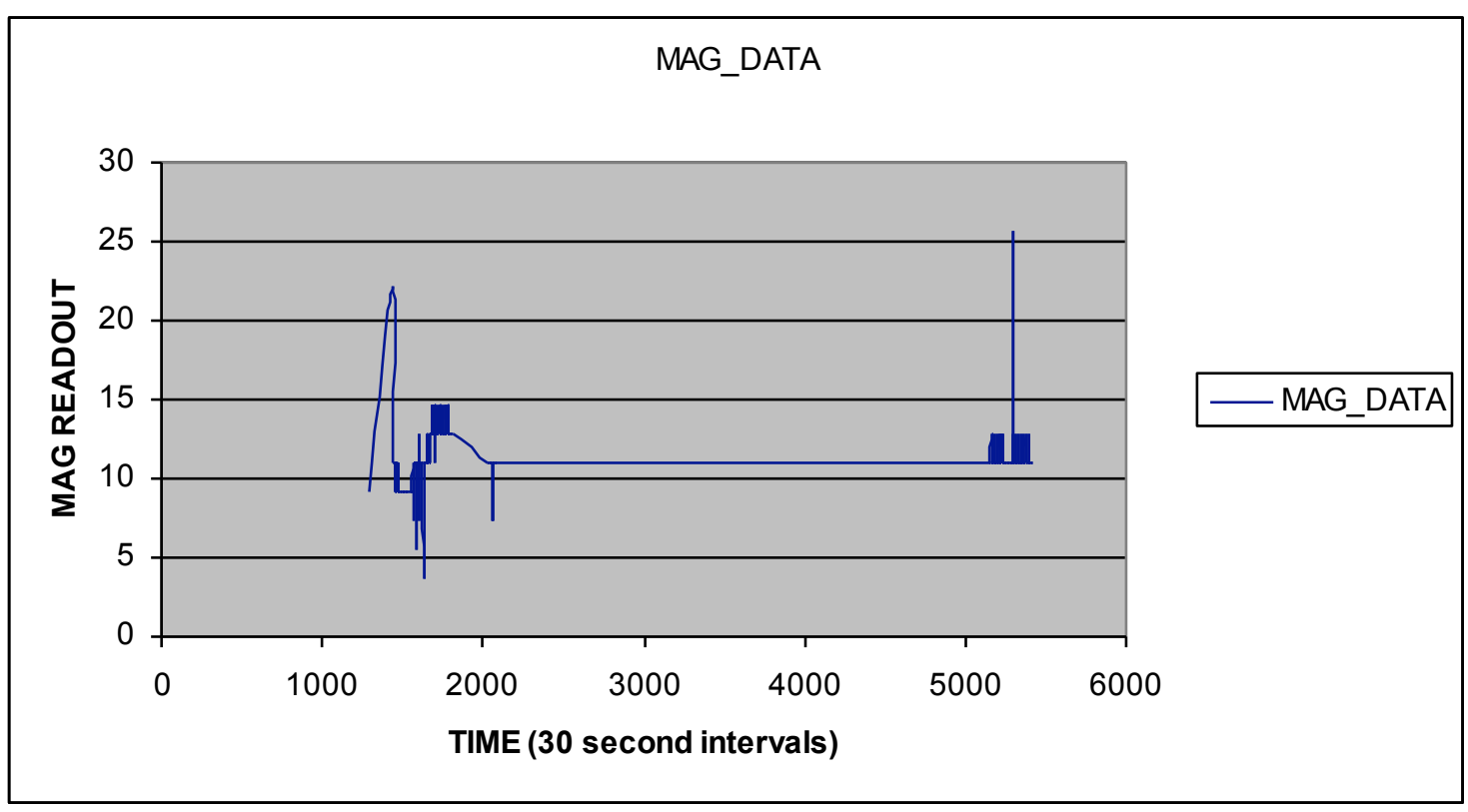

Figure 117. MAG Data Readings During Cycle \#1 


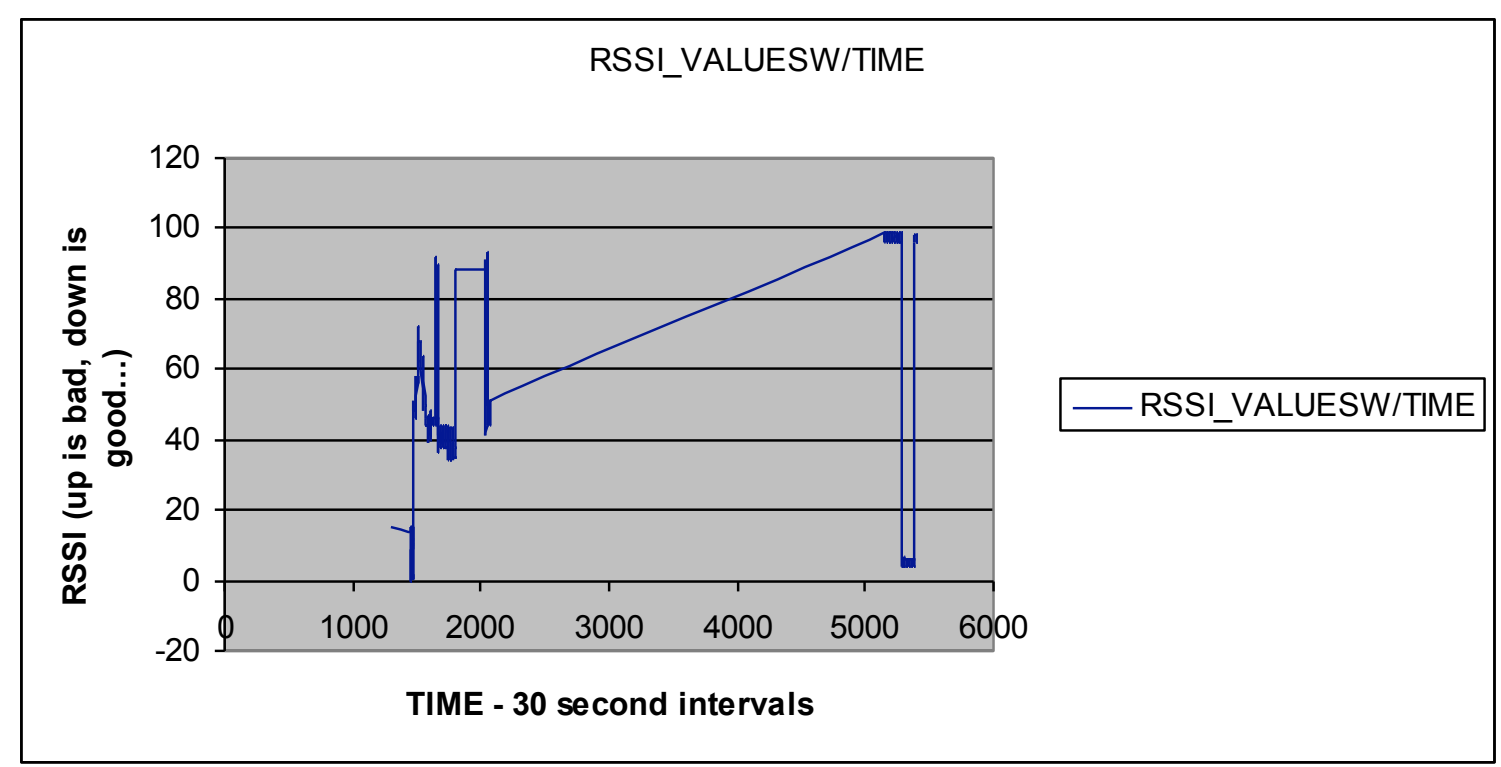

Figure 118. RSSI Values For Cycle \#1 


\section{APPENDIX M. COMPONENT TEMPERATURE SENSITIVITIES}

The following figures all relate to the effect of temperature on different components inside the CP3 system. These are all representative of components integrated into the majority of picosatellites.

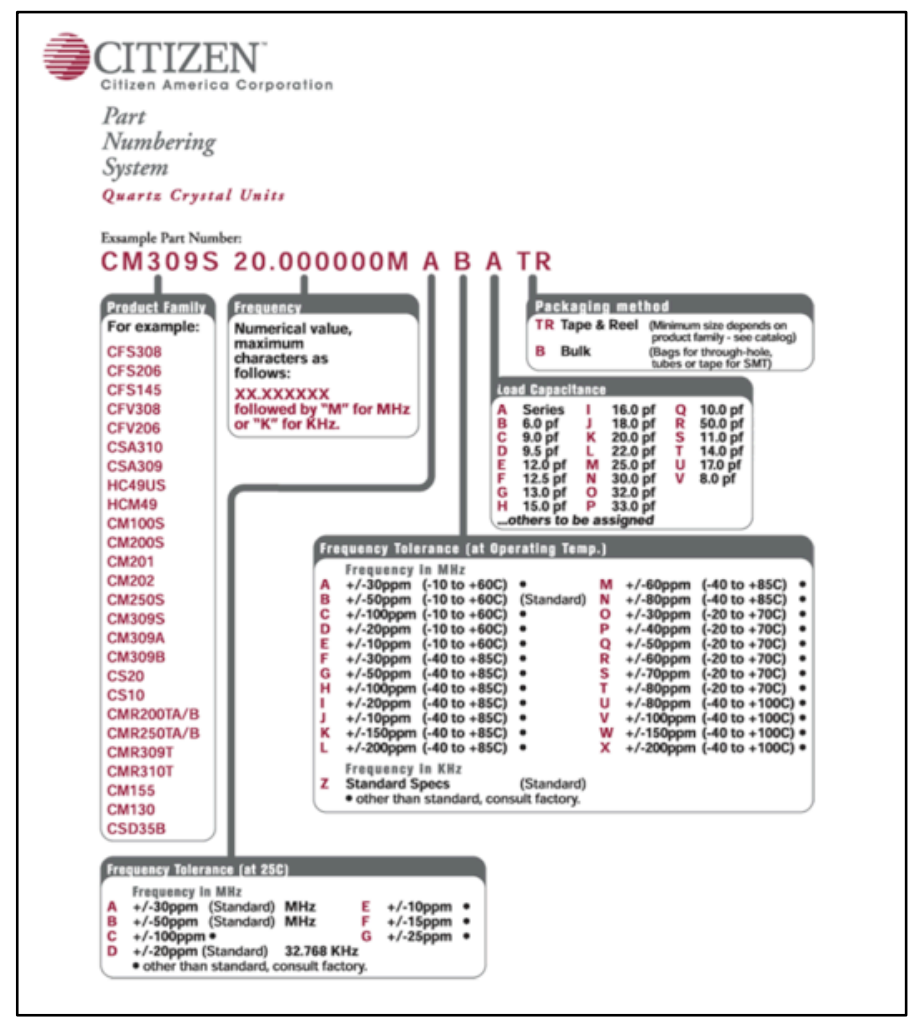

Figure 119. Citizen Crystal Oscillator Variances ${ }^{33}$

The crystals used for timing on both the $\mathrm{CC} 1000$ transceivers and the $\mathrm{C} \& \mathrm{DH}$ processors have a strong temperature dependence. As can be seen in figure 119 above and figure 120 below the HCM49's have a +/-25 ppm frequency value at normal operating temperatures which increases to $+/-50 \mathrm{ppm}$ when off the standard temperature $\left(25^{\circ} \mathrm{C}\right)$. 


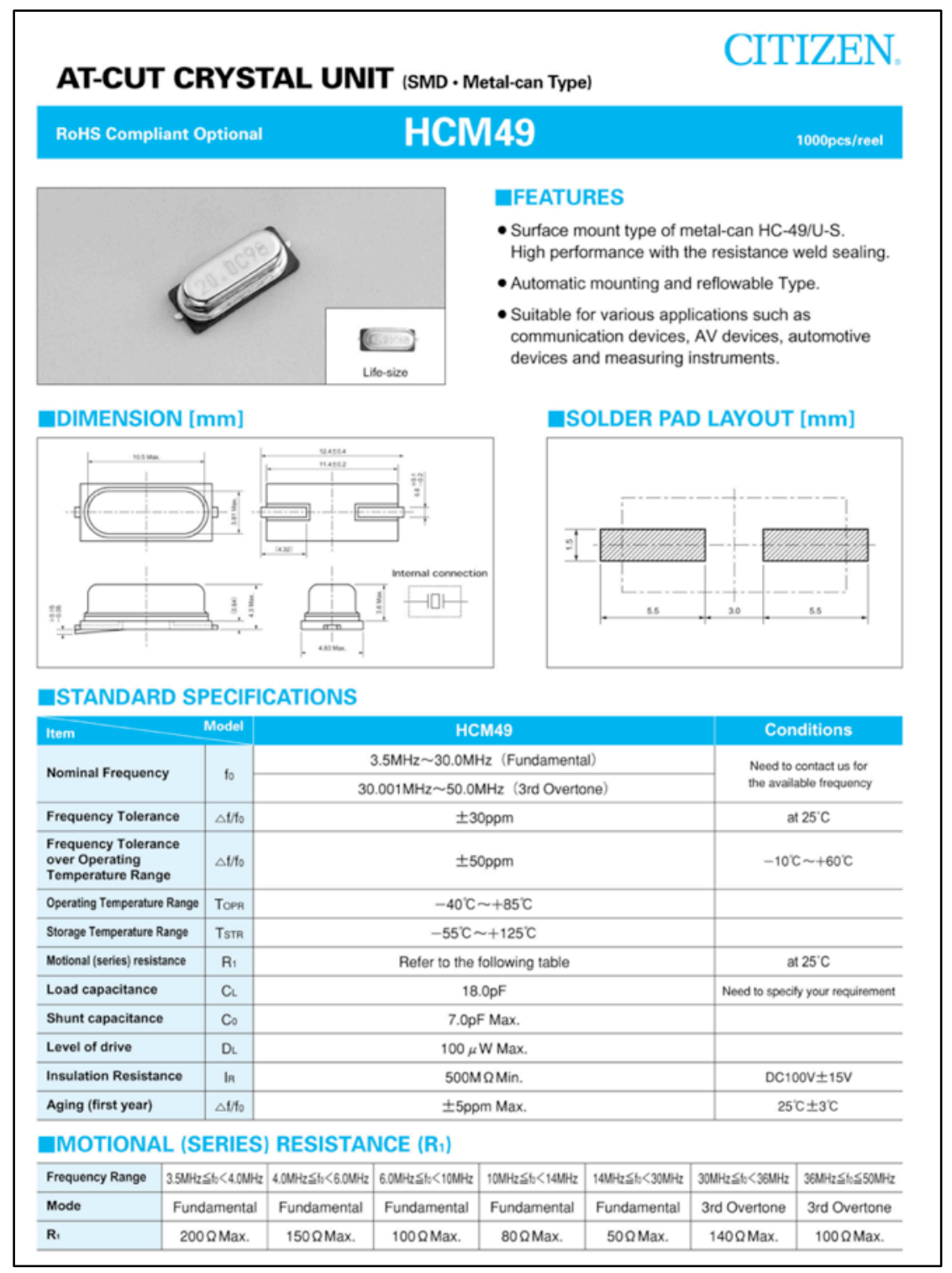

Figure 120. CC1000 and C\&DH Reference Oscillator - Citizen HCM49 ${ }^{33}$

For our CubeSat operating at approximately $400 \mathrm{MHz}$ the normal clock frequency shift would equate to a window of around $10.4 \mathrm{KHz}$ but with the added temperature uncertainty the frequency shift increases to $21 \mathrm{Khz}$. This could not only cause bus affects for timing purposes but also issues on the communication system as well. The figure below shows an uncompensated crystal oscillator's shift over temperature. 


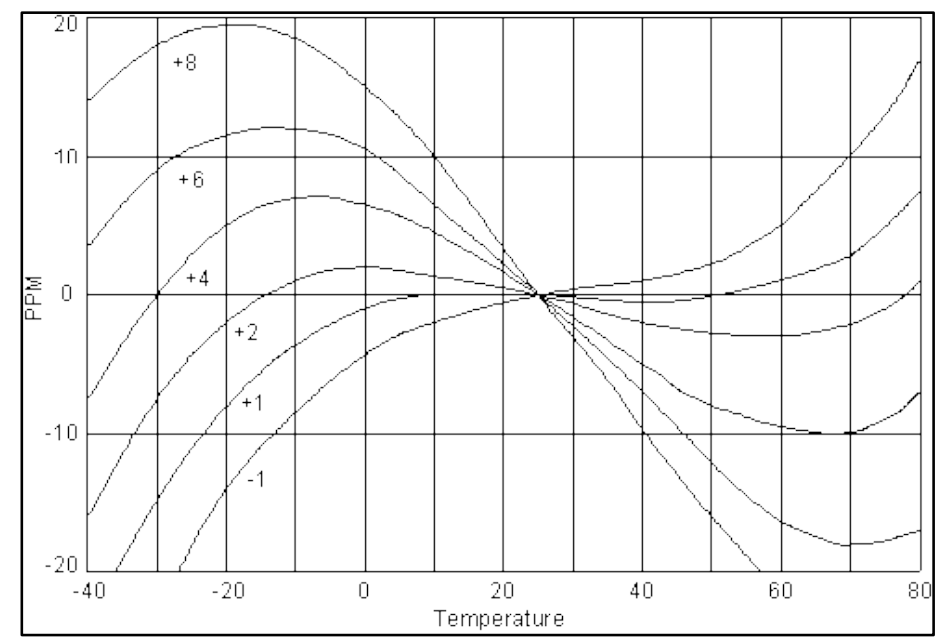

Figure 121 XO (Uncompensated) Crystal Oscillator Temperature Shift

The figure below illustrates the supply current against temperature for the DS3231 Maxim I²C real-time clock (RTC) IC.

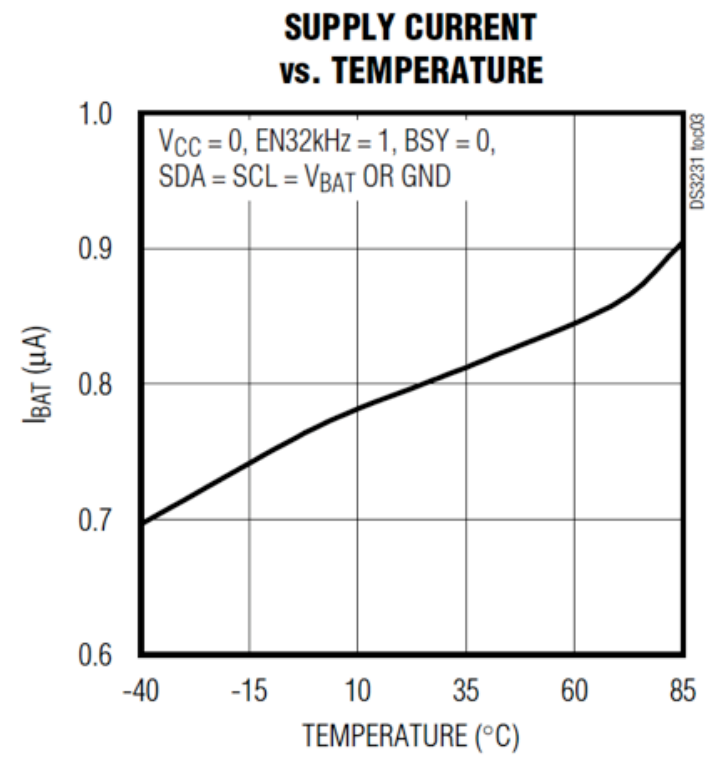

Figure 122. Supply Current vs. Temperature for the DS3231 ${ }^{34}$ 
The next figure illustrates the frequency deviation for the $\mathrm{I}^{2} \mathrm{C}$ RTC.

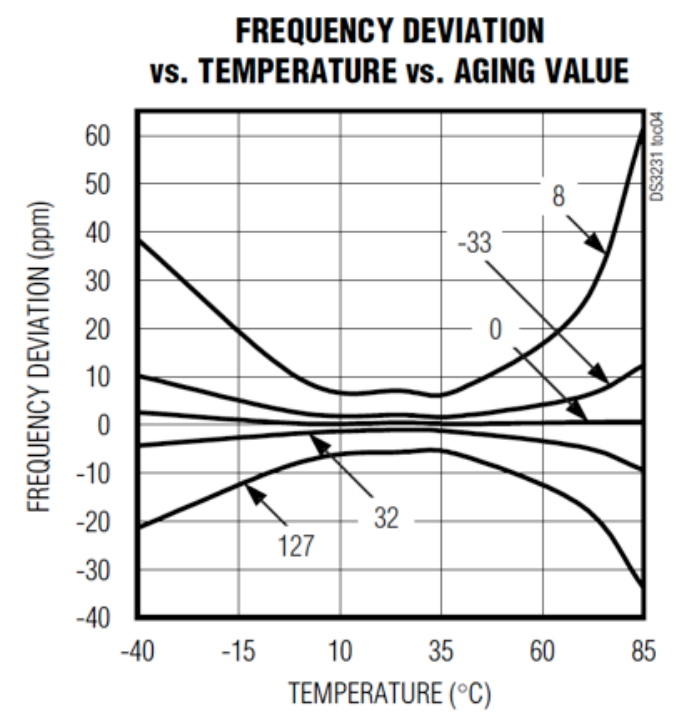

Figure 123. Frequency Deviation vs. Temperature and Aging Value for the DS3231 ${ }^{34}$

The next figure illustrates the DS3231 temperature compensated crystal oscillator (TCXO) performance against uncompensated crystal oscillators like the Citizen HCM49.

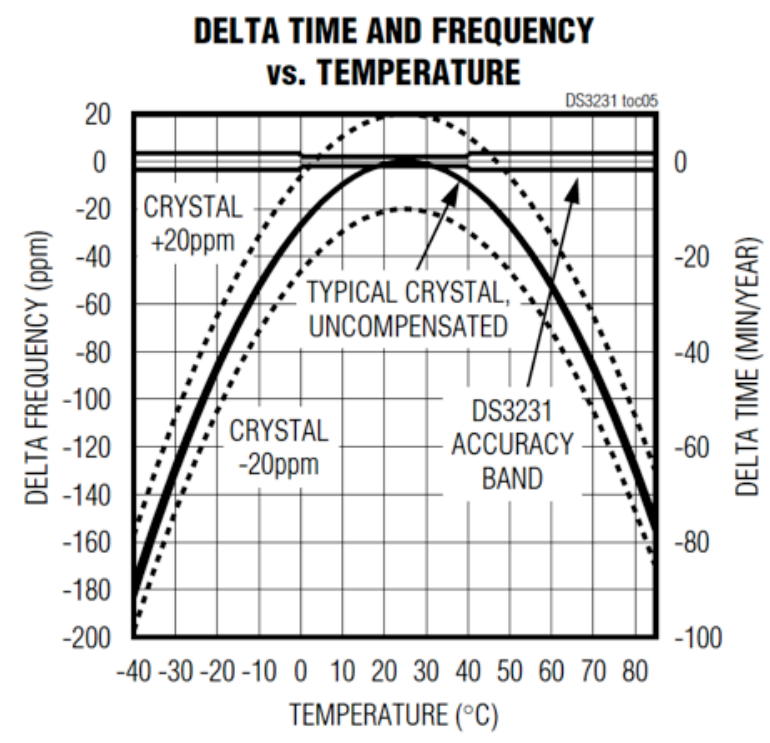


Figure 124. Delta Time and Frequency vs. Temperature for the DS3231 34

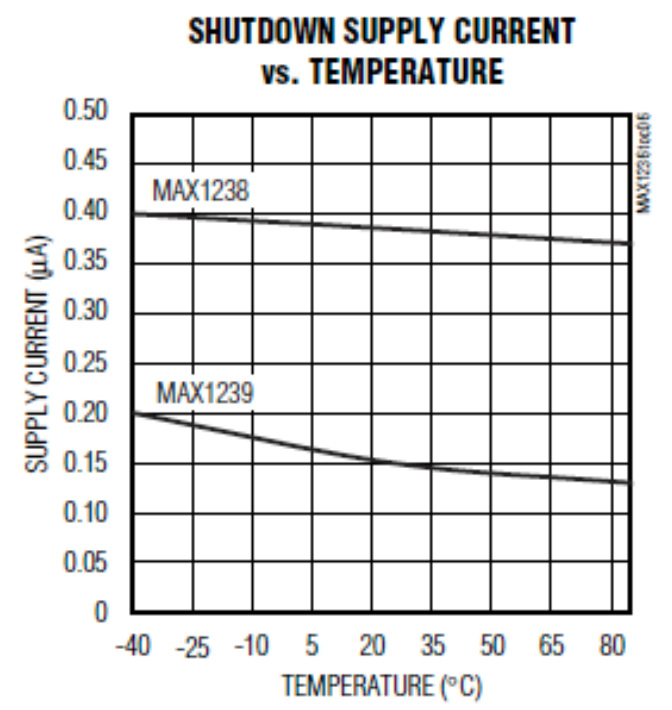

Figure 125. Shutdown Supply Current vs. Temperature for MAXIM 1239 ADCs ${ }^{12}$

Figure 123 below illustrates the drop in conductivity for the electrolyte solution inside a Lithium lon battery over temperature. This is a major contributor to the corresponding loss in specific energy shown in Figure 124.

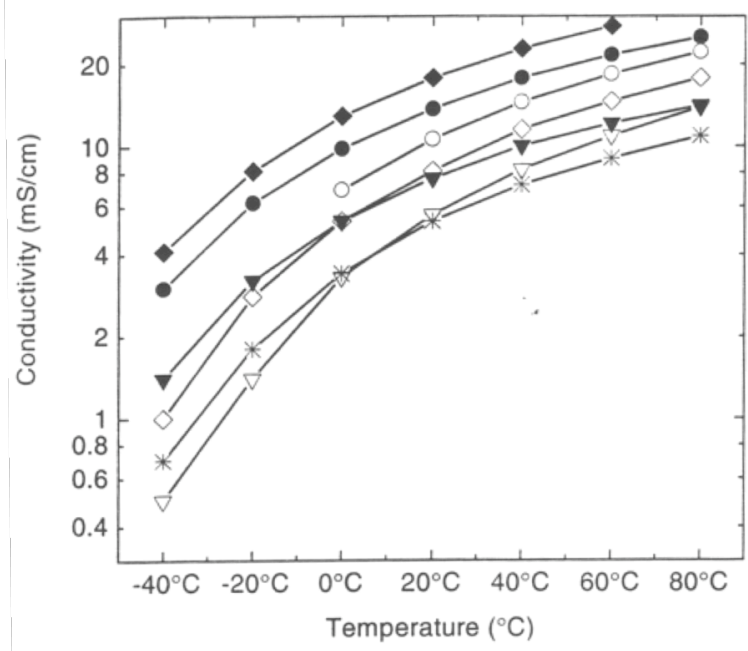

Figure 126. Lithium Ion Electrolyte Conductivity Over Temperature ${ }^{35}$ 


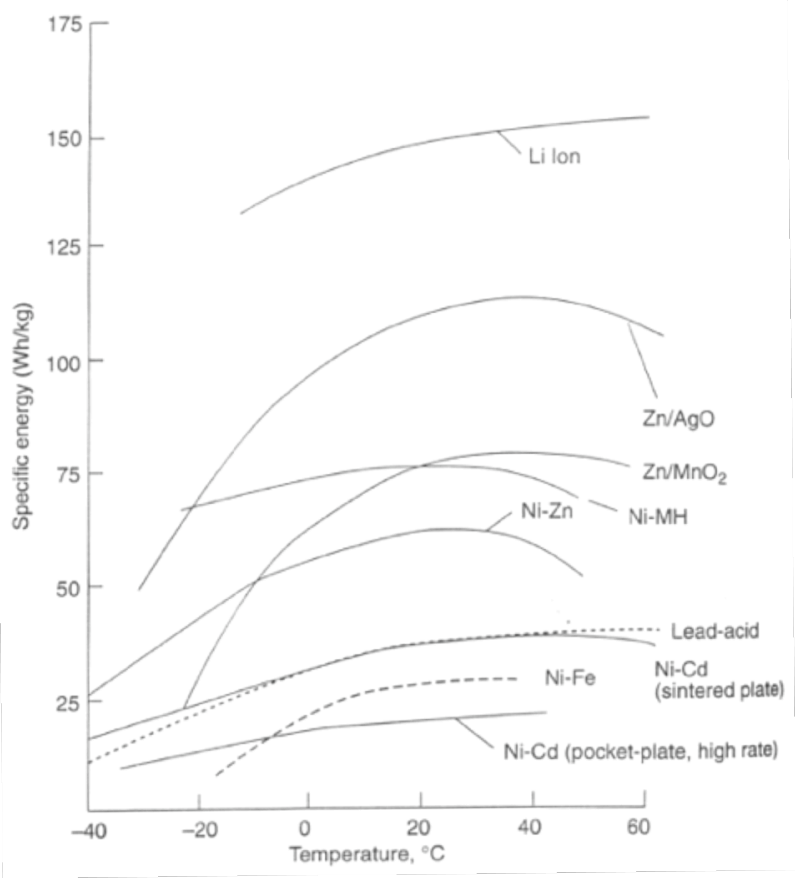

Figure 127. Specific Energy versus Temperature by Battery Type ${ }^{35}$

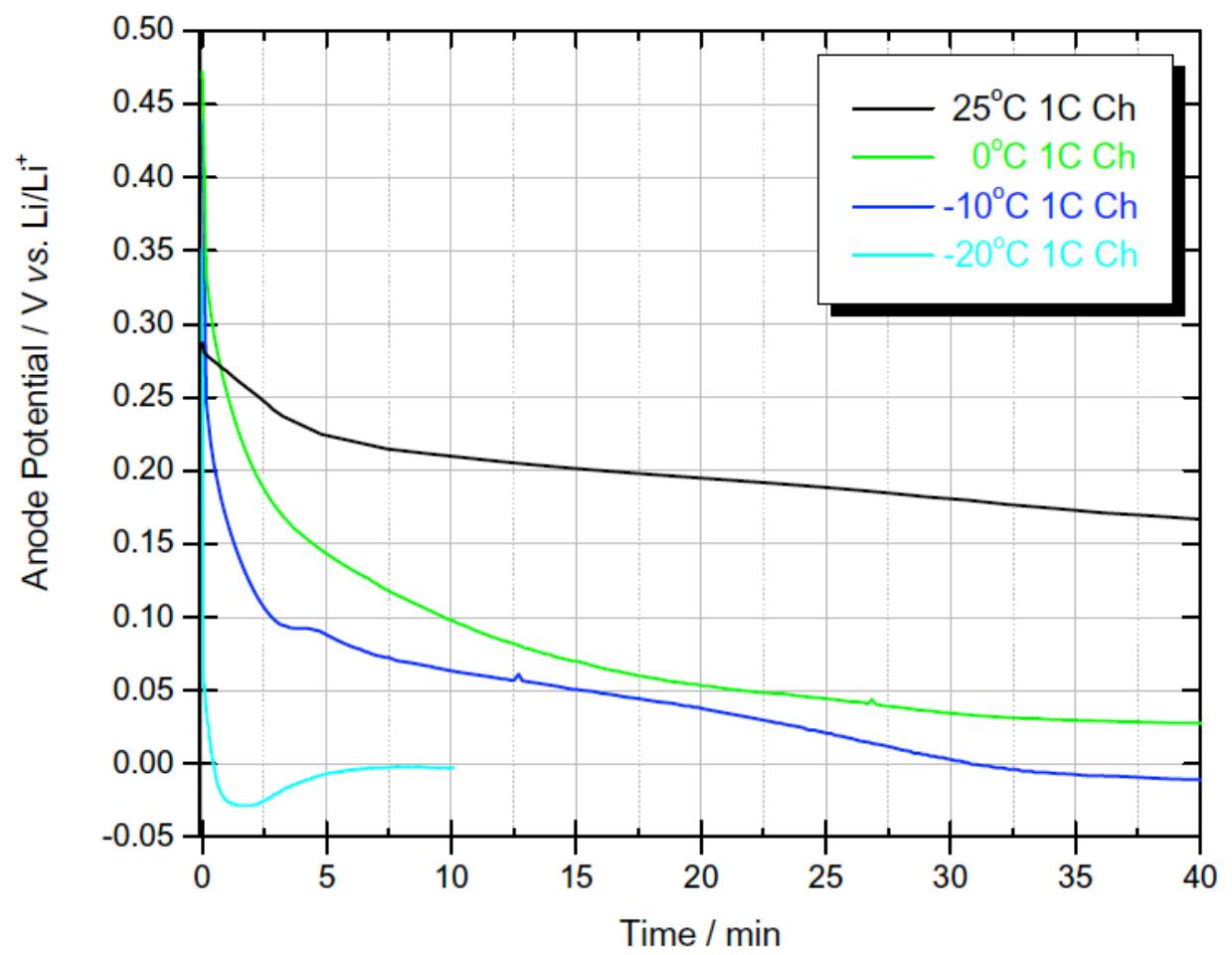

Figure 128. Lithium Ion Anode Degradation ${ }^{36}$ 
APPENDIX N. NASA THERMAL CYCLE GUIDELINES PT-TE-140230

PRACTICE NO. PT-TE-1402

PAGE 2 OF 4

\section{THERMAL CYCLING}

\section{Technical Rationale:}

Thermal cycle modeling has shown that the general form of the thermal cycling test math model is given by Equation (1).

$$
\mathrm{TE}=\mathrm{F} \times \mathrm{P}_{\mathrm{d}}\left[1-\exp \left(-\lambda_{0} \mathrm{NK}^{\Delta \mathbf{T}}\right)\right]
$$

Where: $\mathrm{TE}=$ Test Effectiveness

$\mathrm{F}=$ Fraction of total failures that can be precipitated by a thermal cycle

$P_{d}=$ Probability of detection

$\lambda_{0}=$ Failure rate at $T_{0}$

$\mathrm{N}=$ Number of thermal cycles

$\mathrm{K}=\mathrm{A}$ constant

$\Delta \mathrm{T}=\mathrm{T}-\mathrm{T}_{0}$

$\mathrm{T}=$ Operating temperature for $\lambda$

$\mathrm{T}_{0}=$ Operating temperature for $\lambda_{0}$

Fig. 1 shows that the failures available are the sum of three parts:

1. Failures detected by thermal cycle tests

2. Undetected failures

3. Failures not precipitated

For single temperature range of $50^{\circ} \mathrm{C}$, the test effectiveness equation reduces to Equation (2).

$$
\mathrm{TE}=0.9 \times \mathrm{P}_{\mathrm{d}}\left(1 \cdot \mathrm{e}^{-0.0864 \mathrm{~N}}\right)
$$

Figure 2 shows a plot of Equation (2) based on a probability of detection, $P_{d}$, of 0.9 . The equation is based on values of $\lambda_{0}$ and $K$ that were found by solving two simultaneous equations derived from the data base provided in Table 1.

Printed circuit boards (PCBs) are especially prone to solder joint cracking. The design is required to minimize the mechanical forces, as generated by thermal mismatch of materials or vibration, in the solder joints. 


\section{THERMAL CYCLING}

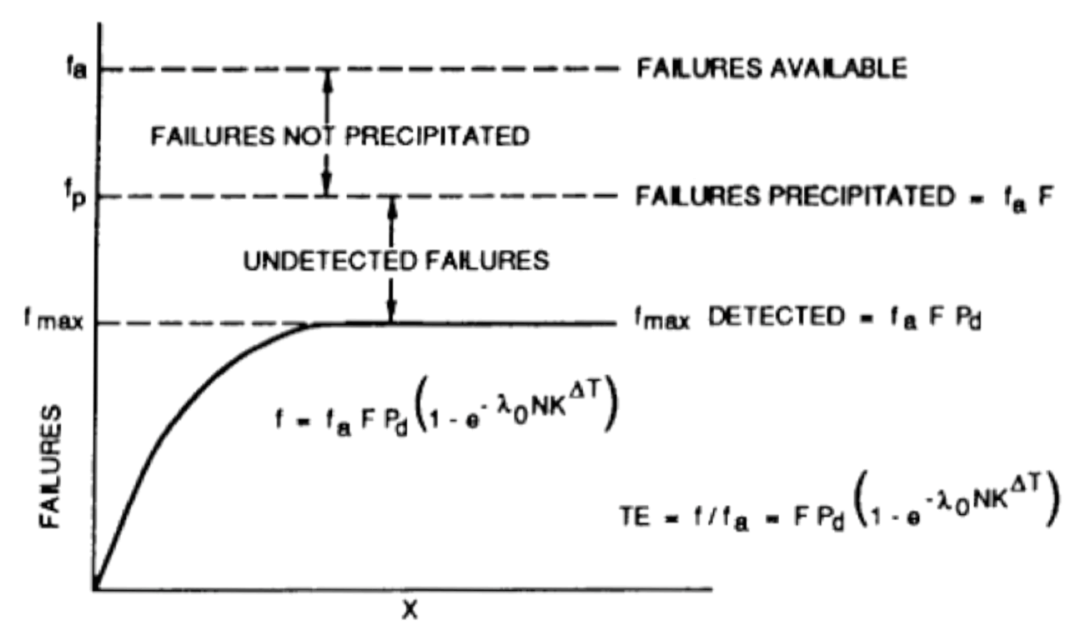

Fig. 1 General Form of TC Test Model

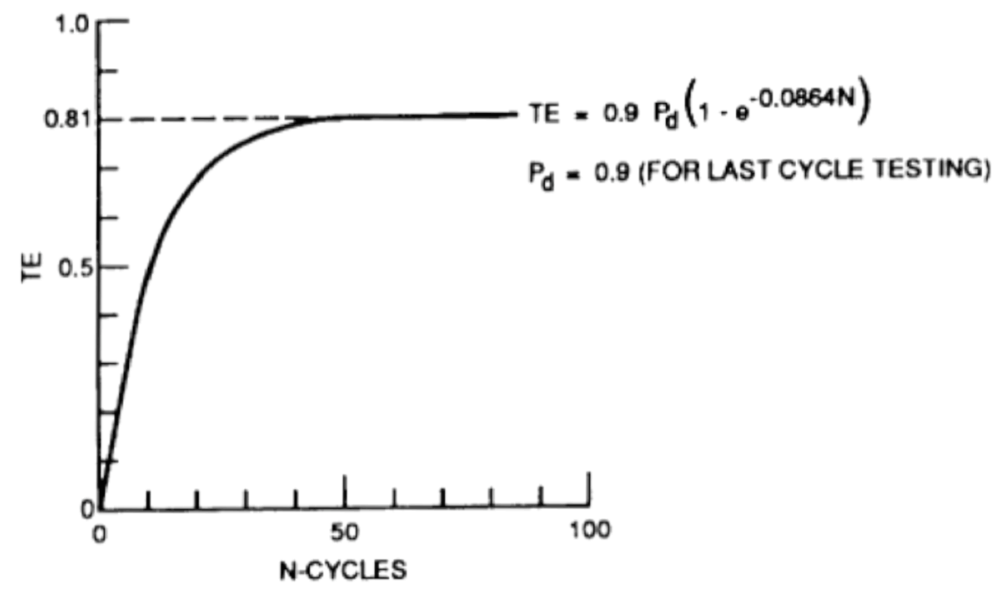

Fig. 2 Test Effectiveness Plot for $\Delta \mathrm{T}=50^{\circ} \mathrm{C}$ 


\section{Appendix O. Temperature Measurement on the CP Bus ${ }^{37}$}

This appendix is an excerpt from Chris Alan Day's Masters Thesis "The Design of an Efficient, Elegant, and Cubic Pico-Satellite Electronics System" regarding the design of the temperature monitoring system onboard the CP satellites.

\section{Temperature Measurement}

The temperature is measured by the NHQM103B375T10 thermistor designed by Thermometrics. These thermistors are much like the thermistors successfully designed and tested on $\mathrm{CP} 1$. The thermistor will change resistance with temperature and the temperature is converted to a voltage by using the thermistor in a voltage divider. In Figure 26, RT1 and RT2 are the thermistors and R14 and R15 are used with the thermistors to create an analog voltage that will then be sensed by the ADC.

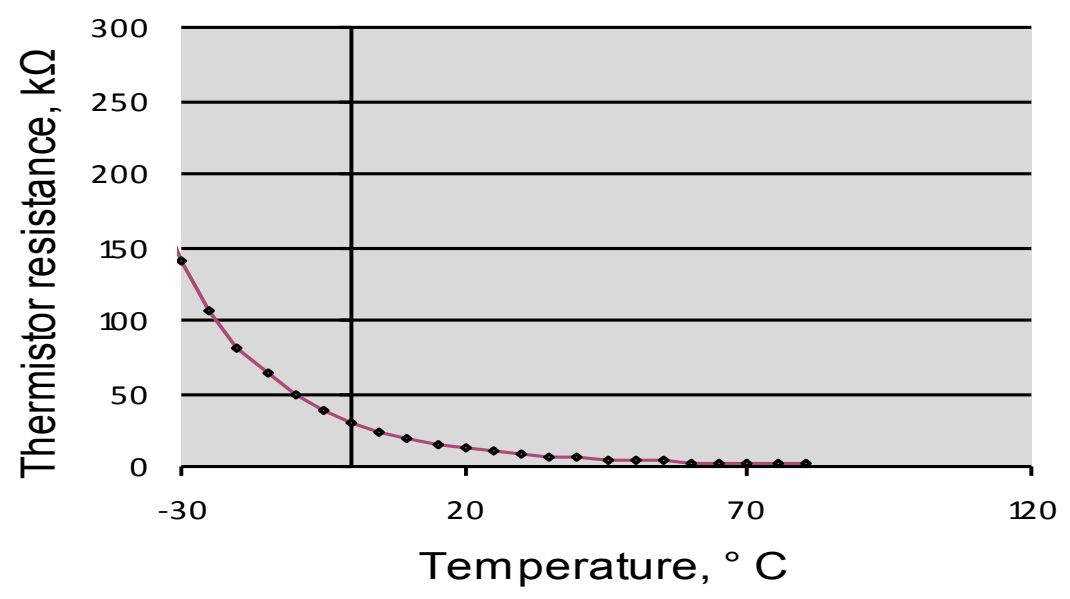


Figure 129. Resistance to Temperature for a NHQM Series Thermistor by Thermometrics. 9

The thermistor resistance is a log function of the temperature. This makes the voltage out of the voltage divider highly non-linear. The resistance to temperature curve is shown in Figure 27. The ADC has an analog input voltage range from zero to two volts. With the ADC input voltage range and resolution known, a graph ADC code to temperature has been created. This graph is shown in figure 130.

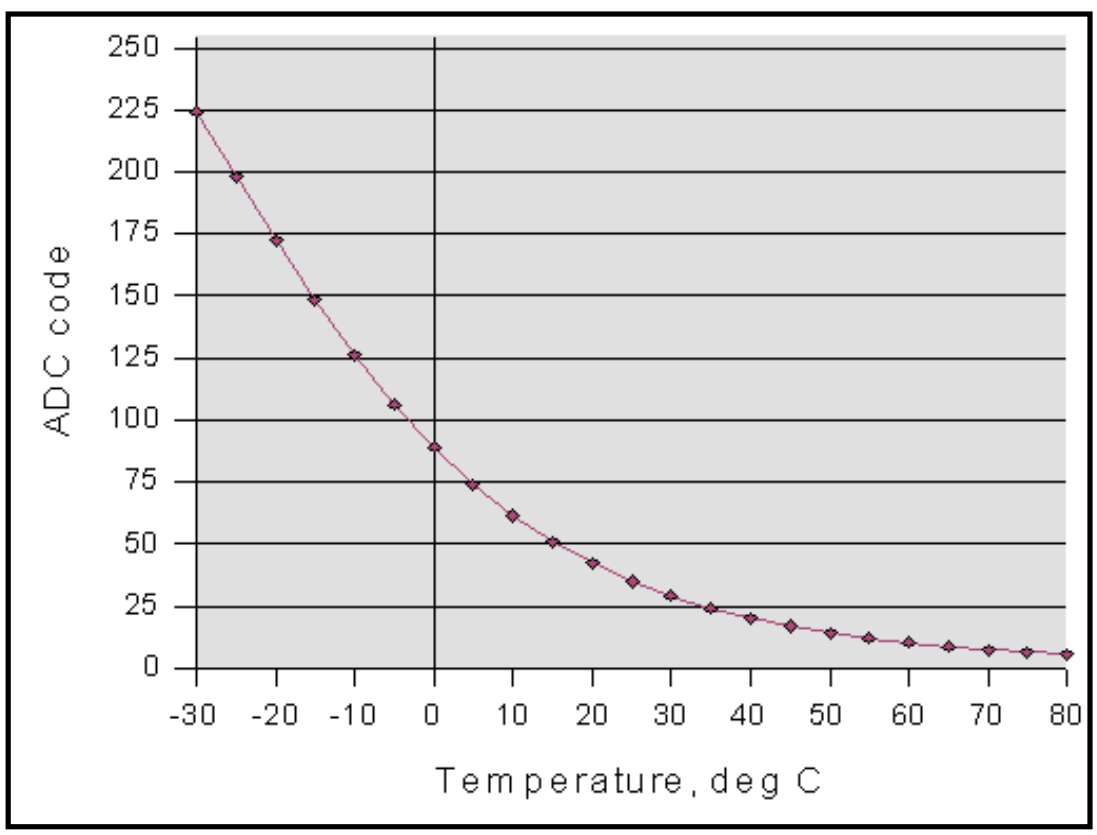

Figure 130. ADC Code vs. Thermistor Temperature.

The values of the $10 \mathrm{k} \Omega$ thermistor and the $100 \mathrm{k} \Omega$ resistor in series with it have both been chosen for a few reasons. First, the $100 \mathrm{k} \Omega$ resistor has been chosen because it prevents the maximum current consumption through the temperature sensing circuit to be a maximum of $30 \mathrm{uA}$. The actual current consumption varies 
with the thermistor's resistance. Resistances considerably higher than $100 \mathrm{k} \Omega$ cause the input leakage current of the ADC to affect the accuracy of the temperature measurement.

The $10 \mathrm{k} \Omega$ thermistor's value is selected because it gives an appropriate range of temperature over which the ADC will receive an appropriate voltage range. The thermistor voltage divider circuit is supplied with $3 \mathrm{~V}$ from the regulated side panel $3 \mathrm{~V}$ line. Since the thermistor will vary the voltage divider's output voltage and the $\mathrm{ADC}$ has an input voltage range of $0-2 \mathrm{~V}$, the thermistor resistance must not cause the $A D C$ 's input voltage to reach above $2 \mathrm{~V}$ over the operating temperature range of the satellite. In order to keep the resistor divider voltage in the correct range, the $10 \mathrm{k} \Omega$ thermistor is selected. This thermistor resistance value give a voltage in the range that the $A D C$ can read between temperatures of $-35^{\circ} \mathrm{C}$ and $80^{\circ} \mathrm{C}$. The $10 \mathrm{k} \Omega$ thermistor's resistance value means that its resistance is around $10 \mathrm{k} \Omega$ at $25^{\circ} \mathrm{C}$.

From Figure 28, it is clear that the ADC codes change less frequently at higher temperatures than at low temperatures. It is desirable for ADC codes to change frequently as it gives better resolution. For example, if the temperature changed by $0.5^{\circ} \mathrm{C}$ per $\mathrm{ADC}$ code, this would provide a one half degree temperature sensing resolution. While the temperature sensing resolution is very good or adequate over most of the operating temperature range, the number of degrees per ADC code increases significantly near the upper end of the operating temperature range. For example, at $60^{\circ} \mathrm{C}$, the temperature sensing resolution is 
$3^{\circ} \mathrm{C}$ per $\mathrm{ADC}$ code. While this is a widely spaced temperature resolution, we do not need a very precise resolution and this circuit meets our requirements.

The Thevenin equivalent resistance of the thermistor voltage divider as seen from the ADC varies widely and can affect the accuracy of the ADC reading. The ADC's input leakage current can reach +-1uA and the resistance of the voltage divider is between $58 \mathrm{k} \Omega$ and $1.4 \mathrm{k} \Omega$. This range gives a worst case error of about 7 ADC codes at -30 degC and 4 ADC codes or less at -10 degC and higher temperatures.

Some other methods of converting temperature to an analog voltage are with a temperature sensing IC such as the LM20 series or with a thermocouple and thermocouple interface using an IC such as the AD594. 


\section{APPENDIX P. GeneSat-1 Telemetry and Temperature Ranges}

Some CubeSats, such as NASA's GENESAT shown below in figure 131, must

have a thermal control system in order to complete their mission. GENESAT was carrying live bacteria that would quickly perish over one orbital revolution in the normal environment of a CubeSat so a small strip heater was integrated with their payload system. As a triple cube they had the power and mass available and as a NASA mission their budget also provided for the effort required to integrate a closed loop heater system.

Even with an active heater element, $4 \mathrm{~kg}$ for thermal inertia and an attractive 410 km circular orbit the GeneSat bus sees constant thermal cycling.

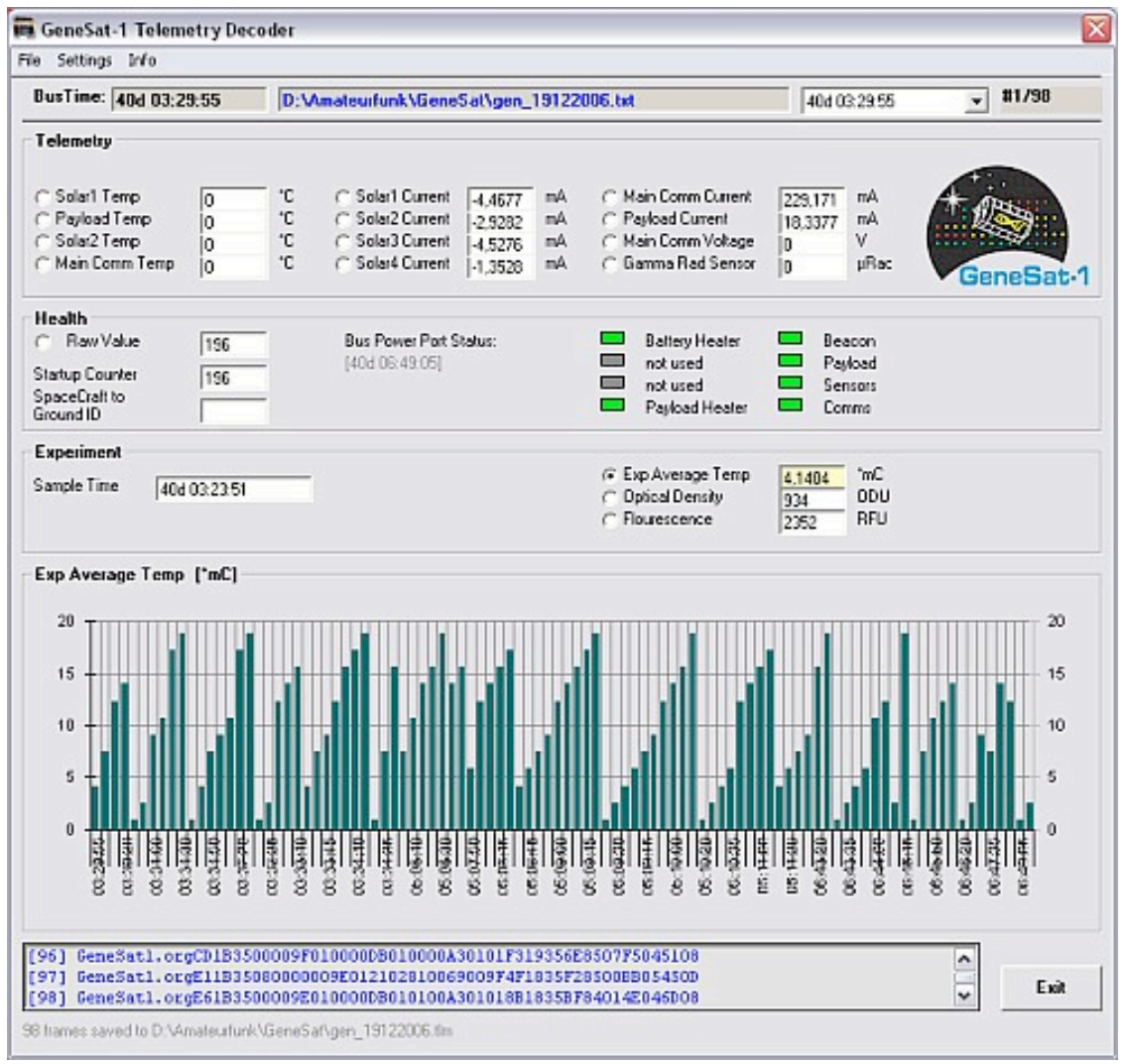

Figure 131. GeneSat-1 Temperature History (External) ${ }^{38}$ 


\begin{tabular}{|c|c|c|c|c|c|c|c|c|c|}
\hline \multicolumn{10}{|c|}{ Beacon Data } \\
\hline Name & Description & Type & $\begin{array}{l}\text { Plot } \\
\text { Units }\end{array}$ & Sensor Location & $\begin{array}{l}\text { Curve } \\
\text { Type } \\
\end{array}$ & Formula & m & b & Comments \\
\hline BusTime & System Time & Time & Sec & C and DH Eoard & Abscissa & $\begin{array}{l}\text { TimeStamp=INTEGE } \\
\text { R(CT) }\end{array}$ & $\mathrm{na}$ & na & 4 bytes. Counts in seconds. \\
\hline Tempt & $\begin{array}{l}\text { Temp @ Solar } \\
\text { Panel } 11\end{array}$ & $\begin{array}{l}\text { AD590, SN: } \\
14\end{array}$ & Deg-C & $\begin{array}{l}\text { Center, Mounting } \\
\text { Panel }\end{array}$ & Ine & $\mathrm{T} 1=\mathrm{m}^{n}(\mathrm{CT})+\mathrm{b}$ & 0.0453 & -1.107 & $\begin{array}{l}\text { X range }=0-1023 \text {, Temp. range }=-1.11 \mathrm{C}- \\
45.27 \mathrm{C}-\end{array}$ \\
\hline Temp2 & $\begin{array}{l}\text { Temp a } \\
\text { Payload Enclose }\end{array}$ & ${ }_{18}^{\text {AD590, SN: }}$ & Deg-C & $\begin{array}{l}\text { Center, Optic side, } \\
\text { outside enclose }\end{array}$ & Ine & $\mathrm{T} 2=\mathrm{m}^{2}(\mathrm{CT})+\mathrm{b}$ & 0.0456 & -1.299 & $\begin{array}{l}\text { Xrange }=0-1023 \text {, Temp. range }=-1.3 \mathrm{C}- \\
4538 \mathrm{C}\end{array}$ \\
\hline Temp3 & $\begin{array}{l}\text { Tenp } 9 \text { Solar } \\
\text { Panel } 2\end{array}$ & $\frac{\text { ADE90, SIN: }}{16}$ & Deg-c & $\begin{array}{l}\text { Center, Mounting } \\
\text { Panel }\end{array}$ & Finte & $T 5=m^{*}(C T)+b$ & 0.0458 & -0.6926 & $\begin{array}{l}\text { Xrange }=0-1023 \text {, Temp. range }=-0.69 \mathrm{C}- \\
46.15 \mathrm{C}\end{array}$ \\
\hline Temp4 & $\begin{array}{l}\text { Temp @ Main } \\
\text { Comm }\end{array}$ & $\begin{array}{l}\text { AD590, SN: } \\
20\end{array}$ & Deg-C & $\begin{array}{l}\text { Side close to the } \\
\text { anterna }\end{array}$ & Ine & $T 6=m^{*}(C T)+b$ & 0.0452 & -1.1886 & $\begin{array}{l}\text { X range }=0-1023 \text {, Temp. range }=-1.19 \mathrm{C}- \\
45.09 \mathrm{C}\end{array}$ \\
\hline Solar1l & $\begin{array}{l}\text { Solar Panel } 1 \\
\text { Current }\end{array}$ & Current & $\mathrm{mA}$ & EPS & line & Solarll $=m+(C T)+b$ & 0.9569 & -4.4677 & $\begin{array}{l}\text { Xrange }=5-1023 \text {, Currentrange }=0.33 \mathrm{~mA} \\
99774 \mathrm{~mA}\end{array}$ \\
\hline Solar21 & $\begin{array}{l}\text { Solar Panel } 2 \\
\text { Current }\end{array}$ & Current & $\mathrm{mA}$ & EPS & line & Solar1l $=m^{*}(C T)+b$ & 0.9581 & -2.9282 & 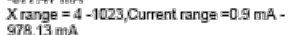 \\
\hline Solar31 & $\begin{array}{l}\text { Solar Panel } 3 \\
\text { Current }\end{array}$ & Current & $\mathrm{mA}$ & EPS & line & Solar $11=m^{*}(C T)+b$ & 1.0346 & -4.5276 & $\begin{array}{l}X \text { range }=2-1023 \text {, Currentrange }=0.55 \mathrm{~mA} \\
-976.4 \mathrm{~mA}\end{array}$ \\
\hline Solaral & $\begin{array}{l}\text { Solar Panel } 4 \\
\text { Current }\end{array}$ & Current & $\mathrm{mA}$ & EPS & line & Solar $\|=m "(C T)+b$ & 0.9556 & -1.3526 & $\begin{array}{l}\text { Xrange }=2-1023, \text { Current range }=0.56 \mathrm{~mA} \\
97737 \mathrm{~mA}\end{array}$ \\
\hline Comml & $\begin{array}{l}\text { Main Comm } \\
\text { Current }\end{array}$ & Current & $\mathrm{mA}^{\mathrm{A}}$ & EPS & line & Comml $=m r^{2}(C T)+b$ & 2.1126 & 7,3483 & $\begin{array}{l}X \text { range }=1-485 \text {, Current range }=9.46 \mathrm{~mA} \text { - } \\
1031.97 \mathrm{~mA} \text {, Circuit will be cut off if CT }>485 \\
\text { or l }>1031 \mathrm{~mA}\end{array}$ \\
\hline PLI & Payload Current & Current & $\mathrm{mA}$ & EPS & line & $\begin{array}{l}\text { Payload11 = } \\
\text { m. }^{2}(\mathrm{CT}) * \mathrm{~b}\end{array}$ & 0.4791 & 0.611 & $\begin{array}{l}\text { X range }=0.975 \text {, Current range }=0.511 \mathrm{~mA} \\
-457.7 \mathrm{~mA} \text { Circuit will be cut of if } \mathrm{CT}>975 \\
\text { or } 1>457.7 \mathrm{~mA} \text {. }\end{array}$ \\
\hline CommV & $\begin{array}{l}\text { Main Comm } \\
\text { Voittage }\end{array}$ & Voltage & Volts & EPS & line & $\mathrm{CommV}=m^{*}(\mathrm{CT})+\mathrm{b}$ & 0.012 & -0.012 & $\begin{array}{l}\text { On } X \text { range }=213-437 \text {, On Voltage range } \\
=5 \mathrm{~V}-2 . \mathrm{VV} \text {, Circult will be cut off if } \mathrm{CT}<213 \\
\text { of } \mathrm{V}<2.8 \mathrm{~V}\end{array}$ \\
\hline RadCount & $\begin{array}{l}\text { Gamma } \\
\text { Radiation } \\
\text { Sensor }\end{array}$ & Radiaton & uRad & $658 \mathrm{CT}$ average. & line & $m R a d=m^{*}(C T)+b$ & 0.0015 & 0 & 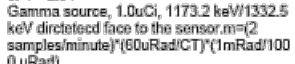 \\
\hline Health & $\begin{array}{l}\text { Subsystems } \\
\text { Power State }\end{array}$ & Boolean & N/A & па & $\mathrm{na}$ & na & & & 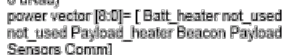 \\
\hline \multirow{4}{*}{$\begin{array}{l}\text { ExpSampl } \\
\text { eTime } \\
\text { ExpTemp } \\
\text { Mell } \\
\text { Well } \\
\text { Number } \\
\text { ExpoD }\end{array}$} & \multirow{4}{*}{$\begin{array}{l}\text { Experiment } \\
\text { Time } \\
\text { Exp.Average } \\
\text { Temp. } \\
\text { Weilt being } \\
\text { measured } \\
\text { Optical Density } \\
\text { Reading }\end{array}$} & Time & Sec & \multirow{4}{*}{$\begin{array}{l}\text { Payload Main } \\
\text { Electronins } \\
\text { Payload Heater } \\
\text { Plate } \\
\text { Software } \\
\text { incrementer } \\
\text { Optics, TAOS } \\
\text { Detector }\end{array}$} & Abscissa & \multirow{2}{*}{$\begin{array}{l}\text { TimeStamp= } \\
\text { INTEGER(CT) } \\
\text { TM=mt(CT)+b }\end{array}$} & na & па & \multirow[t]{2}{*}{$\begin{array}{l}\text { Sensors Commi] } \\
4 \text { Bytes. Ccunt's in seconds. }\end{array}$} \\
\hline & & \multirow{4}{*}{$\begin{array}{l}\text { Temperature, } \\
\text { mean } \\
\text { linteger } \\
\text { Enumerator } \\
\text { Freq as } \\
\text { Function of } \\
\text { light } \\
\text { Freq as } \\
\text { Function of } \\
\text { Eight }\end{array}$} & Degc & & Fine & & 0.006400 & 0.0124 & \\
\hline & & & Well \# & & & Well=INTEGER(CT) & $\mathrm{na}$ & na & \multirow{2}{*}{$\begin{array}{l}\text { Well number used to ID Darkl, OD, and FL } \\
\text { data below. Uses } 1 \text { byte in the data packet. } \\
\text { xio to remioue ontoard divide by } 10 \text {. }\end{array}$} \\
\hline & & & oDu & & Ine & OD_Taos $=m^{*}(C T)+b$ & 1 & 0 & \\
\hline ExpFL & $\begin{array}{l}\text { Flourescence } \\
\text { Reading }\end{array}$ & & RFU & $\begin{array}{l}\text { Optirs, TAOS } \\
\text { Detector }\end{array}$ & Ine & FL_T & 1 & 0 & $x 10$ to remove enboard diüde by 10. \\
\hline
\end{tabular}

Figure 132. GeneSat-1 Beacon Data Including Thermal Ranges ${ }^{38}$ 